RECURSIONS

$$
\begin{aligned}
& \text { Media } \\
& \text { Infrastruotures } \\
& \text { and the Politios } \\
& \text { of Digital Time }
\end{aligned}
$$

Essays on

Hardwired

Temporalities 
Media Infrastructures and

the Politics of Digital Time 
The book series RECURSIONS: THEORIES OF MEDIA, MATERIALITY, AND CULTURAL TECHNIQUES provides a platform for cuttingedge research in the field of media culture studies with a particular focus on the cultural impact of media technology and the materialities of communication. The series aims to be an internationally significant and exciting opening into emerging ideas in media theory ranging from media materialism and hardware-oriented studies to ecology, the post-human, the study of cultural techniques, and recent contributions to media archaeology. The series revolves around key themes:

- The material underpinning of media theory

- New advances in media archaeology and media philosophy

- Studies in cultural techniques

These themes resonate with some of the most interesting debates in international media studies, where non-representational thought, the technicity of knowledge formations and new materialities expressed through biological and technological developments are changing the vocabularies of cultural theory. The series is also interested in the mediatic conditions of such theoretical ideas and developing them as media theory.

\section{Editorial Board}

- Jussi Parikka (University of Southampton)

- Anna Tuschling (Ruhr-Universität Bochum)

- Geoffrey Winthrop-Young (University of British Columbia) 


\title{
Media Infrastructures and the Politics of Digital Time
}

Essays on Hardwired Temporalities

\author{
Edited by \\ Axel Volmar and Kyle Stine
}


Gefördert durch die Deutsche Forschungsgemeinschaft (DFG) - Projektnummer 262513311 - SFB 1187. Funded by the German Research Foundation (DFG) - Project-ID 262513311 - SFB 1187 .

Funded through a Social Sciences \& Humanities Research Council of Canada (SSHRC)

Connection Grant, file number 611-2015-0336.

Cover illustration: Pete Linforth (Pixabay), Pavlofox (Pixabay).

Cover idea: Julia Eckel

Cover design: Suzan Beijer

Lay-out: Crius Group, Hulshout

ISBN $\quad 9789463727426$

e-ISBN $\quad 9789048550753$

DOI $\quad 10.5117 / 9789463727426$

NUR $\quad 757 \mid 811$

\section{(c) $(1) \Theta \Theta$}

Creative Commons License CC BY NC ND

(http://creativecommons.org/licenses/by-nc-nd/3.o)

@ All authors / Amsterdam University Press B.V., Amsterdam 2021

Some rights reserved. Without limiting the rights under copyright reserved above, any part of this book may be reproduced, stored in or introduced into a retrieval system, or transmitted, in any form or by any means (electronic, mechanical, photocopying, recording or otherwise).

Every effort has been made to obtain permission to use all copyrighted illustrations reproduced in this book. Nonetheless, whosoever believes to have rights to this material is advised to contact the publisher. 


\section{Table of Contents}

Acknowledgments

Infrastructures of Time: An Introduction to Hardwired Temporalities

9

Kyle Stine and Axel Volmar

\section{Part I Media Philosophies of Time Patterning}

1. The Suspension of Irreversibility: The Fundamental (and Futile) Task of Media

John Durham Peters

2. Time and Technology: The Temporalities of Care

Gabriele Schabacher

3. Problems of Temporality in the Digital Epoch

Yuk Hui

4. Suspending the "Time Domain": Technological Tempor(e)alities of Media Infrastructures

Wolfgang Ernst

\section{Part II Microtimes}

5. Infrastructuring Leap Seconds: The Regime of Temporal

Plurality in Digitally Networked Media Isabell Otto

6. Life at the Femtosecond

Geoffrey C. Bowker

7. Artificial Intelligence and the Temporality of Machine Images Andrew R.Johnston

8. Intervals of Intervention: Micro-Decisions and the Temporal Autonomy of Self-Driving Cars 


\section{Part III Lifetimes}

9. Grounded Speed and the Soft Temporality of Network Infrastructure

Nicole Starosielski

10. Unruly Bodies of Code in Time

Marisa Leavitt Cohn

11. Screwed: Anxiety and the Digital Ends of Anticipation James J. Hodge

12. Beep: Listening to the Digital Watch

Sumanth Gopinath

\section{Part IV Futures}

13. Captured Time: Eye Tracking and the Attention Economy Alexander Monea

14. Ahead of Time: The Infrastructure of Amazon's Anticipatory Shipping Method 263 Eva-Maria Nyckel

15. Artificial Neural Networks, Postdigital Infrastructures and the Politics of Temporality Andreas Sudmann

16. Technics of Time: Values in Future Internet Development

Britt S. Paris

Index 


\section{Acknowledgments}

This collection stems from two conversations started at McGill University in Montréal, Canada, in 2015 and the University of Siegen, in Siegen, Germany, in 2018. We express our gratitude to the Social Sciences and Humanities Research Council of Canada (SSHRC), which generously funded the project through a Connection Grant, as well as the Andrew W. Mellon Foundation, the Dean of Arts Development Fund of the Faculty of Arts at McGill University, and McGill's Post-Graduate Students' Society (PGSS). We extend special thanks to Jonathan Sterne for his support. Additionally, we thank Errol Salamon, Maryse Ouellet, Anastasia Howe Bukowski, and Megan Mericle for logistics and organizing; Caitlin Loney for designing our website, posters, and flyers; and Kathleen Holden for administering the awards funds in the Faculty of Arts. We also owe thanks to the Collaborative Research Center "Media of Cooperation" at the University of Siegen, funded by the German Research Foundation (Deutsche Forschungsgemeinschaft, DFG), and to Jenny Berkholz and Anja Höse. Finally, we would like to extent special thanks to the German Research Foundation for making this volume freely available via Open Access. 



\title{
Infrastructures of Time: An Introduction to Hardwired Temporalities
}

\author{
Kyle Stine and Axel Volmar
}

\begin{abstract}
The introductory essay to the volume proposes a framework for understanding the transformative and disruptive effects of digital time. It argues for a multiscalar approach to the layers of temporality active in current media infrastructures, which coordinate different magnitudes of time from the microtemporal to the longue durée. Situating the phenomenon of digital time within a trajectory of increasing materialization of temporal relations, it provides a historical account of the becoming concrete in technology of what were once relations between people and objects.
\end{abstract}

Keywords: media theory, digital time, infrastructures, materiality, temporality

\begin{abstract}
All machines, whether mechanical, electronic, or symbolic, are in a crucial sense time machines. They pattern the movement of mechanisms, the flow of electrons, or the operations of symbols to meet temporal demands such as synchronism, succession, repetition, and pace. Media technologies thus constitute not only material infrastructures, as has been a watchword in recent media theory, but also temporal infrastructures, architectures, and systems - materialities designed in and as time. An aspect of this patterning of time that has received heightened scholarly attention is the ubiquitous experience of technological and cultural acceleration. Temporal speed-up has in fact emerged as a defining characteristic in accounts of modernity, as Peter Conrad expresses in saying, "Modernity is about the acceleration of time." Recent works, from critical theory to the sociology of time, have
\end{abstract}

Volmar, A. and K. Stine (eds.), Media Infrastructures and the Politics of Digital Time: Essays on Hardwired Temporalities. Amsterdam: Amsterdam University Press, 2021 DOI 10.5117/9789463727426_INTRO 
emphasized this in terms of a cultural doctrine of accelerationism. ${ }^{2}$ However, focusing on the temporal aspects of media culture reveals not only an acceleration of life and communication systems but also complex temporal relations within technologies, between technologies, and between human time and technological time.

Take a smartphone, for example. A host of services are at one's fingertips: rides available for pickup at an exact address, rooms ready to be reserved and digital keys accessed without interaction with any person, goods connected to whole systems of order delivery through automated warehouses and same-day transportation, and entertainment media set to stream on the go. Such ease of access can give the impression of instantaneity and immediacy, of time compressed to the zero degree. There are no lapses in programming, such as when television stations of old shut down for the night, and no closed signs to be flipped on shop doors. ${ }^{3}$ Yet obvious from experience is that these conveniences are also subject to service interruptions, scheduled maintenance, system lag, and downtime, not to mention rush hours and peak pricing. Behind and beneath our real-time interactions with on-demand media and services is a temporal geography as uneven as our social and political geographies, in which slowness and waiting are produced and distributed alongside every advance in convenience and speed.

Recent research in media and cultural studies has attended closely to the widening gap in lived experiences of time across different social geographies. Emily Keightley in this regard urges media scholars "to move beyond a one-dimensional characterization in which speed and immediacy monopolize accounts of how time is encountered and lived" and instead address the "social temporalities of mediated experience." Sarah Sharma shows in her ethnography of business travelers that the experience of living "fast," as promoted throughout self-help literature on time management, represents a luxury that draws on an entire service industry of workers made to calibrate their bodies to the demands of global capitalism. ${ }^{5}$ In the words of Markus Krajewski, modern service workers, or "servers," to express the comparison between people and technologies that are ordered to stand by, have always been consigned to "idle time," or waiting for a bell, command, or instruction that positions them in relation to the time they serve. ${ }^{6}$ In the essays collected in this volume, we follow these studies by understanding time in its unequal distribution across our social and political world, while emphasizing its particular relation to the problem of technological acceleration, the stark departure of technological time from lived human time, and in this way couple the themes of social inequality and accelerationism in the analysis of time. 
The scale of this inquiry cannot be addressed solely at the level of the individual medium or technology, in that the defining characteristic of digital time is that it coordinates multiple layers of technological time within a comprehensive system. The analytical figure of digital time is for this reason the infrastructure. On the one hand, infrastructures embody temporal relations between technologies, as maintained through standards and protocols; on the other hand, they coordinate relations between technologies and human beings, who serve as interlocutors, care givers, proxies, and delegates. So while digital technologies construct an uneven geography that accelerates life for some and impedes life for others, they also profoundly transform and reorder temporal regimes and practices in other ways that require critical attention.

A central argument of the volume is that the concerns of digital time and contemporary media infrastructures exceed any one field of inquiry, requiring cross-disciplinary perspective. Our objective is to account for devices and processes whose spheres of action range from the microtemporal to the geological, addressing for the time domain what studies have noted for the spatial domain of international logistics, whose vectors range from the flows of microscopic circuits to the paths of orbiting satellites. ${ }^{7}$ We pursue this goal through a broadly inclusive range of media and infrastructure studies, with perspectives from science and technology studies, cultural studies, and the philosophy of time, while acknowledging the need for future collaborations between the humanities, sciences, and engineering. The volume in this way serves as a meeting ground between disciplines and begins a cross-disciplinary conversation that will become increasingly relevant and necessary as infrastructural systems extend yet further into the social and political systems of everyday life.

The contributors study two aspects of the infrastructuring of time: the infrastructures of temporality, namely the means of ordering time through technologies and practices ranging from calendars to computers; and the temporalities of infrastructure, or the specific, often incompatible temporal orders of different technical milieu in media, science, business, and government. As our title suggests, the central metaphor of our inquiry, drawing on the predominant mode in which temporalities are inscribed and effectuated today, is the hardwiring and rewiring of temporal orders, calling attention to how stabilized temporalities, erected in infrastructures, exert pressures of conformity and standardization on the temporalities of lived experience and among different temporal infrastructures. Hardwired temporalities refers to all the ways that time patterns become fixed in materialities: these can occur through unplanned flows of habituation whose constancy eventually 
sculpts a definable space, just as flowing water carves out a river bed that endures even while remaining open to redirection; but they can also be formed by more obdurate temporal governance, the imposition of temporal patterns by top-down command. Temporal regimes refers to the guiding principles of ordering time in a given locality and epoch. The overriding temporal regime today is the imperative speedup of global capitalism, but smaller scale temporal orders exist within this larger regime, such as the orders of religious time and leisure time. Because networks so define social space, we speak of hardwiring and rewiring temporal relations, rather than using the more amorphous phrasing of temporal flows. Reorganization of temporal patterns in infrastructures is not liquid — it cannot take just any shape- but is nevertheless flexible and open to reconfiguration. Moreover, these patterns coordinate different spheres of action. The temporal orders of our digital culture involve infrastructural formations across multiple temporal scales, from the microtemporal domains of manipulating, processing, and transmitting information, through the temporal orders on the meso scale of everyday life and lived temporalities, to the macrotemporal scales of cosmological and geological deep time.

The collection speaks to and consolidates insights among three important directions in media studies today, making contributions in this way also to three broad pursuits in recent humanities and social science research. The current social and political unrest in the neoliberal economies, following an intensified concentration of wealth enabled by disruptive new technologies, has prompted a turn toward material culture and a deeper consideration of the technical specificities of the networks, devices, and programs used in our daily lives. This turn toward the nonhuman and emphasis on new materialisms for rethinking the relationship between human societies and technological networks has significantly broadened the scope of inquiry and deepened the scales of time considered. ${ }^{8}$ However, it also risks a certain spatializing bent. Jane Bennett points to one aspect of this bias when she notes the tendency in object-oriented ontology to disregard the relations between objects, in response to which she suggests a way of thinking objects and their relations together. ${ }^{9}$ We argue that time is precisely the dimension that is lost in an approach to objects that brackets out relations, and it is necessary to develop approaches to materiality that analyze time, which is fundamentally relational.

Our inquiry comes at the question of digital temporality from two directions. The first of these we have discussed in addressing the social experience of time and its multiplicity, what we can characterize, for the sake of comparison, as a culturally focused approach. However, we follow the insights of science and technology studies to understand that no technical 
system or device is value neutral. Any divergence between the temporal patterns of human life and those of networked technologies is likely to exacerbate inequalities already perpetuated by systemic discrimination. The last two years have seen important interventions in the areas of technological and algorithmic bias, whose insights point to ways scholars might further interrogate the uneven distribution of time. The attention economy's effort to maximize engagement is an explicit program to monopolize people's time. ${ }^{10}$ When biased algorithms filter results in discriminatory ways, as Safiya Umoja Noble has shown, they not only misrepresent people and concepts; they also misdirect people and consume their time. ${ }^{11}$ Charlton McIlwain and Ruha Benjamin have pointed out how technological systems that present as neutral means of problem-solving are cut through with racial biases. Early computer systems, as McIlwain demonstrates, were explicitly intended to aid police countermeasures against the Civil Rights Movement and were further embedded in the carceral apparatus of the War on Drugs. ${ }^{12}$ Benjamin has extended this insight in the deepest way to show that, even beyond the point-of-the-sword biases of facial-recognition software and search tools that predict ethnicity according to people's names, technological systems entrench racial hierarchies throughout their design in myriad ways that are inescapable in their effects. ${ }^{13}$ The continual march of innovation, which hardwires and rewires power relations, deserves further attention in analyses of time, a framework to which the essays in this volume seek to contribute.

Complementary to the perspective on lived time is an approach that draws insights from German media theory, ${ }^{14}$ media archaeology, ${ }^{15}$ and studies of microtemporalities, ${ }^{16}$ with their attention to the design and inner workings of the technologies themselves, to extend an analysis of time beyond representational media, such as literature and film, ${ }^{17}$ to the nonrepresentational media and programs that enable them, often invisibly. Following Wolfgang Ernst, we understand that media studies must become "time-critical."18 Being sensitive to the time-criticality of media technologies means being attentive to temporal actions that are in a certain way "critical factors" for the successful execution of a process. ${ }^{19}$ This includes real-time applications, whose operations exist below the threshold of human perception, and more generally the synchronization and coordination of different co-operative speeds and time windows. The volume is thus informed by and speaks to the current German discourse on understanding media less as means of representation and transmission than as fundamental "conditions of cooperation," a conversation closely connected to the "practice turn" in media theory. ${ }^{20}$

Lastly, the volume benefits from and contributes to the growing interest in infrastructures. ${ }^{21}$ We take our cue from Susan Leigh Star and Geoffrey 
Bowker's notion of "infrastructuring" as an active, ongoing process and from Lauren Berlant's recent broadening and refinement of the concept of infrastructure to mean "the movement or patterning of social form." ${ }^{22}$ Berlant's choice of the word "patterning," in its active, gerundial sense, as opposed to the more static connotations of "pattern" and "form," lays emphasis on infrastructures as temporal processes of becoming. Social patterns, however fixed they may seem, are only ever the circuit for movements and temporal flows. Keller Easterling has shown how infrastructure space, even when not "mediated" in the common sense by sensors and media technologies, is an information technology where the mere mobilization of form, in grids and containers, is "an operating system for shaping the city."23 We might argue, in a similar way, that infrastructure time, the rhythm and patterning of temporal order, is the very basis of information, which never stands still but must be processed and transmitted. Indeed, recent developments in network technologies and smart sensors, we argue, have created a need to reexamine infrastructures particularly in terms of their patterning of time. "To be modern," as Paul Edwards puts it, "is to live within and by means of infrastructures." ${ }^{24}$ To be digital—or to be in an algorithmic, networked culture - is to live within and by means of infrastructures that are themselves monitored, maintained, and controlled by deeper data infrastructures. The infrastructures of modernity, such as roads, bridges, communication lines, and financial systems, have long been equipped with cybernetic feedback infrastructures that monitor their operations, make corrections, and, when needed, marshal workers to repair them. ${ }^{25}$ The temporalities of their operation and aging are now bound up in the computational time of digital networks and, as such, submitted to the control and surveillance of these networks. Yet at the same time, these newer infrastructures rely on the older infrastructures of water, energy, and human transit, as a nervous system relies on a circulatory system. Infrastructures in this way are coordinating and synchronizing features of multiscalar action, which, as we have mentioned, embody relations between technologies and people, and are thus appropriate figures for thinking about materiality, microtemporalities, and the social geography of time together.

\section{Technologies as Consolidated Temporalities}

A guiding thread in this regard involves the processes by which technical systems consolidate temporality, in the most literal sense of their gathering together disparate temporal processes and making them solid in physical 
infrastructures. Scholars of the social construction of science and technology teach us that even nonrepresentational technologies, which cultural analysis long overlooked in favor of media and artistic works, embody social values and relations of power. In Bruno Latour's apt phrasing, technologies are "full of people": they concretize human expertise and function to advance human goals. ${ }^{26}$ Just the same, technologies are full of time. Karl Marx described one aspect of this when he argued that the value of the commodity consists in "congealed labor-time." ${ }^{27}$ Yet even beyond the actions necessary for their immediate manufacture, technologies embed multiple histories and spheres of temporal action. Michel Serres calls technologies "polychronic" to express the multiple pleats of time that fold together "the obsolete, the contemporary, and the futuristic":

Consider a late-model car. It is a disparate aggregate of scientific and technical solutions dating from different periods. One can date it component by component: this part was invented at the turn of the century, another, ten years ago, and Carnot's cycle is almost two hundred years old. Not to mention that the wheel dates back to neolithic times. The ensemble is only contemporary by assemblage, by its design, its finish, sometimes only by the slickness of the advertising surrounding it. ${ }^{28}$

Comprising parts of different vintage, technologies also operate on multiple scales of temporality. To use Serres's example, a modern car going at 60 miles per hour is likely to have wheels rotating at around 800 revolutions per minute, an engine firing at 2,000 revolutions per minute, and a microprocessor calculating at $2 \mathrm{GHz}$, or 120 billion cycles per minute. Meanwhile, the driver must maintain a safe reaction time, at best 0.7 seconds, and endure the length of travel, sometimes numbering several hours, interrupted by cyclical human events such as stopping off for food or at a rest area. Human-scale actions and technological actions, as this example suggests, are divergent along many paths, guided by their own temporal logics, but they are importantly coordinated and synchronized by means of cultural techniques of time patterning. ${ }^{29}$ The frame for thinking this coordination must come from temporal infrastructures, which by design integrate and mediate these various human and nonhuman temporalities, drawing together the micro, meso, and macro domains, and thereby enable systematization and diverse forms of cooperation..$^{30}$

Not only do temporal practices and processes tend to consolidate into temporal infrastructures; they also reciprocally interact as hardwired infrastructures with new kinds of pliable and adaptable systems, both 
above and below: above, in the flexible software systems designed to operate using new circuit generations and the various social practices centered on new communication technologies; and below, in the flexible economies and manufacturing processes at the base of fabricating these technologies. This dialectic between rigidness and flexibility, structure and versatility, predictability and unpredictability, as Serres notes, lies at the very heart of temporality. It is for this reason that the Latin tempus gives us not only the structured "temporality" of the clock but also the intemperate fluctuations of "temperature," the wild swings of "temperament," and the fierce unpredictability of "tempests":

The French language in its wisdom uses the same word for weather and time, le temps. At a profound level they are the same thing. Meteorological weather, predictable and unpredictable, will no doubt someday be explainable by complicated notions of fluctuations, strange attractors. Someday we will perhaps understand that historical time is even more complicated..$^{11}$

John Durham Peters elaborates these etymological connections in broad perspective:

In Latin, tempus means weather and time, giving English such words as temporal and tempest, and French le temps and Spanish el tiempo, both of which mean both time and weather; the Spanish al tiempo means both "in season" (of fruits) or "at room temperature" (of drinks). Terms such as temperature, tempering, tempo, and temperament show shared semantic fields across heat, harmony, rhythm, and mood. ${ }^{32}$

Time, in this sense, is moody and multiplicitous, varied and in flux. The goal of infrastructuring time, founded on time technologies and cultural techniques of time management, is to tame these moody fluctuations and to submit them to ordering - to hardwire them into lasting temporal regimes or cultures.

By the same token, the term "hardwired" relates equally to embedded systems and more flexible practices involved in rewiring temporal orders. To use an example, computer components in the 196os, whose electrical layout would soon be characterized as hardwired, were at the time compared favorably "soft" against the fixed-program analyzers of yesteryear. The first recorded instance of the then-hyphenated "hard-wired" documents this usage, when a contributor to the journal Nuclear Instruments and Methods 
in 1965 , comparing the two generations of computers, wrote: "Another trend is the use of small computers instead of hard-wired analyzers."33 In this way, the conceptual ground of hardwiring is, in a deconstructive turn, precisely the opposite of being fixed and immutable. Technical consolidation is instead the product of a new kind of adaptive industry, a flexible economy founded on the production of a new component, the integrated circuit, capable of being hardwired and rewired across product generations. Moore's Law, formulated in the same year of 1965 to describe the regular doubling of circuit complexity from one fixed pattern to another, has since turned into a self-fulfilling prophecy, a stable temporality that serves to predict future technological progress. In a system of planned obsolescence, hardwired components come to enable the periodic "rewiring" of the systems they run. ${ }^{34}$

The hardwiring of space, as seen in the doubling of component density on microchips, thus structures time in new ways as well, setting industry on a regular course of introducing new product generations and creating a new density of temporal intervals, or actions that can occur in a given period of time, with increased processing speeds. It would not be a stretch to say that these computational components and their infrastructuralization into larger networks invented a new sphere of time in the same way that James Carey saw the telegraph as instituting a new regime of time a century before. In its ability to send messages faster than physical commodities, according to Carey, the telegraph rendered obsolete the system of arbitrage-the practice of buying low in one market and selling high in another-and redirected financial speculation into commodity futures. "In a certain sense," Carey writes, "the telegraph invented the future as a new zone of uncertainty and a new region of practical action." 35 That is, the telegraph initiated a new domain of time by hard wires. Judy Wajcman stresses the continued material pressures of such wires with the example of a recently laid fiber-optic cable between Chicago and New York: "While previous cables between the two cities had been laid along railway lines, the new cable takes the shortest route possible, even drilling through the Allegheny Mountains. It shaves 1.3 milliseconds off the transmission time of the earlier cables." ${ }^{36}$ Likewise, contemporary developments in big data, artificial intelligence, and machine learning can be regarded as a result of the "wires" that have gone into the microprocessors and networked environments that enable algorithms to operate over global information networks within ever smaller temporal intervals. ${ }^{37}$

Historically, temporal practices have tended to consolidate into technical objects and from objects into structures and infrastructures. The more organized the materialities, the more structured the temporalities. 
Our project thus seeks to reassess material infrastructures as stabilized structured temporalities, forms of patterned time sustained over given periods of history in formations of technologies, practices, and conventions that affect people's actions and experiences and are themselves subject to constant "rewiring." The two terms that organize our thinking on the topic, "hardwired" and "temporalities," in this way name a dynamic interaction between infrastructured temporalities and their continual interactions with more pliable, flexible, mortal, human systems.

\section{A Chronology}

Temporality, as seen throughout history but especially in an age of global digital networks, is palimpsestic. Concretized within temporal infrastructures and embedded in our experiences of temporality are multiple historical regimes successively layered and combined. A modern smartphone, for instance, which is subject to the timing of a processor clock and various network synchronization protocols below the level of human awareness, also remediates earlier temporal interfaces through apps such as calendars, clocks, and stopwatches. To take but one example, Chinese, Hebrew, and Islamic calendars are standard options on smartphones alongside the western Gregorian calendar, while apps are available for Persian and Tibetan calendars, among others, and even more remote historical calendars such as the Maya calendar. Indeed, the applications of timekeeping in new media are practically limitless. While not dismissing this multitude of applications and their complex interactions, it is possible to outline four overarching temporal regimes, or historical hegemonic temporal logics, that inhere in modern technologies and continue to structure temporal techniques and experiences, namely, calendar time, clock time, capitalist time, and technological microtime.

We understand these temporal logics to have emerged from multiple locally installed and trans-locally networked temporal orders rather than from any single authoritative center. After all, even a regime as centrally administered as medieval Christian time was not commanded by a single source of timekeeping but was instead made possible by a multitude of individual clocks and clock towers in every Christian settlement, involving procedures for displaying the time, as for instance by the chiming of bells, and people tasked with caring for the clocks and regularly setting the time. Likewise, the concept of capitalist time, which introduced the virtue of optimization, emerged from a proliferation of town bells and factory time 
clocks, not from a single timekeeper, and in this way resulted in ever new "rewirings" of procedures of production, organization, and cooperation.

An obvious way of understanding these four temporal orders, which historically succeed one another, is in their progressive refinement of counting time. Each method of measuring time, which is also characteristic of a historical epoch, sharpens the unit of measurement, while the epochs themselves shorten. Calendar time, incipient with the invention of writing, patterns longer intervals such as days and years, while Christian clock time, beginning in the twelfth century, enables the standardization of human practices within the span of a day through the divisions of hours. Beginning in modernity, capitalist time, especially gaining momentum in the nineteenth century, with its inherent logics of efficiency and acceleration, increasingly focuses on the shorter measures of minutes and seconds, building upon which technological microtime more finely divides temporal measure below human sense thresholds. Temporal units and the span of innovation in this way historically tighten. However, the command of time, always directed toward the future, has progressively expanded, as evidenced by contemporary megaprojects, including the decade-long construction of CERN's Large Hadron Collider and the nearly two-decade construction of the Three Gorges Dam in China, which beyond taking a long time to build, consumed billions of dollars of labor time, relying on various specialized workforces. ${ }^{38} \mathrm{In}$ a similar fashion, the historical record available in each temporal regime has also expanded, evidenced nowhere more conspicuously than in the introduction of deep geological time in the eighteenth century, but also apparent in the heightened resolution of historical data. In this sense, it is not enough to consider only the units of temporal control and the length of future time under the command of the present; we must also acknowledge the new scales of temporal complexity within shorter intervals. Just as a computer performs more actions in a second than is possible using conscious calculation, a megaproject facilitates and coordinates more actions in the span of a week or year than was possible in previous projects in previous eras. Put simply, technological time today is denser and more vivid than past times; it contains more action moments and has a much higher resolution. Within these denser frames of planning and action must be coordinated the many temporal measures of the actors involved, whether human or nonhuman, which operate across these four temporalities.

In what follows, we attempt to delaminate these various layers of temporal governance to better understand their historical sources and how they interact, combine, over-pattern, and stabilize in durable infrastructures. 


\section{Calendar Time}

Since the early beginnings of so-called "civilization," the life of the vast majority of people has been and still is governed not only by the natural temporal rhythms of seasons and cycles of day and night but also by temporal regimes, i.e., orders of patterned time sustained by technologies and practices of timekeeping and temporal organization. John Durham Peters, for instance, reminds us of the fundamental significance of the calendar as a cultural technique of social order and governance that, through the science of astronomy and the politics of calendar making, provided a means of predicting and determining recurring events, from yearly floods, as in Mesopotamia and Egypt, to holy days. ${ }^{39}$ From their earliest uses to the present day, calendars have served to track the succession of days by dividing the year into arbitrary intervals of months and weeks. Their temporal divisions allow for repetition and ritual and hence the coordination of social, economic, and religious life into structured temporal schemes, both past and future. Alongside the political and military control of space, or territory, as Harold Innis argued in Empire and Communications, the cultural control of time based on common cosmological, religious, or philosophical narratives and materialized into different time media has played an equally important role in securing the endurance of cultural-political entities. ${ }^{40}$

A direct line extends from our present computational timekeeping technologies back to the calendars of earlier empires. In an influential essay on time and human language, Émile Benveniste explains that the calendar owes its existence to a baseline computation. ${ }^{41}$ Paul Ricoeur explains Benveniste's insight especially clearly: "the features common to every calendar 'proceed' from the determination of the zero point of some computation." ${ }^{42}$ In this sense, the calendar can be viewed as an early form of computing whose logistical functions issue from three basic conditions: the establishment of an axial moment, e.g., in the common era of occidental civilization marked by the birth of Christ; the determination of whether an event occurred before or after the axis; and the measurement of intervals, such as days, weeks, months, and years. The calendar, as Ricoeur puts it elegantly, thus "cosmologizes lived time and humanizes cosmic time"43; it is the first technique to organize these different spheres of temporality and serve as a bridge between them.

Calendars, as tools of temporal social organization, are the first techniques to introduce what Benveniste calls "chronic time," a term he uses to encapsulate both calendar and clock time for their ability to join together interior subjective duration and exterior physical time within a coordinating 
grid that locates personal experience within cosmic rhythms. ${ }^{44}$ Out of this logistical construction arises a seeming paradox in that chronic time, which Benveniste says is the only time we generally encounter in our dayto-day lives, does not move; it is instead arrested and, to repeat Hamlet's lament, "out of joint" with our inner experience of time, which constantly slips away: "It might thus seem natural that the structure of chronic time should be characterized by permanence and fixity. Yet, at the same time, it must be realized that these characteristics result from the fact that the temporal organization of chronic time is actually intemporal. This is not a paradox. ${ }^{245}$ Chronic time is a rigid atemporality, and only for this reason can it situate passing events in relation to one another. To use the language of our title, chronic time is the hardwired a priori of our more flexible everyday experiences of and interactions with irreversible time. An insight that we can draw from Benveniste is that just as the calendar presents a grid of temporal reference for calculating events, today's computer systems and infrastructures extend this intemporal grid to new levels of complexity and acceleration and thereby enable not only new modes of calculative governance but also new variabilities in lived temporality.

\section{Clock Time}

In his foundational work in the history of technology, Lewis Mumford (1934) demonstrates how the unified time of monastic life in the Middle Ages precipitated the development of the mechanical clock and influenced the subsequent temporal coordination of people and technologies that enabled both the Scientific and Industrial Revolutions, a development that leads him to argue: "The clock, not the steam-engine, is the key-machine of the modern industrial age." ${ }^{\prime 6}$ Alongside the calendar, which forges cultural unity on the basis of common holidays and other social events throughout the year, the clock enables within this initial computation of calendar time more fine-grained coordinations of human activities and forms of cooperation throughout the day and week, particularly the organization and control of human labor.

Historically, the prime points of time reference were not calculated abstractions but physical, often cyclical, work-based particularities. The rising and setting of the sun marked the passage of days, the wilting of flowers and spoiling of foods influenced cycles of work and gathering, the passage of winters measured age, and the recurrence of ten moons promised that an expecting mother would soon give birth. Noting among other regularities of human life, such as "the beating of the pulse" and "the 
breathing of the lungs," Mumford cites practices of agrarian subsistence: "The shepherd measures from the time the ewes lambed; the farmer measures back to the day of sowing or forward to the harvest." ${ }^{27}$ James Henry Breasted, writing in 1935, noted how the lives of his contemporaries gained temporal meaning by reference to seasonal fluctuations: "Among certain Swedish peasants even at the present day a birthday may fall at the 'rye harvest' or at the "potato harvest." ${ }^{\prime 4}$ From similar examples of celestial and earthly timekeeping, Peters (2015) has argued that the movements of the skies and earth themselves constitute "elemental media." ${ }^{\text {"9 }}$

It would be a mistake, however, to suggest that the onset of regimented clock time did away with these corporeal and more sensible temporal measures. In his commanding work on the history of timekeeping, Eviatar Zerubavel cites a striking example of time formulation without recourse to calendar or clock from the opening of Kurt Vonnegut's Cat's Cradle: "When I was a younger man - two wives ago, 250000 cigarettes ago, 3000 quarts of booze ago." $5^{0}$ Drawing on more functional examples, he reminds us that even today it continues to be more appropriate "to designate the life expectancy of tires and running shoes in terms of mileage — or that of children's beds in terms of the child's weight - than in terms of years of use. ${ }^{.51}$ What has changed though is that these time references take on new meaning in an era when, as Mumford argues, "The modern industrial regime could do without coal and iron and steam easier than it could do without the clock." ${ }^{2}$ For, now, all of these more variable temporal measures are caught within the mesh of modern clock-based time.

The wresting of time away from personalized reference points has been crucial in establishing intersubjective social realities. Time-counting devices, from water clocks to later mechanical clocks, in situating individual actions within a common social frame, have allowed for the organization of work, the establishment of cultural identity through repetition, and the incorporation of these identities and processes into larger cultural configurations such as corporations and nations. The monastery, according to Mumford, was the first instrument for calculating this form of social time:

Within the walls of the monastery was sanctuary: under the rule of the order surprise and doubt and caprice and irregularity were put at bay. Opposed to the erratic fluctuations and pulsations of the worldly life was the iron discipline of the rule. Benedict added a seventh period to the devotions of the day, and in the seventh century, by a bull of Pope Sabinianus, it was decreed that the bells of the monastery be rung seven times in the twenty-four hours. These punctuation marks in the day were 
known as the canonical hours, and some means of keeping count of them and ensuring their regular repetition became necessary. ${ }^{53}$

In a similar way, modern technical systems and infrastructures should be thought of as instruments for calculating and managing time. As profound as was the clock's impact on social organization was its effect on mechanical processes. For Mumford, the clock set "the regular collective beat and rhythm" of a technical system by wedding together these regular mechanical actions with the synchronized movements of people. More than a mere counting device, it was also "a new kind of power-machine, in which the source of power and the transmission were of such a nature as to ensure the even flow of energy throughout the works and to make possible regular production and a standardized product."54 Timekeeping was, then, from the beginning, a means of not only coordinating human actions but also regulating and operating machinery. The clock's qualities of standardization, automatic action, precise gearing, accuracy, and reversibility allowed it to divide time and conquer it. Even more significant, by taking on these characteristics of space, time could be added, saved, and controlled, laying the conditions for capitalist time.

\section{Capitalist Time}

Until recently, the temporal orders of calendar and clock have been subject to a politics of time, struggles within societies based on conflicting interests between the state, economic and religious actors, and individuals over matters such as the recognition of holy days and the designation of work times. The convergence of meanings on May 1 in cultures of the northern hemisphere helps to illustrate these conflicting politics of time. First celebrated in response to the astronomical event of spring, the day was a seasonal festival of the return of the warm season. After being adopted by the international workers movement to commemorate the Chicago Haymarket massacre in the late-nineteenth century, the day became a further palimpsest when, during the First Red Scare in the early 1920s, it became a reactionary, unofficial holiday dubbed "Americanization Day" that the US Congress would later inscribe into law as Loyalty Day during the Second Red Scare in the 1950s. Concerns about the temporal politics of paid labor continue unabated today in negotiations over how many hours per day and per week employees should work, how much vacation time should be allowed, how many sick days employers and health insurers should pay for, how overtime should be compensated, and how the post-work life of 
retirement should be managed. These struggles are largely crystallized in agreements, contracts, laws, and other forms that set more or less specific conventions for the patterning of human everyday life. 55

In this regard, E. P. Thompson argued that the decisive change paving the way for modern capitalism was the shift from task-oriented labor to time-oriented labor. ${ }^{6}$ Labor focused on tasks such as fishing and harvesting crops is embedded in the rhythms of the natural world, such as the rising and falling of tides and the passage of the seasons, and thus ritually connected to universal time. Such labor, rather than being set to the employer time clock, is characterized by "alternate bouts of intense labor and of idleness."57 The continuation of this labor pattern in the creative economy today leads one to wonder, as Thompson himself wondered in the 196os, whether "it is not a 'natural' human work-rhythm. ${ }^{8} 8$ But possessing one's natural time is largely at odds with capitalist economics. By uprooting time from one's personal experience, it becomes abstract and controllable, and in this way, as Thompson explains, time becomes money: "Those who are employed experience a distinction between their employer's time and their 'own' time. And the employer must use the time of his labour, and see it is not wasted: not the task but the value of time when reduced to money is dominant. Time is now currency: it is not passed but spent." ${ }^{\prime 59}$ When time-oriented labor becomes counted time, it makes work time accountable and evaluable, with the historical side-effect that it renders forms of labor that are not compensated monetarily, such as household and care work, traditionally (and even today) largely performed by women, invisible. ${ }^{60}$ This alliance between money and time, as Peters notes, rests on their being paradigm cases of "logistical media," or those media that "establish the zero points of orientation." ${ }^{n 1}$

In establishing a grid of temporal structure capable of containing and coordinating diverse practices in time, the calendar and clock have functioned to lift time out of the necessity of particular reference and produced what French historian Paul Ricoeur calls "anonymous time." ${ }^{62}$ Anonymous time, for Ricoeur, is a mediating temporality between phenomenological experience and worldly time; it functions to situate and compare subjective and objective temporalities, giving temporal place both to inner experience and external events. Reading Alfred Schütz's influential phenomenological account of intersubjectivity, Ricoeur argues that the division of social time into anonymous categories of "contemporaries, predecessors, and successors" initiates a temporal logic that forms a bridge between "lived time and universal time." ${ }^{\prime 3}$ The succession of generations, socially experienced as the "replacement of the dead by the living," ${ }^{64}$ constitutes a third time between 
inner subjective reality and the physical time of the world, out of which time becomes a matter of roles. People in their radical singularity can never replace one another in their phenomenological experience of time, a point that Martin Heidegger emphasizes in calling phenomenological time one's "ownmost possibility," 65 but they can step into vacated stations. This ability to assume specific temporal roles, first established in the psychological relationship of contemporaries, predecessors, and successors, is accelerated in the temporal coordination of industrial labor through the clock's division of work processes into replaceable tasks. Network temporalities today greatly expand these logistical functions and their anonymizing proclivities, as can be seen in ride-sharing services such as Lyft and Uber that, even as they track individual riders and drivers, treat them as anonymous data points to be algorithmically paired.

Indeed, anonymous time is crucial to timesharing companies, such as Lyft, Uber, and Airbnb, which automate both monetary transactions and clock and calendar time. Rides and rooms are not exchangeable with one another totally but are instead exchangeable by categories, anonymously. The driver is not treated as a singular, irreplaceable being but instead as a class of car, a set of reviews, an anonymous anchor for a constellation of ratings. Similarly, an Airbnb rental location is generalized, departicularized, made anonymous, and submitted instead to ratings, reviews, and other data points. Anonymous time is in this way constructed out of the unique possibility of precise addressability. Although such anonymity seems merely coincident with temporal organization, it proceeds from time management in a very radical way. Its freedom to accept multiple diverse phenomenological actors is founded on an exacting system of computed temporality.

\section{Technological Microtime}

Over the last two centuries, time media have increasingly come to operate on microtemporal levels. In this process, temporal infrastructures have come to more finely divide calendar and clock time, operationalize them, and establish the structuring grids necessary for a new density of action moments. For while calendrical moments, such as years, months, and days, are efficacious in calculating events such as the rise and fall of empires, the beginning and end of wars, or the course of a lifetime, they are inadequate for calculating the clock time of hours, minutes, and seconds. More minute and finely tuned temporalities require still more refined technologies of temporal measure, such as Jimena Canales has explored in the nineteenth century's invention of the "tenth of a second." ${ }^{66}$ Instruments such as 
chronoscopes, myographs, and photographic cameras, which operated beneath the temporal thresholds of human perception and reaction, carried remarkable epistemological significance. ${ }^{67}$

In their attempts to study the processes of sense perception, nineteenth-century experimental physiologists turned equipment such as telegraphs into instruments for measuring the microtemporal dynamics of muscle activity and the transmission of nerve impulses within living organisms. Hermann von Helmholtz' measurements of the velocity of nerve impulses and Matthäus Hipp's reaction time experiments, for example, gave rise to a new understanding of reality as being radically constituted by the conditions of temporal perception ${ }^{68}$ Such physiologists realized that the temporal experience of living things is determined by their respective temporal thresholds of perception and reaction, and thus the quality of temporal perception came to be understood as a function of quantitative values. Accordingly, the temporal category of the "present" was to be understood as determined by its appropriate sphere of action, the decisions that calculate its order of magnitude in the interplay between reaction times, transmission times, and processing times. Microtemporal actions and technological speed-up would thus be seen as yielding less quantitative than qualitative and thus aesthetic effects.

In 1860, the Baltic German entomologist Karl Ernst von Baer captured this relation eloquently in a series of thought experiments by demonstrating the relativity of temporal perception depending on the number of "moments" a perceiving subject is able to distinguish in a given interval. For instance, he stated that a human being taking in 1,00o instead of the usual $\sim 10$ moments per second would perceive a waterfall as a quasi-stable object, comparable to how we perceive the growth of plants, while events such as gunshots would appear as traceable movements (similar to the perception of cinematic slow-motion). On the other extreme, a subject with just one moment per day or even per month would perceive the sun not as a slowly moving object but, due to the extreme time-lapse, as a glowing ring, just like people would normally perceive a piece of coal fixed to a string swirling around in a circular fashion. Emerging from the same temporal regime of microtime, cinematography produced out of the temporal succession of photographs the illusion of visual movement. ${ }^{69}$ Notably, von Baer framed his ideas more than a decade before the famous chronophotographic experiments by Eadweard Muybridge and Étienne-Jules Marey, which precipitated motion pictures and demonstrated the synthesizing effects of microtemporal technologies. Since these experiments in the nineteenth century, the sciences of astronomy, psychology, and microphysics, as well as the various media industries of 
film, radio, and television, have constructed their own microtemporal infrastructures to enable and govern the actions within these domains beyond human sense.

The emergence of microtemporal technologies has correspondingly given rise to another form of temporal politics, to what we refer to as the politics of microtime. In order for technologies capable of recording, transmitting, and reproducing sounds and images to become media, they need to be articulated to one another and organized into larger social and economic systems..$^{70}$ Consequently, the political contestations of microtime are waged over temporal machine standards and media formats, such as motion picture frame rates, audio playback speeds, television line numbers, screen refresh rates, and compression standards for both audio and video, all of which pattern time on scales below the temporal resolutions of human perception and cognition and yet perform the necessary work of rendering human and nonhuman actions compatible..$^{71}$ Another example might be the 60 $\mathrm{Hz}$ standard of the North American power grid, which allows different technologies and devices to make use of the same resource and participate in the same industry. In this way, temporal standards serve as what Susan Leigh Star and James Griesemer call "boundary objects," or artifacts that enable and govern modes of cooperation between heterogeneous technologies and social worlds. ${ }^{72}$

The necessity of coordinating human and nonhuman time can be seen most fundamentally in the different ways traditional clock time and microtime are counted. Time systems that have a direct human interface, such as the calendar and clock, tend to use reference points that align with human experience and the necessities of human reckoning, while those time systems below the thresholds of human perception operate on the metric system. The Russian and French Revolutions attempted to institute nonreligious calendar systems that largely failed because of their lack of intuitive connection with the celestial rhythms of people's lives. The explanation is relatively simple. While the metric system is excellent for mathematical calculation, it is much less amenable to effortless counting and subdividing by human minds unequipped with paper and pencil. Sexagesimal (based on 60) and duodecimal (based on 12) systems on the other hand use superior composite numbers, with hours in a day being split into two duodecimal halves divisible by $2,3,4$, and 6 , and sexagesimal minutes and seconds adding divisors of 5 and 10, making them easily calculable at a glance. It is on the basis of time systems having no necessary human interface, however, that we owe much of contemporary technology. The industrialized acceleration of digital switching over the last five decades 
has not only transformed computers from machines of calculation and data processing into media, in the traditional sense of audiovisual technologies, but also rendered them increasingly as decision makers and thus autonomous nonhuman actors in the temporal flow of the real world. On the operational level, most general-purpose digital computers are based on technologies of short-term memory as opposed to long-term storage, a temporal logic Wendy Chun has termed the "enduring ephemeral," 73 which describes random-access operations of reading, writing, and deleting information that must be performed with considerable speed. The processual necessity to refresh, which forms the temporal basis of computing from computer memory and hard drives to bitmapped graphics and databases, makes possible an endless process of reading and rewriting. ${ }^{74}$

The time-criticality of computers, their ability to act in real-time or in the temporal flow of the world, is further predicated on technological reconciliations between external time windows and internal processing time. The principles and technologies of "timeliness," reliant as they are on the exponential growth of processing power captured in Moore's Law, are crucial to understanding digital temporalities, whether at play in the hardware of computers or in the interactions of networks. Indeed, technological speedup forms the very basis of "smart machines" and other forms of artificial intelligence applications. The range of qualitative tasks and actions that an algorithm can perform, such as listening, speaking, playing chess, detecting faces, or driving a car, largely depends on the number of calculations that can be made in a given critical time window. Time is still money, but in light of today's digital capitalism, characterized by big data analysis, algorithmic trading, and the mining of cryptocurrencies, it is especially the investments in microtime and the massive exploitation of data processing infrastructures that foster the contemporary imaginaries of value extraction. Given that digital devices increasingly engage as nonhuman actors and decision-makers in the real world, we need to consider the temporalities on which their "smartness" stands.

The focus of this volume is on this unique extension of technological microtime enabled by the universal medium of computation, which we refer to as digitally networked time. Digital time is marked by its universality and thus its ability to be extended into new domains of communication and action. It is the baseline possibility of temporal coordination between networked technologies that possess their own unique temporal orders. Like the internet itself, this temporal network is distributed and layered; it makes few restrictions on the types of time that can exist and enables programs and apps to institute independent interfaces of time. 


\section{Layout of the Volume}

The book is divided into four thematic sections, beginning with the holistic concerns of media philosophy and passing into topical considerations of temporal regimes on the micro, meso, and macro scale. The papers of the first section, "Media Philosophies of Time Patterning," investigate the specific ability of media to suspend the course of time and how they pattern time to make interventions, as nonhuman actors, in the present, past, and future, on the level of both technical phenomena and human decision-making. John Durham Peters takes up Kittler's view of technical media as means of "suspending irreversibility" and argues that this capacity for reversibility "is the necessary condition of repeatability, transmission, and data storage." In an essay that builds on arguments from his book The Marvelous Clouds, Peters ponders the phenomenological ironies of time's irreversibility, which exists, like music, in a constant dynamism of disappearing. Thus he reopens the question of media ontology as crucially a question of time. Gabriele Schabacher takes a media-theoretical approach to the question of care in analyzing the energies and labor practices necessary to maintain technological infrastructures, introducing a typology of four infrastructural care practices: repair, maintenance, abandonment, and repurposing. Yuk Hui, drawing on the phenomenology of Edmund Husserl and Martin Heidegger, as well as their more recent take-up by Bernard Stiegler, seeks to understand the unique new futurity coming into visibility with the rise of predictive technologies. He argues, provocatively, that a new category of temporal experience is currently taking hold, what he calls tertiary protention, or a future that issues not from one's own subjective projection but instead from a socially and technologically constructed projection imposed from without in the form of artificial intelligence. Wolfgang Ernst concludes the section by placing recent developments within a deeper set of time-critical operations involved in media infrastructures, focusing on "the basic layer of bit processing on the Internet." Delving into the operative dimensions of media infrastructures, Ernst directs attention to the microtemporal processes that are their sine qua non, using the example of the "Ping" signal as a time-critical signal of internet logistics.

Following from Kittler's determination of technical media as technologies that operate below thresholds of human sensory perception and cognition, the papers of the second section, on "Microtime," focus on media technologies that move beyond even the physiological and cognitive requirements for displaying textual and audiovisual information to alter and manipulate data in these inaccessible intervals. Isabell Otto shows in her analysis of current debates about abolishing the leap second that digitally networked 
media reveal "the fundamental relativity of each regularity of time." Taking as a starting point the video diary app Leap Second as a concrete example of the plurality of time experience, she argues for an understanding of the multiplicity of time measurements. The leap second makes a further appearance in Geoffrey Bowker's chapter "Life at the Femtosecond." Going back to Charles Babbage, Bowker roots the computer industry's drive for technological acceleration in the simple fact, as stated by Babbage, that although machinery cannot be built into unlimited space, it can run through unlimited time. Thus having not world enough, but time, computers traffic in speed. Addressing operations taking place at the femtosecond, or the very limits of technological microtime, Bowker asserts that, although they fall well below human perception, they are nevertheless "real in their consequences." Florian Sprenger focuses in on a particular area where the density of machinic action that can be performed in the blink of an eye has created extraordinary new levels of complexity, tracing the logic of microtemporal interventions in Tesla's advanced driver-assistance systems. These automated driving systems, by necessity, make decisions about future events that escape human sense. As Sprenger puts it, "the autonomous car brakes before the incident." It calculates time and speed to predict possible futures, such as a collision, opening up important questions about the politics of machine decision in these inaccessible intervals. Andrew R. Johnston, in his contribution on Google's DeepMind project, notes that these new levels of complexity come at a price. Researchers in machine learning are increasingly confronted with the problem of rendering the computational technologies they work with accessible. In particular, the efforts by Google's researchers toward accessibility have landed on the need for visualization, turning to video games from the Atari 2600 system, such as Qbert and Space Invaders, as ways of providing visual feedback for machine-learning test runs. Moreover, the focus on test runs in the development of machine learning systems reveals contemporary shifts in software engineering where programmers enter into new relations of care and coaching with increasingly autonomous algorithms.

Where the second section focuses on material changes and technical objects, the third, "Lifetimes," turns a lens toward the lived experiences of human beings as they interact with and work to maintain network infrastructures. Nicole Starosielski returns to the cable systems and network infrastructures that formed the basis of her book The Undersea Network to address the embodied experiences of the infrastructure operators who maintain these systems. Two crucial new concepts emerge out of this analysis: the idea that speed is always grounded speed in the sense that it 
relies on the temporal rhythms and safe passages of human bodies, and the problem of temporal irruptions that occur when the ground disrupts system speed. Through these, Starosielski directs our attention to the everyday experiences and politics that underlie digital networks, the often-omitted "soft temporalities" of hardwired infrastructures. In a related investigation, Marisa Leavitt Cohn argues that the overall emphasis in recent years on the materiality of software has neglected "the temporal dimension of this materiality—how software ages, decays, obsolesces." Drawing on her ethnographic work with engineers and software developers on a long-term space project, Cohn examines how aging software becomes unmistakable in its materiality and how it is feminized and pathologized for being material. Software shows up as an "unruly body of code" in its passage through different iterations, prompting a reckoning with its material history. In this sense, the felt materiality of code is a product of time, revealing the ideological forces that treated it as immaterial in the first place. In this way, Starosielski and Cohn also connect back to Schabacher's consideration of human laborers as caregivers for nonhuman actors. James Hodge considers how the temporal dynamics specific to network platforms open themselves up to entertainment, both anxiety inducing and fun. Three online artifacts come under Hodge's watchful eye and incisive analysis: Brian Eaton's artwork the Memento Mori Clock, the "This Is Fine" meme, and a YouTube video entitled I Put Wii Music over a Final Destination Death Scene. Through these artifacts, Hodge considers how media creators and viewers reclaim the demanding, machinic temporalities of contemporary infrastructures, which through digital preemption both short-circuit anticipation and proliferate experiences of anxiety, to make them humanly meaningful again. Concluding the section, Sumanth Gopinath traces the emergence of the digital wristwatch in the 1970s to show how designers used sound-in the form of "beeps" - to connect these devices to the human sense realm, an industry practice that has continued into the era of cell phones and smartphones.

The final section, "Futures," concerns the ways technologies reach into the future and order new regimes of time, commanding near-term actions and provoking dystopic and utopic visions of their power. Alexander Monea performs a media genealogy of the historical entanglement of vision and attention in the discourse on eye tracking. Connecting this to contemporary developments in eye-tracking technology, he argues that we may soon experience very undesirable new arrangements of the attention economy from digital platforms to smartphone apps where ads are able to stop playing when you look away from the screen. Eva-Maria Nyckel studies Amazon's anticipatory shipping model through an analysis of the company's patent 
filings. Nyckel argues that efforts to reduce shipping latencies and better forecast consumer demand have pushed logistical services to use predictive technologies that not only speculate about future events but also serve to build the infrastructure for their arrival. Amazon's anticipatory shipping model confirms that the industry's adherence to the dictum "time is money" is pushing it into developments where "time is media" and where effective media are the future of the medium of money. Andreas Sudmann examines artificial neural networks (ANNs) as predictive systems to illustrate the fundamental importance of analyzing this technology in terms of its temporal dimensions. One aim of his essay is to show how an investigation of the temporal infrastructures of modern ANNs also contributes to a more substantial discussion of their political challenges, such as can be seen in the labor of crowdworkers, hired via platforms like Amazon's Mechanical Turk, for labeling and producing the massive amounts of learning data for ANNs. Concluding the volume with a reading between Kittler's argument that technical media reorder time independently of human input and Stiegler's stance that technically mediated time is central to the experience of human time, Britt Paris looks at how the NSF-funded Named Data Networking (NDN) protocol—a possible successor to the current TCP/IP network protocol—reconciles "social concepts of time with computational and architectural constraints in network design." Paris draws on firsthand interviews with NDN researchers in her examination of how user-facing temporal experiences take second place to the imperative speed-up of information transmission.

\section{Notes}

1. Peter Conrad, Modern Times, Modern Places (London: Thames \& Hudson, 1998), 9 .

2. Hartmut Rosa and William E. Scheuerman, eds. High-Speed Society: Social Acceleration, Power, and Modernity (Philadelphia: Penn State University Press, 2009); and Hartmut Rosa, Social Acceleration: A New Theory of Modernity (New York: Columbia University Press, 2013). It is also worth acknowledging Paul Virilio's longstanding work on technological acceleration. See, e.g., Virilio, Speed and Politics: An Essay on Dromology, trans. Mark Polizzotti (New York: Semiotext(e), 1986). For works on accelerationism see Robin Mackay and Armen Avanessian, eds., \#Accelerate: The Accelerationist Reader (Falmouth, UK: Urbanomic Media, 2014); and Nick Srnicek and Alex Williams, Inventing the Future: Postcapitalism and a World Without Work (London: Verso, 2015). 
3. See, e.g., Jonathan Crary, 24/7: Late Capitalism and the Ends of Sleep (New York: Verso, 2013).

4. Emily Keightley, ed. Time, Media and Modernity (London: Palgrave Macmillan, 2012), 4, 201.

5. Sarah Sharma, In the Meantime: Temporality and Cultural Politics (Durham, NC: Duke University Press, 2014), 20.

6. Markus Krajewski, The Server: A Media History from the Present to the Baroque (New Haven, CT: Yale University Press, 2018), 339.

7. See Deborah Cowen, The Deadly Life of Logistics: Mapping Violence in Global Trade (Minneapolis: University of Minnesota Press, 2014). Also, for an excellent exposition of the scalar dimensions of media infrastructures, see the introduction to Lisa Parks and Nicole Starosielski, eds., Signal Traffic. Critical Studies of Media Infrastructures (Urbana: University of Illinois Press, 2015).

8. See, e.g., Jane Bennett, Vibrant Matter: A Political Ecology of Things (Durham, NC: Duke University Press, 2010); Ian Bogost, Alien Phenomenology, Or, What It's Like to Be a Thing (Minneapolis: University of Minnesota Press, 2012); Richard Grusin, ed. The Nonhuman Turn (Minneapolis: University of Minnesota Press, 2015); Bruno Latour, Pandora's Hope: Essays on the Reality of Science Studies (Cambridge, MA: Harvard University Press, 1999) and Reassembling the Social: An Introduction to Actor-Network-Theory (Oxford: Oxford University Press, 2005); and Jussi Parikka, A Geology of Media (Minneapolis: The University of Minnesota Press, 2015).

9. Bennett, "Systems and Things: On Vital Materialism and Object-Oriented Ontology," in The Nonhuman Turn, 223-239.

10. This is evidenced, for instance, by how contemporary online platforms produce distinct forms of device-specific "realtimeness." See Esther Weltevrede, Anne Helmond, and Carolin Gerlitz, "The Politics of Real-Time: A Device Perspective on Social Media Platforms and Search Engines," Theory, Culture \& Society 31, no. 6 (2014): 125-150.

11. Noble reveals how search engine results reproduce racist and sexist attitudes from the cultures in which they operate and reinforce those attitudes. See Safiya Umoja Noble, Algorithms of Oppression: How Search Engines Reinforce Racism (New York: NYU Press, 2018).

12. McIlwain breaks down his story into two parts, detailing the untold stories of Black entrepreneurs and innovators, who developed "black software" for the personal computing revolution and early years of the internet, and the adverse side of this history where new computing technologies were put to the task of fortifying racial hierarchies. See Charlton D. McIlwain, Black Software: The Internet and Racial Justice, from the AfroNet to Black Lives Matter (Oxford: Oxford University Press, 2019).

13. Benjamin covers a wide range of racial biases in technical systems, from campus architectures to online mapping tools, and outlines a set of coding practices to counteract the power of this "New Jim Code." Ruha Benjamin, 
Race After Technology: Abolitionist Tools for the New Jim Code (Cambridge, UK: Polity, 2019).

14. See, e.g., Friedrich Kittler, "Real Time Analysis, Time Axis Manipulation," trans. and with an introduction by Geoffrey Winthrop-Young, Cultural Politics 13, no. 1 (2017): 1-18.

15. See, e.g., Eva Horn, "Editor's Introduction: There Is No Media," Grey Room 29 (2008): 7-13; Erkki Huhtamo and Jussi Parikka, eds., Media Archaeology: Approaches, Applications, and Implications (Berkeley: University of California Press, 2011); Parikka, What Is Media Archaeology? (London: Polity, 2012).

16. Wendy Hui Kyong Chun, "The Enduring Ephemeral, or the Future Is a Memory," Critical Inquiry 35, no. 1 (2008): 148-171; Wolfgang Ernst, Digital Memory and the Archive, ed. Jussi Parikka (Minneapolis: University of Minnesota Press, 2013); Mark B. N. Hansen, Feed-Forward: On the Future of Twenty-First-Century Media (Chicago: University of Chicago Press, 2015); Adrian Mackenzie, "The Technicity of Time: From 1.oo oscillations/ sec to 9,192,631,770 Hz," Time \& Society 10, no. 2-3 (2001): 235-257; Florian Sprenger, The Politics of Micro-Decisions: Edward Snowden, Net Neutrality, and the Architectures of the Internet, trans. Valentine A. Pakis (Lüneburg: Meson Press, 2015); Axel Volmar, ed., Zeitkritische Medien (Berlin: Kadmos, 2009).

17. For literature, Paul Ricoeur, Time and Narrative, 3 vols., trans. Kathleen Blamey and David Pellauer (Chicago: University of Chicago Press, 1984, 1985, 1988). For film, Gilles Deleuze, Cinema 2, trans. Hugh Tomlinson and Robert Galeta (Minneapolis: University of Minnesota Press, 1994); and Mary Ann Doane, The Emergence of Cinematic Time: Modernity, Contingency, the Archive (Cambridge, MA: Harvard University Press, 2002).

18. Ernst, Digital Memory and the Archive.

19. See Volmar, Zeitkritische Medien, 25 .

20. See Erhard Schüttpelz, "Infrastructural Media and Public Media," Media in Action 1, no. 1 (2017): 13-61; Ulrike Bergermann, Monika Dommann, Erhard Schüttpelz, Jeremy Stolow, and Nadine Taha, eds., Connect and Divide: The Practice Turn in Media Studies (Berlin and Zürich: Diaphanes, 2020).

21. Media scholars have shown heightened interest in infrastructure studies in recent years in part because the growing ubiquity of digitally networked platforms, the global distribution of information, and the media ecologies of "smart cities" have shattered the idea of media being single devices or products that can be understood without recognizing the larger systems or environments that enable them. A survey of important texts includes Parks and Starosielski, Signal Traffic; John Durham Peters, The Marvelous Clouds. Toward a Philosophy of Elemental Media (Chicago: University of Chicago Press, 2015); and Nicole Starosielski, The Undersea Network (Durham, NC: Duke University Press, 2015).

22. Susan Leigh Star and Geoffrey C. Bowker, "How to Infrastructure," in Handbook of New Media: Social Shaping and Consequences of ICTs, ed. Leah A. Lievrouw and Sonia Livingstone (London: SAGE Publications, 
2002), 151-162; Lauren Berlant, "The Commons: Infrastructures for Troubling Times," Environment and Planning D: Society and Space 34, no. 3 (2016): 393 .

23. Keller Easterling, Extrastatecraft: The Power of Infrastructure Space (New York: Verso, 2014), 13.

24. Paul N. Edwards, "Infrastructure and Modernity: Force, Time, and Social Organization in the History of Sociotechnical Systems," Modernity and Technology, ed. Thomas J. Misa, Philip Brey, and Andrew Feenberg (Cambridge, MA: MIT Press, 2003), 186.

25. For the recent interest in questions of maintenance and repair, see Steven J. Jackson, "Speed, Time, Infrastructure. Temporalities of Breakdown, Maintenance, and Repair," in The Sociology of Speed, ed. Judy Wajcman and Nigel Dodd (Oxford: Oxford University Press, 2017), 169-205; and Andrew L. Russell/Lee Vinsel, "After Innovation, Turn to Maintenance," Technology and Culture 59, no. 1 (2018): 1-25.

26. Bruno Latour, "The Berlin Key or How to Do Words with Things," in Matter, Materiality and Modern Culture, ed. P. M. Graves-Brown (London: Routledge, 2000), 10.

27. Karl Marx, Capital, vol. 1, trans. Ben Fowkes (New York: Vintage, 1977 [1867]), 130. Marx writes: "As exchange-values, all commodities are only greater or smaller amounts of congealed labor-time."

28. Michel Serres with Bruno Latour, Conversations on Science, Culture, and Time, trans. Roxanne Lapidus (Ann Arbor: University of Michigan Press, 1995), 45, 60.

29. For more on cultural techniques of synchronization, see Christian Kassung and Thomas Macho, eds., Kulturtechniken der Synchronisation, Kulturtechnik (München: Wilhelm Fink, 2013).

30. Our understanding of technological temporalities as being segmented into micro, meso, and macro scales matches closely the tripartite division of time advanced by Fernand Braudel. However, the differences between our model and Braudel's should also be seen to signal important shifts in human and technological temporalities in a world dominated by networked digital media. See Fernand Braudel, "History and the Social Sciences: The Longue Durée," in On History, trans. Sarah Matthews (Chicago: University of Chicago Press, 1980 [1958]).

31. Serres, $5^{8}$.

32. Peters, Marvelous Clouds, 244.

33. S. Hultberg, "Some Observations on Systems for Automatic Acquisition and Reduction of Nuclear Data and a Preliminary Report on the Computer System Trask," Nuclear Instruments and Methods 34 (1965): 127.

34. Gordon E. Moore, "Cramming More Components onto Integrated Circuits," Electronics 19 (April 1965), 114-117.

35. James Carey, "Technology and Ideology: The Case of the Telegraph," in Communication as Culture: Essays on Media and Society, rev. ed. (New York: Routledge, 2009), 168. 
36. Judy Wajcman, Pressed for Time: The Acceleration of Life in Digital Capitalism (Chicago: University of Chicago Press, 2015), 3.

37. Obviously, integrated circuits use "wires" only in a metaphorical sense.

38. Other imaginative efforts include Daniel Hillis' Clock of the Long Now and the performance of John Cage's As Slow as Possible over the course of 639 years at a church in Halberstadt, Germany.

39. Peters, Marvelous Clouds. See also Thomas Macho, "Zeit und Zahl. Kalender und Zeitrechnung als Kulturtechniken," in Bild, Schrift, Zahl, ed. Sybille Krämer and Horst Bredekamp, Kulturtechnik (München: Fink, 2003), 179-92.

40. Harold Innis, Empire and Communications (Oxford: Oxford University Press, 1950). See also Innis, The Bias of Communication (Toronto: University of Toronto Press, 1951). As Innis showed, "time-binding," or durable, media have played a considerable role in the growth and maintenance of large political and economic bodies such as empires.

41. Émile Benveniste, "Language and Human Experience," Diogenes 13, no. 51 (1965): 1-12. Benveniste elaborates three conditions of calendars, namely initiating, directing, and measuring, the first of which involves the calculation of an axial moment: "All calendars share common characteristics which identify the basic conditions which they must fulfill. They take off from an axial moment which provides the zero point of the computation" (5).

42. Paul Ricoeur, Time and Narrative, vol. 3. (Chicago: University of Chicago Press, 1988), 107.

43. Ricoeur, 109.

44. Benveniste, 4. A common translation of Benveniste's temps chronique, as for instance in the translation of Ricoeur's Time and Narrative, Vol. 3, renders the term "chronicle time," and in many ways this translation better captures in English its sense of time as a succession of events. Benveniste himself, however, suggested its translation as "chronic time." Etymologically, the term "chronic" entered late Middle English as a cognate of the French chronique and meant simply "of time, concerning time," a meaning reaching back to the Greek khronikos, from kronos. Nevertheless, its later connotations of disease and addiction might helpfully express the adverse underside of the temporal structure of events.

45. Benveniste, 6 .

46. Lewis Mumford, Technics and Civilization (New York: Harcourt, Brace \& Company, 1934), 14.

47. Mumford, 15 .

48. James Henry Breasted, 1935. "The Beginnings of Time-Measurement and the Origins of Our Calendar." The Scientific Monthly 41 (1935): 289-304.

49. Peters, Marvelous Clouds, 1.

50. Eviatar Zerubavel, "The Standardization of Time: A Sociohistorical Perspective," American Journal of Sociology 88, no. 1 (1982): 11. See also his books Hidden Rhythms: Schedules and Calendars in Social Life (Berkeley: University of California Press, 1985), The Seven Day Circle: The History and Meaning 
of the Week (Chicago: University of Chicago Press, 1989), and Time Maps: Collective Memory and the Social Shape of the Past (Chicago: University of Chicago Press, 2012).

51. Zerubavel, "Standardization of Time," 3.

52. Mumford, $17-18$.

53. Mumford, 13 .

54. Mumford, 15 .

55. See Rosa, Social Acceleration.

56. E. P. Thompson, "Time, Work-Discipline, and Industrial Capitalism," Past and Present 38 (1967): 56-97.

57. Thompson, 73 .

58. Thompson, 73.

59. Thompson, 61 .

6o. See Susan Leigh Star and Anselm Strauss, "Layers of Silence, Arenas of Voice: The Ecology of Visible and Invisible Work," Computer Supported Cooperative Work (CSCW) 8, no. 1-2 (1999): 9-30.

61. John Durham Peters, "Calendar, Clock, Tower," in Deus in Machina: Religion and Technology in Historical Perspective, ed. Jeremy Stolow (New York: Fordham University Press, 2013), 42.

62. Ricoeur, 112.

63. Ricoeur, 105 .

64. Ricoeur, 109.

65. Martin Heidegger, Being and Time, rev. ed., trans. Joan Stambaugh (Albany: SUNY Press), 252.

66. Jimena Canales, $A$ Tenth of a Second: A History (Chicago: Chicago: University of Chicago Press, 2009), 3.

67. See also Soraya de Chadarevian, "Graphical Method and Discipline: Self-Recording Instruments in Nineteenth-Century Physiology," Studies in History and Philosophy of Science 24, no. 2 (1993): 267-291.

68. See Laura Otis, "The Metaphoric Circuit: Organic and Technological Communication in the Nineteenth Century," Journal of the History of Ideas 63 , no. 1 (2002): 105-128; Henning Schmidgen, "Of Frogs and Men: The Origins of Psychophysiological Time Experiments, 1850-1865," Endeavour 26, no. 4 (2002): 142-148.

69. Karl Ernst von Baer: Reden gehalten in wissenschaftlichen Versammlungen und kleinere Aufsätze vermischten Inhalts, Erster Theil. Reden (St. Petersburg: Schmitzdorff, 1864), 237-284. Reprint in Volmar, ed., Zeitkritische Medien, $45^{-59}$.

70. See Jonathan Sterne, The Audible Past: Cultural Origins of Sound Reproduction (Durham, NC: Duke University Press, 2003).

71. See Marek Jancovic, Axel Volmar, and Alexandra Schneider, eds., Format Matters. Standards, Practices, and Politics in Media Cultures (Lüneburg: meson press, 2020).

72. Susan Leigh Star and James R. Griesemer, "Institutional Ecology, Translations, and Boundary Objects: Amateurs and Professionals in Berkeley's 
Museum of Vertebrate Zoology, 1907-39," Social Studies of Science 19, no. 3 (1989): 387-420. See also Elaine K. Yakura, "Charting Time: Timelines as Temporal Boundary Objects," Academy of Management Journal 45, no. 5 (2002): 956-970.

73. Chun, "Enduring Ephemeral," 148.

74. See also Ernst, Digital Memory and the Archive.

\section{About the Authors}

Kyle Stine teaches Film and Media Studies at Johns Hopkins University. His writings on cinema and technology have appeared in Critical Inquiry, Discourse, Grey Room, and the Journal of Cinema and Media Studies.

Axel Volmar is a Postdoctoral Fellow at the Collaborative Research Center "Media of Cooperation" at the University of Siegen. He is author of Klang-Experimente: Die auditive Kultur der Naturwissenschaften 1761-1961 and co-editor of Format Matters: Standards, Practices, and Politics in Media Cultures and Interrogating Datafication: Towards a Praxeology of Data. 


\section{Part I}

Media Philosophies of Time Patterning 



\title{
1. The Suspension of Irreversibility: The Fundamental (and Futile) Task of Media
}

\author{
John Durham Peters
}

\begin{abstract}
This essay explores the media-theoretical and time-critical implications of the facts that we all will die and that we move irreversibly forward through time. It ranges broadly across several thinkers (such as von Baer, Eliot, Kittler, and Borges) and situations (thermodynamic decay, being stuck in traffic, and the rarity of randomness).
\end{abstract}

Keywords: media theory, time, thermodynamics, Kittler, von Baer, irreversibility

We all know what time is, said St. Augustine, until we start to ask what it is, in which case we really have no idea. Such a mixture of self-evidence in the long shot and bafflement in the close-up is not usual in philosophical inquiry. Most things that we know or think we know crumble under closer inspection. Socrates enjoyed terrorizing Athenian notables by demonstrating this fact. We all know that all people are mortal — that point is easy to get. But that I am going to die - that is much harder. Everyone must die; I am not everyone; therefore I am not going to die - this is the sort of warped syllogism that we all live by! The more basic the theme, the more the philosophical and existential stakes diverge. I want to explore the media-theoretical and time-critical implications of the facts that we all will die and that we move irreversibly forward through time.

Let's start with the hypothesis of Karl Ernst von Baer, the nineteenth-century Estonian nobleman and founder of the field of embryology, that there is a correlation between an organism's length of life and its sense of time's

Volmar, A. and K. Stine (eds.), Media Infrastructures and the Politics of Digital Time: Essays on Hardwired Temporalities. Amsterdam: Amsterdam University Press, 2021 DOI 10.5117/9789463727426_CHо1 
passage and duration. In a lecture on, among other things, entomology from 1860 - and thus too soon to take on board the Darwinian revolution - von Baer notes the ineradicable human habit of positing ourselves as the measure of time and space and of using our bodily proportions to serve as cosmic measurements, with the result that we can never get big enough to see everything. ${ }^{1}$ A second, he conjectures, was originally a heartbeat, and he thinks that our experience is potentially dividable into six to ten events per second, though he renounces the effort to specify the universal minimal interval for sensory experience. ${ }^{2}$ Here he picks up a theme beloved of phenomenologists and psychologists: how long is the now?

From this heuristic of sensory allotment-six to ten impressions per heartbeat - von Baer then goes on to consider rabbits and cows. Here the hypothesis gets a little goofy. Since cows have a pulse that is twice as slow, and rabbits twice as fast, their experience must run slower and faster, respectively, than ours. From this he weaves to the general point that any organism's experience of nature is a function of its physiology and lifespan. Thus creatures with different life spans would have a very different physics and astronomy than ours: a person with a thousand-fold acceleration of experience (and thus consequent shortening of life) would live through one phase of the moon; nights would stretch out into seasons enduring almost a year; trees (if in summer) would never lose their leaves and water never grow firm into an icy solid. A person with an even shorter lifespan of 42 minutes would never know that grass and flowers were not everlasting, and the "entire organic world would appear to be lifeless" (262). Von Baer is interested in varying the axis of temporal perception such that our eyes, and especially our ears, could stretch in their powers of discernment. (His time-lapse and slow-motion fantasy is obviously resonant for the pre-history of cinema.) If our life were short enough - that is, if our perception of fine slices of time were acute enough - we might even be able to hear light, though of course we would no longer be able to hear what we now hear. (He seems to assume that sensory acuity covaries with temporal perception; he doesn't imagine our music software that allows pitch-shifting while holding the time axis invariant.) A universe of vibrations currently inaccessible to us would appear if the temporal structure of our organisms changed: we might see sound, hear heat, or even listen to the music of the spheres as the planets oscillate (263).

Von Baer not only speeds up the elementary unit of perception but also slows it down. What sort of universe would a person live in who had a pulse a thousand times slower than ours-i.e. someone destined to live not 80 but 80,000 years? Von Baer notes that an 80,00o thousand-year person 
would barely perceive the shuttling between day and night and might never recognize that it gets completely dark, since an entire year would take place within " $311 / 2$ " heart beats (you have to love the precision). The rumbling bass tones that move the universe along would finally become audible. In his Principles of Psychology (1890) William James riffs on the time-lapse effects of von Baer's speculations about the slow-pulse person: "mushrooms and the swifter-growing plants will shoot into creation so rapidly as to appear instantaneous creations; annual shrubs will rise and fall from the earth like restlessly boiling water springs; the motions of animals will be as invisible as are to us the movements of bullets and cannon-balls; the sun will scour through the sky like a meteor ..."3

The punch-line of von Baer's speculations is that such varieties of temporal experience are not merely hypothetical but probably already exist in the animal kingdom of protozoa and insects, pigeons and bees. Just as there are huge ranges in spatial perception-a microscope can transform a puddle into a universal ocean - so there are radically varying scales of temporal experience. The best standard, says von Baer, is the biggest-everything is always too small. In some ways our knowledge is bound by our temporal mode.

George Eliot (Mary Anne Evans) clearly knew of von Baer as both an acquaintance of one of his most dogged disciples (Herbert Spencer) and as one of the best informed people about German thought in nineteenth-century England. In her masterwork Middlemarch (1871-1872) she describes the insulation of our self-knowledge as a kind of existential comfort. "If we had a keen vision and feeling of all ordinary human life, it would be like hearing the grass grow and the squirrel's heartbeat, and we should die of that roar which lies on the other side of silence. As it is, the quickest of us walk about well-wadded with stupidity." In contrast to von Baer's discontent with our inevitably puny point of view, she found something to commend: sensory dullness saves us from a fatal cosmic roar. Charles Sanders Peirce took the same lesson, that a relatively short lifespan provides us with narrative materials for meaning-making, such as beginnings and endings, and prevents us from the wreckage that infinity would inevitably bring. "If man were immortal he could be perfectly sure of seeing the day when everything in which he trusted should betray his trust, and, in short, of coming eventually to hopeless misery. He would break down, at last, as every good fortune, as every dynasty, as every civilization does. In place of this we have death." ${ }^{5}$ If we weren't mortal, the universe might not make sense.

Such embedment in the finite definitely makes it hard to get a fix on the historical strangeness of the world that we live in today in comparison 
with the past. We have a necessarily myopic historical sense. As James said of a human life accelerated into a duration lasting less than an hour, "if born in winter, we should believe in the summer as we now believe in the heats of the Carboniferous era." A day blurs into a season blurs into an epoch depending on your quotient of mortality. This scale-dependent perception describes our historical near-sightedness quite well. We live within a rare geological moment within a rare geological moment, the upper holocene boundary. A pleasant spell of nice weather, known as the holocene, has enabled civilization as we know it, by providing cooperative natural conditions for settlement, agriculture, and large populations over the past ten thousand or so years. Since around 1800 , human population, capacity, wealth and carbon have exploded. From 1900 to 2000, cropland doubled, the human population quadrupled, the pig population went up ninefold, energy use went up sixteen times, and industrial output went up forty times. ${ }^{6}$ To von Baer's 80,000-year person, the industrial era would spring into being almost like a spontaneous creation, and to people born in the middle of it like us, it might seem second nature.

We live in THE moment, the human apex of planetary domination. The fact that we are alive is a tribute to the favorable natural and anthropogenic conditions for the flourishing of large human populations. If we were to roll the dice, chances are we would be born when we were. Somewhere between 5 and $10 \%$ of all human beings who have ever lived are alive right now, depending on your estimate of the total number of humans who've lived on earth (taking 107 billion as a standard estimate). In his novel 2001, which parallels the film, Arthur C. Clarke suggested that there were thirty dead people who had once lived for every person alive: "Behind every man now alive stand thirty ghosts." He was writing in 1968, when the human population was under 4 billion. If he were writing today when it is pushing 8 billion, he'd have to change it to fifteen ghosts. In five decades, the odds of being alive in this moment have doubled.

This fact has subtle warping effects on our perceptions. Our lives, our bodies, our minds, our visions of the world have all been shaped by time on a planet when there is a vast herd of domesticated human beings - who weigh about one third of the total vertebrate matter on earth, the other two thirds being accounted for almost entirely by animals that humans keep in order to eat. ${ }^{7}$ Those birds chirping outside, the whales in the sea, the feral dogs of Athens are all only a tiny fraction of vertebrate biomass compared with the anthropocentric part. Never has the planet been arranged like this. To us it seems as natural and eternal as daylight or dandelions would be to a 42-minute human. 
Consider a puzzler. Why is it that when you are sitting in traffic, you feel like you are always in the slowest moving of all the lanes? You feel that way in part because you probably are in the slowest moving lane. This perception is not a result of egocentric woe-is-me bias; the odds are that it is highly accurate. The slowest lane is the lane with the most cars in it. The odds are that you will be in the lane with the most cars. What you think of as your private perception or a spell of bad luck is in fact the infrastructural fact of your connection to larger populations and conditions than you can perceive. (In the famous words, being precedes consciousness.) Extraordinary geological conditions profoundly shape our everyday perceptions but our lives are too short to take those conditions as anything but normal. Our moment is both unique in human history, since for only a couple centuries has anything like this been possible, but also widely shared by billions. We have lots of synchronic, but little diachronic solidarity.

Media theory has a mixed mission. Partly, it aims to make the unconscious conscious, to bring out the thisness of a that and the thatness of a this (as Kenneth Burke said). But media theory also aims to defy any strong split of subject and object. Our consciousness converges with our conditions. The frustrated driver in the slow lane's view of the traffic is a function of where they are in it in the same way that our view of history and of the universe is a product of the history of the universe. The anthropic principle, to simplify, suggests that the conditions necessary to produce an observer predestine the kind of observable universe there could be. ${ }^{8}$ What we can know is deeply tied up with the processes that have produced us as observers. (This argument updates the German idealist claim found especially in Hegel and Schelling that history enables the collusion of subject and object since they are its common progeny.) The only universe in which we could exist, and period of the universe in which we could exist, is one that is biased — to invoke the ever-useful term from Harold Innis-in systematic ways. It would have to be old, spread out, and cold, but not too much. It would have to have a certain kind of chemistry, in our case, organic, which presupposes supernovas, the cosmic furnaces that forged by nucleosynthesis every element more complex than hydrogen and helium. The size and scale of the cosmos are in some way complicit or at least correlated with our ability to know them. Or, maybe better put, our minds are shaped by the same conditions that we are trying to observe.

On a cosmic scale, we live in a relatively brief moment in the history of the universe, and a brief corner of its diaspora, in which things stick together, in which houses cohere and trees thrive, on an earth in which matter is $10^{30}$ times more concentrated than the cosmic average. In the 
eons, the cosmos will degrade into diffuse boring nothingness, unable to stick together in any way. When the universe is $10^{18}$ seconds old, as it is now, a certain kind of knowledge is possible. The entropic cost of observation is not prohibitive. It is possible to discern causal chains. By the time the universe is $10^{107}$ seconds old, it will be out of thermodynamic and cognitive gas: assuming continued expansion (against a possible Big Crunch) each particle will be as far from every other as the current universe is wide. 9 Maybe von Baer should have applied his sliding time scale not to organisms but to the cosmos. If you imagine yourself with a millennial, million- or billion-year heartbeat, you can feel the future pulling the universe apart, and even your body; some of us, indeed, can already feel that. If we lived in a much later era of the universe, the nature of things would have changed enough to require a different kind of knowledge, mode of observation, and observer. The physical limits of the knowers are shared in some ways by the physical limits of the things to be known.

These reflections grow out of thermodynamic debates about order since the later nineteenth century. Does order belong to things or to mind? The point of Maxwell's demon, the famous thought experiment, was to turn entropy uphill by sorting out gas molecules into fast and slow. Maxwell's demon somehow knew where all the molecules were without exerting any effect on their behavior. Mind was invisible, aloof and yet somehow everywhere without interfering - rather like a parody of the god of mathematicians or of Descartes' thinking substance without extension, a point without materiality. ${ }^{10}$ The stakes were that this smart but physically non-intervening being could push entropy uphill and thus save the universe from the inevitable "heat death" of universal degradation. Clearly there was a cultural element here: the morality of husbanding energy well, masculinity under threat (as it chronically is), the waning coal supply, etc. for worried Victorians. ${ }^{11}$

Quantum physics put an end to the dream of a noninterventional omniscience. Knowledge and the behavior of subatomic particles turned out to be woven from the same fabric. How an electron behaves depends on how we look at it. As John von Neumann said, "An observation is an irreversible process. ${ }^{12}$ The more a system is monitored, the more it is tampered with. You can distort the behavior of a system by watching it - a fact that is not only true in culture, as Stanley Milgram, Michel Foucault and many others have shown, but in nature as well. The particles in the quantum panopticon monitor their monitors. There is no representing without intervening, to invoke Ian Hacking. ${ }^{13}$ Knowledge is physical. Norbert Wiener, who must count as one of the great thinkers of hardwired temporality, noted: "In nineteenth century physics, it seemed to cost nothing to get information." ${ }^{\text {"14 }}$ 
We know now just how expensive information is, both thermodynamically and in a more ordinary sense in our digitally-scraped lives.

Time, of course, is just as mysterious an entity as information. Is it a feature of the subject - time flies while you're having fun, or while your heart beat is faster, as von Baer thought_-or of the object-relentless clock time? Is time's arrow, its irreversible flow, just a function of entropy, of the fact that everything in the universe tends to move from a more ordered to a less ordered state? Is time the medium of entropy or its expression? Is time's irreversibility necessary or just highly probable? There is no physical law, in fact, saying that you cannot scoop up the precise same moles of water molecules that your cup just poured into the sea, but the odds are crushingly minute that you will. You could reconstruct the house that burnt down in the fire from its ashes: there is no dictatorial law saying it is impossible, only absurdly slight in probability. Maybe tables really do occasionally rise in the air due to fortuitous conjunctions of Brownian motion and maybe time occasionally, in coincidental convergences, lurches backwards or hovers briefly in slight hiccups.

I once wrote: "Ironically enough, just as physics was discovering irreversibility media engineered reversibility" but I no longer think it ironic. ${ }^{15}$ Time-reversibility in media shows the time-irreversibility of life. The great analog media of time-axis manipulation, phonography and film, showed that optical and acoustic data, once arranged into series, could be sped up, slowed down, or reversed in playback, but they also showed with fresh clarity that some natural processes cannot be reversed. It is remarkable how often cinema and sound-recording pop up in discussions of irreversibility. Wiener, for instance, in the famous opening chapter to Cybernetics on Newtonian and Bergsonian time, uses a nice film metaphor. He argues that astronomy is reversible: "The music of the spheres is a palindrome, and the book of astronomy reads the same forward as backward" (31). That is, a film of the planets, played backwards, would be possible according to Newtonian mechanics. "On the other hand, if we were to take a motion-picture photograph of the turbulence of the clouds in a thunderhead and reverse it, it would look altogether wrong. We should see downdrafts where we should expect updrafts, turbulence growing coarser in texture, lightning preceding instead of following the changes of cloud which usually precede it ..." (32).

A few years later, Karl Popper objected, saying that Newtonian physics also allows irreversible processes, using the example of "a film taken of a large surface of water initially at rest into which a stone is dropped." The reversed film will show the waves increasing instead of dissipating, flowing 
in rather than out, and culminating in the sudden appearance of a placid surface. As he drily notes, "This cannot be regarded as a possible classical process. ${ }^{16}$ Popper's point seems to be that entropy is not necessary, only highly probable, in keeping with his view of an indeterminate universe. Under some highly special or bizarre conditions, a stone might be able to spring out of a turbulent body of water, leaving it completely smooth: it is possible, only extremely improbable. Film is the sine qua non of both Wiener's and Popper's staging of reversibility. (Perhaps Newton's use of calculus to slice motion into asymptotically small intervals is already cinema avant la lettre, or its mathematical antecedent at least.)

Fortunately for media scholars, we have a robust tradition of theorizing the relationship between entropy and media, time and mortality that goes back to Friedrich Kittler's incandescent texts on time-axis manipulation and lightning and thunder. The first, "Real Time Analysis, Time Axis Manipulation" (1990), starts with the observation that the alphabet was the first technology of time-axis manipulation. Kittler notes that nature, unlike writing, does not recognize the copresence of full and empty slots. There are, that is, no yes-no, i.e., discrete, machines in nature-no placeholders, no decimal points or zeroes, no spaces between words. You can rearrange letters along their syntagmatic axis but you can't do that to natural phenomena. "You can certainly reverse the word LEBEN [life] and logically get the word NEBEL [fog], but not life itself, to say nothing of fog itself." (In English we can think of golf and flog or live and evil.) His examples are not by chance the core objects of the entropic imagination: life and weather. Life doesn't regenerate itself once it is dead; thunder does not go before lightning. These are one-way events. In discussing Georges Méliès's pioneering 1895 film on the mechanical butcher that shows a sausage turning into a pig, Kittler heralds: "Und die Auferstehung des Fleisches ward Anschauung." Geoffrey Winthrop-Young translates: "Behold the resurrection of the flesh!" This is an admirable solution to translating a phrase that echoes both the Gospel of John and the chorus mysticus from Goethe's Faust: "And the resurrection of the flesh became perception.” Unfortunately for Kittler's argument, no such thing occurs in the film, but as usual in Kittler, we shouldn't let the facts get in the way of an interesting idea: where only theology heretofore has dared contemplate such reversibility, now media theory steps forward. In a historically unprecedented way, analog media in the late nineteenth century "made for the first time contingent time-series events recordable." As with writing and the alphabet before, recording media opened up a realm of reversibility and play that is briefly exempt from the relentless grinding of real time. 
In a lecture on "Lightning and Series - Event and Thunder" first published in 2003 Kittler continues in his transcendental way. This is one of the most sublime and baffling texts I know on any subject. In it he tells a tale of three moments in the history of acoustic media:Zeus's thunder in ancient Greece, Father Mersenne's experimental cannonball blasts in the seventeenth century, and modern computers directly playing with the universe of frequencies thanks to Fourier analysis. (Sometimes it sounds as if Kittler has been reading von Baer.) The single stroke of the event/lightning cannot be perceived by humans due to the slowness of nervous propagation in our bodies; we have access only to the rumble of the vibrations, the series/ thunder that follows. The gods had lightning, but humans had thunder. But modern computation and mathematics bring together extremes that were separated in antiquity. We can't fly like Icarus into the Olympian range where gods read frequencies, but our computers can. Below the sensitivity of our ears lies thunder's frequencies; above the sensitivity of our eyes lies lightning. If what Kittler calls our "filters (eyes and ears, etc.)" could ascend or descend the ladder, they could play with light as we do with sound, with lightning as we do with thunder. (He gushes about the recent sonification of an earthquake in Kobe, Japan.) Any still image of sound such as a spectrogram necessarily implies a temporal dimension, typically along the $\mathrm{x}$-axis. In contrast, many images appear frozen in time. An image does not necessarily require a time-axis (though images can imply one, such as a blurred photograph). To the gods, however, every image would require a time-axis. If our eyes operated at god-like-computer-like-speeds, we'd know that every still visual image is also a slice of time. Pictures only pretend to freeze time for beings with slow sense organs. The old division of spatial image and temporal sound is just a species-specific result of the speed of our sense organs. Other species would see the time in every image or freeze sound into shape. Such a species, for Kittler, is the computer.

Kittler goes theological, or at least proposes what Foucault once called "an analytic of finitude": "as nature has consigned us to a finitely broad spectrum in her immeasurable range of frequencies so the old gods consigned us to finitely long lives in the time domain." Here human sensory limitation and mortality again become the marks of our condition, defined both thermodynamically and media-theoretically. Note the two sentences that begin the lecture: "Nothing is denied more to us temporal beings than to know time." The second one - the lecture was given to an audience of graduate students-is practical: "When have you read your sources enough to be confident of them?" Starting from a primal ban on human knowledge-his set-up reflects the Edenic scenario of knowledge the transgression of whose limits would make 
humans dangerously or excitingly like God-Kittler shifts to wondering when we will ever have the confidence to call our studies done. For him, our finitude in perception and knowledge go together with the irreversibility of nature and reversibility of media. As he concludes, with relevance to both any research project as well as to Heisenberg's uncertainty principle, "Every possible choice between being and time comes at the cost of knowledge."

Sybille Krämer astutely sees in time-axis manipulation the very heart of Kittler's thinking. She offers my thesis in a nutshell: "the most basic experience of human existence-and this is relevant because the human is, after all, a physical being - is the irreversibility of the flow of time. Technology is precisely the attempt to ward off (or charm) this irreversibility. In media technology, time itself becomes one of several variables that can be manipulated." ${ }^{\prime 7}$ In life, time goes in one direction only; in media, time can be stretched, compressed, reversed, yo-yoed, in short, edited. With media we can hopscotch through time, but in our bodies, we get one second older every second. Our techniques allow parallel processing, but we live according to the strict law of serial processing.

Hartmut Winkler brilliantly builds on Kittler and Krämer as well as Bernard Vief to make the point that temporal reversibility in media owes to spatialization. As he puts it in his book on processing, "media in general are machines that transcode space into time and time into space. ${ }^{18}$ Time axis manipulation operates by the transposition of sequentially occurring events into spatially ordered symbols, which then can be rearranged without cost. LEBEN becomes NEBEL without danger or expense. What Winkler calls "the geometry of time" occurs this way in alphabetic writing, in magnetic tape, in a vinyl LP, and internet streaming - in short, in any kind of data storage that enables a temporal playback of serially accessible data. Space here serves time. In the first of these, speech, which is a linear stream in time, is projected onto the space of the writing surface with the divisible corpuscles of letters. This is the fundamentally unnatural act of putting placeholders into a linear system. (Nothing is discrete in nature.) This argument is implicit in Kittler, specifically in his praise - which goes further in his grandiose final books on music and mathematics-of the ancient Greeks for having "placed the time of tones into space."19 He was thinking of how on a monochord, an ancient instrument, or Odysseus's bow, you could point to places—like frets on a guitar — that sounded the octave, fifth, and fourth. Here space marks sound. The only way to jump out of real time is to take advantage of space's ability to transcend time.

Winkler builds on the Brothers Grimm tale about the hedgehog and the hare who challenge each other to a race. The hare, though faster, always 
loses. The hedgehog has placed a second hedgehog, his wife who looks just like him, at the end of the furrow in which the race is to occur. It takes no time for the doubled hedgehog to get from one end to the other. The hedgehogs taunt the hare as the latter runs like lightning toward the finish line, "I am already here."20 The hedgehog is the principle of simultaneity, or of multiple copies pre-distributed in space, of parallel processing which costs no time, while the hare is the principle of transmission, or of serial processing, which always costs time. (Marisa Leavitt Cohn shows this acutely well in chapter 10.) The work of past time is recorded and available for instant access without the requirement of scrolling in real time. A nice point clinching Winkler's argument is buried in a footnote. A crowd is gathered. Two medics and a doctor are working on a man lying on the ground. Someone, when asked what happened, reports: he was dead. ${ }^{21}$ Making death past tense is the ultimate in time axis manipulation, and Kittler was right to think about resurrection here. To say that someone was dead is not just a grammatical puzzle, but the essence of TAM. No question, save perhaps the incarnation, is more important to Christian theology_or to Kittler's media theory.

It's important to note that we can't actually manipulate real time. Temporal and spatial fixity are not symmetrical. Playback is still new every time. Time is always mercilessly and blessedly disappearing. This is the point about time-axis manipulation being ultimately futile. You can't record sound; you can record instructions that allow you generate it anew every time in a form that is functionally but never completely identical. Sound as pressure or vibration will always be slightly different in each new instantiation. Kittler quotes Hegel's "merciless sentence" that sound exists by disappearing or disappears by existing. Recording media don't hold sound-they hold scripts that inscribe patterns for sounding-devices to perform, but once called forth, sound will dissipate once more. Sound is dissipation-no way around it. Light is too, but we are too dull to know better.

I recently proposed that cetaceans-dolphins, porpoises, and whales-model intelligence consigned to nonmaterial expression by their aqueous environment and lack of manipulative limbs. They live in matter, but cannot mold it. What matter is to dolphins, time is to us. Dolphins lack three-dimensional modeling; we lack four-dimensional modeling. Unlike such smart marine mammals, whose durable engineering is limited to moving mud around on the ocean floor or pulling things around, humans have developed a vast array of durable media, material moldables into which we can imprint our schemes. Among the greatest of all human technical achievements is the ability to record the data of happenings in spatial form 
and then spin them back later into real-time. The playback of course takes time and occurs in time but we cannot, in the end, capture time. No material ultimately catches and molds real time. We can only impose symbols serially onto spatially manipulable media. Any time control erodes just as quickly as the dams the dolphins build. We cannot get anything to stick for good in real time; at best we use symbolic machines to enable reversibility under special conditions. We have to convert time into space to manage it. We can only "write" events on some lasting spatial substrate that play them back with some kind of fidelity. The best we can do is substitute space for time, and firm for fluid matter. We lack any medium of four-dimensional plasticity.

What if we could mold real time? It might sound like bliss to be able to recall our sweetest moments in all their fullness, and have memories as strong as the experience. It also might be a complete hell, if pain would recur again and again in memory as intensely as it took place in experience. (The name for such repetition is trauma, a psychological fact intimately connected with playback technology. ${ }^{22}$ ) The lack of four-dimensional manipulability is at once a cursed and handsome condition. That we hurtle forth serially in time, irreversibly, means that we are compelled to choose. Irreversibility is actually tied up with conditions of meaningfulness. (This echoes Peirce's point that death is our defense against complete annihilation.)

Everything we do is completely improbable. It is not that our deeds lack meaning but that it is impossible for us to act in anything but a meaningful way. Every act is a choice against infinitesimal odds. Take the act of writing. At the most minimal, take 26 letters plus a space: 27 options are found for every parking spot on the line of writing (a typical keyboard allows more like 90 characters, if you include the shift key, and then there are all the special characters.) Type 56 characters $\left(=27\right.$ to the $56^{\text {th }}$ power or about 10 to the 8oth) and you have already met the number of protons in the universe; type 70 characters (about 10 to the 10oth, that is, a googol) and you are well beyond the highest estimate of how many elementary particles there are in the universe (10 to the $97^{\text {th }}$ ). Type 2000 characters, around a double-spaced page, and you have "unimaginably" succeeded the size of the universe. This of course is Borges's Library of Babel..$^{23}$ The possibilities multiply no less staggeringly with speech. With 44 phonemes in English, for instance, as soon as you articulate 61 of them you surpass a googol of possibilities. (There is no grammar for action, but the same vastness prevails.) There are so many options, but each choice narrows the next. If you write the letter $Q$, odds are that the next letter will be a U. We can write Iraqi or QWERTY and still make sense, but the possibilities for a meaningful choice shrink to a needle's eye the further you go. Thermodynamics says that there are many more ways 
that things can be chaotic than organized. Muddle has near infinite versions, tidiness only a few. We can't help but be meaningful. The burden we bear is not, as the pop existentialists thought, the universe's lack of meaning, but the inability to stop making sense. Our symbolic machines—spoken, written, optical and acoustic, digital — lift us out of time's flow at the price of being obedient to their rules. ${ }^{24}$ We walk through time unconsciously crushed by the barometric pressure of uncountable possibilities. And we can only say, do, see, or hear anything in the moment between past and future. $\mathrm{O}$ tempora, $\mathrm{O}$ media!

\section{Notes}

1. "Welche Auffassung der lebenden Natur ist die richtige? und wie ist diese Auffassung auf die Entomologie anzuwenden?" (1860), Reden gehalten in wissenschaftlichen Versammlungen und kleinere Aufsätze vermischten_Inhalts (St. Petersburg: Verlag der Kaiserlichen Hofbuchhandlung H. Schmitzdorff, 1864), 3 vols. Facsimile reprint 2 vols. (New York: Arno, 1978), 1:239-284. The media-theoretical implications of this lecture are nicely treated by Stefan Rieger, "Der dritte Ort des Wissens: Das Gedankenexperiment und die kybernetische Grundlagen des Erhabenen," Zeitkritische Medien, ed. Axel Volmar (Berlin: Kadmos, 2009), 61-80, esp. 66-72.

2. He was in tune with the later nineteenth-century interest in the tenth of a second as an interval of perception: see Jimena Canales, $A$ Tenth of a Second (Chicago: University of Chicago Press, 2009).

3. The Principles of Psychology (1890; New York: Dover, 1950), 1:639.

4. Middlemarch (1871-2; New York: Penguin, 1994), 194.

5. Charles Sanders Peirce, "The Doctrine of Chances," in Chance, Love, and Logic, ed. Morris R. Cohen (Lincoln: University of Nebraska Press, 1998), 72.

6. John R. McNeill, Something New Under the Sun: An Environmental History of the Twentieth-Century World (New York: Norton, 2000), 360-361 and passim.

7. Vaclav Smil, Harvesting the Biosphere (Cambridge, MA: MIT Press, 2013).

8. John D. Barrow, The Constants of Nature (New York: Vintage, 2002), chapter 8 .

9. William Poundstone, The Recursive Universe: Cosmic Complexity and the Limits of Scientific Knowledge (Mineola, NY, 2013 [1985]), 163.

10. See James Gleick, The Information: A History, A Theory, A Flood (New York: Pantheon, 2011), chap. 9 .

11. Crosbie Smith and M. Norton Wise, Energy and Empire: A Biographical Study of Lord Kelvin (Cambridge: Cambridge University Press, 1989).

12. In Poundstone, Recursive Universe, 67 .

13. Ian Hacking, Representing and Intervening (Cambridge: Cambridge University Press, 1983). 
14. Norbert Wiener, The Human Use of Human Beings: Cybernetics and Society, 2nd ed. (Boston: Houghton Mifflin, 1954), 29.

15. John Durham Peters: The Marvelous Clouds (Chicago: University of Chicago Press, 2015), 310.

16. K. R. Popper, "The Arrow of Time" (Letter), Nature 177 (17 March 1956): 538.

17. Sybille Krämer, "The Cultural Techniques of Time Axis Manipulation: On Friedrich Kittler's Conception of Media," trans. Geoffrey Winthrop-Young, Theory, Culture, and Society 23 (2006): 93-109, 96, trans. slightly modified.

18. Hartmut Winkler, Prozessieren: Die dritte, vernachlässigte Medienfunktion (Munich: Fink, 2015), 12.

19. Friedrich A. Kittler: "Blitz und Serie - Ereignis und Donner," in Zeitkritische Medien, ed. Axel Volmar (Berlin: Kadmos, 2009), 157; see also Musik und Mathematik. Vol. 1: Hellas, Part 1: Aphrodite (München: Fink, 2006).

20. "Der Hase und der Igel," Die Märchen der Brüder Grimm: Vollständige Ausgabe (Augsburg: Goldmann, 1981), 559-61.

21. Winkler, Prozessieren, $237 n 356$. This brilliant chapter is available in English translation at homepages.uni-paderborn.de/winkler/hase-e.pdf.

22. Amit Pinchevski, Transferred Wounds: Media and the Mediation of Trauma (New York: Oxford University Press, 2018).

23. William Goldbloom Bloch, The Unimaginable Mathematics of Borges' Library of Babel (New York: Oxford University Press, 2008). See also my "Resemblance Made Absolutely Exact: Borges and Royce on Maps and Media,” Variaciones Borges 25 (2008):1-23, https://www.borges.pitt.edu/bsol/documents/Peters.pdf.

24. The guarantee of encryption in non-quantum computing-i.e. the inability to step sideways out of time for hedgehog like parallel calculations-is the same as the proof of insincerity in interpersonal ties: finitude, that is, the scarcity of time.

\section{About the Author}

John Durham Peters, Maria Rosa Menocal Professor, Department of English, Yale University, New Haven CT, author most recently, with the late Kenneth Cmiel, of Promiscuous Knowledge: Information, Image, and Other Truth Games in History (Chicago, 2020). 


\title{
2. Time and Technology: The Temporalities of Care
}

\author{
Gabriele Schabacher
}

\begin{abstract}
By focusing the temporalities of care, the chapter analyzes a special relation between time and technology that underlies the making and persisting of media and infrastructures. I propose to differentiate between four types of care practices with corresponding different temporal patterns that are highly relevant for the functioning of technological systems in the past and present. First, the retrospective response to unforeseen interruptions (repair); second, the prospective routine procedure to prevent all forms of disorder (maintenance); third, a neglect of care that leads to devaluating infrastructure (abandonment) as well as - fourth-forms of revaluation in changing contexts (repurposing). Taking the new Berlin airport BER as an example, it will be shown that infrastructures exhibit different layers of temporality formed by these cyclic and repetitive processes of care and their transforming effects. Thus, even the performance of the most "hardwired," late modern technology systems is crisscrossed by temporal regimes that stem from older, non-modern temporalities of care.
\end{abstract}

Keywords: repair, maintenance, care, infrastructure, cultural techniques, abandonment

In this article, I analyze the relations between time and technology that underlie the making and persisting of media and infrastructures by focusing on the temporalities of care. I take up the notion of the hardwiredness of infrastructures in the sense of their consolidation and material embeddedness, raising the question of the practices responsible for achieving and maintaining technical structures. This implies understanding hardwiredness not as a fixed state of being of materially "wired" artifacts or digital infrastructures,

Volmar, A. and K. Stine (eds.), Media Infrastructures and the Politics of Digital Time: Essays on Hardwired Temporalities. Amsterdam: Amsterdam University Press, 2021 DOI 10.5117/9789463727426_CHO2 
but rather as a network effect with relative duration brought about by specific types of labor. Seen in this light, the effect of being "hardwired" appears not only in processes of industrialization and standardization since the nineteenth century but also in older, premodern cultural techniques responsible for the conservation of things. I understand caring activities in the sense of a certain type of constant, repetitive, and comparably slow work that tends to be invisible and naturalized in the mundane routines of everyday life. The advantage of such a perspective for media studies is that it enables insight into the various temporalities of infrastructures, which, as I argue, form a vertical layering of different ages within one and the same infrastructure, leaving several strata to be uncovered.

Recent research in science and technology studies, urban studies, architectural studies, and the history of technology has shifted attention to processes of upkeep and repair as highly relevant to the functioning and stabilization of organizations and infrastructures. ${ }^{1}$ However, questions of repair and upkeep are less addressed with respect to the functioning of digital infrastructures. ${ }^{2}$ For this reason, I take up the insights of repair studies, especially as developed in the fields of architecture and urban studies, in order to show their relevance for a discussion of hardwired infrastructures under digital conditions. Repair studies analyzes the care of physical infrastructures, such as buildings and transport systems, and acknowledges the materiality of those things, people, and codes out of which also "the digital" is made, whether cables, workflows, or programs. Such a perspective has already been productive in studying the work places and labor practices of early computing, for example in analyzing the so-called software crisis of the 1960 s as "essentially a maintenance problem."

The concept of care has recently been addressed with regard to ethical implications and the type of work it generates, together with the question of who cares for whom and the affective relationships that go along with this practice. According to Maria Puig de la Bellacasa, the three dimensions of care- "labor/work, affect/affections, ethics/politics"—are distributed in all relational situations. ${ }^{4}$ Care work can refer to people (including the care for oneself), 5 things and artifacts, but also to machines and infrastructures. Thus, the gender bias ${ }^{6}$ care work implies, the "invisible work" 7 it goes along with and the "tacit" ${ }^{8}$ knowledge of carers are relevant aspects for an analysis of care work, whether this relates to humans or the realm of material objects. ${ }^{9}$ Care is in general closely linked to concern ${ }^{10}$ and draws on affective relations between human and nonhuman actors. Latour's study of the transport system Aramis discloses the affective relationship with technology (the love of technology) as the most important aspect for accomplishing or giving up 
on a project. ${ }^{11}$ Only on the basis of an attachment to a certain thing can an affective relation of care be built. ${ }^{12}$

Studies of care stress two kinds of temporalities involved in caring practices. On the one hand, they refer to the fragility of the material world and processes of decay; on the other hand, they point to the work side of the problem, that is, the array of continual "reordering micro-processes."13 Steven Jackson claims that the temporalities of maintenance and repair blend "the unruly timelines of things." Drawing an analogy between the care of the human body and the repair and maintenance of objects, he describes a temporal relation in which the care of things involves "a staying with in time and place," that means, an adjusting of one's own time to "other temporal flows and processes, including the temporalities of breakdown and decay." Repair-as-care, according to Jackson, implies opening and tying oneself to the "rhythms, flows, and timeliness of another." ${ }^{14}$

Considering these ideas, I propose to differentiate between four types of practices that concern the care for technology and, correspondingly, four different types of temporal patterns. First, the activities of restoring-especially practices of repair - that respond to unforeseen events, ranging from catastrophic situations to ordinary breakdowns and malfunctioning, and rebuild a previous state of affairs. With respect to their temporal logic, these activities can be understood as a retrospective form of care. Second, the practices of maintenance, upkeep, and regular service of technical systems that are intended to prevent all forms of disorder by routine and planned procedures of control. Insofar as these practices are directed toward the future of technology, I will understand them as a prospective form of care. Third, the devaluation of infrastructures through decay and deterioration. These processes exhibit an inversion or negative form, a lack of care characterized by neglect and indifference. Fourth, and sometimes as a result of decay, processes of revaluation and repurposing that I understand as a redirection of care by reusing an existing infrastructural setting.

Although the distinctions between the four different types of caring activities are not clear-cut, I take them to be useful for analyzing temporal regimes of hardwiredness. This offers a perspective on care practices that concentrates on their status as cultural techniques and demonstrates that maintenance carries forward a non-modern notion of care stemming from the cultural techniques in the sense of cultura. In doing so, the article wants to accentuate two general aspects. First, in light of these various temporal patterns of care, hardwired infrastructures appear less as linear, enduring, stable objects and systems than as cyclical and repetitive processes of formation and transformation. Even the most hardwired, late-modern, 
high-technology systems are crisscrossed by different temporal regimes, especially on the level of micro practices that stem from older, non-modern, and, to some degree, more organically and culturally embedded temporalities of care. This becomes obvious in particular with regard to what I will call "maintaining-as-waiting" (warten). Second, different layers of temporality and care practices exist within any single infrastructural setting. These various temporalities and "ages" of infrastructures produce effects of interference, accumulation, and repetition because regimes of work, affective relationships, juridical decisions, lifetimes of components, to name only a few, are not distributed in a coordinated way with regard to actors and goals.

\section{Layered Temporalities: The BER project}

The different layers of temporality inherent in a given material infrastructure can be illustrated by the well-known example of the Berlin Brandenburg Airport. The BER had been under construction since 2006, after its initial planning in 1995. The opening had been postponed several times since 2011 (see Table 2.1). The last target for the official opening date was October 2020, but this again had been called into question because of an internal (leaked) TÜV Rheinland report of 2019 revealing serious safety deficiencies in important technical installations and suggesting an opening not before 2021. ${ }^{15}$ In the public, the BER is known as a case of severe mismanagement, corruption, and poor construction planning and execution. The already long list of construction flaws became longer every day (the TÜV report lists 11,519 technical flaws altogether), and the costs for not bringing the airport into operation amounted to about 36 million euro per month. The Flughafen Berlin Kosten website displayed the rising costs per second in real time. ${ }^{16}$ By 2020 the whole enterprise had cost the taxpayer up to 5.3 billion euro already and would have increased to an estimated 7 billion euro by 2025 .

Yet from an infrastructural perspective, the BER project demonstrates not only mismanagement but also the heterogeneity of the socio-technical "actor-world" in question in a paradigmatic way, ${ }^{17}$ bringing together materials, political parties, contracting companies, reports, and investigations, ${ }^{18}$ as well as considerations of environmental impacts, increases in passenger traffic, design flaws, and costs. The BER project shows the failure to come to mutual and simplifying translations to align all these heterogeneous elements in one direction, namely the completion of the construction project. Instead, each entity operates as a full mediator of the whole process, following its own directions. ${ }^{19}$ 
Table 2.1. Postponement of BER opening dates

\begin{tabular}{ll}
\hline Announcement & Opening \\
\hline September 5, 2006 (original) & October 30, 2011 \\
June 25, 2010 & June 3, 2012 \\
May 7, 2012 & March 17, 2013 \\
October 27, 2012 & October 27, 2013 \\
January 1, 2013 & on/after 2014 \\
January 8, 2014 & on/after 2015 \\
February 24, 2014 & on/after 2016 \\
May 14, 2014 & on/after 2017 \\
December 1, 2014 & 2nd half of 2017 \\
January 21, 2017 & July 10, 1905 \\
December 15, 2017 & October 31, 2020 \\
\hline
\end{tabular}

From Wikipedia, Berlin Brandenburg Airport, https://en.wikipedia.org/wiki/Berlin_Brandenburg_Airport, accessed November 11, 2020.

With regard to the temporalities of infrastructures, the BER is interesting in at least three respects. First, it demonstrates the difficulty of consolidating and stabilizing a project at all, that is, coming to a (temporary) state of "hardwiring." The BER project failed even to arrive at this point of upkeeping an achieved stability, as the heterogeneity of citizens, organizational actors, laws and prescriptions, environmental organizations, and material and technical components could not be aligned long enough to complete the project in the first place. Second, the time it took to build the infrastructure itself encompassed processes of decay. The passenger information monitors already had to be replaced because they had reached the end of their lifetime.$^{20}$ In the airport's underground train station, several "ghost trains" had to be run each day to provide fresh air and prevent mold from accumulating. ${ }^{21}$ Regimes of planned obsolescence, as seen in the case of the monitors, interfered with the longevity of the construction process; cycles of maintenance had to be executed to simulate usage, although the structures were not officially open to the public. Third, the time that passed itself altered perspectives on the future of the project. New calculations of projected passenger traffic, for instance, now demand a restructuring of the whole airport area. The so-called "master plan 2040"22 calls for a rewiring, so to speak, of the whole project before it was even completed, merging the current areas of the Berlin Schönefeld Airport with the unfinished BER and adding a "midfield" and other buildings to create an even bigger airport. The map combines existing buildings, buildings planned until 2030, and desired future buildings, thereby showing the site as a "contested gathering of many 
conflicting demands" where processes of design constantly interfere with what already has been built. ${ }^{23}$

On a more general level, the example shows different layers of temporality within a single infrastructure. According to Steward Brand, in How Buildings Learn, the very idea of architectural permanence is misleading. Instead, the adaptability of any architecture has to be seen as a continual flow of transformations. "All buildings grow" and age, says Brand, but "different parts of buildings change at different rates." ${ }^{24}$ Brand differentiates between the layers of "site," "structure," "skin," "services," "space plan," and "stuff."25 Whereas the geographical setting, or site, of a building is long-lasting, the layer of services, such as electrical wiring, plumbing, sprinkler system, and ventilation, must be renewed every seven to fifteen years. This level of services caused severe problems in completing the BER, as monitors, cables, and even safety helmets became obsolete during the process. The "shearing layers of change" have the effect of the building "tearing itself apart." ${ }^{26}$ With regard to the BER, we can see shearing layers of different time scales not only with respect to the physical dimensions of the infrastructure but also their socio-technical aspects, such as bureaucratic procedures, prescriptions, investigations, and planning.

The temporal regimes involved in the BER example concern, on the one hand, infrastructures of temporality, that is, the governing of time through plans, management routines, and maintenance cycles. On the other hand, the example illustrates the temporalities of infrastructure, that is, the different lifespans of the involved entities, the transforming effects of flaws, mistakes, and corruption on the project as a whole and the (organic) processes of decay and repurposing. A notable example of repurposing is that the uncompleted airport became a tourist attraction. One could book tours via bus or bike and, as of March 2017, 1.55 million visitors had been shown around the airport. ${ }^{27}$ A perspective on the relations of technology and time can therefore provide for a fruitful perspective on infrastructural networks, adding to spatial, topological, and environmental approaches insights on the ephemerality of their existence.

\section{Repair}

As we have seen with respect to the BER project, different layers of temporality exist within the same infrastructural setting. In the following, I describe in more depth the four practices of care outlined above, starting with the practice of repair. 
How one assesses the importance of repair in the present it is first of all a question of perspective. Under conditions of scarcity, repair has been ubiquitous for a long time in history, ${ }^{28}$ and it still is in countries of the Global South, whereas in Western industrialized countries "the continuities between the hidden and ongoing cultures of repair that characterize urban life outside of catastrophic states ... tend to be dramatically underplayed." ${ }^{29}$ As a consequence, infrastructures tend to be regarded as "hardwired," that is orderly, fixed, and stable. However, repair activity is not less relevant in the present, but only less visible. The international repair movement (repair cafés, the website iFixit, for example), ${ }^{30}$ while claiming a fundamental "right to repair" and taking up ideas from discourses on sustainability, commonism, and the do-it-yourself-scene, ${ }^{31}$ focuses on household items (toasters, printers) or consumer products (iphone, ipad) and their planned obsolescence rather than on machines and infrastructures. More fundamentally, Steven Jackson calls for a "broken world thinking," a perspective on the principal fragility and vulnerability of the material world that takes its "always-almost-falling-apart" as the starting point for a rethinking of repair activities. ${ }^{32}$

With respect to the temporalities of infrastructure, two aspects have to be emphasized. First, the constitutive ex-post-character (Nachträglichkeit) of repair (re-parare, in the sense of a reconstruction ${ }^{33}$ ), which is responsible for the epistemic quality of repair, as knowledge of breakdowns or accidents only becomes evident in the aftermath of an irritation. ${ }^{34}$ For this reason, I call this practice retrospective care. Second, and nevertheless, the grade of belatedness and visibility differs. Roughly speaking, Western societies believe and imagine themselves to be living in more stable environments (despite all the makeshift solutions in daily life), whereas people in the Global South engage openly in repair as a ubiquitous, mundane activity.

Regarding the BER example, the obsolescence of products forced repair work. Besides the 750 monitors mentioned, 16,849 fire detectors had to be exchanged at a cost of 1.6 million euro because go percent of them had reached the end of their permitted service life. This turned out to be quite lucrative for the respective firms, as they could build the airport several times, so to speak. 35

\section{Abandonment}

Abandonment refers to the processes of devaluation that technologies and infrastructures undergo as a result of a lack of care. ${ }^{6}$ When infrastructures 
are not cared for, they decay. Or more precisely, other processes take over. While the BER was not used as an airport, grass could grow in places where there was otherwise intense traffic. The heterogeneous actors were not aligned in one direction, but follow their own paths.

The concept of decay raises questions concerning the status of materiality and its "hardwiredness." Regarding the material ecology of subway signs, for example, the workers did not experience the signs' "materiality" but were immersed in a "malleable material flux" of different material properties, including the walls, metal brackets, and signboards, as well as screws, plugs, and glue cement. ${ }^{37}$ From an architectural and urbanist perspective, such a flux can be understood as "architecture's 'life' and 'death." Instead of relying on architecture's attested "material durability," this means to concentrate on the relation of architecture to "decay, deterioration, and destruction." 38 In reference to Michael Thompson's analysis of the complex processes of valuation and devaluation of things, ${ }^{39}$ Stephen Cairns and Jane M. Jacobs highlight "matter" and "mattering," that is, the dimension of materiality and the processes of valuation, as two concepts to explain the "relative durability" of built structures, which in their opinion is linked to a specific temporality:

Architecture's relative durability does not exempt it from the principle of mutable value, but it does ensure that architecture generally "circulates" via processes of reinvestment, restoration, and revaluation - more slowly through its ebb and flow. As a consequence, buildings are regularly out of time — unused, unloved, unappreciated, devalued—but still very much in place. ${ }^{40}$

Infrastructural decay should not be seen simply as a natural by-product of the time passing. In her study on the aging infrastructure of a NASA space project, Marisa Cohn shows that it requires active work to bring about the end-of-life of such a huge system. Infrastructural decay, here, "is composed of multiple lifetimes of different parts of the system — hardware, software, code, organizational processes, programming languages, institutions, careers-all of which are entangled and are aging or obsolescing at different rates. ${ }^{141}$

With respect to the logic of abandonment, the specific obduracy of built structures seems interesting, as it serves to make visible the lack of care. An obsolete building can be understood as an "obduracy-in-obsolescence," being "in place but out of time."42 It cannot be made to disappear from sight in simple ways: "Unlike other waste objects, which can be managed or rendered invisible by being pushed into a garbage bin, stored in the attic, compacted in a landfill, or biodegraded, buildings often, resolutely and 
publicly, stay in view and in place regardless of their economic and public evaluations. ${ }^{13}$ This can also be seen at the BER. The buildings are physically there, they cannot be easily removed, although the expected increase in the number of passengers would demand another, bigger airport. So we only see the demolition (Rückbau) of selected parts. ${ }^{44}$

The distinction between different time scales of infrastructure provides a further perspective on the obduracy and stability of infrastructures. In his "multiscalar approach," Paul N. Edwards refers to the different temporalities of human life, history, and geology. ${ }^{45}$ Infrastructures exhibit stability and durability only on the level of human and historical times, whereas on geological or "long-term historical" scales infrastructures and nature present themselves as less discernible from each other, up to the point of their identification where "[N]ature is ... in some sense the ultimate infrastructure." ${ }^{m 6}$ Nevertheless, Edwards' view on infrastructure's fragile, ephemeral qualities is informed by an understanding of technical malfunctioning (he uses the concepts of "irregularity," "breakdown," etc.) rather than an interest in processes of decay and deterioration or material relics and ruins, which are already beyond a logic of functioning and purpose. However, certain organic processes represent symptoms of abandonment, such as the grass growing between the concrete joints at the BER, and can be seen as the interference of other time regimes within the logic of infrastructural becoming.

Processes of decay shift our attention from disturbance and disaster, as more or less discontinuous and abrupt events, to slower and often unnoticed temporal processes of change. Nevertheless, as any amateur gardener knows, nature reconquers man-made structures steadily. Processes of abandonment can therefore be seen as an inverted or negative form of care, characterized by indifference and a lack of concern.

\section{Repurposing}

In his autobiography, Roland Barthes recalls the allegory of the Argo as an object of continual, transformative processes of care:

A frequent image: that of the ship Argo (luminous and white), each piece of which the Argonauts gradually replaced, so that they ended with an entirely new ship, without having to alter either its name or its form. The ship Argo is highly useful: it affords the allegory of an eminently structural object, created not by genius, inspiration, determination, evolution, but by two modest actions (which cannot be caught up in any mystique of 
creation): substitution (one part replaces another, as in a paradigm) and nomination (the name is in no way linked to the stability of the parts). ${ }^{47}$

What Barthes invokes in the ship that the Argonauts permanently rework is the perspective on a processuality of technology and architecture that is barely noticeable, in that it draws on not radical upheaval or individual genius, but steady transformation. Although Barthes argues from a structuralist perspective that underlines the analogous and language-based quality of substitution within the same paradigm, it is nevertheless possible to think of a ship's material quality and the necessity to improvise with respect to repair and maintenance work due to scarce resources on the high seas.

This transformative aspect has also been addressed as technology's "fluidity" and "adaptability."${ }^{\text {} 8} 8$ With regard to mobile phone repair in Dhaka and Kampala, Steven Jackson argues, "the phone that emerges at the end is demonstrably not the same device." The work done on the mobile phone changes it: "The phone has become in effect a different object: new but not radically new, separated from and connected to its past by the forms of breakdowns, maintenance, and repair through which it has passed." ${ }^{m 9}$ The same is true for all the processes connected to the reworking of software and digital infrastructures, such as updating, and jumpering..$^{50}$ This work of transformation generally implies the need to improvise, that is, workarounds and makeshift solutions that include moments of bricolage, artisan tinkering, and creativity. ${ }^{1}$

Regarding the BER, one could say that the project did not stay the "same" over the time of its construction. Even more, it had not been "unique" from the beginning. For Latour, "to design is always to redesign," there is "something medial in design" so that it never creates ex nihilo..$^{2}$ Rather, artifacts are conceived as "complex assemblies of contradictory issues," disputed matters of concern, that we are still unable to design in all their complexity. ${ }^{53}$ Against this background, one could even say that the BER from the beginning is a project of re-designing, as it starts from "something that exists first as a given, as an issue, as a problem." ${ }^{44}$ And this "given" is Berlin's "airport situation," which the new BER is supposed to change for the better.

A further aspect regarding the transformative nature of care concerns the re-direction of its goals, the "creative" processes of adaptation and repurposing of artifacts and technologies toward other contexts. ${ }^{55}$ As the emphasis on first design neglects "the extraordinary life stories" of objects and technologies in other cultures, there is the need to account for 'creole' technologies" as fundamental parts of change: "[M]ost change is taking place by the transfer of techniques from place to place. ${ }^{m 6}$ 
Interestingly enough, such transformations not only solve problems but also generate new ones, which can be illustrated by a historical example. With reference to the difficulties of the shipment of plants at the end of the eighteenth century, Maren Mayer-Schwieger has shown that the legendary mutiny on the HMS Bounty was more or less a direct result of transformations on the ship. In an effort to prevent the breadfruit plants being shipped from dying, as had been the case in previous attempts at transporting live plants, radical measures were taken. Primarily, the captain's cabin became the storage space for hundreds of flowerpots. This arrangement severely disturbed order aboard the Bounty, in particular, the hierarchical priority of the captain, and the resulting mutiny again led to a failure of plant transportation. ${ }^{57}$

Concerning the specific type of care in question, three aspects can be emphasized. First, processes of re-structuring necessarily belong to the usage of infrastructures and artifacts. Second, they can generate radical effects and conflicts because of the involved processes of de- and revaluation of certain elements of the network, such as the captain's cabin. And third, to focus on processes of re-structuring and re-purposing calls into question the assumption that there can be something like a "new" artifact. Something is new only in relation to what is already there. As for the temporal dimensions of care for technology, re-structuring processes can therefore be understood in terms of the redirection of care to new goals by re-using an existing and potentially devaluated infrastructural setting.

\section{Maintenance}

If we now take a closer look at the set of caring activities involved in maintenance, ${ }^{5}$ upkeep, and regular service of technical systems, we can see that caring for technology takes a prospective form, here, as it is directed to stabilizing the uncertain future of an artifact, technology, or system. Caring in this respect demands a focus on not only the functional relations to technology but also the "moral relations." 59 It seems as if the question of care can only be linked to technology if the latter is situated in an area "beyond" the realm of mere technical functioning.

Forms of maintenance often go along with an affective relationship to the maintained object, in particular on the level of micro practices. The subjects involved regularly develop a certain "sense" for the thing cared for, which provides a sort of diagnosing tool. The service operator responsible for Paris' water management system (SAGEP), for example, claims that the sluices 
of Paris' water supply had "talked" to him via the old servo-controls. ${ }^{60}$ In a similar way, some of the engineers who worked for decades within the aging Saturn mission spacecraft infrastructure were "accustomed to computing work that would be difficult to find still practiced elsewhere. ${ }^{\text {"1 }}$ What these examples illustrate is the fact that although control may be digitalized, the stabilization of the systems as a whole is dependent on procedures that refer to its material substrate as well as to the acquired skills of the caretakers (which again implies an essentially temporal dimension).

To elaborate on the specific temporality of maintenance and upkeep, the etymology of the word waiting (German warten) is of special interest. The verb warten in German has two meanings: first, to maintain, to upkeep; and second, to wait, to hold out. As I argue, it is this dimension of waiting that underlies and determines the temporal logic of maintenance processes ${ }^{62}$ In the Deutsches Wörterbuch, the most comprehensive dictionary of the German language, begun by the Grimm Brothers in 1854, we find an entry on warten of no less than 42 columns. The article describes the usage in the sense of waiting ("to await what is coming") only in the final part, while the preceding pages are centered on the relation between directed attention and the practices of guarding, watching, and caring. Interestingly, people are also said to be maintained ("Personen warten") in the sense of being cared for or administered. ${ }^{63}$ This includes caring for the sick, children, animals, gardens, and - more common to today's usage - objects. To speak of care with respect to things, the Grimm Brothers note, means to care for or just to deal with them constantly. ${ }^{64}$ This last mentioned aspect establishes a link between temporal and affective dimensions of maintenance, as this type of practice creates and is, therefore, embedded in a sort of habit.

The process of maintaining-as-waiting in the sense of the German warten denotes a specific form of concern characterized by attentiveness toward the object of care, be it a thing, a person, an animal, or, we can add, a machine. ${ }^{65}$ This attention takes the form of a close and constant contact with objects cared for. We thus deal with an activity that derives its temporality from the objects it follows in an effort at preservation. Referring to Heidegger's notion of dwelling as "sparing and preserving," 66 one could say that Beingin-the-World in the sense of dwelling can be understood as form of care for the environment as such.

In general, maintaining-as-waiting (warten) always proceeds in the present, but it is, at the same time, directed prospectively toward the future of the object cared for. This can be seen as a fundamental difference to the practice of repair that responds to a disturbance or, at least, irritation, and to forms of neglect of care that leave the former object of care to itself, 
whereas forms of repurposing may again involve a future for the object cared for. The practices of maintaining-as-waiting (warten) cultivate a type of concern tuned to preventing harm by regularly caring for objects and people, such as through the oiling and cleaning of machines and the proper nutrition and hygienic measures of people. The lifetimes of artifacts and people, in effect, coincide with the processes of their maintenance. Cost-intensive infrastructures, such as weapon systems, aircrafts, and busses, thus tend to live "eternally," if they are cared for in a proper way. This can be seen by the continued usage of taxis, busses, and even bicycles sorted out in Western societies in the Global South, ${ }^{67}$ or in the necessity to actively end their lifetimes. ${ }^{68}$ However, this longevity as an effect of maintenance practices reminds us of the basic fragility of things. Things do not "exist" in an uncomplicated way but only because of the work and care invested in them.

Although maintenance work can be described with respect to temporalities of different infrastructural entities, it is of course also relevant to point to the infrastructures of temporality that are in turn involved in processes of maintenance. Two aspects can be distinguished.

First, and in contrast to the possible longevity of artifacts and machines, consumer products have implanted limits that are known as planned obsolescence, and are conditioned to become cyclically out fashioned in increasingly shorter intervals. This phenomenon belongs to the logic of capitalist value creation, for which it would be fatal if objects were cared for in a relevant sense. This is one reason why today's call for "repairability"69 and "maintainability,"70 that is, the production of maintainable products (e.g., the Fairphone with interchangeable parts), ${ }^{71}$ did not manage to gain a deeper impact until now. Accordingly, the so-called life cycle management might be taken to amount to nothing more than the attempt to exploit each phase of life of the object (planned obsolescence inclusively). Although it may seem at first glance as the revival of older practices of re-using, known from an economy of scarcity, the idea of life cycle management is informed by cost-effectiveness and not by a logic to spare things. ${ }^{2}$ Nevertheless, also in the economic field, there are tendencies to see maintenance not as a "necessary evil," but rather as a potential to minimize material and energy consumption, ${ }^{73}$ controlled by "life cycle 'big data' analytics." ${ }^{74}$

Second, processes of technical maintenance are often informed by infrastructures of temporality. As we saw with the Berlin Airport example, these procedures follow certain cycles (for example, the necessary ventilation runs of the airport train). They are structured according to the logic of intervals of time and therefore dependent on a certain type 
of clocking that organizes their execution, repetition, and control. Seen in this light, maintenance procedures might also belong to the realm of planning, logistics, and management, and they might thus be adapted to a cybernetic logic of control.

\section{Conclusion}

In analyzing the hardwiredness of infrastructures, I have distinguished several layers of temporalities that go along with four different practices of care. I have discussed repair as an activity that aims to restore order retrospectively. While this represents the normal state of things in the Global South or under preindustrial conditions, Western industrialized societies often exhibit a belief in the stability of infrastructures. The phenomenon of decay, in turn, has been addressed as a form of abandonment that results from devaluation and lack of care, whereas processes of transformation and repurposing have been analyzed as complementary forms of revaluation and therefore redirections of care. For the activity of maintenance, I have assumed a dialectics of non-modern concepts (the logic of concern as a type of attentiveness to things in the form of waiting) and modern concepts (that integrate maintenance into logistical cycles of management).

Considering practices of maintaining-as-waiting (warten) of devices, machines, and large technical systems in particular, we get closer to basic processes within the realm of the organic, where cultura in the sense of care and concern for the soil in agriculture is important for the flourishing of plants, animals, and humans. Such a perspective highlights the logic and importance of cultural techniques to our modern understanding of culture, society, and technology. ${ }^{75}$ Maintaining-as-waiting (warten) would then refer to a specific "Eigenzeitlichkeit," or temporality, of things, demanding that one holds out and allows things to take their time. ${ }^{7}$

This also implies a non-modern understanding of maintainability, for which the general adaptivity of things is central: "Age plus adaptivity is what makes a building come to be loved."77 The possibility of modification is responsible for the adjustment of buildings and infrastructures to the changing conditions of time. ${ }^{7}$ Consequently, in terms of care, "a concern for maintainability translates material permanency into sociotechnical sustainability, and recognizes that stability, like reality, is 'an active verb."'79 We have to develop an understanding of "appropriate technology," where "fluid object[s]" remain open to change and dismantling during their development. ${ }^{80}$ Taken as matters of concern, things are on the move 
in the very basic sense of "moving project[s]." ${ }^{81}$ As I have argued, maintenance in the sense of waiting could then be seen as a set of extremely undervalued practices of care and concern that are important for the continued existence of culture, society, and technology, including the continued existence of digital cultures. From this perspective, hardwired infrastructures appear less as stable and durable systems than as ongoing processes of transformation. Even high-technology infrastructures, thus, are consisting of and crisscrossed by different temporal regimes that include non-modern, more organically and culturally embedded temporalities of care.

\section{Notes}

1. See for example Christopher R. Henke, "The Mechanics of Workplace Order: Toward a Sociology of Repair," Berkeley Journal of Sociology 44 (2000): 55-81; Stephen Graham and Nigel Thrift, "Out of Order: Understanding Repair and Maintenance," Theory, Culture \& Society 24, no. 3 (2007): 1-25; Steven J. Jackson, "Rethinking Repair," in Media Technologies. Essays on Communication, Materiality, and Society, ed. Tarleton Gillespie, Pablo J. Boczkowski, and Kirsten A. Foot (Cambridge, MA/London: MIT Press, 2014), 221-239; Stefan Krebs, Gabriele Schabacher, and Heike Weber, eds. Kulturen des Reparierens. Dinge - Wissen - Praktiken (Bielefeld: transcript, 2018); Andrew L. Russell/ Lee Vinsel, "After Innovation, Turn to Maintenance," Technology and Culture 59, no. 1 (2018): 1-25.

2. For notable exceptions see Neil Pollock et al., "Post Local Forms of Repair. The (Extended) Situation of Virtualised Technical Support," Information and Organization 19, no. 4 (2009): 253-276; Jason Farman, "Repair and Software: Updates, Obsolescence, and Mobile Culture's," continent. 6, no. 1 (2017): 20-24. With respect to new media's logic of constant actualization, see Wendy Chun, Updating to Remain the Same. Habitual New Media (Cambridge/London: MIT Press, 2016).

3. Nathan Ensmenger, The Computer Boys Take Over. Computers, Programmers, and the Politics of Technical Expertise (Cambridge, MA/London: MIT Press, 2010), 225. See also Thomas Haigh, "The Chromium-Plated Tabulator. Institutionalizing an Electronic Revolution, 1954-1958," IEEE Annals of the History of Computing 23, no. 4 (2001): 75-104.

4. María Puig de la Bellacasa, Matters of Care: Speculative Ethics in More Than Human Worlds (Minneapolis/London: University of Minnesota Press, 2017), 5.

5. Foucault, Michel, The Care of the Self. The History of Sexuality 3 (Harmondsworth and others: Penguin Books, 1990); see also Annemarie Mol, Ingunn Moser, and Jeannette Pols, eds. Care in Practice. On Tinkering in Clinics, Homes and Farms (Bielefeld: transcript, 2010). 
6. Emily K. Abel and Margret K. Nelson, eds. Circles of Care: Work and Identity in Women's Lives (New York: State University of New York Press, 1990).

7. Susan Leigh Star and Anselm Strauss, "Layers of Silence, Arenas of Voice: The Ecology of Visible and Invisible Work," Computer Supported Cooperative Work (CSCW) 8, no. 1-2 (1999): 9-30.

8. Michael Polanyi, The Tacit Dimension (New York: Doubleday Company, 1966).

9. Jérôme Denis and David Pontille, "Material Ordering and the Care of Things," Science, Technology \& Human Values 40, no. 3 (2015): 338-367; Nicky Gregson, Alan Metcalfe, and Louise Crewe, "Practices of Object Maintenance and Repair: How Consumers Attend to Consumer Objects within the Home," Journal of Consumer Culture 9, no. 2 (2009): 248-272.

10. María Puig de la Bellacasa, Matters of Care, 42-48; see also Bruno Latour, "From Realpolitik to Dingpolitik-An Introduction to Making Things Public," in Making Things Public. Atmospheres of Democracy. Catalogue of the show at ZKM, ed. Bruno Latour and Peter Weibel (Cambridge, MA: MIT Press, 2005), 4-31.

11. Bruno Latour, Aramis, or the Love of Technology. Transl. Catherine Porter (Cambridge, MA, London: Harvard University Press, 1996).

12. Antoine Hennion, "Attachments, You Say? ... How a Concept Collectively Emerges in One Research Group," Journal of Cultural Economy 10, No. 1 (2017): 112-121.

13. Denis and Pontille, "Material Ordering and the Care of Things," 355 and 353.

14. Steven J. Jackson, "Speed, Time, Infrastructure. Temporalities of Breakdown, Maintenance, and Repair," in The Sociology of Speed, ed. Judy Wajcman and Nigel Dodd (Oxford: Oxford University Press, 2017), 169-205, here: 179 and 183. On the interference of temporal regimes of embodied human experience and the materialities of technology, see also the contributions of Nicole Starosielski and Marisa Leavitt Cohn in this volume.

15. Thorsten Metzner, "Hauptstadtflughafen. TÜV hält BER-Terminplan für stark gefährdet," Der Tagesspiegel, April 11, 2019. Available at https:// www.tagesspiegel.de/berlin/hauptstadtflughafen-tuev-haelt-ber-terminplan-fuer-stark-gefaehrdet/24210992.html (accessed June 15, 2019). The TÜV (Technischer Überwachungsverein) Rheinland is a technical inspection association that provides inspection and product certification services according to international standards. The BER indeed opened on October 31, 2020.

16. The calculation is based on an extrapolation from the last reliable data and the assumption of a linear progression of costs. See the website "Flughafen Berlin (BER) Kosten," https://www.flughafen-berlin-kosten.de/ (accessed June 15, 2019).

17. See Michel Callon's analysis of the unsuccessful project to develop an electric vehicle in the 1970s, Michel Callon, "The Sociology of an Actor-Network. The Case of the Electric Vehicle," in Mapping the Dynamics of Science and Technology: Sociology of Science in the Real World, ed. Michael Callon, John Law, and Arie Rip (Basingstoke: Macmillan, 1986), 19-34. 
18. A parliamentary committee of inquiry investigated the mismanagement between 2012 and 2016 and published a report of 1.269 pages in June 2016, Bericht des 1. Untersuchungsausschusses des Abgeordnetenhauses von Berlin - 17. Wahlperiode-zur Aufklärung der Ursachen, Konsequenzen und Verantwortung für die Kosten- und Terminüberschreitungen des im Bau befindlichen Flughafens Berlin Brandenburg Willy Brandt (BER), Drucksache 17/3000, June 14, 2016. Available at https://www.parlament-berlin. de/C1257B55002AD428/vwContentbyKey/206D7oE8863Co6F9C12580C60051E19A/\$FILE/d17-3000.pdf (accessed May 27, 2018).

19. See also Bruno Latour, "Trains of Thought: Piaget, Formalism, and the Fifth Dimension," Common Knowledge 6, no. 3 (1996): 170-191, here: 175; Gabriele Schabacher, "Mobilizing Transport. Media, Actor-Worlds, and Infrastructures," Transfers. International Journal of Mobility Studies 3, no. 1 (Spring 2013): 75-95.

20. "Berlin: 750 Monitore am BER schon veraltet," Spiegel Online, http://www. spiegel.de/wirtschaft/unternehmen/berlin-750-monitore-am-ber-schonveraltet-a-1198359.html (accessed November 8, 2019).

21. “S-Bahn-Lüftungsfahrt zum Airport BER," Berliner Verkehrsblätter 6o, no. 4 (2013): 67.

22. See "Master Plan BER 2040," https://www.berlin-airport.de/de/presse/ publikationen/geschaeftspartner/2018/2018-masterplan-ber-2040.pdf (accessed June 15, 2019).

23. Bruno Latour and Albena Yaneva, "'Give me a gun and I will make all buildings move': An ANT's View of Architecture," in Explorations in Architecture: Teaching, Design, Research, ed. Reto Reiser (Basel: Birkhäuser, 2008), 80-89, here: 86.

24. Stewart Brand, How Buildings Learn: What Happens After They're Built (London: Penguin, 1994), 10, 12.

25. Brand, How Buildings Learn, 13 .

26. Brand, How Buildings Learn, 13 .

27. Sonja Gurris, "Führung auf dem Flughafen BER. Der Airport, über den Berlin lacht," NTV, December 2, 2017. Available at https://www.n-tv.de/reise/DerAirport-ueber-den-Berlin-lacht-article20156588.html (accessed June 15, 2019). Interestingly enough, this experience was not offered on the international site.

28. Reinhold Reith and Georg Stöger, "Einleitung. Reparieren - oder die Lebensdauer der Gebrauchsgüter," Technikgeschichte 79, no. 3 (2012): 173-184.

29. Graham and Thrift, "Out of Order," 9. As Jörg Potthast has shown with regard to the work of air traffic controllers, even the seemingly "normal case" exhibits high degrees of disorder, Jörg Potthast, "Ethnography of a Paper Strip: The Production of Air Safety," Science, Technology \& Innovation Studies 4, no. 1 (2008): 47-68, here: 51f.

30. Sigrid Kannengießer, "Repair Cafés - Reflecting on Materiality and Consumption in Environmental Communication," in Environmental Communication Pedagogy and Practice, ed. Tema Milstein, Mairi Pileggi, and Eric Morgan (London: Routledge, 2017), 183-194. 
31. For a historical perspective, see Daniela K. Rosner and Fred Turner, "Theaters of Alternative Industry: Hobbyist Repair Collectives and the Legacy of the 196os American Counterculture," in Design Thinking Research: Building Innovators, ed. Hasso Plattner, Christoph Meinel, and Larry Leifer (Berlin/ Heidelberg/New York: Springer, 2015), 59-69.

32. Jackson, "Rethinking Repair," 221 and 222.

33. Entry "repair, v.2," in Oxford English Dictionary, Oxford University Press 2019. Available at https://www.oed.com (accessed November 8, 2019).

34. For the media history of accidents and their reconstruction, see Christian Kassung, ed. Die Unordnung der Dinge. Eine Wissens- und Mediengeschichte des Unfalls (Bielefeld: transcript, 2009).

35. For the Bosch company that was contracted to build the fire detection system and safety technology for about 12 Million Euro the contract has multiplied up to 66.4 Million Euro in 2018, because of supplements due to the postponed opening and pricing on hourly basis (Thorsten Metzner, "Die Mängelliste wird kürzer. Wo es jetzt noch hakt auf der BER-Baustelle," Der Tagesspiegel, June 5, 2019. Available at https://www.tagesspiegel.de/ berlin/die-maengelliste-wird-kuerzer-wo-es-jetzt-noch-hakt-auf-der-berbaustelle/24420576.html (accessed June 19, 2019)).

36. See also Gabriele Schabacher, "Abandoned Infrastructures. Technical Networks beyond Nature and Culture," Zeitschrift für Medien- und Kulturforschung 9, no. 1 (2018): 127-145.

37. Denis and Pontille, "Material Ordering and the Care of Things," $35^{2}$.

38. Stephen Cairns and Jane M. Jacobs, Buildings Must Die. A Perverse View of Architecture (Cambridge, MA/London: MIT Press, 2014), 2 and 1.

39. Michael Thompson, Rubbish Theory: The Creation and Destruction of Value (Oxford and others: Oxford University Press, 1977).

40. Cairns and Jacobs, Buildings Must Die, 49, $5^{8 .}$

41. Marisa Leavitt Cohn, "Convivial Decay: Entangled Lifetimes in a Geriatric Infrastructure," CSCW'16. Proceedings of the 19th ACM Conference on Computer-Supported Cooperative Work \& Social Computing 2016: 1511-1523, here: 1511 and 1513 .

42. Cairns and Jacobs, Buildings Must Die, 111 and 103. On obduracy in urban contexts, see Anique Hommels, Unbuilding Cities. Obduracy in Urban Sociotechnical Change (Cambridge, MA/London: MIT Press, 2008). On ruins and ruination, see Gastón R. Gordillo, Rubble. The Afterlife of Destruction (Durham/London: Duke University Press 2014).

43. Cairns and Jacobs, Buildings Must Die, $5^{8}$.

44. Metzner, "Hauptstadtflughafen. TÜV hält BER-Terminplan für stark gefährdet."

45. Paul N. Edwards, "Infrastructure and Modernity," in Modernity and Technology, ed. Thomas J. Misa, Philip Brey, and Andrew Feenberg (Cambridge, MA: MIT Press, 2003), 185-225, here: 185 and 194.

46. Edwards, "Infrastructure and Modernity," 195 and 196.

47. Roland Barthes by Roland Barthes. Transl. Richard Howard (Berkeley/Los Angeles: University of California Press, 1977), 46. 
48. Marianne de Laet and Annemarie Mol, "The Zimbabwe Bush Pump: Mechanics of a Fluid Technology," Social Studies of Science 30, no. 2 (April 2002): 225-263, here: 225 and 226.

49. Jackson, "Speed, Time, Infrastructure," 179.

50. Farman, "Repair and Software."

51. On workarounds see Gabriele Schabacher, "Im Zwischenraum der Lösungen. Reparaturarbeit und Workarounds," in Workarounds. Praktiken des Umwegs, ed. Holger Brohm, Sebastian Gießmann, Gabriele Schabacher, and Sandra Schramke (Berlin: Philo Fine Arts, 2017), xiii-xxviii; on bricolage see Claude Lévi-Strauss, The Savage Mind (London: Weidenfield and Nicolson, 1966), 16f.; on artisan tinkering Simon Schaffer, "Easily Cracked. Scientific Instruments in States of Disrepair," Isis 102, no. 4 (2011): 706-717, here: 708; on improvisation Henke, "The Mechanics of Workplace Order," 66-69.

52. Bruno Latour, "A Cautious Prometheus? A Few Steps Toward a Philosophy of Design (With Special Attention to Peter Sloterdijk)," in: Proceedings of the 2008 Annual International Conference of the Design History SocietyFalmouth, 3-6 September 20o9, ed. Fiona Hackne, Jonathn Glynne and Viv Minto (Boca Raton: Universal Publishers, 2008), 2-10, here: 4 and 5 .

53. Latour, "A Cautious Prometheus?," 4, 11 and 12.

54. Latour, "A Cautious Prometheus?," 5.

55. See Ben Jervis and Alison Kyle, eds. Make-do and Mend. Archaeologies of Compromise, Repair and Reuse (Oxford: Archaeopress, 2012); Paula Jarzabkowski and Trevor Pinch, "Sociomateriality is 'the New Black': Accomplishing Repurposing, Reinscripting and Repairing in Context," M@n@gement 16, no. 5 (2013): 579-592; David Keller and Maria Dillschnitter, eds. Zweckentfremdung. 'Unsachgemäßer' Gebrauch als kulturelle Praxis (Paderborn: Fink, 2016).

56. Edgerton, Shock of the Old, 38, 43 and 209.

57. Maren Mayer-Schwieger, "Umwege auf See. Zur Pflanzenverschiffung Ende des 18. Jahrhunderts," in Workarounds. Praktiken des Umwegs, ed. Holger Brohm, Sebastian Gießmann, Gabriele Schabacher, and Sandra Schramke (Berlin: Philo Fine Arts, 2017), 146-156.

58. On the history and historiography of maintenance, see Russell and Vinsel, "After Innovation, Turn to Maintenance."

59. Jackson, "Rethinking Repair," 231.

6o. Bruno Latour and Emilie Hermant, Paris ville invisible (La Décourverte: Les Empêcheurs de penser en rond, 1998), 26. On the holistic knowledge involved in care practices, see also Douglas Harper, Working Knowledge. Skill and Community in a Small Shop (Chicago: The University of Chicago Press, 1987); Stefan Krebs, “'Dial Gauge versus Senses 1-o'. German Car Mechanics and the Introduction of New Diagnostic Equipment, 1950-1980," Technology and Culture 55, no. 2 (2014): 354-389.

61. Cohn, "Convivial Decay," 1513.

62. In English one can still find this meaning of caring and guarding in the word waiter (entry “waiter, n.," in Oxford English Dictionary (OED). Oxford 
University Press 2019. Available at https://www.oed.com (accessed November 8,2019$)$ ) as well as in older - today obsolete - meanings of the verb wait as "to (keep) watch" (entry "wait, v.1," OED 2019). The verb maintain, in turn, refers not only to the upkeep of things, but also in general to the actions of supporting, assisting, defending, continuing, prosecuting, preserving, and providing for the sustenance, for example, of an animal (entry "maintain, v.," OED 2019). See also Gabriele Schabacher, "Waiting. Cultural Techniques, Media and Infrastructures," in Cultural Techniques. Spaces, Texts, Collectives, ed. Jörg Dünne, Kathrin Fehringer, Kristina Kuhn, and Wolfgang Struck (London/New York: De Gruyter, 2020), 71-84.

63. Entry "warten," in Jakob und Wilhelm Grimm, Deutsches Wörterbuch, vol. XIII (Leipzig: Hirzel, 1922), column 2137.

64. Grimm, Deutsches Wörterbuch, column 2141.

65. In Being and Time Heidegger describes concern as the fundamental way of Being-in-the-world, Martin Heidegger, Being and Time [1962]. Transl. John Macquarrie \& Edward Robinson (Oxford/Cambridge: Blackwell, 2001), ${ }_{5}^{6}$.

66. Martin Heidegger, "Building Dwelling Thinking," in Martin Heidegger, Poetry, Language, Thought. Transl. Albert Hofstadter (New York and others: Harper \& Row, 1971), 145-161, here: 149.

67. Hans P. Hahn, "Das 'zweite Leben' von Mobiltelefonen und Fahrrädern. Temporalität und Nutzungsweisen technischer Objekte in Westafrika," in Kulturen des Reparierens. Dinge - Wissen - Praktiken, ed. Stefan Krebs, Gabriele Schabacher, and Heike Weber (Bielefeld: transcript, 2018), 105-119.

68. Cohn, "Convivial Decay."

69. Daniela K. Rosner and Morgan Ames, "Designing for repair?: Infrastructures and Materialities of Breakdown," CSCW'14. Proceedings of the 17th ACM Conference on Computer Supported Cooperative Work \& Social Computing, Baltimore, Maryland, USA - February 15-19, 2014: 319-331, here: 319.

70. Denis and Pontille, "Material Ordering and the Care of Things," 358.

71. See the respective website "Fairphone / The phone that cares for people and planet," https://www.fairphone.com/en/ (accessed June 20, 2019).

72. John Stark, "Product Lifecycle Management," in Product Lifecycle Management (Volume 1). 21st Century Paradigm for Product Realisation, third ed., ed. John Stark (London and others: Springer, 2015), 1-29.

73. Shozo Takataa et al., "Maintenance: Changing Role in Life Cycle Management," CIRP Annals 53, no. 2 (2004): 643-655, here: 643.

74. Rajkumar Roy et al., "Continuous Maintenance and the Future-Foundations and Technological Challenges," CIRP Annals 65, no. 2 (2016): 667-688, here: 667 .

75. Bernhard Siegert, Cultural Techniques. Grids, Filters, Doors, and Other Articulations of the Real. Transl. Geoffrey Winthrop-Young (New York: Fordham University Press, 2015).

76. See Michael Gamper and Helmut Hühn, eds. Zeit der Darstellung. Ästhetische Eigenzeiten in Kunst, Literatur und Wissenschaft (Hannover: Wehrhahn, 2014). 
77. Brand, How Buildings Learn, 23 .

78. See also Christopher Alexander, The Timeless Way of Building (New York: Oxford University Press, 1979).

79. Denis and Pontille, "Material Ordering and the Care of Things," 359.

8o. de Laet and Mol, "The Zimbabwe Bush Pump," 235 and 226.

81. Latour and Yaneva, "Give me a gun," 80.

\section{About the Author}

Gabriele Schabacher is Professor of Media and Culture Studies at the Johannes Gutenberg University of Mainz, Germany. Her research interests include the media history of traffic, mobility, and infrastructures, the cultural techniques of repair, and the media history of seriality. Recent co-edited volumes focus on the cultures of repair (2018) and on the practices of workarounds (2017). 



\title{
3. Problems of Temporality in the Digital Epoch
}

\author{
Yuk Hui
}

\begin{abstract}
This essay approaches the question of time by considering how digital media have reconstructed the relations between past, present, and future. It proposes that the past and present are becoming more and more determined by future events that have not yet happened but are paradoxically already there. Building on Bernard Stiegler's analysis of primary, secondary, and tertiary retention, it argues that the missing term in current understandings of time is tertiary protention, or the phenomenon of preemption.
\end{abstract}

Keywords: philosophy of technology, preemption, interobjectivity, time, futurity, China

I have chosen a monstrous title, "Problems of Temporality in the Digital Epoch," in that it is difficult if not impossible to treat the subject of temporality in general because every attempt to clarify temporality inevitably becomes lost in confusion, as Saint Augustine once said in his Confessions: "What then is time? If no one asks me, I know what it is. If I wish to explain it to him who asks, I do not know." Every attempt to seize hold of what time is leads to ekstasis, or being outside of itself, which recursively extends to the indefinite. I do not intend to construct an ontology of time here, since it will be doomed to failure; but concerning the monstrosity of the concept of temporality, I also cannot let complacency triumph by taking for granted the present transformation of temporality. Therefore, I would like to talk about problems here. To focus on these problems, I will discuss only individual time and historical time, limiting myself to the question of the future. If I posit the future as a sphere of problems (with the understanding that for

Volmar, A. and K. Stine (eds.), Media Infrastructures and the Politics of Digital Time: Essays on Hardwired Temporalities. Amsterdam: Amsterdam University Press, 2021 DOI 10.5117/9789463727426_CHO3 
certain people the future is always a problem on account of its uncertainty), it is because I would like to suggest a need to strike free from such futures, which is nothing posthuman but too human.

\section{Orders of Temporal Magnitude}

In general, we can approach the question of time in terms of a threefold temporality, namely past, present and future, according to different orders of magnitude. In the first instance, we understand time from an objective geometrical point of view, that is to say, the spatialization into geometrical form divided by second, minute, and so on so forth. For the ancient Greeks, time is thus characterized as "between" (metaxu); for the Stoics, it is "interval" (diastama); and for Augustine, it is sentimus intervalla temporum. In the second case, we approach time in terms of living experience, in which its thickness cannot be reduced to geometrical points but rather must be grasped as indivisible totality, for example, what Henri Bergson calls durée. Lastly, we approach time from the perspective of history, as a past that we have never lived but that belongs to us and remains the condition of our experience. Rather than being merely different points of view, however, these notions of time correspond to different orders of magnitude. ${ }^{1}$ Between these different orders of magnitude, as I argue, we can locate forms of technological mediation that complexify what Heidegger calls the temporalization of past, present and future. In turn, this means that the mediation between these orders of magnitude is subject to an analysis of the evolution of technology.

This brings us to the modes of mediation in the digital epoch. Digital technology has brought a tremendous challenge to the previous understanding of time by reconstructing the relations between these orders of magnitude. For example, we know that with digital technology it is possible to divide a second of sound into milliseconds of microsound, as demonstrated by the granular synthesis of Curtis Roads and Iannis Xenakis. ${ }^{2}$ Such works open a new relation to indivisible human experience, in that a listener is not be able to perceive the millisecond as an individual sound but instead hears only the whole that is already synthesized, as when Leibniz says that when we hear the roar of the ocean, we do not perceive the petites perceptions of the wave but only a synthesis en gros. Nevertheless, in and between these two orders of magnitude, that is to say, in and between the micro and the phenomenological, interobjective relations can be established through digital technology that are available 
to visual display. By interobjective relations I mean those relations that exist independently of associations made by the human mind and that are rather discovered in their constant process of materialization according to new epistemologies and technologies. In On the Existence of Digital Objects, I tried to demonstrate that the history of technology could be considered as a series of discoveries and masteries of interobjective relations (or discursive relations). ${ }^{3}$ In this sense, we can see that temporality is always material; it is hardwired in the technologies that constitute it, and this process of hardwiring will continue indefinitely. Interobjective relations in their prevailing form today - data - are those which reconstitute the dynamic of temporalization.

This brief summary is meant to show that technological evolution alters the question of temporality precisely because it constitutes new interobjective relations that penetrate into different orders of magnitude that have hitherto been unrealized. It is not that technology reconfigures physical time; rather, it brings about new dynamics in what we might call, following Heidegger, the temporalization of past, present, and future. My emphasis, as mentioned, will be on the future. I would like to put my arguments in a simple proposition: our past and present are becoming more and more determined by future events that have not yet happened but are paradoxically already there. The future is a temporality that exceeds what is "hardwired"; however, "being hardwired" remains its most important condition (this is the reason I distinguish discursive relations from existential relations).

The ultimate future for every individual is of course death; the ultimate future of humankind is the solar explosion. But it is also limits that give weight and meaning to temporality instead of pure becoming. Human being anticipates its own death, which is a future already given and is called destiny. Such is Martin Heidegger's famous notion of being-towards-death, in which the philosopher ties finitude to the authenticity [Eigentlichkeit] of human existence, which he calls Dasein. Heidegger's privileging of authenticity consists of two gestures: first, he emphasizes the privation of the They [das Man], a fictional future that separates Dasein from its own time; second, he understands time as self-constituting, by affirming a future that is transcendent, namely death, and refusing a future that blinds Dasein from truth. ${ }^{4}$ However, at stake is the exact nature of this limit. The digital epoch, as I want to argue, is characterized precisely by this fictive futurity, not only because the gap between the world and science fiction is diminishing but also because the future is becoming a metaphysical force and is in the process of liberating itself in all domains. 


\section{The Problem of Tertiary Protention}

Today, technological development, with the combination of big data and predictive algorithms, has rendered what I call tertiary protention more visible and made the relation between the future and the They more explicit than ever. In Husserl's phenomenology of time-consciousness, he distinguishes primary and secondary retentions and protentions. ${ }^{5}$ To assist this with an example, let us consider Johann Strauss's An der schönen blauen Donau. When I listen to the piece for the first time, I retain every now of the melody because every now is always no longer; this is called primary retention. At the same time, I also anticipate the coming melody, without which I would be unable to comprehend the phrasing and would hear not music but only sound; this anticipation of the coming "now," the "not-yet," is primary protention. If tomorrow, I remember the Blauen Donau, it is no longer a temporarily retended "now" but rather a recollection, namely, a memory or secondary retention; and since I already have memory of the music, I am able to anticipate the end of every phrase and the end of the piece as well, as secondary protention. Building on Husserl's concepts of primary and secondary retention, Bernard Stiegler has suggested what he calls tertiary retention, or artificial memories. ${ }^{6}$ For example, my secondary retention of the Strauss piece is not reliable in that it vanishes over time, but a CD may help me to recover that memory. The gramophone (analogue) and the $\mathrm{CD}$ or now $\mathrm{MP}_{3}$ (digital) are tertiary retentions that in some way invoke our primary and secondary retentions and protentions like Proust's madeleine. I propose to add to this schema a tertiary protention, a form of anticipation that is no longer my own subjective projection but rather a projection that is imposed on me and proceeds ahead of me. Protention cannot be reduced to any form of retention or any combination of retentions because protention is ontologically different from retention. Otherwise, there would be no différance, in the sense of Derrida, because différance is the "retention and protention of differences." I argue that tertiary protention is the missing term necessary to bring forward a full hermeneutic circle and analysis of the contemporary politics around attention, desire, and capital.

Everyone using social media knows very well that we are dealing with recommendations all the time, as if the future was always already present in the form of multiple choices. A typical example used for decades by computer scientists working in the field of ubiquitous computing is the following: you just finished work and are feeling tired while driving home, and when you arrive, you find a cup of warm, freshly brewed coffee waiting for you, with exactly the type of coffee bean you have in mind. This example 
is only the tip of the iceberg of the increasing power and phenomenon of tertiary protention, described by many as preemption. Through preemptive algorithms, we are witnessing a mode of marketing based no longer only on psychoanalysis, as what Edward Bernays, the father of public relations and nephew of Sigmund Freud, used, but rather on detailed statistical study of behaviors in order to capture the future through the past and impose it on the present. Here, we observe the repetition of the future, functioning as a new synthesis apart from those Gilles Deleuze analyzed in Difference and Repetition. ${ }^{8}$ Deleuze discusses three syntheses of time: the Humean repetition of habits, the Freudian return of trauma, and the Nietzschean eternal recurrence of the same in which the future is already at the present. In this fourth synthesis of time that we call tertiary protention, the future is also at the present, but it does not carry the same sense as Deleuze's third synthesis; in fact, it is a return to a mode of repetition that is complex but mechanical and homogenous. In our digital milieu consisting of smart objects, smart homes, smart cities, and social networks, the time of each individual is fragmented and reorganized so that tertiary protention is ahead of us.

What could have been called an insight decades ago is today no more than common sense in view of the becoming of technical systems. (I take the term "system" here in the sense of Jacques Ellul, who in the 1970s already clearly described the totalization and autonomy of a technical system in view of the computer's capacity for data processing. ${ }^{9}$ ) In On the Existence of Digital Objects, I suggest to liberate time from the increasing threat of the determination of tertiary protention by inventing new temporal organizations through creation of alternative technological architectures and algorithms. ${ }^{10}$ This involves fundamentally a reorganization of interobjective relations. For example, from 2011 to 2012, I worked with the computer scientist Harry Halpin to develop a prototype of a social network based on groups instead of individuals, with each group being based on a common project and each project referring also to a projection, or an investment of time. ${ }^{11}$ Additionally, in 2013, I worked with a group of computer scientists from Princeton University and Deutsche Telekom Laboratory to propose a new recommender system based on groups. If the new form of interobjective relations has already acted upon us in our daily lives and constitutes what Antoinette Rouvroy calls "data behaviourism," then it extends much further than individual time, constituting instead a collective imagination of the future. ${ }^{12}$ It is because of this futurality powered by the algorithmic processing of data that I would like to proceed to the second problem concerning our collective past and its becoming, which we call world history. 


\section{The Problem of the Digital Future}

It is not only "personal time" that is hardwired but also historical time. Through the hardwired geometrical time that we call synchronization, we arrive at a historical time that we call globalization. It is in the axis of time in which the historical temporalities of all cultures converge that we can articulate a world history. The danger of synchronization is that with digital technology, especially the data economy, we are all moving towards one future, the pinnacle of humanism. This future is associated with technological singularity, artificial intelligence, machine learning, and the realization of the homo deus. Yuval Noah Harari, the author of Homo Deus, embraces a human-algorithm reduction, proposing in the name of "life science" the following:

1. Organisms are algorithms, and humans are not individuals - they are 'dividuals,' i.e. humans are an assemblage of many different algorithms lacking a single inner voice or a single self.

2. The algorithms constituting a human are not free. They are shaped by genes and environmental pressures, and take decisions either deterministically or randomly — but not freely.

3. It follows that an external algorithm could theoretically know me much better than I can ever know myself.... Once developed, such an algorithm could replace the voter, the customer and the beholder. Then the algorithm will know best, the algorithm will always be right, and beauty will be in the calculations of the algorithm. ${ }^{13}$

The transhumanist tone, claiming insight from "life science," has already pointed to a future of humanity that can be reduced to an artificial intelligence governed by a super intelligence, which knows anything and everything. This is a future that will arrive at us. Questioning the "we" is delicate in that we may easily fall into one of two sides. One is ethnocentrism and ethnofuturism, which often fall prey to proto-fascism. The other is a universalism that affirms the realization of humanity through technical advancement. But it is because of the delicacy of this question that I think we cannot avoid it in our reflection on the digital future or futures, lest we lose sight of the issue of locality. To tackle this question, we will need to reassess the history of technology and challenge the universalist conception of it. I use the term "universalist" to refer to the unreflective belief that there is only one type of technology, no matter if it is Chinese, Indian, Amazonian, or European, with differences among these contexts being ascribed to levels of advancement. Nevertheless, it is by the advancement of technology that the future merits its name. 
Therefore, I will go a step further to suggest that we must liberate ourselves from this deterministic view of history in the digital epoch, called forth by transhumanist futurisms and prometheanisms, such as accelerationism. The problem is not that the transhumanist's proposal of immortality or a-mortality problematizes the "being-towards-death" of Heidegger; rather, the realization of the homo deus, or technological singularity, as end of history expresses the desire and the delirium of the modern. This competition for technological singularity will characterize the geopolitics of the coming decade. Technological competition, which historically took shape largely as military competition, has now become a domestic competition in terms of automated technologies, artificial intelligence, deep learning, nanotechnologies, and genetic technologies that define the sovereignty of the nation state. China released a whitepaper in August 2017 that states its wish to become the leading country in AI by 2030, with AI being introduced into primary school education and onward in the meantime. In September 2017, Russian President Vladimir Putin gave a speech to the Russian children in which he proposed that "whoever leads in AI will lead the world." Earlier, during his 2016 US presidential campaign, Donald Trump proposed to bring back jobs that China had "stolen," which could only be done, as some economists suggested, by implementing automation on a large scale, so that the competition would be between Chinese robots and American robots. The geopolitics of the coming decades could be understood as a competition towards singularity under names such as sinofuturism and Asian futurism.

It is within the desire for technological singularity, which conceals the ultimate dream of all forms of prometheanism, that we observe histories converging further on the same axis of time, moving towards a single direction and the realization of the homo deus. Coincidently, this convergence resonates with the progress of history as theodicy in the sense of Leibniz, as Hegel famously claimed. Digital studies must not relinquish this question too quickly and analyze only phenomena produced by new industrial technologies. Instead, it is necessary to open new directions for the development of digital technology in order to imagine a future with technical diversities.

As with the first problem, in which the future is already determined through calculation, which commands $\tau \dot{\alpha} \pi \dot{\alpha} \nu \tau \alpha$ like the lightening of Heraclitus, time in the second problem is constrained by a world history presented as a single time axis, in which the present is always already determined by a future imposed as necessity. World history, in this understanding, follows a defined order: premodern, modern, postmodern, 
apocalypse. I would like to argue that modernization is a synchronization based on a universalist concept of technology that confuses universalism with universalization, with the latter being inseparable from colonization qua technological globalization. We are confronted with a homogenous technological future, which nurtures fanaticism as well as the fanaticism of speculation. This unquestioned universalism of technology must be exposed to its limit so that we can imagine a plurality of technological futures and therefore reopen the question of technology and hence imagine a new geopolitics and a new globalization to come.

\section{Antinomy of the Universality of Technology}

Conceiving the possibility of techno-diversity, I suggest, is possible only by breaking away from the synchronization described above to envisage different technological futures. This, in turn, is possible only if we recognize the plurality of cosmotechnics, a term that I coined to distance us from the impression that technology is universal. Here is a primary definition of cosmotechnics: the unification of the cosmic and moral orders through technical activities. This conceptualization is based on an antinomy of the universality of technology addressed in my recent book, The Question Concerning Technology in China: An Essay in Cosmotechnics, which could be stated:

Thesis: technology is an anthropological universal, understood as the exteriorization of memory and liberation of bodily organs, as some anthropologists and philosophers of technology have formulated.

Antithesis: technology is not anthropologically universal, being enabled and constrained by particular cosmologies, which go beyond functionalities and utilities. ${ }^{14}$

The peculiarity of the Kantian antinomy is that each thesis holds on its own but opposes the other; such an antinomy must be resolved by a form of thinking beyond universality and particularity. Synchronization relies on the thesis and undermines the antithesis. To answer the question concerning futurity, we must clarify the antithesis before a resolution can emerge. This is why I propose that each culture should develop its own history of cosmotechnics by systematically rediscovering and formulating its epistemologies and tracing the history of its epistemes in response to our current historical moment. The aim of conceptualizing cosmotechnics 
is to reopen the question of technics that was unfortunately closed down in past centuries.

Following the analysis in Heidegger's 1949 lecture later published as "Die Frage nach der Technik," we find two concepts and essences of technics. ${ }^{15}$ The first is the Greek techne, which means poiesis, or bringing forth (Hervorbringen), and the second is modern technology, the essence of which is no longer the Greek technē but rather enframing (Gestell), meaning that everything is considered calculable and exploitable as resources (Bestand). Gestell stands as the symbol of the modern, in which cosmologies are replaced with astrophysics. However, this discourse of technology is fundamentally European. While it is difficult, if not impossible, to position other kinds of technics - for example, the Chinese, Indian, or Amazonian — without reducing them to Greek technē, it is self-evident that they are not "modern" technologies.

Therefore, we need a new way of understanding technologies outside of Europe because they are neither technē nor modern technology. It is intriguing to hear non-European philosophers, when explain the origin of technologies in their cultures, refer immediately to the mythology of Prometheus, as if the Greek technē were the origin of all technics. This reference is symptomatic in the sense that it means a disorientation, in the double sense of the word. Historians of technology often compare the advancement of a particular technology in different cultures, for example, papermaking in China and Europe, or the dynamic of technical systems in China and Europe, as the French historian Bertrand Gilles has done. However, it is also possible that these comparisons easily circumvent the antinomy that we have raised above, namely the cosmological and metaphysical specificity of cosmotechnics. The question of technological futures must be approached through historical and metaphysical investigations of cosmotechnics - a task I have suggested carrying out by reconstructing a Chinese technological thought.

I attempted to do so by tracing the relation between dao (道) and $q i$ ( 器) in Confucianism, Neo-Confucianism and New Confucianism through a characterization of the dynamics between these two metaphysical categories as different epistemes. Dao literally means path, and $q i$, utensils (not to be confused with the $q i$ or ch'i [氣] usually translated as energy). Dao is a moral cosmological thinking that situates humans as cosmological beings and leads them toward the good. The unification of $q i$ and dao is neither a formula nor a static being; rather, it is dynamic and corresponds to the episteme of the epoch. I won't be able to elaborate on this development. And if I have attempted to diversify the concept of technics, it is because 
I want to put forward the following questions: Is it possible to take this historical moment to rethink digital technology, not only to understand its transformative power in new social phenomena but also to open up the question of technology and technodiversity? Is it possible to nourish digital technologies with new epistemologies and epistemes in light of the concept cosmotechnics?

Let us return to the two problems of temporality that we have aimed to sketch out in this paper. We see how digital technology interacts with other orders of temporal magnitude and have attempted to problematize these observations. I emphasize problems because these are what allow us to invent. The future already contained in preemptive algorithms is a battlefield for the studies of ethics and technology; to engage with the question posed by this future, I think it is important to unfold the epistemological and ontological presuppositions of these algorithms in order to put forward alternatives. We have also challenged the universalist concept of technology and proposed that in order to break from such technological determinism, it is necessary to reopen the question of technodiversity, which is also the condition of noodiversity. Therefore, we must be critical with notions of the future of humanity presented as the realization of homo deus or the progress of modernity. By rejecting the linear path attached to the image of technological progress, we also reject the politics of acceleration as the only option available for resolving social and political problems. We seek to allow multiple futures to emerge and remain irreducible to the global axis of time perceived as world history since what is called modernity.

\section{Notes}

1. See Yuk Hui, On the Existence of Digital Objects (Minneapolis: University of Minnesota Press, 2015), 29-32.

2. See Curtis Roads, Microsound (Cambridge, MA: MIT Press, 2004).

3. For more on interobjective relations, see my chapter "The Time of Technical Systems," in On the Existence of Digital Objects, 151-186.

4. See Martin Heidegger, Being and Time, trans. John Macquarrie and Edward Robinson (New York: Harper \& Row, 1962).

5. $\quad$ Edmund Husserl, The Phenomenology of Internal Time Consciousness, trans. J. S. Churchill (Bloomington: Indiana University Press, 2019).

6. See Stiegler, Technics and Time, 2: Disorientation, trans. Stephen Barker (Stanford, CA: Stanford University Press, 2009).

7. Jacques Derrida, "Différance," in Margins of Philosophy, trans. Alan Bass (Chicago: University of Chicago Press, 1982), 15. 
8. Gilles Deleuze, Difference and Repetition, trans. Paul Patton (New York: Columbia University Press, 1994).

9. See Jacques Ellul, The Technological System, trans. Joachim Neugroschel (Eugene, OR: Wipf \& Stock, 2018).

10. See "Logic and Time," in On the Existence of Technical Objects, 221-252.

11. For more, see Yuk Hui and Harry Halpin, "Collective Individuation: The Future of the Social Web," in Unlike Us Reader: Social Media Monopolies and Their Alternatives, ed. Geert Lovink and Miriam Rasch (Amsterdam: Institute of Network Cultures, 2013), 103-116.

12. Antoinette Rouvroy, "The End(s) of Critique: Data Behaviourism versus Due Process," in Privacy, Due Process and the Computational Turn (London: Routledge, 2013), 157-182.

13. Yuval Noah Harari, Homo Deus: A Brief History of Tomorrow (London: Vintage, 2016).

14. Yuk Hui, The Question Concerning Technology in China: An Essay in Cosmotechnics (Cambridge, MA, and London: MIT Press, 2019).

15. Martin Heidegger, "The Question Concerning Technology," in The Question Concerning Technology and Other Essays, trans. William Lovitt (New York: Garland, 1977), 3-35.

\section{About the Author}

Yuk Hui is the author of On the Existence of Technical Objects (Minnesota, 2016), The Question Concerning Technology in China: An Essay on Cosmotechnics (MIT, 2017), and Recursivity and Contingency (Rowman \& Littlefield, 2019). 



\title{
4. Suspending the "Time Domain": Technological Tempor(e)alities of Media Infrastructures
}

\author{
Wolfgang Ernst
}

\begin{abstract}
Any media event is a time function of signals. In favor of a diagrammatic definition of technological media, media archaeological investigation is not only concerned with their structural "hardwired" level but with their operative unfolding-in-time as well. Such an understanding of techno-temporalities does not focus on phenomenal effects of media on humans but primarily refers to the microregimes within technological devices. In that sense, "hardwired temporality" refers to the infrastructuring of time by technologies and to temporal structures which are revealed from within techno-logical knowledge itself. From that arises an epistemology of technical processuality beyond the conventional notion of "time."
\end{abstract}

Keywords: Media archaeology, techno-temporality, clocking, synchronization, UNIX-time, time of non-reality

\section{The Nonhuman Temporality of Technological Knowledge}

In favor of a diagrammatic definition of technological media, media archaeology is concerned not only with their "hardwired" structure but also with their operative unfolding-in-time. This vector places the field close to signal analysis. Any media event is a time function of signals. ${ }^{1}$ In addition, the radical media archaeology of the present media-infrastructural condition requires analysis of its mathematical, algorithmic operations.

Such an understanding of techno-temporalities does not focus on phenomenal effects of media on humans but primarily refers to the microregimes

Volmar, A. and K. Stine (eds.), Media Infrastructures and the Politics of Digital Time: Essays on Hardwired Temporalities. Amsterdam: Amsterdam University Press, 2021 DOI 10.5117/9789463727426_CHO4 
within technological devices. Complementary to discourse analysis, it listens to the implicit epistemic articulations and enunciations of infra-technical operations. In that sense, "hardwired temporality" refers to the infrastructuring of time by technologies and to temporal structures revealed from within techno-logical knowledge itself. From that arises an epistemology of technical processuality beyond the conventional notion of "time."

\section{Clocking as the Anonymous Time Base of Modernity}

The time base of present information society (for which actor-network theory admits nonhuman members) turns out to be a critical focus of analysis. High-frequency trading as data exchange, in the electronic stock market as well as in the communication sphere of the internet, surprisingly recalls late medieval monasteries. A true media archaeology of technically temporalized infrastructures starts here and not in the short memory of the recent present. Benedictine monks who needed periodically exact clocking for prayers according to their monastic rules invented the escapement-driven wheeled clock. Regular oscillation, subdividing movement into equal quanta, is a precondition not only for industrial production but also "social media" communication. Within the von Neuman architecture of current computers, the heartbeat of the time base enables exact synchronization of cycling units in data processing.

The escapement-driven clock, providing occidental culture with its equidistant beats, oscillations, and synchronisms, was invented anonymously. ${ }^{2}$ Such temporality cannot be reduced to the timeline of cultural history but rather finds "itself." There is a rather nonhuman temporality at work in the infrastructuring of techno-logical knowledge.

According to Martin Heidegger, long before computing in the strict sense, information society already started with the modern Weltbild: with the measuring and numerical (scientific) approach to physical nature. ${ }^{3}$ An archaeology of the contemporary therefore starts with what in German is appropriately called "Neuzeit," or modern time, and in a double sense, because the new epoch starts with the mechanical clock itself. Marshall McLuhan, in media-epistemological terms, recedes even further below the clock technology, to the "technologizing" of the spoken word by the ancient Greek vocal alphabet itself. ${ }^{4}$ It was alphabetic writing that cognitively induced the analysis and synthesis of oral speech flow into discrete, digital units. Only when the letter (reduced to the minimum as a binary information unit) becomes radically meaningless in itself does it enable its full range of 
storage, transfer, and symbol manipulation (processing) of meaning. Once more, it is epistemologically remarkable that there is no way to inscribe this cultural technique on the historic timeline because there is neither a precise date nor a known inventor. ${ }^{5}$ Technological culture takes place in anonymous temporality.

\section{Enduring Oscillations versus Transient Phenomena}

Both the pulled string of an instrument and articulated speech, by their very fading out, reveal the endlichkeit (the temporal limit) of any physical signal event. The moment a string is pulled or a sound is articulated, like a breaking wave, the chord already anticipates and senses its very end, almost instantaneously but strictly temporally. A string of an instrument, once struck, never simply articulates the pure sine tone of its proper frequency but carries with it a fading memory of its violently abrupt, sudden, transient start. In the real — that is, physical — world, the picked string fades out due to mechanical loss of energy (Heidegger's "being-toward-death" in its mechanical sense), like a swinging pendulum, a reverberation in the sonosphere.

Any pleasure of a musical performance by real humans in real time and space (be it oral poetry as sung by Homer or a musical concert) is always already accompanied by a melancholic anticipation (that is, irony) of its ending. On the contrary, audiovisual machine recordings, such as gramophone discs and videotapes, can be replayed with no internal sense of ending. To modify Walter Benjamin's analysis on "The Work of Art in the Age of Its Technological Reproducibility," with technical re-petition, the temporal aura (which is based on the allegorical awareness of ending) is lost. ${ }^{6}$ But the reentry of temporality happens within the very signal processing of technological media.

Even electromagnetic waves, such as those calculated by James Clerk Maxwell, propagate very fast but not instantaneously. In 1879 Hermann von Helmholtz initiated a prize by the Berlin Academy of Sciences to answer whether, following Maxwell's mathematical equations, light is in fact part of the electromagnetic wave spectrum and thereby subject to temporality, the literal "speed of light" limit. Radio waves, as turned out by Heinrich Hertz' experiments on the very media-archaeological level (that is, before becoming part of a mass-medium called "radio"), have a sense of ending on their very electrophysical-event level; the secondary level is the modulation of such principally timeless carrier waves by the proper radio program, which by 
its very program structure introduces a symbolical order of beginning and ending by arbitrary cultural and media-economical decisions.

The out-fading oscillations in early spark-driven telegraphy become transformed into stabilized, equi-dynamic, in principle, "eternal," oscillations only by the electronic vacuum tube in feedback circuits. Electronics turned electric signals from ephemeral into regenerative, Bergsonian duration. Only this allowed for the radio transmission of carrier-wave-modulated speech and music, resulting in the pulse modulated digital transmission.

\section{The Non-Sense of "Time" for Technological Analysis}

Technological media know processual realities but not "time"; therefore, the neographism "tempor(e)alities" is preferred in the subsequent argument, which unfolds such media tempor(e)alities on three levels: first of all, within technologies, when the micro-timing of signal transduction is crucial for the event to succeed at all; next, in the human sense of temporal experience, when media events induce phenomenal affects and irritations; finally, "deep" media temporality (logic and machine), which turns out to be rather autonomous from the cultural imaginary of imaginary time a.k.a. "history."

In that context, the very notion of hardwired temporality is a relief. Its ambition is not deep philosophical questioning of the nature of time; temporality is rather a term that names a couple of signal functions, such as the Delta-t, and functions of signal processualities, such as transfer, delay, and even storage. For the close analysis of contemporary media technologies, the arché is not in "time," but a cooriginality where "time" is suspended. Media archaeology is "radical" in the sense that it looks for roots not in "time" but in the technological event. There can be no infrastructuring of temporal orders, only infrastructures triggering notions of "time" as secondary effect. Here, the notion of time as a priori condition of perception (as defined by Immanuel Kant) does not count. If time counts here, it is in the precise sense of clocking which generates what Heidegger denounced as "vulgar" technical time. In that sense, technical infrastructures are not "time" based, but "time-basing." What has been decisive for digital computing in the individual machine counts more interconnected computers. Communication is not only the material infrastructures of cables, antennas, and data-processing devices but also events temporally infra-structured. Synchronization of internet traffic creates an artefactual tempor(e)ality, just as the introduction of a synchronizing master clock was the condition of a universal "time" in the nineteenth century. The master clock, in networked computing and 
communication, has imploded into a myriad of local temporal agencies. This temporal pattern is multiple and invites the replacement of transcendent signifiers with a plurality of more precise termini technici, practicing a language of analysis that bypasses outdated notions like "time" in the mediasphere.

Walter Benjamin defined the nineteenth century as the antiquity of the "now." Indeed, the "present" can already be seen in 1839 when Karl August Steinheil designed a timekeeping system for the synchronization of electromagnetic external clocks by a mechanical central clock whose pendulum triggers alternating positive and negative poled "time impulses" - an interlacing of timekeeping and telegraphy. Today, the radio time signal transmitter DCF $77(77,6 \mathrm{kHz})$ at Mainflingen synchronizes radio clocks wirelessly.

In a radically media-archaeological understanding, techno-logical infrastructures extend from material (technical) to mathematical (logical) grids, to the algorithms embedded in ubiquitous microchips. The imaginary unifying time axis is decomposed into a symbolic concatenation of programmed processes, patterned and interrupted by moments of the tempoReal. Aristotle was radically media-archaeological in book IV of his Physics, defining "time" as resulting from measuring movement sequentially by numbers. Even etymologically, only from incisive cuts does the notion of a temporal order arise.

\section{Synchronization and Functional Timing}

Rigid analysis of technology does not know metaphysical "time" but rather an ensemble of temporal operators. There is no given preexisting "time" but rather an enforced timing, as expressed in the very constructive term of synchronization.

Edmund Husserl once phenomenologically described the mechanism of the inner sense of subjective time. ${ }^{7}$ Such a temporal horizon unfolding as extended present between re- and protention is radically "grounded" and de-metaphysicized when it comes to technological ensembles. Lewis Fry Richardson's once designed a (human) computing "forecast-factory" for real-time calculation of weather, connected to local weather stations in telegraphic "instantaneity." A central official maintains "a uniform speed of progress" for the individual partial calculations, "like the conductor of an orchestra in which the instruments are slide-rules and calculating machines." Panoptically, "he turns a beam of rosy light upon any region 
that is running ahead of the rest, and a beam of clue light upon those who are behindhand." ${ }^{8}$

In the meantime, human synchronization of human "computers" has been replaced by the cybernetic diagram of feedback circuits, replacing the central time control agency by a flexible automation where human monitoring itself becomes integrated into the circuitry. For a moment, technological utopia has become reality: the "Opsroom" for monitoring and control in Stafford Beer's Cybersyn computing structure in Salvador Allende's Chile 1971-73 for national feedback and control of economic data from state-owned factories. The data flow was based on a "Cybernet" teletype network, to be calculated by a central mainframe IBM 360 computer, Cyberstride, and monitored by a collective of humans for positive or negative feedback. Human wetware is wired into the symbolic infrastructure for the option to interrupt and correct: suspending the time series generated by data. ${ }^{9}$ The melodic re- and protentional "inner time consciousness" of the human individual (Husserl) is replaced by the radically "discrete" temporality of counting.

\section{The Emancipation of Technical Timing from Natural "Time"}

Media culture is not shaped by a transcendent timeline, but technologies themselves "shape" time. ${ }^{10}$ In the 1930s, the crystal quartz clock emancipated the time base of technology from the astronomical "natural" reference-an epistemological breaking point. Since 1971, the 555 timer chip has been the most popular integrated circuit for its ability to generate a steady oscillation independent of supply voltage.

Against phenomenological suggestions of endurance, a truly archaeological analysis of temporality is time-discrete. This imperative conditions the rhythmic bias of digital culture. As once expressed by Alan Turing himself, clocking is still the heartbeat of structuring data processing from within. ${ }^{11}$

Against its meaning at first sight, "real-time" data processing is not about being close in time but its actual negation. It replaces the familiar "live" signal transmission by a re- and protentional microtime window of the present; telepresence (Heideggerian "Ent-fernung") becomes predictive coding, a mathematical in-between of humans and their physical environment.

Since 1970, Unix time has described time for operating systems and file formats in terms of the number of seconds that have elapsed since oo:00:00 Coordinated Universal Time (UTC), Thursday, January 1, 1970. This starting date is appropriately called "The Epoch," and thus the epoch of the computational present starts here. Unix subdivides the day exactly into 
86,400 seconds. But this chronotechnical regime does not itself tolerate the interpolation of leap seconds according to atomic clock-generated timekeeping, where a second must be added to the UTC system to coordinate with the variability of the Earth's rotation. Occasionally, when computers have been forced to use the leap-second friendly UTC, such ruptures of the tempoReal have crashed websites and confused airline departures. ${ }^{12}$ Similarly, the Millennium Bug was a reminder at the end of the 199os that time in digital computers is logical and mathematical, not intuitive ("Bergsonian") time.

The tempor(e)ality of "online" timing actually escapes the historiographical timeline. Accurate timekeeping systems such as the cesium atomic clock embedded in GPS signal traffic are rather independent. In computing science, so-called "real-time," or the physical clock measuring physical time as hardware, differs from the logical clock as software; this causes the necessity to synchronize, in intervals, the real-time clock in computers with external time, by requesting time from time servers and then, by intelligent algorithms, equaling the time delay in the Network Time Protocol, based on the Internet Protocol and time synchronization software.

In October 1998, the Swiss watchmaker Swatch announced "Internet Time," which undoes the familiar differential time zones. Every day is divided into 1,00o "beats," creating a new meridian in Biel, home of Swatch itself: the Biel Mean Time (BMT) as universal reference to Internet Time. ${ }^{13}$ This is "an indifferent time, no longer the vectorial time of chronology." ${ }^{14}$ At that point, the familiar historic timeline graph fails.

\section{The Fuzzy Present of Internet Communication}

Any media archaeological analysis of technological communication infrastructures takes their increasingly time-critical nature into account. If "time" is not understood in a unifying metaphysical sense but in its Aristotelian definition, it turns out as a functional plurality of signifying incisions and intervals. What was once electromagnetic "live" radio or TV signal transmission in telecommunication has been replaced by "real time" computation which dissimulates the belatedness of complex calculations of discrete pulse trains so as to trap human perception of the "present." In digital online communication, there is no transmission in time but a timing of data packets (datagrams) that are numerically time-stamped to avoid internet traffic congestion.

In 1972, Bob Metcalfe developed a program called PING for testing the interoperability of networked devices on what would come to be called 
Ethernet. The program would open a connection through the physical and logical network topology to test whether the addressee actually reacts. From that technical implementation of a time-critical test, Vint Cerf developed the Transmission Control Protocol for the ARPANET in 1975, which preceded the actual Internet. So-called time-to-live and ping-to-death are articulations of internet temporality. The past is not "imperfect" anymore but becomes "historical perfect," residually enduring within the present. The "residual" is still active, "not at all as an element of the past, but as an effective element of the present." ${ }^{\prime 5}$

Metaphors like "streaming media" are misleading in their suggestion of a temporal flow. Even with respect to the signal carrier (the "flow" of electricity), information depends on the digitally coded electrons. Where humans believe themselves to be communicating messages via digital channels, a nonhuman temporality is at work between computers. Any political criticism of the microphysics of power has to focus on time-critical events on the most physical level of the OSI network model.

Network culture is less about modernist clock time than about latencies. The delayed present stems from the "hyperbolic temporalities of digitality."16 Speculative media theory asks experimental questions: What if data packets were humans, how (if at all) do they experience time? In Web 2.o packet switching, before any kind of "social memory" is triggered, intermediary storage is a decisive and integral part of the technical transmission itself. The age-old contradiction between archive and transmission collapses in the delayed present. Before there can be any moments of short-time virtual communities ("crowds," or even societies), data networks consist of distributed sparks of ultra-short retentions and protentions.

\section{Time-to-Live and Ping-to-Death}

In the times of internet protocols McLuhan's thesis that the pace of electronic media changes the patterns of temporal perception deserves a closer reading. Time-critical processes take place in the most media-archaeological sense, that is, on the basic layer of bit transfer in the internet, the physical layer. This layer represents the interface between symbolic transfer and material channel of communication and thus embodies very concretely the interlacing of logi(sti)cs and matter which is already implied in the term "technology." It is on this layer that the voltage level of what is meant to represent a logic "zero" and a logic "one" is being defined. The function of this bit-transfer layer is to transform signals from the physical channel into information to 
be passed on to level two of the OSI system. ${ }^{17}$ This identification of signals happens within the time-critical field, such as signal frequency and signal duration, synchronous or asynchronous clocking, and the decision on serial or parallel data transfer.

In communication networks, topological systems are being appropriately expressed by hypertextual links, whereas time-critical processes rarely become apparent. The answer to this is to find a new term that does not nominate a new medium but declares the temporal mode of a mode its decisive media-theoretical criterium. "The real-time web is a set of technologies and practices which enable users to receive information as soon as it is published ... rather than requiring that they or their software check a source periodically for updates." ${ }^{18}$ The communicative practice of instant messaging belongs to this temporal field; in McLuhan's sense, the message of the medium here is immediacy serving to create the illusion of a pseudo-copresence. This recent form of web economy is being defined by communication within the time-critical realm; cyberspace as what Ted Nelsen calls "docuverse" is being replaced by the extreme speeding up of information processing in cybertime. ${ }^{19}$ The internet thus turns out not to be just a topological extension of a generalized archive, but equally as a chrono-technical expression of time.

The time-critical message of the internet requires a close look at the time-critical operations on its physical and logistical levels, such as seen in the "Ping" signal. Each data packet into which a document has been sliced is being observed individually; its transfer happens independent of its preceding or successive packets. This procedure is radically time-critical because it takes place within the so-called "time to live" field that defines the maximal temporal duration in seconds that an IP packet is allowed to exist in the internet. A counter is progressively reduced during this routing; if the TTL-counter reaches zero before the packet has reached its destination, it is annihilated. ${ }^{20}$ Media time is not endless. In TCP/IP as fundamental network program, techniques of synchronization meet a deadly economy of time. "Time to live" means that each data packet is assigned a given lifespan; "time to die" thus becomes a crucial signature of the information age.

Whereas Husserl's phenomenology expresses neuro-cognitive perception of the present in "time diagrams" between retention and protention, media archaeology tries to precisely identify the rather different operations of micro-technical signal transduction and algorithmic data processing. Humans and machines are different in their operative signal processing, resulting in different tempor(e)alities. 


\section{(A)Temporalizing Radio}

The present can be analyzed only when it starts to recede into the past. The concept of a prehistory of the present technological condition refers not only to a "before" in its temporally sequential, historical sense but also to its technological preconditions. This prestructuring "before" endures or re(oc)curs in the present in nonlinear modes. Media archaeological analysis, besides its apparent meaning in a search for "origins," refers to a structural argument: the arché which is the insisting, essential features of a technological system. Heuristically, this means analytic reduction to the essential functions, the elementary bits, a rarefication against discursive redundancies.

All of a sudden, the "recent" is not past but a concealed retreat, the hidden, still copresent ground behind the apparent visible. The technological conditions take place in intervals (epoché) where an established infrastructure remains valid across all apparent political, historical, and cultural changes, just as analog AM radio has endured for more than 80 years almost technologically unchanged. Such intervals, as Delta-t, endure anachronistically (even achronically) when compared with the historical timeline. Public radio, in Germany, dates back to October 1923. As an independent media format, based in autonomous technological implementation, it apparently dies these years in its familiar AM / FM analog technology. A historic "timeline" representation of the heroic radio age is misleading. There is a reentry of "radio" into mobile communication, not as a program format and "broadcasting," but in its purest form as technical medium: wireless (German "Funk") electro-magnetic waves, this time digitally modulated, as in mobile telephony and WLAN internet access. In present mobile communication, there is more radio than ever, even if dissimulated as a condition of possibility.

The infrastructure of wired and wireless networks increasingly becomes interlaced. "Rather than wireless cities of wireless networks, it might be more accurate to speak of the rewiring of cities through the highly reconfigurable paths of chipsets. ${ }^{21}$ In wireless communication, the infrastructure has become mobile itself. McLuhan described the wireless transmission of analog signals as a sphere as "acoustic space" because its inherent message (its implicit "sonicity") has been the wave as temporal form. "Electric speed is approximately the speed of light, and this constitutes an information environment that has basically an acoustic structure." ${ }^{22}$ In digital communication, this almost Heideggerian "Being" of electromagnetic spacehas been inverted from melodic tuning to pulsed rhythm. ${ }^{23}$ Eleni Ikoniadou's 
The Rhythmic Event conceptualizes digital events not as binary sequences of zeros and ones but instead as relational pulsation. ${ }^{24}$ This correlates with the notion of "algorhythmics" - the rhythmic processes of computational algorithms. ${ }^{25}$ In that sense, hardwired temporalities can never be reduced to (infra-)structures, but in reverse, they temporalize such structuring conditions (l'archive, in the sense given by Foucault) themselves, "posting" infrastructures. ${ }^{26}$

While data-processing microchips are still hardwired infrastructures in themselves, the wireless signal transfer has become a dynamic infrastructure consisting of extremely volatile temporal objects: multiple radio-frequency waves transduced by devices using digital signal processing (DSP) embedded within mobile phones and other wireless communication devices. To exhaust the available channels for signal transmission in parallel (known from George Antheil and Hedy Lamarr's invention of "frequency hopping"), "the designers of contemporary wireless DSP chipsets usually supply a palette of different hardwired algorithms alongside generic processors. ${ }^{27}$ Just as in integrated circuits, the integration of hardwired and wireless networks allows for algorithms to operate. Radical mathematization transforms conventional communication engineering into intelligence in its double meaning. ${ }^{28}$ While most of the physical layer of information networks "is quasi-hardwired into semiconductor chips, ${ }^{, 29}$ in order to facilitate algorithmic intelligence (or "cognitive radio") to unfold its micro-time-critical efficiency, its dynamic intra-structure is not based on "time" anymore in the ontological sense but on an asynchronous grid of operative temporal actions, such as compressing movements into ultra-short slots. Such a mobile network replaces the immobile cables of the internet or telephone lines with a dynamic, in fact temporal grid for time-critical signal processing. Wireless communication is not simply a form of bridging spatial distances by electromagnetic waves between antenna and receiver anymore, but becomes primarily a function of intelligent shaping of signal events in between.

The traditional linear channel becomes itself dynamical and temporalized, composed of algorithms that "generate waveforms that support conjunctive pathways" - that is, a mobile infrastructure, a new kind of ether..$^{30}$ Although this almost amorphous infrastructure is still grounded in conventional communication engineering and technologies, in its essence it becomes more radically mathematized than even conceived by Shannon. ${ }^{31}$ While the traditional radio wave has been a time signal, in digital processing it becomes decomposed in frequency values in order to be treated computationally. The time axis itself is techno-mathematically suspended when a waveform that varies over time is transubstantiated into a set of 
component frequencies by Fourier transform; at the same time it allows for switching back from the frequency domain into the time domain. Such communication infrastructures oscillate between temporal and atemporal moments. Fourier transform is challenged by a structural incertitude either toward temporality or spatiality; in so-called wavelets, temporal linearity itself is suspended. For more efficient transmission streams, digital signals themselves become superimposed and enclosed in a signal envelope that looks like white noise, which is finally filtered back into a data stream. Temporality is just one (itself "ephemeral") function within such chains of operations. Efficient coding (convolution) has been developed to match signal errors and erroneous signal intrusions: "The stochastic character of the Viterbi algorithm ... alters the terrain on which machine time moves." ${ }^{22}$ What once was conceived as moments "in time" becomes a function of irruptions of the tempoReal (an escalation of Norbert Wiener's notorious term "time of non-reality" for the switching momentum between binary states ${ }^{33}$ ). Far beyond the world of communication engineering, knowledge culture has to acknowledge this nonsymbolic temporal quality, learning from media.

\section{Contemporary "Media Ecology" Identified from within Its Temporal Infrastructures}

In media-archaeological terms, computational technologies, in the very essence of both components (techné as well as lógos), consists of both "hardwired" temporality on the very infrastructural level of microchip circuitry and "soft-wired" temporalities resulting from what drives such machines: source code which concerns time-critical operations in computational languages such as assembly language. Micro-temporal physicality (the "real") is as much the object of media-archaeological analysis as "the symbolic" ordering of signal series, the algorithmic logic of digital culture-bypassing the imaginary alias "history."

The often debated "technological determinism" in media-archaeological analysis refers to infrastructures by necessity: a reconnaissance of both hardwired (materially embedded) and softwired (algorithmic) structures which govern media temporalities from within. Media archaeology is a consciously anachronistic identification of contemporary predefining states and layers of electronic media culture. The technological infrastructure of AM radio, for instance, endured for almost the whole twentieth century, notwithstanding the political and cultural catastrophes occurring within that century. The endurance of insisting hardwired and softcoded technologistics creates a 
temporal interval of its own. As long as such an epoché is still in operation, its media are excepted from the transience of the historical event. Such a theory of media time not only concerns emphatic "deep temporality" on a grand scale but also inversely re(oc)curs within the microscale of technological timing in the concrete circuitry of electronics itself.

Current "speculative design" theory registers a shift from progressive modernity to an epoché of contemporaneity where time is not an empty duration unaffected by the processes that happen within its technologies. On the extensive and microlevel of technological infrastructures, there is no homogeneous "time" but multiple and asynchronous tempor(e) alities, reminiscent of Ernst Bloch's notion of "non-contemporaneous contemporaneities" ("die Gleichzeitigkeit des Ungleichzeitigen”). The infrastructures of technological contemporaneity are not a coming together of data in time but a coming together of functional timings. Technological machine times challenge historicist notions of accumulative continuity. The concept of "media ecology" in that sense refers to temporal environments, to being in a clocked world, Heidegger's "Zeit des Weltbilds" taken literally.

\section{Notes}

1. "Zeitfunktionen der Signale": Karl Küpfmüller, Die Systemtheorie der elektrischen Nachrichtenübertragung (Stuttgart: Hirzel, 1974), 393.

2. See Gerhard Dohrn-van Rossum, History of the Hour (Chicago: University of Chicago Press, 1996).

3. Martin Heidegger, "The Age of the World Picture," in The Question Concerning Technology, trans. William Lovitt (New York: Harper \& Row, 1977), $115^{-154}$

4. Walter Ong, The Technologizing of the Word (London: Methuen \& Co., 1982).

5. See Barry Powell, Homer and the Origin of the Greek Alphabet (Cambridge: Cambridge UP, 1991).

6. Walter Benjamin, The Work of Art in the Age of Its Technological Reproducibility and Other Writings on Media, ed. Michael William Jennings, Brigid Doherty, and Thomas Y. Levin (Cambridge, MA: Harvard University Press, 2008).

7. Edmund Husserl, On the Phenomenology of the Consciousness of Internal Time (1983-1917), ed. and trans. J. B. Brough (Dordrecht: Kluwer Academic Publishers, 1991).

8. Lewis Fry Richardson, "A Forecast-Factory," in Collected Papers of Lewis Fry Richardson, vol. 1, Meteorology and Numerical Analysis, ed. P. G. Drazin (Cambridge and New York: Cambridge University Press, 1993), 219. 
9. See Manuela Garretón and Diego Gómez Venegas, “Towards an Archaeology of Media and Visual Languages," in The Counterculture Room: Pavillion of Chile at the London Design Biennale 2016, ed. FabLab Santiago (Barcelona: Ediciones Poligrafa, 2016), 108-127.

10. George Kubler, The Shape of Time: Remarks on the History of Things (New Haven, CT: Yale University Press, 1962).

11. Alan Turing, "Lecture to the Mathematical Society on 20 February 1947," in A. M. Turing's ACE Report of 1946 and Other Papers, ed. B. E. Carpenter and R. W. Doran (Cambridge, MA: MIT Press, 1986), 111.

12. However, Linus Torvald himself argued in favor of such time-critical syncopic interpolations. See Robert McMillan, "Linux's Creator Wants Us All to Chill Out about the Leap Second," Wired, January 9, 2015, http://www.wired. com/2015/01/torvalds_leapsecond/.

13. Geert Lovink, Dark Fiber: Tracking Critical Internet Culture (Cambridge, MA: MIT Press, 2002), 152. For more, see the chapter "Net.Times, Not Swatch Time: 21st-Century Global Time Wars," 142-159.

14. Lovink, Dark Fiber, 143.

15. Raymond Williams, Marxism and Literature (New York and Oxford: Oxford University Press, 1977), 122. See Shannon Mattern, "Deep Time of Media Infrastructure," in Signal Traffic: Critical Studies of Media Infrastructures (Urbana, Chicago, Springfield: University of Illinois Press, 2015), 71-93.

16. As stated in a lecture by Jussi Parikka, "Of Queues and Traffic: Network Microtemporealities," on occasion of the symposium Digital/Social Media and Memory, University of Glasgow, April 17, 2013.

17. Christoph Neubert, "Elektronische Adressenordnung," in Die Adresse des Mediums, ed. Stefan Andriopoulos, Gabriele Schabacher, and Eckhard Schumacher (Köln: DuMont, 2001), 34-63, 41.

18. Wikipedia, s.v. "Real-Time Web," accessed January 20, 2010, http://en.wikipedia.org/wiki/Real-time_web.

19. "Früher ging es um die Schaffung von Räumen ... heute geht es um die Zeit selbst, um Chronos, um die Kunst der longue durée." Geert Lovink, "Was uns wirklich krank macht," Frankfurter Allgemeine Zeitung, June 21, 2010, 27.

20. Othmar Kyas, Internet: Zugang, Utilities, Nutzung (Bergheim: Datacom, 1994), 65 .

21. Adrian Mackenzie, Wirelessness: Radical Empiricism in Network Cultures (Cambridge, MA: MIT Press, 2010), 65.

22. Letter to Barbara Ward, February 9, 1973, in Letters of Marshall McLuhan, ed. Matie Molinaro, Corinne McLuhan, and William Toye (Oxford: Oxford University Press, 1987), 466.

23. See Rainer Bayreuther, "Phänomenologische Grundlegung," in Heidegger-Handbuch: Leben, Werk, Wirkung, ed. Dieter Thomä, Katrin Meyer, and Hans Bernhard Schmid (Stuttgart and Weimar: Metzler, 2013), 509-512.

24. Eleni Ikoniadou, The Rhythmic Event: Art, Media, and the Sonic (Cambridge, MA: MIT Press, 2014). 
25. Shintaro Miyazaki, "Algorithmics: Understanding Micro-Temporality in Computational Cultures," Computational Culture 2 (2012).

26. Michel Foucault, The Archaeology of Knowledge, trans. A. M. Sheridan Smith (New York: Pantheon, 1972).

27. Mackenzie, Wirelessness, 72.

28. This becomes apparent from topics like "Algorithms and Modeling for Tracking and Locating Mobile Users" in the call for papers to the 1oth International Conference on Wireless and Mobile Network (WiMo 2018), May 26-27, 2018, Vienna.

29. Mackenzie, Wirelessness, 70.

3o. Mackenzie, Wirelessness, 66.

31. Claude E. Shannon and Warren Weaver, The Mathematical Theory of Communication (Urbana: University of Illinois Press, 1949).

32. Mackenzie, Wirelessness, 80.

33. See Claus Pias, "Time of Non-Reality: Miszellen zum Thema Zeit und Auflösung," in Zeitkritische Medien, ed. Axel Volmar (Berlin: Kadmos, 2009), 267-279.

\section{About the Author}

Wolfgang Ernst is Full Professor for Media Theories at the Institute for Musicology and Media Science at Humboldt University in Berlin. Having been academically trained as a historian and classicist (Latin Philology and Classical Archaeology) with an ongoing interest in cultural temporalities, Ernst grew into the emergent technology-oriented "German school" of media science. His academic focus has been on archival theory and museology, before attending to media materialities. His current research covers "radical" media archaeology as method, the epistemology of technológos, the theory of technical storage, the technologies of cultural transmission, micro-temporal media aesthetics and their chronopoetic potentials, and sound analytics ("sonicity") from a media-epistemological point of view. Ernst's latest booklet in English is entitled Technológos in Being. Radical Media Archaeology and the Computational Machine (2021). 



\section{Part II}

Microtimes 



\title{
5. Infrastructuring Leap Seconds: The Regime of Temporal Plurality in Digitally Networked Media
}

\author{
Isabell Otto
}

\begin{abstract}
The chapter pursues the hypothesis that the plurality of time in an age of digital interconnectivity imposes itself as a time regime to human and nonhuman entities. By looking at user practices, conventions of time measurement, and temporal operations of digital technologies it is argued that an infrastructural/infrastructuring process consists of the continuous weaving of a relational assemblage between different temporalities, which does not harmonize them, but makes them relevant to each other in their heterogeneity. Thus, the time regime of digitally networked media does not consist of the power constellation of an absolute, "true," measurable time, but of a fundamental plurality, which becomes visible on the basis of invisible processes and by that challenges all practices of temporal ordering.
\end{abstract}

Keywords: time regime, time measurement, temporal ordering, interfacing, practices, leap second

"Enjoy the moments of your life." With this slogan, the video app Leap Second promises to keep a special kind of diary: App users are invited to create one-second videos, select the best second for each day, and compile the individual seconds of the day into video diaries. This way they are able to review the days of a month or a whole year in seconds, and finally share these quotidian, yet outstanding moments of their lives via social media. On Instagram the app is advertised with small example videos: In seconds, outdoor and indoor shots alternate, slower and more eventful 
images, landscapes and portraits; shots of people toasting to each other at dinner are replaced by video snaps from an airplane; streets and houses abruptly change to shots of a workplace at home. The videos present a colorful and varied mixture of lived time. ${ }^{1}$

I would like to follow up this example with observations on three aspects that will interest me in the following chapter. First, the one-second videos refer to the relativity of human perception of time. The "second-days" seem to take different lengths. The moving image to which they are compiled appears homogeneous and clocked, but at the same time discontinuous, depending on the very different processes and situations in the individual videos, such as movement, actors, sound, color, light, and weather conditions. The homogeneous timing results from the technical settings of the app: The moments of the day are fitted into the almost imperceptibly fast succession of 100 centiseconds. In relation to the discontinuous moving images, the second appears as a reliable, inexorable, even absolute measure of a technically clocked time that forms and orders human perception of time.

But the app's name, Leap Second, probably quite unintentionally, indicates that such a dichotomous juxtaposition does not meet in any way the socio-technical and infrastructured temporalities under the conditions of digitally networked media. This is my second observation: the leap second is a phenomenon that not only emphasizes the meaning of a second, but also reveals the relativity of all systems of chronology and time measurement. As I will explain in more detail below, the pluralization of time measurement systems, which inevitably goes hand in hand with this relativity, becomes particularly precarious under the digital condition of temporality.

Thirdly, as Leap Second shows, all this has to do with the temporality of infrastructures and the infrastructures of temporality. The visual interface of the app bundles disparate and diverse processes of an ordering reference to temporality. The videos refer to natural time cycles, in which people are involved, when they picture the change from day to night or from season to season. The seconds (or centiseconds), on the other hand, refer to the socio-technical timing of clock time. Below these visible processes, however, the fabrication of different temporalities takes place on an infrastructural level, on the basis of micro-temporal and time-critical software processes. ${ }^{2}$ Beyond the displayed encounters of temporalities, an "inter-facing" between human users and nonhuman software and hardware components is taking place. ${ }^{3}$

An interfacing in this sense is less a spatial and temporal intersection, less a fixed thing in between than a process that fabricates the togetherness 
of disparate entities, or precisely: the being together "in time" (and "in permanence") of entities, each with its own proper time. This infrastructural and infrastructuring process consists of the continuous weaving of a relational assemblage between disparate entities and their different temporalities, which does not harmonize them, but makes them relevant to each other in their heterogeneity and plurality, in the first place. ${ }^{4}$ Apps such as Leap Second thus indicate a problem which they equally offer to solve.

With these preliminary considerations in mind, I would like to pursue the following questions: How are human, natural, and technical temporalities confronted with each other under the condition of digital technologies? And how are they becoming identifiable and problematic for each other in a processual temporality of infrastructuring? I pursue the hypothesis that the plurality of time in an age of digital interconnectivity imposes itself as a time regime to human and nonhuman entities. It is not only human and social temporalities that are plural. We can find a similar plurality in all orders of measured time. In a first step I would like to look at the plurality of digitally conditioned temporality from the perspective of user practices.

\section{Demands of Digitally Infrastructured Temporalities and Resistant Tactics}

In his critical reckoning with the early utopias of the net culture in Zero Comments, Geert Lovink sketches a differentiated picture of so-called "Internet time." On the one hand, he states, there are the practices of internet users who ideally behave "indifferent to time," when they spend time online or rather surrender themselves to the "luxury to get lost" and losing time as "data dandies" strolling through the net, contradicting all imperatives of effectiveness. On the other hand, Lovink observes the work processes of the IT industry, whose cooperation extends to different time zones. Global cooperation shows most clearly that there is "no simple synthesis of the local with the global." An "enhanced global time awareness" is necessary, "an awareness of other times. ${ }^{\prime 6}$ Lovink proposes to examine the requirement or even the demand to be confronted with a plurality of different times under the condition of digitally networked work as "time regimes under which today's Internet user are actually operating."7 This temporal plurality not only arises in the cooperation across time zones and in the spatiotemporal delimitation of work, which the internet makes 
possible and demands, but is also evident in the everyday practices of digital networking:

The online session is perhaps the best time unit to express what time on the Internet could look like. Think of more sessions happening simultaneously, such as chatting, talking on Skype, surfing MySpace, watching videos, following blog links, reading and answering incoming e-mails, and conducting a search. When you are online all the time (with a DSL broadband Internet connection), it is the bundle of these never-ending sessions that defines the Internet experience. ${ }^{8}$

Lovink believes, in 2008 , that digitally networked time can be limited to time units of being online. Digital time in this perspective is confined to a temporal refugium of the internet in which different proceedings run simultaneously but are separated from the "offline-world." However, he designs the scenario of "never-ending sessions," which is more appropriate for the current situation, because the technological condition of digital interconnectivity allows users to be online all the time.

It is exactly this state that current countermeasures of various "digital detox" movements want to deal with. "Participants gain insight into personal lifestyle techniques and practices that keep them grounded and connected even in the most stressed, overwhelming and technologically driven times," says the invitation of a digital detox organization, which has dedicated itself to the goal of (re)establishing some sort of balance in the digital age and offers device-free events, workshops, and retreats in nature, with the slogan "Disconnect to Reconnect." A recreational holiday at a California camp promises, according to its rules, a withdrawal on several levels: "No Digital Technology - No Networking — No Phones, Internet or Screens-No Work-Talk-No Clocks-No Boss-No Stress-No Anxiety-No Fomo (fear of missing out)." 9

It is striking how much the digital detox movement refers to temporal aspects in its diagnosis of the current situation: We suffer from the compulsion to have to respond immediately to messages, to constantly check the input of new messages; we have to keep pace with the speed of networked communication without finding time to draw breath. The plurality of constant, simultaneous, and far-too-fast processes is, according to these diagnoses, a characteristic of a new time regime of digitally networked media. There is no doubt that digital detox does not offer the prospect of a renunciation and definitive liberation from a digitally networked life. Disconnection is carried out for the purpose of better reconnecting afterwards. The temporary 
voluntary exclusion from a networked community follows the logic of permanent connectivity and affirms it, as Urs Stäheli has shown. ${ }^{10}$ "Digital detox" aims at the formation of subjects who can better adapt themselves to the demands of a "hardwired time" of digital networks and meet them in a self-regulatory way.

The same can be seen in the advice literature on self- and time-management. Typical here is the recommended way of dealing with the synchronous and asynchronous forms of communication that characterize a digitally networked workplace and that time management literature wants to optimize. This is where the plurality of diverse temporalities becomes apparent-especially in the description of badly handling emails and instructions for a correct way to do so. The time management literature suggests strategies for getting a grip on the "flood of e-mails" that characterizes every workplace. "Why am I not able to work because of all these e-mails?" A first answer to this question comes from a time-management guide using the Microsoft Office Outlook mail and calendar program, which argues for protecting the worker's proper time: “Don't be distracted all the time. Answer consciously and deliberately, instead of always reacting immediately to every message." ${ }^{\text {11 }}$

The educational program of time-management literature is not about a complete correction of a work situation characterized by too many temporally diverging and accelerated processes, disturbances, and interruptions, but about a better adaptation of the working subject to new technological (work) environments. The addressing of the subject is neoliberal and governmental: the aim that the subject voluntarily concerns itself with a safeguarding of proper relaxation and recovery times, which guarantee a better integration into a technological-economic power constellation. The supposed resistance strategies of digital detox and time-management are rather strategies of regulation, which refer to a techno-environmental condition without being able or wanting to change it. But how is the "ecology" of these practices to be characterized, ${ }^{12}$ the environment of human and nonhuman, natural and technological procedures that surround the practices and are regulated to govern subjects or enable self-government? What processes and constellations of power are inscribed in the regime of plurality? What are the conditions of possibility for the regime of plurality?

To determine this more precisely, a software developer is assumed whose practices of digitally networked collaboration across time zones could be directed by self-governmental regulation, such as digital detox or time management. In the course of a working day, when she not only writes the code for a new application, she communicates with designers, customers, 
fellow developers, and hardware companies scattered around the planet in different time zones. She not only has her own physical temporality and energy phases to consider, as Lovink cites an observer of working in the IT outsourcing industry, the "diurnal cycle of the human animal,"13 but also has to develop an increased awareness of the temporalities of her colleagues. The plurality of time is also conveyed by the parallel processes of synchronous and asynchronous processes of analogue and digital working communication through email, video chat, letter post, inhouse messaging, memo, meetings or telephone calls, in which different analogue and digitally networked devices (computers, smartphones, tablets, watches) are included. Finally, temporal plurality is conveyed to her in the practices of programming, which have to be oriented toward the different simultaneous processes of the computer.

The multiplicity of time is not necessarily tied to digitally networked media and could also be described from the sociological perspective of Barbara Adams, who sees a variety of other times included in the shaping of social time, "a multitude of times which interpenetrate and permeate our daily lives," including memories, anticipations, travel, and mobile working hours, as well as weather conditions and temporalities of the involved media. ${ }^{14}$ This multiplicity confronts the software developer just as it did an accountant around 190o. But I want to argue, following Lovink's observations, that under the condition of digitally networked media the plurality of time becomes a time regime on which regulating strategies of the adaptation of subjects orientate themselves, because of a specific relationship of visibility and invisibility that characterizes the temporal ordering of human subjects confronted with the temporalities of digital infrastructures. This means that digital time cannot be realized at all as a liberation from the dictate of time measurement, as imagined in visions of "network time." In Instead, social temporalities are structured and challenged by the (micro-)temporalities of digital infrastructures. Additionally, there can just as little be a new standard time established that tames this plurality of time. The plurality of time cannot be suspended.

We are dealing with a specific form of (in)visibility of plural spacetime systems: The time regime of digitally networked media consists in a visibility of different spatiotemporal orders that run simultaneously but independently of each other and that are perceptible for human users in processes of interfacing. This visibility, which is by no means limited to visuality and thus to graphical user interfaces, is conditioned and made possible by the constitutive invisibility of digital processes that take place in discontinuous pulsing below the threshold of human perception 
and thus evoke the impression of "real time" without identifying the synchronization and coordination necessary for producing visibility and continuity. ${ }^{16}$ In digital processes, the transmission time of a time signal that connects two independently running space-time systems is (for human observers) imperceptibly short. This is precisely the basis of the universal time fictions from the early internet era. For example, Swatch has for some time pursued the goal of establishing a globally uniform time order measured in beats. But the time regime of the digital does not consist of a standard time.

Digital interconnectivity brings independently running time orders into a relationship of mutual visibility and disturbability. In this way, the relativity and contingency of any time system become recognizable. The time regime of digitally networked media does not consist of the power constellation of an absolute, "true," measurable time, ${ }^{17}$ but of a fundamental plurality, which becomes visible on the basis of invisible processes and by that challenges all practices of temporal ordering and synchronization. In this sense, the time of digital media is not characterized by a multiplicity of time, which enables new creative developments, but by a time regime that requires an increased sensitivity for the relativity and plurality of time.

Our imagined software developer, based in Vienna, who works under the condition of digital networked working environments, knows that for her colleague in Australia, with whom she is having a Skype call, different spacetime conditions prevail than for herself. Daytime and season are completely different. But the imperceptible processes enabled and conditioned by the infrastructures of digital networks are what make this other time visible and audible on her device in a process of interfacing, a space-time system that appears simultaneous to her own spatio-temporality, but nevertheless is perceptibly different. This demands a temporal plurality from her and challenges her to adapt her practices - perhaps when her meeting is in the morning of Sydney local time and she has to fight tiredness because for her it is 11 p.m. Simultaneous temporal orders are no longer independent of each other under the condition of digital interconnectivity, but become visible and relevant to each other. The relativity and contingency of temporal orders become apparent, the locally and diurnally different observations and experiences of time. As will still be seen, this applies not only to human perceptions of time and practices of temporal order, but also to the technical processes of digital connectivity. However, it is fundamental that relativity and contingency are inscribed in every measurement of time. I will further explore this point using the example of the measurement of the second in the next step of my argumentation. 


\section{The Measurement of the Second and the Fleeting Stability of Time Orders}

Social time orders in the form of clocks, work plans, and calendars form time regimes that demand self-regulating adaptation of subjects to economic structures. ${ }^{18}$ However, the clock is not a once and for all stable technical timepiece that regulates the social realm, a determining, inanimate time technique that threatens and destroys living, subjective times. Rather, each time measurement is based on a "technicity" in the sense of Gilbert Simondon as the "degree of the object's concretization,"19 which stabilizes a spatiotemporal coordination of socio-technical collectives, but also constantly keeps them open for restructuring and, despite an increasing precision of technical time measurement and standardization, can only establish a temporarily stable structure. ${ }^{20}$ The fact that the continuous restructuring of time regimes is taking place on the basis of a changing technological condition becomes particularly clear in the current debate on the leap second.

Since 1972, an additional second has been inserted at irregular intervals into the Coordinated Universal Time (UTC) to compensate for fluctuations and a gradual slowdown in the earth's rotation. Due to its relation to the sun's position, UTC is still regarded as "natural" and "appropriate" for living beings on earth. Measured on the basis of atomic clocks, UTC is slowed down by the leap second in such a way that it never deviates by more than 0.9 seconds from a time measurement oriented at the position of the sun, the rotation of the earth, or the orbit of the earth. This deviation is determined by the International Earth Rotation and Reference System Service by constant observation or measurement. The service then decides whether the day is one second longer at the end of June or December, i.e., a 61st second - $23 \mathrm{~h}$ $59 \mathrm{~m} \mathrm{6os}$ - is inserted into the UTC at the end of the day. Without the irregular insertion of leap seconds, according to a fear of the unpredictable development of the difference between the Earth's rotation and atomic clocks, the deviation could be four hours in 2000 years. Even further in the future, the clocks might indicate noon when it is in the middle of the night. ${ }^{21}$

It is important to recognize that the leap second problem has arisen only from timekeeping practices that are part of a continuous restructuring of clock time and its basal unit of measurement, the second. The leap second is a metastable remnant that results in a "supersaturation" of the current standard time system and requires its restructuring. ${ }^{22}$ The leap second thus arises in the course of a technicity of measured time, which inscribes into each time regime an openness to restructuring: With the beginning 
of time measurement by atomic clocks, the "power/knowledge" regime of clock time, the political and institutional competence shifts as well as the expertise between astronomy and atom physics. ${ }^{23} \mathrm{~A}$ decisive aspect of this shift is the refinement and stabilization of time measurement by atomic clocks. From the mid-1950s onwards, the physical determination of time by measuring the transitions between levels of the atom's ground state was a new way of separating the temporal order from planetary constellations. Until 1956, the second was determined by the Earth's rotation around its own axis, i.e., as a fraction of the mean solar day, and was then replaced by the ephemeris second, which is oriented at the Earth's orbit around the sun and which was considered to be more stable, then. Quite in contrast to the irregularities of astronomical time measurement, the period duration of an electromagnetic radiation absorption or emission in the transitions in the ground states of an atom, proves to be-at least in principle-constant. Physicists in the middle of the twentieth century, using the cesium atom, determined the length of a second that remains valid until today, defined by the Bureau International des Poids et Mesures (BIPM): "The effect of this definition is that the second is equal to the duration of 9192631770 periods of the radiation corresponding to the transition between the two hyperfine levels of the unperturbed ground state of the ${ }^{133} \mathrm{Cs}$ atom." ${ }^{24}$

The new precision of time measurement entails some consequential problems because the accuracy of the measurement makes it obvious that every time measurement is relative and situational. In the atomic age of time measurement the irregularities of universal world time become visible: not only the relativity of time, but also the irregularities of each time measurement that is oriented at the position of the sun, the rotation of the earth, or the orbit of the earth. Basically, inaccuracy also applies to atomic time measurement. However, the determination of the second, which is nevertheless accurate to microseconds, can show that the mean solar day of the astronomically calculated UT1 (the current equivalent of Greenwich Mean Time) is 2 milliseconds longer than the day calculated from atomic seconds: it comprises $86,400.002$ instead of exactly 86,400 seconds. The atomic clock thus becomes a (temporarily) more stable and precise clock only in comparison with other clocks, which can represent the course of time less constantly. This difference between the atomic and astronomical time scales, visible in the precision of atomic time, gives rise to the UTC regime and, with it, the need to introduce leap seconds that keep this difference within a tolerance range in practices of continuous balancing. In favor of a uniform time order, the leap second prevents two time scales from drifting apart, but at the same time it inscribes a discontinuity into this uniform time order, which 
in the case of digital networking is clearly recognizable as a problematic uncertainty. As a sign of its technicity, the leap second keeps universal time open and changeable. Its potential to interconnect the macro-cosmological environment of the astronomical and the micro-cosmological environment of atomic time measurement can thus only temporarily lead to a stable order of time. A stability that, under the condition of digitally networked media, is currently about to turn into instability. While the leap second has guaranteed a coordination with, or adaptation of, socio-technical to planetary-organic processes for more than 40 years, thus enabling a collective of human and nonhuman entities to be together "in time," it now fails in the confrontation with digital media and processes that are not only time-dependent, but time-critical. ${ }^{25} \mathrm{~A}$ restructuring of the time regime of a universal world time is necessary, which in the interaction of political, economic, and scientific interests will probably find a new, but just as temporary answer to this problem. In the third step of this chapter I will argue, that the plurality of time not only challenges social practices of time ordering but also digital devices and systems.

\section{Leaping Seconds and Digital Interconnectivity}

The plurality of the now coexisting different orders of time measurement is particularly visible in the problems of networked computer systems. One of the oldest internet protocols was developed by David L. Mills, who calls himself an "Internet timekeeper." ${ }^{\text {"6 }}$ To this day, the so-called Network Time Protocol is used and ensures clock synchronization on the internet. It is based on the coordinated world time and inserts leap seconds. The Network Time Protocol is part of the decentralized power structures of the internet as described by Alexander Galloway. ${ }^{27}$ It can be described as a kind of time management guide for digital devices because it allows computers to cope with the requirement of time plurality.

The protocol provides for a tree-like structuring from servers to clients, based on computer servers whose clocks are synchronized via precise atomic clocks. These in turn can be used as a reference for the coordinated world time. When a leap second is introduced, the time elapsing according to the Network Time Protocol is frozen for one second. Immediately after the introduction of the leap second, the system clock continues to run as if nothing had happened. It "forgets" the introduction of the leap second as well as the introduction of all previous leap seconds. Each new leap second generates a new time scale and thus pluralizes internet time successively. 
There are as many Network Time Protocol time scales as leap seconds historically introduced since 1972; therefore, each time the system's past is accessed, the time scale must change and the corresponding leap seconds must be subtracted again. ${ }^{28}$ The discontinuity of the coordinated world time thus multiplies in the historical course of the internet time based on the Network Time Protocol, which adapts to UTC again and again. ${ }^{29}$ The question arises as to what happens to computer processes within the "paused time" of the leap second, whether the processuality of computational time, which is based on discontinuities and caesuras, ${ }^{30}$ but is nevertheless constantly ongoing, can be stopped at all for one second. This is hard to imagine for complex, digitally networked systems, according to the argumentation of developers of the company Google:

Very large-scale distributed systems, like ours, demand that time be well-synchronized and expect that time always moves forwards. Computers traditionally accommodate leap seconds by setting their clock backwards by one second at the very end of the day. But this "repeated" second can be a problem. For example, what happens to write operations that happen during that second? Does email that comes in during that second get stored correctly? What about all the unforeseen problems that may come up with the massive number of systems and servers that we run? Our systems are engineered for data integrity, and some will refuse to work if their time is sufficiently "wrong." We saw some of our clustered systems stop accepting work on a small scale during the leap second in 2005 , and while it didn't affect the site or any of our data, we wanted to fix such issues once and for all. ${ }^{11}$

The solution for Google is to "smear" the leap second: an adjustment by milliseconds over a day. But would it not be desirable to abolish the leap second and move on to a continuous time measurement that completely detaches itself from the sun as the central timer? This question has been increasingly discussed since the beginning of the 2000s. As a trigger, an increased time sensitivity due to the (feared) "Millennium Bug" of numerous computer systems is very likely. The camp of supporters is growing steadily. In view of the changing technological conditions, a collective of authors that brings together the physical, astronomical, and geopolitical expertise of different institutions concludes that we should not hesitate to establish a binding time system that adapts to the modern technologies and needs of accurate time measurement in space travel, satellite navigation, metrology, telecommunications, and synchronization of networked computers. ${ }^{32}$ 
The fact that the International Telecommunication Union took up the debate and examined the feasibility of a continuous time scale at the World Radiocommunication Conference in 2015 is a clear signal for the shift in the time regime resulting from the current technological condition. Judah Levine, of the US National Institute of Standards and Technology (NIST), clearly voted as early as 2013 to refrain from inserting leap seconds in the future. Keeping the difference between coordinated world time and astronomically measured time as small as possible is too high a price to pay, given the massive difficulties that leap seconds entail for digitally networked systems:

The problems of time-ordering, causality and the ambiguity of time intervals in the vicinity of a leap second are not easily remedied because they arise in a fundamental way from the interaction of the binary representation used for time stamps and the occurrence of a positive leap second. During a leap-second correction, the time servers operated by NIST will receive approximately 150000 time requests when the time transmitted by the server is 23:59:59, and the increasing number of financial transactions that depend on millisecond-level timing are sure to be affected. ${ }^{33}$

However, no decision was made at the 2015 World Radiocommunication Conference. The evaluation of further studies and the consideration of a new time order were postponed until 2023. The abolition of the leap second could be identified as a subjection to a "regime of technology," as a "harder hardwiring" of temporality, that now dominates all natural and social processes; as an overhand gain of techno-economic processes that sets the pace and the need for precision for a binding world time. A world time without leap seconds-would that be an order of time that makes a (planetary, organic) outside of technological processes irrelevant? A closer look at the ecology of the leap second, its integration into a network of atomic, planetary, organic, social, and technological relations, has shown, however, that this description would be too short-sighted. In a final step I would now like to specify my thesis of temporal plurality.

\section{The Time Regime of Plurality}

What the perception of different temporalities in (work-)processes of global interconnectivity makes just as clear as the drifting apart of astronomical 
and atomic orders of time, is the fundamental relativity of each regularity of time. Influenced by the theory of relativity, which he received as a "new theory" in 1920, Alfred North Whitehead already clearly summed up this fact at the beginning of the twentieth century in his natural philosophic work Concepts of Nature and warned against a confusion:

According to the new theory, there are an indefinite number of discordant time-series and an indefinite number of distinct spaces. Any correlated pair, a time-system and a space-system, will do in which to fit our description of the Universe. We find that under given conditions our measurements are necessarily made in some one pair which together form our natural measure-system. The difficulty as to discordant time-systems is partly solved by distinguishing between what I call the creative advance of nature, which is not properly serial at all, and any one time-series. We habitually muddle together this creative advance, which we experience and know as the perpetual transition of nature into novelty, with the single-time series which we naturally employ for measurement. ${ }^{34}$

If one avoids this misunderstanding and distinguishes between process-as the term Whitehead uses in his major work Process and Reality, instead of "creative advance of nature"35 — and (measured) time, one can thus doubt that two observers mean the same thing when they determine space and time from their own perspectives. Each measurement of time must therefore produce a different order of time. If one assumes, with Whitehead, that space and time (in the measurable sense) are only possibilities to express certain truths about the relations between constantly becoming entities within the basic process of all existing things, but that there are numerous truths corresponding to the numerous space-time systems, time orders such as clock time or coordinated world time must be particularly powerful and momentous orders that are temporarily capable of forming regular time regimes, time regimes that combine chronopolitical with geopolitical interests and form and sustain cultural or social sequences and practices. ${ }^{36}$

However, the technicity of time measurement, which becomes recognizable by the leap second, introduces time as a fundamental process of becoming and passing into every order of time and prevents its complete fixation. It thus focuses on the condition of power relations and normalization processes of a unified temporal order. Each practice of time ordering is to be viewed in the context of its "ecology of practices"377 and develops its own truth there, which is always only one within a plurality of other "true" time orders taking place in parallel, which this practice must blank out and from which it must 
detach itself in order to justify itself as "true." A critique of power in the sense of a "cosmopolitan politics" has to return the practices of time measurement to their situational interdependencies of human and nonhuman processes and their mutual dependencies, relations and affiliations. ${ }^{388}$ Those who want to secure access to time "in itself" through operations of measurement must make productive what Whitehead characterizes as a misunderstanding: the identification of time as a temporal regularity that is temporarily stabilized, and time as a fundamental processuality that permeates every stabilization. The implementation of a standard time with a universal claim can thus be described as a gesture of power, as a power/knowledge regime in the sense of Michel Foucault, which helps a temporal order to gain hegemony and which - as in the case of clock time and its standardization in a universal world time since the end of the nineteenth century-represents the condition for a normalization and naturalization of this one possibility of temporal order, detached from its situational contexts of measurement. This procedure is, however, supported by the socio-technical production of a measuring and abstracting-calculating access to time itself, which changes on the basis of the changing technological condition, but which must suppress this change in favor of a universalization of time. "Physicists feel weak and they protect themselves with the weapons of power, equating their practice with claims of rational universality." 39

Digital infrastructures provide a constellation in which the relativity of temporal regularity becomes visible and the assertion of an identity between time order and "natural" time "in itself" is no longer a necessary argument for establishing a binding standard time. The coupling of power and truth is replaced by a combination of power and neoliberal economic expediency that knows about its contingency. The abolition of the leap second and the introduction of a universal time running constantly over atomic seconds would not be a final solution, not an order of time that would be adequate for a digital temporality once and for all, but only a temporary stabilization, another time regime that has emerged from an ecology of atomic physics, astronomy, and IT practices and that differs from previous regimes (e.g., Greenwich Mean Time) by a clear reference to the relativity of time and to the plurality of possible time measurements. However, the debate about the leap second shows a circumstance that is of highest relevance for the investigation of a temporality of infrastructures: The temporal processes in systems of digital data transmission do not take place in a refugium that completely excludes them from their cosmological environment - the radiation emissions of the cesium atom or the gravitational fields and rotations of the earth. Rather, digital processes are part of this environment, 
they are infiltrated into it, shape and alter it, and determine the orders of time that can temporarily stabilize on its basis. ${ }^{400}$ In a time of digital interconnectivity, temporality is conditioned by a technologically shaped environment, by a media ecology from which users, technical objects, and data networks emerge with their respective orders of time, by a web of relations that runs through and crosses every socio-technical order of time.

The discrete processuality of digital infrastructures as a condition of possibility for the simulation of perceptible digital objects justifies speaking of a specificity of a digital time that characterizes our present condition through digitally networked media that permeate all areas of life. This characteristic is insufficiently captured with a reference to the multiplicity of digital time. For the description of an experiencability of manifold inherent times - of the user, the device, the software, the network — all of which are related to one another and perceived as a multi-temporal web, leaves open why this should be new or special under the condition of the digital. The differentiation of a time of digital interconnectivity within the fundamental plurality of time, according to my thesis, lies in a specific visibility of or disturbability by the plurality of time at the level of technical operations und user practices. The digital process causes the perceptible appearance of a symbolized time, i.e., the perceptibility of images, sounds, or text elements on displays, in a way that is imperceptible to human beings, and thus undermines the difference in their spatial-temporal orders by the speed of digital processing undermining the perceptible low-frequency range. However, this happens without cancelling the difference of spatiotemporal orders. It is rather reduced to an imperceptibly small "in-between." Thus, time orders are confronted with each other that would otherwise run independently of and undisturbed by each other.

The time of digital networks thus does not multiply the temporalities themselves but the constellations in which different times are confronted with each other. A webcam image during a Skype call shows a different spatiotemporal situation, but it shows (simulates) it here and now as simultaneous and relevant to the practices of the user viewing it. This becoming visible for each other-or better: the becoming relevant of different time orders-takes place on the level of not only human perceptibility but also the infrastructure: the manifold hardware and software processes and system times. The relevance of temporal plurality is particularly apparent in the change of uniform time orders, such as the coordinated world time, or in the coordination of human and technical proper times. The confrontation with the plurality of different temporalities does not lead to a (harmonious) temporal fabric. Rather, the plurality of time under the condition of digitally 
networked media requires a constant sensitivity to the relativity of time and results in an increased necessity for coordination or (re-)ordering. This can be seen in the debate about the leap second as well as in the possibilities of the records of lifetime as promised by the app Leap Second. The diversity of time, visible in its manifold confluence, becomes a regime of plurality that constantly challenges the practices of ordering time anew.

\section{Notes}

1. See Jon Andersen @leapsecond, Instagram, https://www.instagram.com/ leapsecond/?hl=de, October 22, 2019.

2. Wolfgang Ernst, Chronopoetics: The Temporal Being and Operativity of Technological Media, trans. Anthony Enns (London and New York: Rowman \& Littlefield, 2016).

3. Alexander Galloway, The Interface Effect (Cambridge and Malden, MA: Polity Press, 2012).

4. For more on infrastructuring, see Susan Leigh Star and Geoffrey C. Bowker, "How to Infrastructure," in Handbook of New Media: Social Shaping and Consequences of ICTs, ed. Leah A. Lievrouw and Sonia Livingstone (London: SAGE Publications, 2002), 151-162.

5. Geert Lovink, Zero Comments. Blogging and Critical Internet Culture (New York and London: Routledge, 2008), 120.

6. Lovink, 121, 126.

7. Lovink, 118.

8. Lovink, $122 \mathrm{f}$.

9. Digital Detox Retreats, http://digitaldetox.org/retreat, October 22, 2019.

10. Urs. Stäheli, "Entnetzt euch! Praktiken und Ästhetiken der Anschlusslosigkeit," Mittelweg 36 22, no. 4 (2013): 3-28.

11. "Lassen Sie sich nicht dauernd ablenken. Antworten Sie bewusst und überlegt, anstatt auf jede Nachricht immer sofort zu reagieren." Lothar Seiwert, Holger Wöltje, and Christian Obermayr. Zeitmanagement mit Microsoft Office Outlook. Die Zeit im Griff mit der meistgenutzten Bürosoftware-Strategien, Tipps und Techniken (Versionen 2003-2010), 8th ed. (Unterschleißheim: Microsoft Press, 2010), 13.

12. See Isabelle Stengers, "Introductory Notes on an Ecology of Practices," Cultural Studies Review 11, no. 1 (2005): 183-196.

13. Lovink, 120.

14. Barbara Adams, Timewatch: The Social Analysis of Time (Cambridge: Polity Press, 1995), 12.

15. Robert Hassan, "Network Time," 24/7: Time and Temporality in the Network Society, ed. Robert Hassan and Ronald E. Purser (Stanford, CA: Stanford University Press, 2007), 37-61. 
16. See Friedrich Kittler, "Real Time Analysis-Time Axis Manipulation," Zeit-Zeichen: Aufschübe und Interferenzen zwischen Endzeit und Echtzeit, ed. Georg Christoph Tholen and Michael O. Scholl (Weinheim: Acta humaniora, 1990), 363-377; and Esther Weltevrede, Anne Helmond, and Carolin Gerlitz, "The Politics of Real-Time: A Device Perspective on Social Media Platforms and Search Engines," Theory, Culture \& Society 31, no. 6 (2014): $125^{-150 .}$

17. Clark Blaise, Time Lord: Sir Sanford Fleming and the Creation of Standard Time (New York: Vintage, 2002).

18. Colin Symes, "Chronicles of Labour. A Discourse Analysis of Diaries." Time \& Society 8, no. 2 (1999): 357-380.

19. Gilbert Simondon, On the Mode of Existence of Technical Objects (Minneapolis, MN: Univocal, 2017 [1958]), 72.

20. Adrian Mackenzie, "The Technicity of Time: From 1.00 oscillations/sec to 9,192,631,770 Hz," Time \& Society 10, no. 2-3 (2001): 235-257.

21. Robin McKie, "Time Running Out for 'Leap Second' That Has Kept Us in Step with Our Slowing Planet," Guardian, January, 15, 2012, http://www. theguardian.com/science/2012/jan/15/time-leap-second-atomic-clock.

22. See Gilbert Simondon, "The Position of the Problem of Ontogenesis," Parrhesia 7 (2009 [1989]): 6.

23. See Michel Foucault, Power/Knowledge. Selected Interviews and Other Writings, ed. Colin Gordin (New York: Pantheon Books, 1980).

24. Bureau International des Poids et Mesures (BIPM), Measurment Units, https://www.bipm.org/en/measurement-units, October 26, 2019.

25. See Ernst, Chronopoetics.

26. David L. Mills, "A Brief History of NTP Time: Confessions of an Internet Timekeeper," ACM Computer Communications Review 33, no. 2 (2003), http:// www.eecis.udel.edu/ mills/database/papers/history.pdf.

27. Alexander R. Galloway, Protocol: How Control Exists after Decentralization (Cambridge, MA, and London: MIT Press, 2004).

28. See Mills, "Brief History of NTP Time."

29. As can be seen in RFC 8633 from July 2019, the Network Time Protocol is still a standard recommendation. It might be that the software clock synchronization system HUYGENS, which allows nanosecond accuracy, will change the fleeting stability of NTP in dealing the discontinuities of leap seconds. Yiling Gent, et al., "Exploiting a Natural Network Effect for Scalable, Fine-grained Clock Synchronization," Proceedings of the 15th USENIX Symposium on Networked Systems Design and Implementation, April 9-11, 2018, Renton, WA, USA, https://www.usenix.org/system/files/conference/ nsdi18/nsdi18-geng.pdf. I thank Armin Beverungen for this hint.

30. Georg Christopher Tholen, Die Zäsur der Medien: Kulturphilosophische Konturen (Frankfurt am Main: Suhrkamp, 2002), 141, 132.

31. Christopher Pascoe, "Time, Technology and Leaping Seconds," Google Official Blog, September 15, 2011, http://googleblog.blogspot.de/2011/og/ time-technology-and-leaping-seconds.html. 
32. Robert A. Nelson, et al., "The Leap Second: Its History and Possible Future," Metrologia 38 (2001): 509-529.

33. Judah Levine, "Impact of Leap Seconds on Digital Time Services: Internet Time Servers," ITU News 7 (2013), https://itunews.itu.int/En/4276-Impactof-leap-seconds-on-digital-time-services-BRInternet-time-servers.note. aspx.

34. Alfred North Whitehead, The Concept of Nature (New York: Cosimo, 2007 [1920]), 178.

35. Whitehead, Process and Reality: An Essay in Cosmology, Corrected Edition (1927-28), ed. David Ray Griffin and Donald W. Sherburne (New York: Free Press, 1978).

36. See Ian Klinke, "Chronopolitics: A Conceptual Matrix," Progress in Human Geography 37, no. 5 (2013): 673-69o.

37. Stengers, "Introductory Notes on an Ecology of Practices."

38. Stengers, $188 \mathrm{f}$. See also Cosmopolitics 1. I. The Science Wars. II. The Invention of Mechanics. III. Thermodynamics. (Minneapolis and London: University of Minnesota Press, 2010); and Cosmopolitics 2. IV. Quantum Mechanics. V. In the Name of the Arrow of Time. VII. The Curse of Tolerance (Minneapolis and London: University of Minnesota Press, 2011).

39. Stengers, "Introductory Notes on an Ecology of Practices," 196.

40. See Erich Hörl, General Ecology: The New Ecological Paradigm (London: Bloomsbury, 2017).

\section{About the Author}

Isabell Otto is Professor of Media Studies at the Department of Literature, Art, and Media Studies, University of Konstanz. Currently she researches on temporalization, media theory and digitally networked mobile communities. Her book Prozess und Zeitordnung. Temporalität unter der Bedingung digital vernetzter Medien is published by Konstanz University Press (2020). 


\title{
6. Life at the Femtosecond
}

\author{
Geoffrey C. Bowker
}

\begin{abstract}
This chapter argues that we are creating a fundamental new ontological layer which has far reaching social and political consequences. In this new "present," we need to account for ever higher time frequencies-since this is where decisions are being made which affect us all. In particular it argues that whereas the prior forms of capitalism concentrated on colonizing space, newer forms involve colonizing time.
\end{abstract}

Keywords: computer time, ontology, science studies, temporality, acceleration

Par la vitesse, le présent se transforme en éternité.

-François Hartog ${ }^{1}$

Time, loosely put, is the direction in which physics tells its best stories.

-Craig Callender ${ }^{2}$

And so, Ladies and Gentleman, as the Southwestern train of time is delayed by the points failure of predictability and the pissed off Poole-based passenger of perpetuity becomes trapped in the out-of-order train toilet of eternity, I notice it's the end of the show. -Jack Dee ${ }^{3}$

It is a well-trodden historical path to take Charles Babbage, through his design for an Analytical Engine, as the progenitor of the modern digital computer. This hindsight can make it harder to see an origin of computing in the production of machinery - the Engine was to be a vast machine, deploying techniques learned in his tour of machinery and manufactures

Volmar, A. and K. Stine (eds.), Media Infrastructures and the Politics of Digital Time: Essays on Hardwired Temporalities. Amsterdam: Amsterdam University Press, 2021 DOI 10.5117/9789463727426_CHo6 
in England in the 1820 s (weaving and watch production) in the heat of the industrial revolution and expressed not in binary code but in a symbolic language describing the functioning of machines. ${ }^{4} \mathrm{He}$ conceived it not only in terms of the time compression we associate with computing, but rather its opposite: "It is impossible to construct machinery occupying unlimited space but it is possible to construct finite machinery, and to use it through unlimited time. It is this substitution of the infinity of time for the infinity of space which I have made use of, to limit the size of the engine and yet to retain its unlimited power." 5 This infinite logical time was also-as this new language and as his analysis of time taken for operations in watch production in the 1820 - complemented by time compression: the ineluctable drive to make things happen faster and faster: "whenever the Analytical Engine should exist, all the developments of formula would be directed by this condition - that the machine should be able to compute their numerical value in the shortest possible time." ${ }^{6}$

Babbage's new technology, then, ushered in its own infinite time at the very moment when geology in the 1830 s invented a new infinity of time ${ }^{7}$-a consonance of which Babbage was deeply aware: variations in time scale from the fleeting life of the mayfly to the eons of geology were central to his imaginary. ${ }^{8}$ One of the tricks of the trade for digital computers is to collapse potentially infinite serial time through synchronization: running many operations at the same time and coordinating the results. ${ }^{9}$ Commands to go parallel, spawn processes, and sync results sit on top of the underlying serial structure. ${ }^{10}$ An extreme formulation of the substitution of space for time here is David Deutsch's description of quantum computing as a way of allowing tasks to be performed collaboratively across multiple universes ${ }^{11}$ - he reckoned that to factor a 250 digit number we would need to deploy $10^{500}$ different universes (the operation would run parallel in each, and the result would be given by interference) in almost no time-where Donald Knuth had estimated that it would take over a million years using a million computers. Time was for Deutsch a budgeting issue:

Evolution would never have got off the ground if the task of rendering certain properties of the earliest, simplest habitats had not been tractable (that is, computable in a reasonable time) using readily available molecules as computers. ... What computations, in other words, are practical under a given time and under a given budget? ${ }^{12}$

The parallelism of computing today - whether based in the cloud or under the hood of a PC in the form of multicore processors - trades between two 
temporalities, the speed of messaging (spatial) and the remorseless ticking of the computer clock (temporal; not highly scalable above current limits).

The fastest computers now can do about 16 petaflops (floating point operations) per second - that is 16 by $10^{15}$ operations. That is a whole lotta flops, even for an industry that romances the decimal point. ${ }^{13}$ It means that in fewer than one hundred seconds, it could do the equivalent of one calculation per second since the putative Big Bang. Roughly. On the other hand, in a deeply meaningless calculation, it has been asserted that the human brain performs between 10 and 30 times as many. ${ }^{14}$ The reason why this comparison is even a question is that the holy grail of artificial intelligence needed to replicate the work of many complex professions, such as scientist and psychiatrist-known as AI-complete programs-require complete emulation of the human brain in the interests of efficiency. ${ }^{15}$ If we succeed: "then it will become feasible for machines to carry out such jobs, and to do so more cheaply and more effectively than humans." ${ }^{\text {"6 }}$ Indeed: "if and when human-level AI is achieved, superintelligence will soon follow.... Even if human-level AI is achieved by the most conservative means-by slavishly copying nature - the resulting liberation from the speed restrictions inherent in biology is enough." The romance does not stop there-Murray Shanahan fantasizes: "A theoretically perfect computer with a mass of $1 \mathrm{~kg}$ and occupying a volume of 1 liter would perform $5.4 \times 10^{50}$ logical operations per second on $10^{31}$ bits." This is 39 orders of magnitude greater than today's computers. In the future, we might see a portion of space, as Shanahan quotes Hans Moravec, "rapidly transformed into a cyberspace, [wherein beings] establish, extend, and defend identities as patterns of information flow ... becoming finally a bubble of Mind expanding at near lightspeed."'17

We do not generally think of these kinds of speeds-our lives seem to flow at the rate of less than one thought per second and connectivity for many is so fast that it's basically just instantaneous, and so invisible. (The days of watching an email message unfurl painstakingly over a modem attached to a telephone line are long over.) And yet they affect us in our daily lives. They can irrupt - in the form, say, of the flash crash of 2010, where trading algorithms were making and changing bets at an astonishing rate, leading to a trillion dollar dive on Wall Street that lasted all of thirty-six minutes. More insidiously, very fast computing times are needed for the forms of machine learning being used by casinos, states, and advertising companies such as Facebook and Google to create the nudge technologies that cosset you along the path of life mostly without your being aware. ${ }^{18}$ Thus, Armin Beverungen and Ann-Christine Lange cite Katherine Hayles on the missing half second between perception (registering an event) 
and consciousness (processing it): “This cost [the delay] ... assumes new importance when cognitive nonconscious technical devices can operate at temporal regimes inaccessible to humans and exploit the missing half-second to their advantage."'19

Timothy Mitchell asserts that we are pillaging the past of its biological productions; he estimates that a single liter of petrol needs about 25 metric tons of ancient marine life, and that we burn 400 years of entire plant and animal life production a year. ${ }^{20}$ In our brave new age, we are also burning time at an ever faster rate - and this is just as ontologically, politically, and socially significant. Ian Osborne and Daniel Clery observed in 2004 that the "most important commodity" today is time. ${ }^{21}$ And the control of events at the very small scale is becoming central —as Paul Hegarty argues in his discussion with Gary Genosko of Google's attempt to "own" the smear of the leap second needed to cope with the irregularities of the earth (that perfect clock for the Enlightenment scientists): "Google's execution of time-critical processes establishes its mastery over the measurement and manipulation of humanly imperceptible micro-temporal events. ${ }^{\prime 22}$ And it all adds up - by one estimate, the world will have spent a billion years online in $2018 .^{23} \mathrm{As}$ Wolfgang Ernst says, we need to take machine time seriously. ${ }^{24}$

The argument here will be twofold: first that we are creating new ontological layers of the socionatural through the development of computing technology and second that the associated temporalities are richly textured, that is, not all about speed.

\section{Speed, Acceleration and Delay}

High-frequency trading is always a good spot to find computers and networks working at their fastest. A network switch made by Metamako "allows a trade order to be placed in the time it takes a photon to travel 90 feet" ${ }^{25}$ - and there is a market, these switches in 2016 were selling at 100 units a month. We need shorter times. This can reach fantasy proportions: "When in 2012 scientists briefly thought they'd detected neutrinos that could travel faster than light, for example, high-frequency traders pondered how they might build a system that would execute trades that would, theoretically, occur in the past." ${ }^{26}$ In this current imaginary, it really is only a step from high energy physics and cosmology (two modes of inquiry exploring the nature of physical reality) and computing: ${ }^{27}$ a short circuit revealing of the ontological significance of computer time- "If you understand the universe as a giant computer constantly calculating its way through time, it's always easier-less 
resource-intensive-for things to flow forward (cause, then effect) than backward (effect, then cause). This idea is called the "arrow of time." ${ }^{28}$ As Sybille Krämer says, time moves in this imaginary from being "a universal form of our perception or existence" to "a universal form of technological accessibility."29

Even without tachyons to help, the "light cone" ${ }^{30}$ of events in computing can seem to violate causality. In Flash Boys, Michael Lewis describes an interesting move that high-frequency traders in dark pools made. Brad Katsuyama from the Royal Bank of Canada was wondering why when he put in a "buy" order, the shares he wanted to buy (which were listed) evaporated before he could complete the trade-even though he saw the price and the offer in "real time." It gets baroque, but it turns out the reason was that there were several different places to do trades clustered around NY and New Jersey, and some folks had learned how to manipulate millisecond differences in trading in such a way that offers and prices were manipulated before his orders went through. So he wrote an algorithm that deliberately slowed his messages to some of the trading centers so that all his messages arrived at each of the centers at the same time. This produced a realignment between the "ticker tape" prices and offers and his trades. So the ticker tape (central to the temporality of the market since the nineteenth century) ${ }^{31}$ did not show anything real until the delays were brought in — a real "live" market could only be produced in this way. ${ }^{32}$ The role of delay at very high speeds in order to create the appearance of real-time is central to Kittler's canonical essay on time-axis manipulation-he observes that there is no "real-time" analysis, delay is needed in order to assemble the parts ("only that which is switchable is at all") to give the impression of events passing in real time. ${ }^{33}$ This new reality is only buildable because "time exists as quantified and synchronized packets whose size approaches zero." ${ }^{34}$ As Espen Ytreberg remarks in the context of the production of live events on television, the present is an outcome of the manipulation of display. ${ }^{35}$

These time-manipulations, at speeds far surpassing human perception, are real in their consequences: fortunes are lost and won; real-time events are created on television sets. And as David Mills asserts, "Reliable and accurate computer time is necessary for any real-time distributed computer application, which is what much of our public infrastructure has become." ${ }^{36}$ What of the human in this? Well, for one thing, the micro-temporality of the computer can only be supported through the macro-temporality of constant care. ${ }^{37}$ Humans have been in the loop since the earliest days of computing - Babbage envisaged needing them for logarithmic functions (when the machine needed a log function it would ring a bell; if the attendant 
brought the wrong card containing the function, it would just ring louder). ${ }^{3}$ Beverungen and Lange discuss a high-frequency trader matching his circadian rhythm to the cadences of his algorithms - the futures market he traded in was open 23 hours a day; he only slept four hours a night and even then got up every 45 minutes to tend his flock of "algos" (they were pre-programmed to rewrite themselves and interact in complex ways, so one could never be sure what decisions they were making). 39

Where game theoretic economists gave us homo economicus (the person making "rational" choices defined by self-interest), one dream now is of machina economicus, where algorithms working at the speed of light could make rational choices for us ${ }^{40}$; tellingly Parkes and Wellman anoint this "a new species of machine. ${ }^{n 11} \mathrm{~A}$ recent textbook on Blockchain (the technology behind Bitcoin) promulgated the vision that we would need to delegate more than our rational qualities to machines, but also our ethics and our policy; the future would see "a public open distributed ledger with general purpose rational agents (Machina Economicus) running on blockchain, making decisions and interacting with other intelligent autonomous agents on behalf of humans and regulated by code instead of law or paper contracts," and the new technology would "impact every industry including but not limited to finance, government, and media. ${ }^{122}$ By this vision, there would be a whole level of life going on at speeds far exceeding any possible human perception:

One solution might be to introduce intelligent bots or AAs [autonomous agents] or even contracts that are programmed with regulatory logic embedded within them. They are most likely programmed by regulators and law enforcement agencies and live on the blockchain as a means to provide governance and control. 43

The coinage "live" is interesting here; for they will certainly have to be autonomous and adaptive - the comforting myth of their being programmed by humans as unreal as in the case of high-frequency trading: if this were to come to pass, they would need to be designed by algorithms to evolve by circumstance. Larry Lessig, who went from being a contract lawyer to writing about code as law, would surely recognize this world. ${ }^{44}$ In general, it has been claimed, human knowledge and cognition are progressively more marginal in markets. ${ }^{45}$

Where is the real in all this? It is certainly the case that staggering resources are being put into making computers (and the algorithms they spawn) faster. Equally certain, new kinds of events are occurring in the world spawned by new kinds of entities which act using much the same register 
as we have ascribed to intentional agents in the past. If we take ontology as being about what kinds of things the world is peopled with, there are indeed new kinds of entities, operating at new times.

Just as evolutionary theory looks very different if taken at the temporality of the virus or the human (even current attempts to find selection within one human generation - such as lactose tolerance-are necessarily concerned with multiple years ${ }^{46}$ ); so does history look very different if taken at the temporality of these new entities. ${ }^{47}$ The real question is whether it is just the world inside the computer, or if something very different is going on at this new ontological level—will we find, as Jacques Revel observed in the context of microhistory over short time scales, that "the change in the scale of observation revealed not just familiar objects in miniature but different configurations of the social. ${ }^{248}$

\section{Linear Time}

The formal structure of time in a computer is relatively simple: it all comes down to carrying out one instruction per tick of the clock (I am not covering here modifications which partially work around this; they do not make an analytic difference) - the faster the clock ticks, the faster the computer performs. The trouble is that clocks can only tick so fast:

For many years, we could count on processor clock speeds increasing at a steady rate. Physical limitations present a fundamental roadblock to ever-increasing clock speeds, however: because power density increases superlinearly with clock speed, chips run the risk of melting once their clock speeds become high enough. In order to perform more computations per second, therefore, chips are being designed to contain not just one but several processing "cores."49

As we have seen, it really is serial despite the language of parallelism. In a process which might be called descentralization, each set of commands in parallel processing can be converted into a serial set (one darned thing after another):

the memory behaves as if the instructions were executed sequentially according to some global linear order that preserves the individual orders in which each processor issues its own instructions. For dynamic multithreaded computations, which are scheduled onto processors 
automatically by the concurrency platform, the shared memory behaves as if the multithreaded computation's instructions were interleaved to produce a linear order..$^{\circ}$

It is hard work maintaining this remorseless linearity-both the Therac-25 radiation therapy deaths ${ }^{51}$ and the North American Blackout of 2003 were caused by so-called race conditions. These are when time gets out of joint and a new value is posted to a variable when the old value was still needed: "A determinacy race occurs when two logically parallel instructions access the same memory location and at least one of the instructions performs a write." ${ }^{2}$ The difficulty arises because most of the time the program will run well (which makes it very hard to test for), but occasionally the unlikely but logically possible race will occur and prove disastrous. Again, events at the micro level have macro effects.

There is an analogy here with quantum mechanics. At the level of our day-to-day perception (being, as we are, the measure of all things) a Newtonian physics is good enough to go by; at the level of the very small and very fast, it seems as if there is a new set of rules - a different kind of physics. Our generic relationship with computers works pretty well if we assume they work in the same temporality as ours, just scaled down. When it gets interesting is when the nested scaling breaks down-as in a determinacy race-and we need to recognize that time becomes a different thing both sociotechnically (it becomes pure commodity) and socionaturally (it becomes granular, discontinuous - taking on just the property that Kittler hailed as central ${ }^{53}$ ). And the temporalities are consonant-in datestamp format: "The second can be represented to about 500 attoseconds, or about a tenth of the time light takes to pass through an atom." 54 We need that shorter time.

\section{Historical Time}

Computers have come to define new historical epochs - somewhat helter skelter but with a determined drive: "With the invention of bitcoin in 2008 the world was introduced to a new concept that is now likely to revolutionize the whole of society. It's something that has promised to impact every industry including but not limited to finance, government, and media."55 Not bad for an algorithm - the mining of bitcoins is now, alas, no longer "democratic" but under the control of large organizations that can afford the suites of graphics cards that can mine most efficiently. 
Within the computer, all kinds of historical time are reproduced. For the Ethereum blockchain algorithm, the oil companies have reappeared, with two key variables being gasPrice and gasLimit. ${ }^{6}$ In his book on computer time synchronization, David Mills revels in the investing of human historical temporality into the computer. He tells of the need to separate "the "truechimers, whose clocks gloriously tell the truth from the falsetickers, whose clocks lie viciously." ${ }^{57}$ These terms are not his florid invention-they are terms of the art in the field of computer time. All the pageantry of human history can be found at the level of the femtosecond:

Even under peacetime conditions, the truechimers surviving the select algorithm might have somewhat different time offsets due to asymmetric delays and network jitter. Various kinds of cluster and combine algorithms have been found useful to deliver the best time from the unruly bunch. The one used in NTP sorts the offsets by a quality metric, then repeatedly discards the outlier with the worst quality until further discards will not reduce the residual error or until a minimum number of servers remain. The final clock adjustment is computed as a weighted average of the survivors..$^{8}$

This war of clock against clock is needed to prevent stocks from being sold before they are bought or the evening news coming on at midnight. ${ }^{99}$ At stake is nothing less than the principle of cause and effect (it is notable how often the troubling of cause and effect comes up in discussions of computing). Thus, in real-time systems, in order to avoid "anomalous behaviors (e.g., by actions that bypass the system's normal operation and could violate the cause-effect relation by "making holes" in the "light cone" of events, the interactions between the system and the environment must be explicitly take into account." ${ }^{60}$ For Mills, the internet is a growing child, with its development measured in its need for and colonization of ever shorter time intervals—100 microseconds when it was "teething" to a few microseconds in its current "adolescent" state.$^{61}$ He looks forward to the glorious rule, pace E. P. Thompson, of time, work-discipline, and postindustrial capitalism: "The ultimate accuracy can be achieved only when the clock can be disciplined with exquisitely intimate means. In practice, this requires the discipline algorithm, normally implemented in the NTP software, to be implemented in the operating system kernel." Just as we have been disciplined over the past few hundred years out of lives lived by diurnal and seasonal rhythms into lives run on schedule, by calendar, so have our computers undergone their own temporal discipline over the past thirty years. 
We have seen the importance of voting models. It need not be about industrial discipline under democratic values, though. Professor of Cognitive Robotics Murray Shanahan reckons that in superintelligent (meaning beyond human intelligence) AI might become a "willing intellectual slave who never eats or sleeps and wants nothing more than to work" and that this would be "many corporations' idea of the perfect employee, especially if they don't require wages. ${ }^{n 2}$ He does wonder, though, whether they could be treated as slaves with "moral impunity." ${ }^{3}$ In turn, self-reproducing superintelligent machines could "colonize the galaxy." ${ }^{64}$ One popular methodology is the imperialist competitive algorithm, which models "social evolution." ${ }^{\text {" }}$ It involves assimilating colonies, the occasional revolution, and of course intense warfare between empires. So former economic orders don't go away, they get buried in machines operating in temporalities humans cannot directly access. There is an analogy here with the kind of historicity explored by Kathleen Davis. ${ }^{66}$ She points to the cocreation of slavery and colonial power (extended in space) and the myth of the middle ages and feudal order (extended back in time). The computer spawns multiple historical times which we thought were over (ontogeny recapitulating phylogeny). ${ }^{67}$

There is a trope in the history of science that Copernicus left us with an earth occupying no privileged position in the universe, Darwin left humans with no privileged position in time, and Freud left reason with no privileged position in the mind. As early as 1985 , Michael Arbib argued that the computer left humans with no privileged position in society. ${ }^{68}$ Shanahan leaves us with no privileged position in history, arguing that, possibly, real history is about the history of matter gaining complexity. ${ }^{69}$ His position is reminiscent of Sybille Krämer's whether this disappearance of humans from the story was "the intellectual property of a media-technological version of eschatology."70 From this decentered position, it makes a sort of sense that we project onto matter-with a far greater temporal range than our own-the course of (non)human history.

\section{Present (Future/Past)}

Much writing about computers inhabits the future tense-it's as pervasive as the passive voice of policy documents. Babbage concluded of his Analytical Engine in $185^{2}$ that "nothing but teaching the engine to foresee and then to act on that foresight could lead me to the object I desired." ${ }^{11}$

Prediction has been core to the development of computing in the twenty-first century. It often works on a form of neutral hypothesis-it is easiest 
if one assumes that the future is like the present: we are constantly "updating to remain the same," in Wendy Chun's lovely locution, ${ }^{72}$ because the power of, say, recommender systems is to make us cleave to our present profile in the future. The work of a "discriminant" in machine learning (such as credit risk) is based on the assertion that "the future is like the present."73 We are creating a new "temporeality." 74

The colonization of the future by the present is a theme explored by Hartog historiographically: "The present became something immense, invasive, and omnipresent, blocking out any other viewpoint, fabricating on a daily basis the past and the future it needed." ${ }^{75} \mathrm{He}$ ties it to a shift away from the future being seen as holding promise (as in the Victorian age of certaint $y^{76}$ or the French revolutionary fervor for a better society) to the future as being one of threat and risk (environmental degradation, biodiversity loss, and so forth). A common trope in computing holds similar fears. Sometime in the next twenty to forty years, the world as we know it will change radically and frequently not for the better-it depends on how you understand the coming "singularity." Is it a nightmare or an optimistic vision to pose the following: "Between 2020 and 2050: Artificially Intelligent DAOs [distributed autonomous organizations] will prevail on blockchains that will make rational decisions on behalf of humans"?77 It is certainly a world in which "humans have become increasingly irrelevant." ${ }^{78}$

Jürgen Schmidhuber, who sees full artificial intelligence as a simple function of whether or not we can compress time into small enough units in the computer, takes a trope that was created by Ray Kurzweil and recently developed by Geoffrey West. ${ }^{79}$ Counting down from the omega point, he argues that we have witnessed (for West, we require) ever faster rates of breakthrough in modes of life-starting from humans leaving Africa at $2^{9}$ lifetimes, we advance, step by logarithmic step, down to the Greeks inventing democracy and science at $2^{5}$ lifetimes, the Age of Enlightenment at $2^{2}$ lifetimes, PCs at half a lifetime, and the upcoming revolution projected to occur in a quarter of a lifetime. ${ }^{80}$ (Schmidhuber is somewhat tongue-in-cheek here-he does wonder at the end of the paper whether this compelling scale is more a feature of the way we remember-giving more weight to recency - than of the nature of the record.) In this vision we need deal only with ever-shorter futures until the future disappears through machine learning into the unchanging, untheorized singularity.

Similarly, there the past is colonized by the present to the point of disappearance. We can see this through the language of the Internet of Things. Pioneering cybernetician W. Ross Ashby asserted in the 1950s that a well-configured cybernetic system did not need to hold its own memory. 
The world, and the machines used to analyze it, could be constructed in terms of closed Markov chains - meaning that all one ever needed to know to understand the state of either world or machine was to know its present state and the set of rules for its change. Remembering what a former state had been was simply irrelevant. This has been a major temporal metaphor since the Enlightenment - for Laplace, the universe could be uniquely described by a set of first-order differential equations, meaning that knowing the state of the world right now meant that you could predict its future state to any degree of required accuracy.

As Ashby developed his work especially in Design for a Brain, ${ }^{81}$ he made much of a theory of interchangeability, whereby contingent history just did not matter - the past was irrelevant. We might "lose" Hampshire to a devastating accident, but the Britain would auto-regulate and keep functioning in the future pretty much as in the past-Britain was a system with interchangeable parts. Similarly, the death of an individual human just did not mean much in terms of the temporality of the system. Humans are interchangeable with each other and with machines- "really" we are just talking about a set of operations carried out in metronomic fashion.

When Neil Gross, a pragmatist sociologist, wrote a piece in 1999 entitled "The Earth Will Don an Electronic Skin" presaging the Internet of Things, he wrote: "Hundreds of thousands of PCs working in concert have already tackled complex computing problems. In the future, some scientists expect spontaneous computer networks to emerge, forming a 'huge digital creature." ${ }^{82}$ His image was of a Leviathan, matching Hobbes' (the famous frontispiece to whose work pictures a colossus made of humans forming the body of the king/state). The PCs are working in concert—-they are synchronized together. He goes on:

It will use the Internet as a scaffold to support and transmit its sensations. This skin is already being stitched together. It consists of millions of embedded electronic measuring devices: thermostats, pressure gauges, pollution detectors, cameras, microphones, glucose sensors, EKGs, electroencephalographs. These will probe and monitor cities and endangered species, the atmosphere, our ships, highways and fleets of trucks, our conversations, our bodies-even our dreams.

This image of the colossus well exceeds human temporal scales-it exists in the eternal present of the responsive machine. It is a fundamentally new fact about human existence that our human temporality is now that of the sociotechnical world we have created. 
There is a whole new ontological level which works at ever small time scales and yet which can have large-scale effects. Humans drop out of the equations in much the same way as objects drop out of the equations of quantum mechanics. They are not quite the self-contained things we used to imagine: for both, we need new sets of rules to study in fine the operation of the world. We have both constructed physically and constituted socially new temporalities and new understandings of objects that just do not work at the rate of human perception.

\section{Conclusion}

Time is at the center of our political economy_displacing space, which was central to colonial power: the demotion of the meter to the distance per second traveled by light $(1 / 299,792,458)$ is but a symptom of this new form. Network time protocol is enforced in "space, on the seabed ... and on every continent, including Antarctica." ${ }^{\prime 3}$

The control of space was the control of other countries in far-flung empires. But it was already about the control of time. When the oil companies came into Venezuela, they were able to interact beneath the state-in the subsurface-manipulating temporalities that had never explicitly been part of Venezuelan economic life. ${ }^{84}$ This knowledge enabled them to help supercharge the metabolism of the human species by tapping into low-entropy stored solar energy and converting it into commodities and waste at a higher entropy. ${ }^{85}$ Computer time is central here: the Schlumberger company was at the fore of the development of expert systems to deal with microtemporalities (reading seismic waves), which revealed the macro structure of the earth. As Paul Prudence says, "Algorithms can become a Rosetta Stone for the lithic scryer, for if the writing of stones are hieroglyphics for Caillois then the language of algorithms is a demotic script that can bridge the gap between geological noise and intelligible information. ${ }^{\text {" } 66}$

Learning to work temporalities outside of our immediate sensory experience has been central to the working of capitalism. Alfred Sohn-Rethel's assertion that Galilean absolute space and time was basically about the free flow of the commodity form in an ideal, frictionless market gains traction in this context. The faster the money-commodity-money cycle, the more surplus value can be created-in other words, the more we can work at higher temporalities, the better for the system.

The remorseless, rapid ticking of the computer clock enables control at a wide range of temporal scales. The rapid ticking of the truechimers is 
a source simultaneously of the speed and acceleration we experience in the present and the extension of the present into the immediate past and future, creating Hartog's "monstrous present." 87 There is no historical time as modelled by historiographers on the one side and computer time on the other. At all temporal scales they interpenetrate. This happens at the scale of the longue durée: the computer grew up with and is modeled on factory production as industrialization took command of world economies (from the pillaging of natural resources by the colonizing powers to the organization of manufacturing hubs in the developed world). The event of the short durée is increasingly mediated by machine learning. And at the very shortest scales - the femtosecond - a variety of historical times play out.

We saw above Revel's assertion that scaling down temporally produced new configurations of the social and the political. ${ }^{88}$ In computer time, even while all of these temporal scales interpenetrate, different configurations are being developed. But they are made of the same stuff as standard historical time. Inside computers, we find colonialism, slavery, resource control, and the manufactured event beloved of media theory-as well as geological and evolutionary time. The technological here is not different from other spheres of existence; it is fully coeval with them.

This can only really make sense if we see that the sociotechnical and the socionatural work together. There is no nature "out there" for the computer - we understand nature through its workings at the same time as we model the computer on that understanding.

Life at the femtosecond is consequential for our ways of being in, apprehending, and acting in the world. Real-time is ineluctably real time, and for us real time is now real-time.

\section{Notes}

1. François Hartog, Régimes d'historicité: Présentisme et experiences (Paris: Seuil, 2003), 120. "Through speed, the present transforms itself into eternity" (my translation).

2. $\quad$ Craig Callender What Makes Time Special (Oxford and New York: Oxford University Press, 2017), 156.

3. I'm Sorry I Haven't a Clue, Season 70, Episode 2.

4. Charles Babbage, "On a Method of Expressing by Signs the Action of Machinery," Philosophical Transactions of the Royal Society of London 116, no. 1-3 (1826), 250-265.

5. Charles Babbage, Passages from the Life of a Philosopher, ed. Martin Campbell-Kelly (New Brunswick, NJ: Rutgers University Press, 1994 [1864]), 94. 
6. Babbage, Passages, 90.

7. See Charles Lyell, The Principles of Geology, vol. 1 (Chicago: University of Chicago Press, 1990 [1830]).

8. Charles Babbage, The Ninth Bridgewater Treatise: A Fragment, 2nd ed. (London: John Murray, 1838).

9. This temporal collapse is perhaps pointed to by Kittler's use of "a screaming comes across the sky" (16) - the opening and closing lines of Gravity's Rainbow, encapsulating the extensive time of the novel into the moment of the dropping of a rocket.

10. Thomas H. Cormen, Charles E. Leiserson, Ronald L. Rivest, and Clifford Stein, Introduction to Algorithms, 3rd ed. (Cambridge, MA: MIT Press, 2009), 774.

11. David Deutsch, The Fabric of Reality (London: Penguin, 1997), 155.

12. Deutsch, Fabric of Reality, 200, 196-7.

13. I borrow this felicitous phrase from Robert F. Service, "Time's Romance of the Decimal Point," Science 306, no. 5700 (November 19, 2004): 1310-1311.

14. AI Impacts, https://aiimpacts.org/brain-performance-in-flops/, accessed November 25, 2019. Kurzweil gives $10^{16}$.

15. "Messy emulation" is just doing neuron-for-neuron mapping; other forms would develop new kinds of intelligence. (It is surprising that such a limited view of the workings of the brain - neuron firing - persists in computer science, as well as the willful ignorance of the distributed, embodied nature of cognition.)

16. Murray Shanahan, The Technological Singularity (Cambridge, MA: MIT Press, 2015), 152-3.

17. Shanahan, Technological Singularity, 157.

18. Dow Schulman, xxx.

19. Armin Beverungen and Ann-Christine Lange, "Cognition in High-Frequency Trading: The Costs of Consciousness and the Limits of Automation," Theory, Culture \& Society 35, no. 6 (2018): 80.

20. Timothy Mitchell, Carbon Democracy: Political Power in the Age of Oil (London: Verso, 2011), 15 .

21. Ian Osborne and Daniel Clery, "Higher Standards," Science 306, no. 5700 (November 19, 2004): 1307.

22. Gary Genosko and Paul Hegarty, "Where Has Become of Time? Temporal Smearing and Media Theory," SemiotiX, https://semioticon.com/semiotix/2018/o3/where-has-become-of-time-temporal-smearing-and-mediatheory/.

23. Simon Kemp, "Digital in 2018: World's Internet Users Pass the 4 Billion Mark," We Are Social, January 20, 2018, https://wearesocial.com/ blog/2018/01/global-digital-report-2018. This is spread over 4 billion users, and interesting more for the order of magnitude rather than as a realistic assessment.

24. Quoted in Jussi Parikka, "Introduction," in Wolfgang Ernst, Digital Memory and the Archive, ed. Jussi Parikka (Minneapolis: University of Minnesota Press, 2014), 15. 
25. Michael Reilly, "High-Frequency Trading is Nearing the Ultimate Speed Limit," MIT Technology Review, August 9, 2016, https://www.technologyreview. com/s/6o2135/high-frequency-trading-is-nearing-the-ultimate-speed-limit/.

26. Reilly, "High-Frequency Trading."

27. See, for example: Rafi Letzter, "How Quantum Computers Could Kill the Arrow of Time," Live Science, July 27, 2018, https://www.livescience. com/63182-quantum-computer-reverse-arrow-time.html.

28. Letzter, "Quantum Computers."

29. Sybille Krämer, "The Cultural Techniques of Time Axis Manipulation: on Friedrich Kittler's Conception of Media," Theory, Culture \& Society, 23, no. 7-8 (2006): 93-109

30. Fabio A. Schreiber, "Is Time a Real Time? An Overview of Time Ontology in Informatics," in Wolfgang A. Halang and Alexander D. Stoyeno (eds.), Real Time Computing, NATO ASI Series, Series F: Computer and Information Systems 127 (1994): 294.

31. Alex Preda, "Socio-technical Agency in Financial Markets: The Case of the Stock Ticker," Social Studies of Science 36, no. 5 (2006): 753-782. Speed has been central to the market since at least the late eighteenth century-Chartier $(\mathrm{xxx})$ points to five issues of a paper being put out daily to describe market changes. We often forget this time compression from pre-digital days-in London in the early nineteenth century there would be four or five postal deliveries a day_making the service more like email than the more recent instantiations we tend to project onto the past (under the retronym "snail mail").

32. Michael Lewis, Flashboys: Cracking the Money Code (London: Allen Lane, Penguin. 2014).

33. Friedrich Kittler, "Real Time Analysis, Time Axis Manipulation," Cultural Politics 13, no. 1 (2017), 1-17.

34. Kittler, "Real Time Analysis," 10.

35. Espen Ytreberg.

36. David L. Mills, Computer Network Time Synchronization: The Network Time Protocol on Earth and in Space, 2nd ed. (Boca Raton, FL: CRC Press, 2016), xxiii.

37. See Gabriele Schabacher in this volume.

38. Babbage, Passages, 90.

39. Beverungen and Lange, "Cognition in High-Frequency Trading," 88-89.

40. Martin Prause, "On the Trail of Machina Economicus," Info Insights, 2017.

41. David C. Parkes and Michael P. Wellman, "Economic Reasoning and Artificial Intelligence," Science 349, no. 6245 (July 17, 2015): 267-272. doi:10.1126/ science.aaa8403.

42. Imran Bashir, Mastering Blockchain: Distributed Ledgers Decentralization and Smart Contracts Explained (Birmingham-Mumbai: Packt, 2018), 1.

43. Bashir, Mastering Blockchain, 475 .

44. Lawrence Lessig, "Law regulating code regulating law," Loyola University Chicago Law Journal 35 (2003): 1-14.

45. Beverungen and Lange, "Cognition in High-Frequency Trading," 80. 
46. Elizabeth Pennisi, "Humans Are Still Evolving-And We Can Watch It Happen," Science, May 17, 2016, https://www.sciencemag.org/news/2016/05/ humans-are-still-evolving-and-we-can-watch-it-happen.

47. Thanks to Bodhisattva Chattopadhyay for this observation. See Bodhisattva Chattopadhyay and Geoffrey C. Bowker, "Ant Network Theory," NatureCulture 5 (2019): 26-49.

48. Jacques Revel, introduction to Histories: French Constructions of the Past, ed. Jacques Revel and Lynn Hunt, trans. Arthur Goldhammer (New York: New Press, 1995), 46.

49. Cormen et al., Introduction to Algorithms, 10.

50. Cormen et al., Introduction to Algorithms, 779.

51. Nancy G. Leveson and Clark S. Turner, "An Investigation of the Therac-25 Accidents," Computer 26, no. 7 (1993): 18-41.

52. Cormen et al., Introduction to Algorithms, 788.

53. Kittler, Discourse Networks 1800 / 19oo, trans. Michael Metteer (Stanford, CA: Stanford University Press, 1992).

54. Mills, Computer Network Time Synchronization, 18.

55. Bashir, Mastering Blockchain, 1.

56. Bashir, Mastering Blockchain, 216.

57. Mills, Computer Network Time Synchronization, 7.

58. Mills, Computer Network Time Synchronization, 8.

59. Mills, Computer Network Time Synchronization, 10.

6o. Fabio A. Schreiber, "Is Time a Real Time? An Overview of Time Ontology in Informatics" in Wolfgang A. Halang and Alexander D. Stoyeno (eds.), Real Time Computing, NATO ASI Series, Series F: Computer and Information Systems 127 (1994): 283-307.

294.

61. Schreiber, "Is Time a Real Time?," 9 .

62. Murray Shanahan, The Technological Singularity (Cambridge, MA: MIT

Press, 2015), 93. See E. P. Thompson, "Time, Work-Discipline, and Industrial Capitalism," Past \& Present 38 (1967): 56-97.

63. Shanahan, The Technological Singularity, 153.

64. Shanahan, The Technological Singularity, 157.

65. https://en.wikipedia.org/wiki/Imperialist_competitive_algorithm. Accessed December 21, 2018.

66. Kathleen Davis, Periodization and Sovereignty: How Ideas of Feudalism and Secularization Govern the Politics of Time (Philadelphia: University of Pennsylvania Press, 2008), 42-50.

67. Ernst Haeckel, who was so influential in establishing the tree metaphor for biological descent also gave us the resounding tocsin "ontogeny recapitulates phylogeny." The two are closely linked when taken in terms of information organization and access in computer science terms; and in terms of historiographical analysis in the nineteenth century in humanist terms.

68. Michael A. Arbib, "On Being Human in the Computer Age," in Impacts of Artificial Intelligence, ed. Robert Trappl (Amsterdam: Elsevier, 1985). 
69. Shanahan, The Technological Singularity, xxii-xxiii.

70. Krämer, "The Cultural Techniques of Time Axis Manipulation," 107.

71. Babbage, Passages, 86.

72. Wendy Chun, Updating to Remain the Same (Cambridge, MA: MIT Press, 2016), 79-85.

73. Ethem Alpaydin, Introduction to Machine Learning, 3rd ed. (Cambridge, MA: MIT Press, 2014).

74. Jussi Parikka, “The Underpinning Time: From Digital Memory to Network Microtemporality," in Digital Memory Studies: Media Pasts in Transition, ed. Andrew Hoskins (New York: Routledge, 2018), 156-172.

75. François Hartog, Regimes of Historicity: Presentism and Experiences of Time (New York: Columbia University Press, 2015), 185.

76. Asa Briggs, A Social History of England (New York: Viking, 1984).

77. Bashir, Mastering Blockchain, 475 .

78. Kittler, "Real Time Analysis," 13.

79. Ray Kurzweil, The Singularity Is Near (New York: Penguin, 2005). Geoffrey West, Scale: The Universal Laws of Growth, Innovation, Sustainability, and the Pace of life, in Organisms, Cities, Economies, and Companies (New York: Penguin, 2017).

8o. Jürgen Schmidhuber, "New Millennnium AI and the Convergence of History," ArXiv, June, 29, 2006.

81. W. Ross Ashby, Design for a Brain (London: Chapman \& Hall, 1954).

82. Neil Gross, "The Earth Will Don an Electronic Skin," Bloomberg, August 30, 1999, https://www.bloomberg.com/news/articles/1999-08-29/14-the-earthwill-don-an-electronic-skin.

83. Mills, Computer Network Time Synchronization, 23.

84. Geoffrey C. Bowker, Science on the Run: Information Management and Industrial Geophysics at Schlumberger, 1920-1940 (Cambridge, MA: MIT Press, 1994).

85. Alf Hornborg, "Machine Fetishism, Value, and the Image of Unlimited Good: Towards a Thermodynamics of Imperialism" in Man, New Series, Vol. 27, No. 1 (Mar., 1992), pp. 1-18.

86. Paul Prudence, "The Algorithmic Writing of Stones: A Cybernetics of Geology," SubStance 47, no. 2 (2018): 71-83: 82.

87. I borrow this phrase from Helge Jordheim, personal communication.

88. Revel, Histories, 206.

\section{About the Author}

Geoffrey C. Bowker is Donald Bren Chair at the School of Information and Computer Sciences, University of California at Irvine. Together with Leigh Star he wrote Sorting Things Out: Classification and its Consequences. His most recent book is Memory Practices in the Sciences. 


\title{
7. Artificial Intelligence and the Temporality of Machine Images
}

\author{
Andrew R. Johnston
}

\begin{abstract}
DeepMind, a recent artificial intelligence technology created at Google, references in its name the relationship in AI between models of cognition used in this technology's development and its new deep learning algorithms. This chapter shows how AI researchers have been attempting to reproduce applied learning strategies in humans but have difficulty accessing and visualizing the computational actions of their algorithms. Google created an interface for engaging with computational temporalities through the production of visual animations based on DeepMind machine-learning test runs of Atari 2600 video games. These machine play animations bear the traces of not only DeepMind's operations, but also of contemporary shifts in how computational time is accessed and understood.
\end{abstract}

Keywords: artificial intelligence, animation, digital media, video games, technology, history

DeepMind, a recent artificial intelligence technology created at Google, references in its name the relationship in AI between models of cognition used in this technology's development and the deep learning algorithms that make up its layers of nonlinear processing units. With DeepMind Google has been attempting to reproduce patterns of visually based reinforcement learning strategies observed in human subjects through the use of big data and probability scenarios in neural networks. Like other AI that were created and used in speech recognition or machine translation applications, researchers at Google encountered two problems: 1) how to model reactive sensory systems and 2) how to visualize and access

Volmar, A. and K. Stine (eds.), Media Infrastructures and the Politics of Digital Time: Essays on Hardwired Temporalities. Amsterdam: Amsterdam University Press, 2021 DOI 10.5117/9789463727426_CHO7 
their mechanics when the systems operate below thresholds of human sensory perception and cognition. The second question is one endemic to contemporary computational processes and sets of big data generally, whose weighty flows of indeterminate scales of information are born out through representational tropes or descriptions that refer to their inaccessibility. Researchers managed both problems by designing an AI system that could train to win emulated Atari 2600 video games such as Qbert, Space Invaders, Breakout, and 46 others, each played 600 times in order to visualize the mechanics of the AI. The gameplay videos, or machine runs, of these training sessions offer representational data points for researchers who map how reinforcement learning strategies are employed over the course of various gameplay scenarios, revealing in visual space how the AI adapts to different contexts and evaluation criteria through operations in time that are otherwise inaccessible.

This out of reach character of digital technologies is commonplace. Materialist analyses of digital media, like the ones included in this volume, have brought attention to the disjuncture of temporalities present in contemporary technological systems and regimes of experience. Running at speeds faster than that which sensation can detect and through machinations outside the purview of consciousness, digital technology seems to break traditions of technological synchronization that match human experience to the temporal actions of machines. Descriptions of this historically antecedent organization of time and technics can be seen in the work of Mary Ann Doane, who, following E.P. Thompson, argues that a nineteenth century reorganization of time was engendered by industrial revolution machines and clocks that enabled it to be measured and divided. This also externalized temporality as a "surface phenomenon" that individuals attempted to capture through mediated representations. ${ }^{1}$ That chase was precisely what Paul Virilio worried over, as he envisioned how the mutual accelerations of technology and understandings of time could erode the limits of human history, knowledge claims, and order. $^{2}$

The link between human sensation and machinic temporality is what scholars such as Mark Hansen and Wolfgang Ernst argue has been severed by contemporary digital technologies. Ernst emphasizes how digital technologies materially instantiate a time outside of consciousness through the calculation and flow of signals so that even digital "storage is nothing but a limit value of transfer ... storage is a transfer across a temporal distance." This articulation of time and space is microscopic in scale and employed by digital media for the abstractions of signal transmissions, which allow for later formal actions that are made available to the senses by these objects. 
Hansen makes a similar point, arguing that twenty-first-century media, or digital objects and network infrastructures, "operate predominantly, if not almost entirely, outside the scope of human modes of awareness (consciousness, attention, sense perception, etc.)."4 The resulting human experiences with these media and their environments are therefore only indirect relative to their machinations; since one cannot witness their signal pulses, to experience them is to be at a remove from their technical work. Hansen argues that because of this arrangement contemporary digital media has a predictive relation to our bodies in a feed forward architecture "that literally mediate the data of causal efficacy (as measured, calculated, and analyzed by twenty-first-century media) for future consciousness to factor into its activity-to-come." Since digital technologies are built upon indirect engagements with their inner workings, their signal transmissions are structured through predictions of future action in a race with consciousness and experience to produce illusions of interactivity.

All these epistemological formulations are rooted in contemporary digital media. And for good reason, since digital technology used for computer graphics or other applications in the 1960 s and 1970s was notoriously slow relative to analog computers and often relied on paper storage. ${ }^{6}$ But the above authors also emphasize how the operations of contemporary digital media are at such a remove that they appear opaque, a black boxing of technology that Ernst, as well as Bruno Latour, warn against in understanding material renderings of experience brought about by shifting technical regimes. ${ }^{7}$ The seeming opacity of digital media is certainly a cultural phenomenon that generates anxiety, often manifest in new genres or mediated by horror narratives, arguments persuasively made by scholars such as James J. Hodge, Shane Denson, and Adam Hart. ${ }^{8}$ That said, this perspective is not totalizing and, as the strange periodizing of Hansen and Ernst reveal, is a shifting process born of multiplicity and contradiction rather than singularity and uniformity. As Ernst emphasizes, "a media-archaeological view of the temporal modes of media almost inevitably leads to a critique of the totalizing collective singularity of 'time' itself."9

To perform this media archeological analysis is to open the black boxes of contemporary digital technologies that can appear so opaque. Doing so performs an epistemological reverse engineering that makes manifest the impact of technologies in the production of knowledge through their mechanisms of sensual and information presence. ${ }^{10}$ And it also clarifies the contours of that epistemology and mediations of temporal experience. While the phenomenal actions of computational media may index obscurity, they also point towards and create new arrangements 
and networks of activity. Such changes may induce anxiety in cultural narratives, a reaction found during other periods of shifting temporalities born of technological change, exemplified in Alvin Toffler's 1970 book Future Shock. ${ }^{11}$ But as past studies of these moments have shown, such transitions are often fractured with multiple layers of technicity interwoven with one another to both manage these changes and develop use strategies. ${ }^{12}$ In this light the machine run videos of DeepMind's Atari play have particular significance, since they are not created as a formal articulation that gestures towards an unknowable obscurity, but instead attempts to quantify and abstract the temporal and computational actions of the AI for later modification. In Google's published work, the abstract neural network of DeepMind is displayed with corresponding screenshots from gameplay to provide illustrations of what they argue is machine learning. These gameplay animations index machine action and are a means through which Google can refine and develop computational deep learning. Doing so, I argue, positions animation as an epistemic object and tool to make nonhuman cognition and time sensually present. Functioning as an investigatory device, it maintains the aesthetic wonder that animation is often associated with, but simultaneously reveals different technical operations buried in the AI system that produce these articulations. ${ }^{13}$ These unseen microtemporal actions are animations unto themselves, since the system analyzes, abstracts, and then synthesizes images into taxonomic frameworks or movements acted upon in a different layer within its feed forward architecture. Though operating at different scales of time, the qualities of technical abstraction and synthesis in other animations are still present, though addressed for a system whose visual syntheses have been modeled from human perception and that we cannot see. But these actions can also become the means through which phenomenal images are generated that then makes sensually present information for human users, a secondary animation layered onto the others. I argue that together this stack of animation produces the interface for working with and manipulating networked infrastructures of AI, an interweaving of what Ernst, quoting Gilbert Simondon, calls different levels of temporal knowledge: "a micro-level of physical and techno-physical processes, [and] a meso-level of psychic-cognitive processes." ${ }^{{ }^{114}}$ Layering them onto one another both enables speculative knowledge about the AI system's temporal modulations and about our relation to these new actions of time. The remainder of this essay will work through and open this AI interface, moving up and down its stacks to reveal animation's role in the diverging temporalities that mark these technologies and this moment. 
AI emerged as a field in the 1950s out of cybernetics and one of its earlier and most long-lasting research areas was visual pattern classification and the development of machines that would have the ability to detect predetermined objects within given fields. Though operating in fits and starts over several decades, this area of AI has recently become a center of inquiry, in part due to the accelerated growth of graphics processing units used in feed forward neural networks created after what many call an AI winter in research during the 1970s. ${ }^{15}$ Unlike other AI information oriented platforms, DeepMind has action-oriented goals achieved through rewards defined within specific environments. It is designed to create software agents that "take actions in an environment so as to maximize some notion of cumulative reward." ${ }^{\prime 6}$ Taken as a whole, this is a feedback system that explores and modifies the agent's environment and like earlier AI systems uses behaviorist psychology frameworks. But its modeling of the ways individuals solve problems through feedback mechanisms is put in conjunction with a hierarchical sorting and categorization of sensory data. Previous AI systems were built around applications set in motion by particular stimuli. IBM's Watson, for instance, is a natural language and information retrieval system, whose functions would be triggered by spoken questions. ${ }^{17}$ Because of this, the system does not explore or test its environment through sensory data, but instead operates through an if-then Boolean logic so that if particular inputs exist, then a functioning action within the platform will result. Using a behavior tree for executed actions, the platform is dependent upon language, accent, and the information database accessible to it.

The exploration of an environment through visual data was still the most prized area of AI research and much of its success has emerged from investigations into vision performed by Nobel prize winning neurobiologists David Hubel and Torsten Wiesel. Beginning in 1958 Hubel and Wiesel traced the mechanisms of action within the visual cortex and showed how the brain processes visual information from the retina through a columnar architecture filled with simple and complex cells, each of which is sensitive to particular visual features for specific parts of the visual field. ${ }^{18}$ In their experiments they determined how the visual cortex of a cat, and later other animals, uses simple nerve cells to locate edges or borders of contrast along with other formal features and that the cortex employs complex nerve cells to combine input from simple cells to gauge motion and other actions. Finally developing a topographical map of the visual cortex, Hubel and Wiesel showed how different neurons from the retina operate as inputs to produce binocular perception and the visual pathway of information in the brain. ${ }^{19}$ 
Importantly, Hubel and Wiesel's experiments also illustrated the feed forward mechanisms through which visual perception functions, which rather than having a singular feedback loop for input and outputs, nests multiple layers of inputs and outputs that don't cycle or loop back to each other but move in a linear fashion. Fundamentally, the nodes in this system constantly evaluate or categorize the input they receive, each making slight transformations before outputting to the next node in a unidirectional manner through time. This design became the model for large computational feed forward networks, which were successfully employed to analyze and categorize images by computer scientists like Yann LeCun in the late 1980s. He developed convolutional neural networks that had many filter layers for the categorization and identification of image features, enabling the system to work through the logic and pattern differentiation problems that plagued AI image research in the past. This requires using Hubel and Wiesel's analysis of edge detection for images but in a large enough network that the subsampling of images continues until the system can accurately identify objects. Breaking the images up many times over, the network races to abstract the image, compare those pieces to others it has analyzed before, and then categorize and take action on that decision. Citing the neurobiologists as a precedent, LeCun describes how this convolutional neural network can be used for handwriting and optical character recognition machine learning, each of which has been employed by the US postal service and several banking institutions. ${ }^{20}$ Google's DeepMind team references and builds from LeCun's model for "exploiting the local spatial correlations present in images" in order to take into account viewpoints that the AI employs while exploring visual space. ${ }^{21}$ Once again, for DeepMind, the AI does not just seek particular data from sensory inputs, but attempts to organize all the information presented to its inputs within a specified domain of action. It explores but categorizes according to specific criteria. Like other contemporary AI systems, DeepMind is a sophisticated bot technology and is an object categorization machine using a feed forward network agent. All the visual information presented to the AI is sorted as quickly as it can through its matrix of subsampling nodes in order to then provide actionable information within the machine's environment.

For those designing AI, the speed of this sorting is the problem. Experiments with machines that could perform these actions took place almost immediately after Hubel and Wiesel's experiments. In the late 1950s Frank Rosenblatt created a pattern classification algorithm called a perceptron that attempted to categorize visual images. Put simply, the perceptron would 
evaluate image information presented to it and determine, through if-then logical statements, whether or not the image had features of a preassigned category. By utilizing multiple inputted weights, or parts of an image, the algorithm could examine the image several times to compare these weighted values to one another in the overall assessment of the image as belonging or not belonging to the pre-assigned category. After a number of tries, certain inputs are deemed more important than others in the image for determining whether or not it belongs to a category, such as "cat pictures." ${ }^{22}$ At the time, the adaptive quality of the algorithm's operations produced massive speculation about the future of AI in the popular press, especially after Rosenblatt created an analog computer for the perceptron that employed 400 photocells for inputs whose resistance parameters were controlled by motors. ${ }^{23}$ Thinking he had successfully determined whether a tank was in a photograph or not, an application sought after by the U.S. military, Rosenblatt postulated that his machine had "original ideas" and that "as a concept, it would seem that the perceptron has established, beyond doubt, the feasibility and principle of nonhuman systems which may embody human cognitive functions." ${ }^{24}$ This fantasy, however, was short-lived since Rosenblatt was actually guessing at what his machine was classifying, learning later that the perceptron was not identifying a tank in photographs, but rather the time of day. ${ }^{25}$ For Rosenblatt, not having an interface through which he could make phenomenal the microtemporal actions of the machine resulted in not knowing what the machine could or would do, a blindness to both its rhythms of time and its output of information.

Animation solves this problem by both completing a visualized feedback loop for researchers and projecting fantasies of autonomy onto its actions in an environment. But the question of reinforcement learning and its relation to mediations of animation loomed large for Google's research team, since DeepMind both responds to and changes the environmental conditions through which it receives sensory information. In short, Google wanted to display an interface of time through animation so it could show consequences of computational temporalities and their effect on a phenomenal world. In attempting to develop this behaviorist project, Google isolated the visual input available for the system and its actionable output. Using emulations of the Atari 2600 games, they coded DeepMind's sensory inputs to be an $84 \times 84$ pixel gameplay grid and game scores, the latter of which would function as evaluation criteria. Structuring the inputs this way not only isolates ideas of reinforcement learning and processing to visual systems, but also limits specific visual operations, namely motion 
detection. Google wanted to develop an AI algorithm in DeepMind that could be used on large neural networks and therefore isolated various parameters of the system at its genesis. Emphasizing a particular action in reinforcement learning, DeepMind excels when encountering puzzles or obstacles that require a measured degree of planning. As Google explains, in Breakout "the agent learns the optimal strategy, which is to first dig a tunnel around the side of the wall allowing the ball to be sent around the back to destroy a large number of blocks" but also admits that "games demanding more temporally extended planning strategies still constitute a major challenge." ${ }^{26}$ Throughout their description of DeepMind it becomes clearer as to why Google selected Atari 2600 games. Not only is the game environment limited spatially and temporally, but the Atari's sprites are also abstract objects with clear figure/ground relations whose spatial correlations require less processing than other platforms. Such reductions focalize the action of the AI while also conflating the rules or logics of each game with the mechanics of the system, an operation that Stephanie Boluk and Patrick LeMieux identify as prevalent within video games. ${ }^{27}$ To be clear, it is the mechanics of the specific game that DeepMind categorizes, since the system cannot abstract the principles of gameplay from one game to another, but instead must begin again and learn the design logics and operations of each game from scratch. The researchers functionally bar the AI from accessing the code and other algorithmic functions that produce the Atari games, a more efficient means through which the neural network could learn the inputs and outputs associated with this gameplay. Instead, they develop an artificial barrier that attempts to mimic a naive player, a sort of DeepMind noob, whose ultimate precociousness is revealed in a dazzling technological display. It is a brilliant marketing move, but also shows how the $\mathrm{AI}$ is learning the mechanics available to objects in the game more than learning how to "play." Google's conflation, like the one Boluk and LeMieux identifies, of video game logic with object mechanics, nonetheless produces a projection of mastery onto the machine as it sorts through various values, actions, and temporal relations over the 600 iterations of play for each Atari game. Since the AI operates through conditions of gameplay dependent upon display projections, the resulting actions run as animations and visually mark the system's performance through the game's challenges.

As such, the animations that make present the operations of the AI exist within a well-known genre that performs a similar function: tool-assisted speed runs. These are programs that exploit the serial interface of emulated video games to reveal the most efficient combination of inputs into the 
game to finish or "win" it. Through an almost linear editing of possible commands within the game's interface, a recorded animation of action emerges and makes phenomenal the operations of the software. Creating an AI interface like this makes familiar both unseen temporalities of the system as well as the aesthetic and informational form of its output for people familiar with the Atari games. The animations make relatable the AI's operations while acutely marking its differences from human users. Though competitive speed runs performed by human players have been popular within gaming communities for some time, the contrast of these with tool-assisted speed runs, as LeMieux explains, more generally "reveals the alienating effects of digital seriality and dramatizes the distinction between human and machine scales of temporality. ${ }^{28}$ With DeepMind, the alienation builds over time since the spectacular failure of the $\mathrm{AI}$ is put on display over the first hundred iterations of gameplay. By limiting the AI's inputs to the screen and score, Google blinds DeepMind to the serial interface of the Atari games, but simultaneously creates visual access to DeepMind's processing operations for researchers. In this way, the machine run animations function as a heuristic tool within the laboratory, standing in not only for ideal human cognition in reinforcement learning scenarios, but modulations of time as well. Google's comparison of gameplay between the $\mathrm{AI}$ and professional game testers emphasize this point, showing how in games like Road Runner, Star Gunner, or Assault, DeepMind operates at or above what it characterizes as "human-level" gameplay, boasting that it "performs at a level that is broadly comparable with or superior to a professional human games tester ... in the majority of games." ${ }^{29}$ Thus, through DeepMind's limited ocular mimicry to analyze its reinforcement learning architecture, the resulting animations bridge forms of human and nonhuman subjectivity by manifesting phenomenal, aesthetic action that both subjects could produce.

Again, without losing its aesthetic contours, animation in this context becomes an instrument of speculation, or what historians of science describe as an epistemic object: an experimental form characterized by indeterminacy within a historical field, but whose instantiations mark patterns of emerging knowledge and categories. Karin Knorr Cetina calls them "objects of knowledge [that] appear to have the capacity to unfold indefinitely ... like open drawers filled with folders extending indefinitely" since their technical composition is in a constant state of change..$^{30}$ Epistemic objects' changes speak to historic patterns that are dependent upon knowledge formations. Like Lisa Gitelman's examination of documents as having a know-show function, that is, of having "the kind of knowing that is wrapped up with 
showing, and showing wrapped up with knowing," animation here works in the same way: as a means of knowing the temporal modulations buried in the AI's operations, and showing the ways in which these machinic temporalities interface with human cognition and sensation. ${ }^{31}$ No longer printing results like computational outputs of the past, $\mathrm{AI}$ and contemporary computer science research uses animation to index and explore interfaces of time. By making sensually apparent the temporal vectors of processing in DeepMind and pitting those against familiar logics of time in game mechanics, Google manifests a laboratory of AI time, acknowledging that as they develop their algorithms, they will turn to games with "more temporally extended planning strategies."

Positioning animation as tool of knowledge production stacked into an interface with multiple directions of address aimed at nonhuman and human actors punctuates its importance in contemporary digital culture. As Hodge emphasizes relative to the historicity of digital media, "animation captures the time-based volatility of digital media" and reveals forms of historical experience constituted through digital technology's obscured articulations of time. ${ }^{32}$ Similarly, the stacks of animation in AI constellates another point in animation's history of the ways in which humans and nonhumans are suffused or intertwined in its constitution, even when seemingly separate from a distance as in the animations of DeepMind's machine runs. Google's animations serve as a means of assessment for DeepMind, a practice which, as they allude to above, has continued in the development of this AI into new gameplay arenas and platforms, such as Starcraft II. ${ }^{33}$ Inviting professional Starcraft II game players to compete against DeepMind, the uncertainty and anxiety over the system's refined iterations is captured not only by the screen recordings of gameplay, but also by reaction shots of DeepMind's development team. Functioning as an epistemological agent, animation for these researchers offers the best means through which traces of the algorithmic functions of sorting and categorizing can be found. It is a communicative medium that indexes an action rhetorically compared to human intelligence and projected as being autonomous, or seemingly alive. This sense of independent action that these machine runs take, and that so much visually based AI has, is a legacy of equivalences of sight with mastery and intelligence, a story often rehearsed and recently challenged by many. ${ }^{34}$ As Bernard Geoghegan illustrates, by the mid-twentieth century this visual emphasis had become fully integrated into both cybernetics and the development of computer visualization systems. ${ }^{35}$ This led to the development of AI that could, in the popular press, make claims to possessing forms of intelligent learning because of actions built 
around the identification, categorization, and analysis of objects within puzzle environments.

Visualizing these actions and making them seen through an interface was equally as important. All the projections of intelligence and senses of life onto DeepMind's animations are not simply bound to the moving images, but also to the technical artifacts that generate them, whose mechanisms seemingly contain an energy that produces actions. By creating a phenomenal action of DeepMind's reinforcement learning processes, Google's research team both employ and index an articulation of animation defined through its ability to make visible modulations of time within contemporary digital media. Its stacks contain prisms of time, with multiple addresses, but these technical articulations make intelligible digital networks while projecting fantasies of agency onto them. The wonder of animation, its projection of movement that theorists like Sergei Eisenstein and Alan Cholodenko have characterized as lifelike, becomes a means of making visible algorithmic forms of time to project notions of intelligent action that can be understood across human and nonhuman subjects.

\section{Notes}

1. Mary Ann Doane, The Emergence of Cinematic Time: Modernity, Contingency, the Archive (Cambridge, MA: Harvard University Press, 2002), 9.

2. Paul Virilio, Politics of the Very Worst, trans. Michael Cavaliere, ed. Sylvère Lotringer (Los Angeles: Semiotext(e), 1999). Grant Bollmer provides an excellent analysis of Virilio's ideas relative to nineteenth century technics in Grant Bollmer, Materialist Media Theory: An Introduction (New York: Bloomsbury, 2019).

3. Wolfgang Ernst, Digital Memory and the Archive, ed. Jussi Parikka (Minneapolis: University of Minnesota, 2013), 100.

4. Mark B. N. Hansen, Feed-Forward: On the Future of Twenty-First-Century Media (Chicago: University of Chicago Press, 2015), 5.

5. Hansen, Feed-Forward, 58 .

6. See Matthew G. Kirschenbaum, Mechanisms: New Media and the Forensic Imagination (Cambridge, MA: MIT Press, 2008); Nick Montfort, "Continuous Paper: The Early Materiality and Workings of Electronic Literature," paper presented at the Modern Language Association conference, Philadelphia, PA, December 2004, http://nickm.com/writing/essays/continuous_paper_mla.html; Tung-Hui Hu, A Prehistory of the Cloud (Cambridge, MA: MIT Press, 2015); Andrew R. Johnston, "Models of Code and the Digital Architecture of Time," Discourse: Journal for Theoretical Studies in Media and Culture 37, no. 3 (Fall 2015): 221-246. 
7. $\quad$ See Wolfgang Ernst, "Media Archaeology: Method and Machine versus History and Narrative of Media," in Media Archaeology: Approaches, Applications, and Implications, eds. Erkki Huhtamo and Jussi Parikka (Berkeley: University of California Press, 2011), 239-255; Bruno Latour, Pandora's Hope: Essays on the Reality of Science Studies (Cambridge, MA: Harvard University Press, 1999).

8. See James J. Hodge, Sensations of History: Animation and New Media Art (Minneapolis: University of Minnesota Press, 2019); Shane Denson, Discorrelated Images (Durham: Duke University Press, 2020); Adam Hart, Monstrous Forms: Moving Image Horror across Media (Oxford: Oxford University Press, 2019).

9. Wolfgang Ernst, Chronopoetics: The Temporal Being and Operativity of Technological Media, trans. Anthony Enns (London: Rowman \& Littlefield, 2016), 205.

10. See Ernst, "Media Archaeology: Method and Machine," 239.

11. Alvin Toffler, Future Shock (New York: Random House, 1970).

12. See especially Pamela Lee, Chronophobia: On Time in the Art of the 1960 s (Cambridge, MA: MIT Press, 2004).

13. For more on wonder in animation, see Alan Cholodenko, "Introduction" to The Illusion of Life: Essays on Animation, ed. Alan Cholodenko (Sydney: Power Publications in association with the Australian Film Commission, 1991), 9-36; Tom Gunning, "Animating the Instant: The Secret Symmetry between Animation and Photography," in Animating Film Theory, ed. Karen Beckman (Durham, NC: Duke University Press, 2014), 37-53; Colin Williamson, Hidden in Plain Sight: An Archaeology of Magic and the Cinema (New Brunswick, NJ: Rutgers University Press, 2015).

14. Ernst, Chronopoetics, 4.

15. For more on the collapse of interest, funding, and research in AI from this time, see Daniel Crevier, AI: The Tumultuous Search for Artificial Intelligence (New York: Basic Books, 1993).

16. Volodymyr Mnih, Koray Kavukcuoglu, David Silver, Andrei A. Rusu, Joel Veness, Marc G. Bellemare, Alex Graves, Martin A. Riedmiller, Andreas Fidjeland, Georg Ostrovski, Stig Petersen, Charles Beattie, Amir Sadik, Ioannis Antonoglou, Helen King, Dharshan Kumaran, Daan Wierstra, Shane Legg and Demis Hassabis. "Human-Level Control through Deep Reinforcement Learning," Nature 518 (2015): Methods.

17. Terrence J. Sejnowski, The Deep Learning Revolution (Cambridge, MA: MIT Press, 2018), 171-172.

18. See D.H. Hubel and T.N. Wiesel, "Shape and Arrangement of Columns in Cat's Striate Cortex," The Journal of Physiology 165.3 (1963): 559-68; David H. Hubel, Eye, Brain, and Vision (New York: W.H. Freeman, 1988).

19. Hubel, Eye, Brain, and Vision, 131.

20. Yann LeCun, Léon Bottou, Yoshua Bengio, Patrick Haffner, "Gradient-Based Learning Applied to Document Recognition," Proceeding of the IEEE 86.11 (November 1998): 2283-2284. 
21. Mnih, et al., "Human-Level Control," 529.

22. See Frank Rosenblatt, "The Perceptron: A Perceiving and Recognizing Automaton," Report 85-60-1, Cornell Aeronautical Laboratory (Buffalo, New York: 1957); and Frank Rosenblatt, "The Perceptron: A Probabilistic Model for Information Storage and Organization in the Brain," Psychological Review 65 , no. 6 (1958): 386-408.

23. "New Navy Device Learns by Doing: Psychologist Shows Embryo of Computer Designed to Read and Grow Wiser," New York Times, 8 July 1958, 25.

24. Frank Rosenblatt, "The Perceptron: A Probabilistic Model for Information Storage and Organization in the Brain," quoted in Rudolf Seising, "The Emergence of Fuzzy Sets in the Decade of the Perceptron-Lotfi A. Zadeh's and Frank Rosenblatt's Research Work on Pattern Classification," Mathematics 6, no. 7 (2018): 110.

25. Sejnowki, 47 .

26. Mnih, et al., "Human-Level Control," 532.

27. Stephanie Boluk and Patrick LeMieux, Metagaming: Playing, Competing, Spectating, Cheating, Trading, Making, and Breaking Videogames (Minneapolis: University of Minnesota Press, 2017), 8.

28. Patrick LeMieux, "From NES-4021 to moSMB3.wmv: Speedrunning the Serial Interface," Eludamos: Journal for Computer Game Culture 8, no. 1 (2014): 12.

29. Mnih, et al., "Human-Level Control," 531.

30. Karin Knorr Cetina, "Objectual Practice" in The Practice Turn in Contemporary Theory, ed. Theodore R. Schatzki, Karin Knorr Cetina, and Eike von Savigny (London and New York: Routledge, 2001), 190.

31. Lisa Gitelman, Paper Knowledge: Toward a Media History of Documents (Durham: Duke University Press, 2014), 1.

32. Hodge, Sensations of History, 16.

33. For details about DeepMind's use in Starcraft II see Oriol Vinyals, Timo Ewalds, Sergey Bartunov, Petko Georgiev, Alexander Sasha Vezhnevets, Michelle Yeo, Alireza Makhzani, Heinrich Küttler, John Agapiou, Julian Schrittwieser, John Quan, Stephen Gaffney, Stig Petersen, Karen Simonyan, Tom Schaul, Hado van Hasselt, David Silver, Timothy P. Lillicrap, Kevin Calderone, Paul Keet, Anthony Brunasso, David Lawrence, Anders Ekermo, Jacob Repp and Rodney Tsing, "StarCraft II: A New Challenge for Reinforcement Learning," ArXiv abs/1708.04782 (2017): n. pag.; Vinicius Zambaldi, David Raposo, Adam Santoro, Victor Bapst, Yujia Li, Igor Babuschkin, Karl Tuyls, David Reichert, Timothy Lillicrap, Edward Lockhart, Murray Shanahan, Victoria Langston, Razvan Pascanu, Matthew Botvinick, Oriol Vinyals, Peter Battaglia, "Deep Reinforcement Learning with Relational Inductive Biases," International Conference on Learning Representations, 2019.

34. See, for example, Jonathan Sterne, The Audible Past: The Cultural Origins of Sound Reproduction (Durham: Duke University Press, 2003) and David Parisi, Archaeologies of Touch: Interfacing with Haptics from Electricity to Computing (Minneapolis: University of Minnesota Press, 2018). 
35. Bernard Dionysius Geoghegan, "An Ecology of Operations: Vigilance, Radar, and the Birth of the Computer Screen," Representations 147, no. 1 (Summer 2019): 59-95.

\section{About the Author}

Andrew R. Johnston is Associate Professor in the Department of English, the Film Studies Program, and the Communication, Rhetoric, and Digital Media Program at North Carolina State University. His book Pulses of Abstraction: Episodes from a History of Animation (University of Minnesota Press, 2020) examines abstract animation in cinema and computational media from the 1950s-70s. 


\title{
8. Intervals of Intervention: Micro- Decisions and the Temporal Autonomy of Self-Driving Cars
}

\author{
Florian Sprenger
}

\begin{abstract}
Self-driving vehicles do not simply translate algorithmic definitions of their interaction with the environment into material actions. In the implementation of microdecisions, temporality itself becomes an element of the success of operations. Taking the fascination for a non-human and distributed capability of decision-making as a starting point, the paper explores how the temporality of microdecisions is integrated into technical systems that interact with their surroundings. On the basis of a media archaeology of these temporalities, it develops a heuristic of autonomous technologies that explores the role of micro-decisions. With self-driving cars, terms such as agency (based on algorithms), temporality (in different intervals of intervention), decision (in reference to alternative scenarios), and autonomy achieve new meanings worthy of a re-interpretation.
\end{abstract}

Keywords: self-driving cars, autonomy, microdecisions, microtemporality

In September 2016, car manufacturer Tesla rolled out update 8.o for its autopilot operating system including a new radar processing algorithm. Manufactured by Bosch, the mid-range radar sensor (MRR) installed below the car is also used by other car companies, but Tesla was the first to integrate a new function: with algorithms developed by machine learning, the onboard computer system, Nvidia's DRIVE PX 2, enhanced its capacity to analyze data about the movement of two cars in front. ${ }^{1}$ Beginning with this update, the system utilizes the fact that the radar signal bounces between the vehicle driving in front and the underground of the road in order to

Volmar, A. and K. Stine (eds.), Media Infrastructures and the Politics of Digital Time: Essays on Hardwired Temporalities. Amsterdam: Amsterdam University Press, 2021 DOI 10.5117/9789463727426_CHo8 
detect the shape and movement of objects ahead of the car driving in front, even if they are invisible to the driver and the car's visual sensors.

This new technology reconfigures the autonomous car's intervals of intervention, that means the temporality between the registration of an event and the according reaction. Two months after the update, two cars crashed on a Dutch highway at high speed. Fortunately, no one was injured in this accident. A video recorded by a dashcam in an uninvolved Tesla Model $X$ and published on YouTube by the driver - a Tesla enthusiast - shows how the Tesla's driving assistants react to the accident of the car driving in front and the car that was two cars in front of the Tesla before it actually happens. ${ }^{2}$ The Tesla brakes autonomously before the driver even has the chance to recognize that something is about to happen, much less to intervene. In the video, a warning signal is emitted and the car starts to reduce speed, but at that moment nothing unusual is visible on the highway. Only a few seconds later, we recognize that the car, by means of the new processing algorithm, predicted the collision before it happened by calculating the speed and movement of a car invisible to the driver. If it hadn't reacted autonomously in the short interval available, it might have crashed too, because traffic was fast and the accident came out of nothing. This interval of intervention was only available to the car, but not to the driver. After the fact, we understand that the time in which a reaction was possible remained below the threshold of human attention and that the car anticipated a crash that became visible to the driver only in its consequences. The car's and the driver's intervals of intervention did not overlap. The vehicle had to take into account the probability of a future in which it would crash. In an extremely short interval — shorter than human reaction time-it had to decide autonomously between this future and a reaction that might prevent it.

This video shows computational agency in action and raises a certain uncanniness. Specifically, there are two sources of uncanniness: the car's autonomous reaction to an approaching collision which remains invisible, and the temporal interval between the (invisible) event demanding a reaction and its initiation. The consequence of invisibility is the impossibility of a human reaction, and the speed of the car's reaction demonstrates an impasse of human and non-human agency. Both amounts to an uncanniness that forces us to reflect the temporality of human capabilities. Perhaps the driver could have reacted if the collision had been visible. They might even have anticipated the accident. But it is likely that the interval between anticipation and motoric reaction would have been too long. The autonomous car brakes before the incident, it reacts to a potential event, because its 
so-called Advanced Driver Assistance Systems (ADAS) compare speed and direction of all three cars and calculate the probability of a collision.

The accident is preempted because in the available interval, the car decides autonomously between different scenarios depending upon their calculated probability and initiates a motoric reaction. This whole process is a microtemporal act of decision, and this paper sets out to explore the implications of this temporality. Self-driving vehicles do not simply translate algorithmic definitions of their interaction with the environment into material actions. In the implementation of what I suggest we call micro-decisions, temporality itself becomes an element of the success of operations. The term micro-decision should be used as a heuristic, as a language of description that makes certain aspects of these technologies visible which would remain invisible with a solely technical vocabulary.

By focusing on amateur videos of pre-empted accidents rather than on the many videos of smooth autopilot driving also available, this paper turns to examples in which the operational microtemporality of the car not only replaces the driver's activities. The pre-emption of crashes, as will become clear, is itself a modus operandi. The accident, as a temporal event in which computational agency unfolds, has become a learning situation for the vehicle. ${ }^{3}$ Machine learning thus also implies that the machine has to make mistakes in order to learn. Vehicles might become fully automated at one point, but at the present moment it is important to keep in mind that automation is gradual and extends mainly to computer-supported maneuverand routing-procedures. As recent accidents show, these technologies are not yet as reliable as the manufacturers want the public to believe. The most advanced cars available at the time of writing are rather sophisticated software-based driving assistants with sensor-based emergency brake and lane assistants on what the Society of Automotive Engineers has defined as level 2, than autonomous self-driving cars that no longer rely upon a driver. On this level, the automated system is authorized to take full control of the car in specific situations (usually on a highway) while the driver can take her or his hands off the wheel, but must be able to intervene at all times. While the self-driving car without a driver is usually taken as the horizon of fully automated autonomy (and currently available as a test-service by Waymo in geofenced areas of California), the decisive challenges in understanding these technologies amount to understanding their microtemporality. The technologies operating on this level are already available.

Taking the fascination for a non-human and distributed capability of decision-making as a starting point, this paper explores how the temporality of micro-decisions is integrated into technical systems that interact with 
their surroundings. With self-driving cars and other autonomous technologies from everyday applications of the internet of things to unmanned drones, we observe a transition of digital technologies from the realm of the virtual into the environment. The perspective presented here argues that spatial embeddedness and situatedness into surroundings are only possible because of a specific temporal operationability which is based on micro-decisions - put bluntly, automated vehicles couldn't effectively interact with their environment if they weren't able to decide in fragments of seconds. In the words of engineers: "Driving a vehicle implies taking decisions continuously based on the current awareness of the vehicles situation and its likely evolution."4

\section{The Speed of Decisions}

Micro-decisions are algorithmic processes of digital technologies characterized by quantity, speed, and automation. ${ }^{5}$ Their temporality is an effect of the relation between the sheer numbers of calculations and the velocity of automated processing. They surmount in surpassing human capabilities. Their number and speed can only be accomplished by computers: their quantity is their quality. They are not instantaneous, though, because the execution of every micro-decision takes time - the car never reacts in "real time." I suggest to use the term decision, as a choice between alternatives, to delineate processual events which depend upon the openness of alternatives and the contingency of input, in this case from the car's surroundings. This temporality is denoted by the micro: its intervals are too short for human attention. Consequently, a description of autonomous cars as agents of micro-decisions needs to account for the larger infrastructures that make micro-decisions possible as enactments of automated agency in a temporality accessible only to digital computers.

As elements of what Katherine Hayles calls infrastructural "cognitive assemblages, ${ }^{\prime 6}$ micro-decisions constitute a new mode of power that we are yet in the process of understanding. Micro-decisions decide upon possibilities that shape life in digital cultures: who can move to a specific location and who can't, who has access and who has no access, who is connected and who is disconnected, probably also who is a potential victim of an accident and who is not. Such decisions are not associated with individual decision-makers; rather, they are effective because they take place automatically-in unfathomable numbers and as quickly as possible — according to fixed sets of rules. They represent the smallest unit and the technical precondition 
of a politics of autonomous, adaptive technologies - and of our potential opposition to it. They question traditional concepts of agency, because the act of deciding is separated from individual deciders. Automation depends on the time-critical analysis of the environment, the calculation of the probabilities of different futures and according reactions, and finally on "temporal 'resolution' and speeds of decision."7 In this sense, the technological autonomy of self-driving cars is an effect of a microtemporality that bestows the capacity of environmental interaction on them. A heuristic of autonomous technologies that covers the role of micro-decisions should therefore be accompanied by a media archaeology of these temporalities.

To understand the consequences of autonomy based on micro-decisions, it is necessary to establish new a vocabulary. ${ }^{8}$ In the description of the accident presented so far, a series of concepts was invoked that this paper attempts to rearrange in the following four chapters:

\section{Computational Agency and Cognitive Assemblages}

In order to understand how micro-decisions foster computational agency, it is important to situate them within the complex interrelations of technical and environmental elements that constitute a self-driving car as what Katherine Hayles calls a "cognitive assemblage." Its modes of operation can be subdivided into three categories: first, regulation and control of relevant mechanical properties, e.g. steering, braking, signaling etc.; second, monitoring the environment (supported by a host of sensors, optical cameras, GPS, radar, sonar, laser and Lidar) and processing this information (identification, pattern recognition, categorization); and third access to road maps and updated traffic information collected by vehicles and to databases and upgrades maintained by manufacturers.

In their capability of solving cognitive tasks, autonomous cars, their drivers and their environments constitute a cognitive assemblage: "As a whole, a cognitive assemblage performs the functions identified with cognition in general: flexibly responding to new situations, incorporating this knowledge into adaptive strategies, and evolving through experience to create new strategies and kinds of responses. ${ }^{\text {"10 }}$ In the dynamic unfolding of human and non-human, material and biological actors into distributed agency, which Hayles describes with this term, "the spectrum of decision-makers"1 is widely expanded. Following Hayles, micro-decisions can be regarded as crucial elements of cognitive assemblages characterized by constant time-critical processes of synchronization between their components. To refer to this 
concept of distributed agency is relevant in this context, because micro-decisions do not simply replace a central cognitive unit, but can be applied on different levels with varying tasks. Their agency is distributed within different layers of operationability, it is situated, embodied and interactive.

Autonomy does not mean that the car is independent of the other components of the assemblage, but a component of an infrastructure that endows specific actors with the capacity of decision-making and environmental agency. The media theoretical challenge lies in understanding this temporality not as the agency of a calculating device, but as an infrastructural effect of a "cognitive assemblage." In this regard, recent technological developments challenge traditional concepts of agency, autonomy, and decision. An investigation of these concepts has to constantly re-adjust the language of description. This approach may have another, unintended, but not unwanted consequence: what a human decision is and what constitutes the agency behind it may become more and more unclear.

\section{The Temporality of Reaction Time}

Temporality, so far, has referred to the intervals of intervention. But how are microtemporal decisions included into intervals of intervention? In the development of ADAS systems, the perception-response-time of human drivers and computers has become a "critical design element for vehicular safety systems, which interact with the driver."12 In this context, a look at the history of the concept of reaction time in experimental physiology reveals that this temporality is conceived of as neither a unitary process nor a simple reflex. Historical research on reaction time conceptualizes acts of decision as variables of intervals of reactions between an input (signal subject to decisions) and an output (pressing of a key). In the 1950s, this process was formulated in terms of information theory. In the concept of reaction time developed by cognitive sciences and employed by automotive engineers, the interval of reaction turns out as the time it takes for the experimental subject to decide between choosing and pressing a key or not pressing a key. On the basis of this research, decisions, as temporal variables of information processing, are integrated into the design of interfaces and control elements for the interaction between vehicles and human drivers. ${ }^{13}$ Accordingly, the automation of decisions as micro-decisions can be seen as an attempt to implement automated reaction times.

Contemporary conceptions of reaction time are strongly influenced by a seminal paper that experimental psychologist William Edmund Hick 
published in $1952 .{ }^{14}$ His approach to cognitive research draws upon nineteenth century experiments and reframes them through information theory. Influenced by Claude Shannon's work, this approach defines reaction time as the interval between input and output of stimulus and impulse, as the sum of "cognition time" and "choice time." Between cognition and choice, a decision, as a choice between alternatives, takes place about which reaction to a pre-defined stimulus is necessary.

Hick is interested in the rate of gain of information and, accordingly, the "mathematical relation between reaction time and number of alternatives."15 His experimental research determines the interval of a reaction depending on the number of alternative choices. In the experiments conducted by Hick, the decision consists in pressing one of up to ten keys if one of up to ten corresponding lamps flashes. The experiment ultimately shows that if more choices, that means a series of lamps, are added, or the frequency of flashing is raised, choice time increases logarithmically in analogy to Shannon's definition of information as a logarithmic function of alternatives. ${ }^{16}$ As a result, the duration of a decision appears as an effect of the number of alternative choices. Consequently, the interval of a decision is bound to the number of choice alternatives: the more alternatives, the greater the amount of information to be processed. With more alternatives, the level of uncertainty increases, which leads to a delay in reaction time. Autonomous technologies, as information-processing machines, correspondingly operate with probabilities of events that reduce the number of choices.

Hick's law, as it was subsequently called, only applies to situations in which decisions are simple and reactions correspondingly quick. Because it introduces a conception of reaction as information processing, this approach has become highly influential in cognitive sciences and in engineering all kinds of interfaces in which several options are presented. Measuring human reaction times on the basis of Hick's Law had a huge influence on the design of safety systems, road intersections and the car's interfaces. ${ }^{17}$ As for example a recent project by BMW on a decision-making algorithm for emergency brake assistants demonstrates,${ }^{18}$ Hick's law facilitates the correlation of human and non-human reaction times and demonstrates that the temporality of automation can be understood as the time of decisions - be they based on human or algorithmic agency. The act of decision takes place between input and output and as such it can be operationalized as micro-decisions.

The time it takes a self-driving car to react is influenced by different factors: the speed of calculation, depending upon the number of alternative choices, which is determined by the clock rate of the car's GPU (in the 
most advanced version, Nvidia's DRIVE PX 2 has a clock rate of 8 Teraflops (Floating Point Operations Per Second), which makes this factor almost negligible for reactions); the transmission times between the different modules of the car; and the frequency with which it scans its surroundings. The mid-range radar sensor mentioned in the beginning emits signals every 50 milliseconds (twenty times per second). The radar signal needs time to be transmitted depending upon the distance of the scanned objects. If the system decides to come to a stop, the Tesla iBooster brake system can reach full braking in 150 milliseconds. The time it takes to stop depends upon the vehicle's speed and the road conditions. Adding these times to the time needed for information processing results in an estimated reaction time. In this constellation of different temporalities, the decision-making process is the synchronizing element between the environment and the reaction of the car. In the sequence of events, it is the orchestrating factor.

\section{The Decidability of Alternative Futures}

The biggest challenge for autonomous vehicles is not only to constantly scan their environment and to map their surroundings, but to project the results of different possible reactions and to prevent accidents before they happen. Reacting in the time before an accident in a pre-emptive mode of environmental embeddedness means to realize an alternative future before the irrevocability of the accident precludes any alternatives. If an accident is to be pre-empted, this already implies a future in which the accident happens. Pre-emption operationalizes decisions, which rest upon the alternativity of its options. The main operation of the car's CPU is to constantly transform different futures into probabilities that can be calculated and require vast numbers of micro-decisions in extremely short time intervals. This differentiality of potential futures is the basis of the decidability.

To calculate the probabilities of different scenarios and possible reactions to them is not only a question of reaction, but also of prediction. "Critical traffic situations can require a decision among several unfavorable alternatives for action. Here again, virtual assessment can support the development of transparent decision algorithms." ${ }^{19}$ The car has to predict the probabilities of possible futures and choose a reaction according to a set of codes of behavior that can be either preprogrammed or be extracted from the diversity of enacted situations by machine learning. These rules can then be applied to new situations depending upon the pre-given goals 
defined beforehand in what can be called macrodecisions - for example that the safety of pedestrians is more valuable than the safety of passengers. Pre-programmed, determined reactions never comprise the contingency of the environment. The rules that the car (respectively the fleet) has learned and that were coded into its algorithmic set-up are not a set of reactions in which ethical assumptions are simply translated into algorithms, but conditions of probabilities, as Lucy Suchman and Jutta Weber argue: "The behavior is not pre-programmed, but rather the outcome of a kind of systematized tinkering and situated experimenting of the system with its environment." ${ }^{\text {20 }}$ Recognized patterns can be used as predictors for future behavior, as Jack Stilgoe stresses in his analysis of machine learning for self-driving cars: "Deep learning systems are seen by their creators as means of engaging with an uncertain world that is impossible to capture with a set of formal rules. However, in developing rules, such systems may create new social uncertainties. In gaining the ability to recognize and make decisions about unfamiliar information, they lose the ability to account for their actions." ${ }^{21}$ In other words: the car has to make decisions under conditions of uncertainty and at the same time creates further uncertainty as an actor in the environment.

In the example discussed so far, prediction and action conjoin to arrive at a decision without a human decider and their agency. The resulting cognitive assemblage of automated traffic, I want to argue, cannot be explained by the operationability of a algorithms. Rather, it is important to call them decisions with all the conceptual consequences this entails, instead of trivializing them as predetermined executions of fixed rules. The act of deciding, traditionally embedded in concepts of intentionality, but now fulfilled by machines, has to be rethought in its microtemporality. ${ }^{22}$

The heuristics of calling these processes decisions follows the intuition that as decisive acts they take place in the temporal interval of interruptions. Interruptions, in the tact of digital processing, are the precondition for decisions, because they interrupt in order to introduce an alternative. ${ }^{23}$ Micro-decisions are more than the processing of a determined sequence of calculations - they always decide between possibilities in an interruption. Decisions require time. Reactions require decisions. Interruptions free up this time by adding durations of stasis to the temporality of transmissions. The duration of calculation, not its moment is decisive.

To use the term decision takes into account their openness. In this sense, a decision is never absolute-it always implies an alternative. A decision can always be different. As Niklas Luhmann put it in a paper on decisions in formal organizations: "It [the decision] constructs the alternativity of 
its alternative regarding "what could be," and it always constructs it in the present." ${ }^{24}$ Luhmann points out that decisions depend upon the potentiality of alternatives and consequently also upon the difference between past and future. For the observer, "the decision before the decision is different from the decision after the decision." ${ }^{25}$ While the openness of the decision before the decisive act lies in the multiplicity of alternatives, that means the contingency of the future, a decision after the decisive act appears as what Luhmann calls "thickened contingency": ${ }^{26}$ it could have been different, but it is fixed.

To speak of decisions instead of programs, rules, or algorithms opts to unclose this potential of contingency. The basic fact of decidability-in opposition to determination - is important here. Acts of decision-and this is central for the scope of my argument - are decisions not only between two or more alternative chains of reaction, but also between different futures. In a technical and mathematical context, a de-cision (Ent-Scheidung) is more than the execution of a predetermined protocol or a programmed algorithm, more than the definition of a possible answer. A decision is always an act that draws a distinction and requires an alternative. As predictions of possible outcomes, they are bound to the future. In order to decide, a self-driving car needs alternatives that are in turn products of algorithmic prediction and the calculation of probabilities.

Alternativity is also the reason why micro-decisions should not be mistaken for algorithms. Micro-decisions and algorithms are situated on different conceptual levels. Micro-decisions are coded as algorithms, but they only become operational in a quantity, speed and automation which cannot be reduced to algorithms. Not all executions of algorithms are micro-decisions - algorithms can be slow, analog and based on human labor.

Nonetheless, it is obvious that the importance of algorithms has escalated since the rise of digital computing in the second half of the twentieth century due to new velocities of calculation. But even though the speed of execution might be important for the application of specific algorithms, this temporality is not a necessary condition for their implementation. In this sense, the term algorithm does not encompass temporality, which is the key element of automated decision-making. ${ }^{27}$ Algorithms are temporally nonspecific and can in principle be performed by a human or a machine at any speed. While algorithms can be translated back into human work, the speed and quantity of micro-decisions are not substitutable. The focus on algorithms prevalent in recent media theoretical discussions conceals that even though micro-decisions are programmed as executions of algorithms, their effects lie in their temporality and automation which should not be mistaken as effects of algorithms. 


\section{The Time of Autonomy}

The dimension of autonomy presented so far challenges traditional conceptions of agency and accountability. In this regard, the automation of traffic leads to a reversal of contingency of the so-called trolley problem, ${ }^{28}$ which results in juridical and ethical grey areas. Though the ethical dimension of automated, time-critical technologies cannot be discussed here extensively, the following remarks on the trolley dilemma serve to demonstrate how autonomy and microtemporality are intertwined.

The trolley dilemma was introduced by Philippa Foot in her seminal paper The Problem of Abortion and the Doctrine of the Double Effect in 1967. This scenario touches the roots of ethical considerations by negotiating human interaction with technology. Foot describes a runaway tram driver who has to decide between two tracks: five people are working on track one and one person on track two. Anyone of them is bound to be killed if the tram takes the track. With the rise of automated cars, the resulting ethical dilemma needs to be reformulated: How should the car react in a situation in which the driver cannot intervene? For example, a car may have to decide if it rather takes the risk of destroying itself and killing the driver or of crashing into a group of people certainly killing several of them. ${ }^{29}$ The new challenge lies in the fact that decisions about life and death and the accompanying responsibilities might be outsourced to algorithms. In such a scenario, responsibility couldn't be accredited to a human actor-a problem even more urgent since the first deadly crash with an automated car in May 2016, in which a Tesla wrongly identified a blue truck for the sky. ${ }^{30}$

This discussion about agency and accountability has become very popular, as its steady recurrence in newspapers and publications shows. So far, these discussions have not registered the importance of temporality for the trolley driver's decision. Usually, descriptions of the dilemma do not account for the time, respectively for the urgency of the decision that needs to be made. In the scenario described by Foot, it is not mentioned that the driver of a tram only has a few seconds to decide between their options of killing five human beings or one. The dilemma, I want to argue, consists not only in the fact that both decisions result in casualties, but is multiplied by the fact the driver doesn't have the time to reflect their options or even the philosophical implications of their decision. The interval of intervention, which is not covered by Foot's argument, needs to be taken into account in contemporary ethical discussions, because the microtemporality of decisions - not accessible for the human driver-is operationalized in 
automation. While a human driver might be unable to come to a rational conclusion in a few seconds, the car's micro-decisions are perfectly rational - though not necessarily convincing — because they are calculated in the speed of data processing.

In how far the ethical discussions have to be transformed by taking into account microtemporalities is a question that has to be left to experts in this field. In the context of this paper, it is important to note that automation and decision-making are connected on the basis of microtemporality. In the words of engineers again: "This challenge leads to relevant implications on the design of the automated driving system: the automated system has to make decisions in all situations and cannot rely on the driver to take back responsibility instantly."31 As this quotation shows, engineers deduce the autonomy of a vehicle from its potential to decide in all situations, which means at all times and in all temporalities. Micro-decisions thus can be seen as a core element of autonomy.

At the current state of technology, though, the autonomy of decisions is a messy business. Practically, the experience of driving a Tesla shows the need to balance agencies of decision and to constantly revise them. In a study on Tesla's Model $S$, Mica Endsley has demonstrated that the different systems of automation embedded in the car result in a series of complex situations of decision-making on behalf of the driver. Agency is constantly re-distributed. The technologies employed by a Model $S$ at the time of Endsleys research in late 2016 (version 7.2) included Adaptive Cruise Control (ACC) to adjust its speed to traffic, Autosteer to hold the line and Auto Lane Change. Anxieties arise for example because the ACC reacts if the car in front brakes or stops, but not at traffic lights. Auto Lane Change seems unreliable in detecting cars next to the Tesla. Endsley argues that while driving on a highway is indeed automated to a large extent, all other traffic situations need constant intervention and attention by the driver. ${ }^{32}$ In this regard, the automated execution of specific routines also creates the possibility of distraction. In situations in which the machine is unable to decide and the human driver has to intervene, distinct and intuitive interfaces are required that address the driver's situational awareness in an unmistakable way. The comfort of automated driving, Endsley argues, may lead to a slowdown of attention and, consequently, of reaction time. The most important task for designers is the integration of different modes of attention through human-computer-interaction and mental models of the car. This, in fact, turns out to be an integration of different levels of decision-making - and an integration of different reaction times as intervals of decisions. ${ }^{33}$ 
In this respect, the concept of micro-decisions may prove to be relevant for a discussion of the agency in question and finally for new concepts of the interaction between drivers and cars. Autonomous technologies can be understood as situated technologies capable of adaptation to their surroundings. As Suchman and Weber have demonstrated in the context of unmanned drones, "the project of machine intelligence is built upon, and reiterates, traditional notions of agency as an inherent attribute and autonomy as a property of individual actors." ${ }^{34} \mathrm{As}$ an alternative in the vein of science and technology studies, they propose a conception of agency as relational, embedded in human-machine-assemblages and constantly re-configured. In addition to this perspective, the approach presented here supposes to take the temporality of micro-decisions into account for the constitution of computational agency and autonomy.

\section{Conclusion}

The uncanny that may creep upon us while watching the video with which this paper started is a symptom for the necessity to rethink our relation to such technologies. To speak about decisions as their defining characteristic and to borrow this concept from social theory does not yet entail that there is any social intention behind their operations. Machines do not (yet?) have intentionality in the sense of conscious acts or reflexivity. But they can, as philosopher Peter-Paul Verbeek suggests, achieve intentionality in the sense of intendere: they can realign and orient something. ${ }^{35}$ The alternative between and mutual exclusion of intentional, reflexive human decision making and deterministic, mechanical procedures is misleading. Machines that carry out micro-decisions are of course produced and managed by human agents, who also program their protocols and algorithms. The measures according to which decisions are made are necessarily established in protracted institutional negotiations between various interest groups, manufacturers, regulators, engineers and coders, obvious in ethical discussions about the implementation of life-threatening reactions in autonomous vehicles and the juridical problems this entails. The implementation of micro-decisions is based on collective or individual macrodecisions. Yet, the great mass of micro-decisions can only be executed by computers, and it is this mass that underlies the technical definition of successful digital communication or traffic on computer-supported networks. Micro-decisions have become as effective as they are precisely because they circumvent the laborious human act of decision-making on the basis of calculated probabilities. 
Assuming the determinacy of micro-decisions by machines results in a simplistic division between determinate computational agency and free human agency. What is determined cannot be decided. In the words of Niklas Luhmann, decisions imply a "minimum of unpredictability." ${ }^{6}$ While it is obvious that human agency and human decision-making are different from computational agency, that does not mean that we should imagine agency only as a blueprint of human agency against which machinic agency would seem to be deterministic. The concept of micro-decisions wants to circumvent the shortcomings of decisional determinism by taking into account three aspects. First, conceptualizing micro-decisions as predetermined acts of rendering a preprogrammed set of rules would also mean that such decisions cannot be changed and are bound to past events instead of anticipating and regulating the future. Second, if we define micro-decisions as determined, we would not be able to understand the technological developments that allow for example an autonomous car to react to the complexity and contingency of its environment. Calculative environmental technologies need to account for the unpredictable. ${ }^{37}$ Third, defining decisions as deterministic would imply that they are immediatethat they happen without taking time. The fact that micro-decisions - and that no transmission and no medium - can ever be immediate, that they always take time, leads to a non-deterministic, decisive understanding of micro-decisions. This means that the politics of micro-decisions, including potentials to transform them, are based upon the interruption of connection and the fact of constant disconnection.

To speak about decisions also means to bear in mind that no decision is ineluctable and that every decision can be reached in a different mannerthat it is possible to modify them for the better, but that they can also turn out for the worse. Yet even a bad decision is better than no decision, which leaves no room for improvement-for example on how autonomous cars react to specific situations in which people are in danger. To make decisions in advance, to determine them, or even to abolish the act of decision-making, is, in every case, to reduce what is possible. Nonetheless, micro-decisions are neither inherently good nor inherently bad. For the operations of a network such as that of automated traffic, however, they are unavoidable. This means that we need a conceptual framework that helps us to understand their mode of power in its microtemporal dimension. To simply delegate decisions to machines and then to conceive them as determined algorithms, as something that necessarily happens as it happens, is at core a depoliticizing act. In this regard, it is the task of critical humanities to politicize machines. This means to take the alternatives of each decision, be it fast or slow, as a starting point. 


\section{Notes}

1. Tesla (blog), Tesla, September 26, 2016, https://www.tesla.com/de_DE/blog/ upgrading-autopilot-seeing-world-radar. For a demonstration, see Eric Loveday, "Tesla Model X Sees Two Vehicles Ahead In Automatic Braking TestVideo," Inside EVs, November 10, 2016, https://insideevs.com/tesla-model$\mathrm{x}$-sees-two-vehicles-ahead-automatic-braking-test-video/. Autopilot here means the combination of different Advanced Driving Assistance Systems: collision warning, autosteer lane centering, self-parking, automatic lane changes, and traffic-aware cruise control. In this regard, Tesla offers a good example of the constraints, challenges, and potentials of driving assistants, although it is certainly not the only competitor in the field.

2. Fred Lambert, "Tesla Autopilot's New Radar Technology Predicts an Accident Caught on Dashcam a Second Later," Electrek, December 27, 2016, https://electrek.co/2016/12/27/tesla-autopilot-radar-technology-predict-accident-dashcam/.

3. See Paul M. Leonardi, Car Crashes without Cars: Lessons about Simulation Technology and Organizational Change from Automotive Design (Cambridge, MA: MIT Press, 2012).

4. Javier Ibanez-Guzman, Christian Laugier, John-David Yoder, and Sebastian Thrun, "Autonomous Driving: Context and State-of-the-Art," in Handbook of Intelligent Vehicles, ed. Azim Eskandarian (Berlin: Springer, 2012), 1286.

5. See Florian Sprenger, Politics of Microdecisions: Edward Snowden, Net Neutrality and the Architecture of the Internet (Lüneburg: Meson Press, 2015).

6. See N. Katherine Hayles, Unthought: The Power of the Cognitive Nonconscious (Chicago: University of Chicago Press, 2017).

7. Axel Volmar, "Zeitkritische Medien im Kontext von Wahrnehmung, Kommunikation und Ästhetik," in Zeitkritische Medien, ed. Axel Volmar (Berlin: Kadmos, 2009), 9-26, 10.

8. In a similar direction, Beatrice Fazi has argued that "computational processes of decision-making and prediction might constitute a novel modality of thought: not despite the deterministic, deductive, axiomatic, procedural and operational character of these processes, but rather because of it." M. B. Fazi, "Can a Machine Think (Anything New)? Automation Beyond Simulation," AI \& Society 34, no. 4 (2018), 10. In addition to Fazi's argument, the emphasis here rests on the temporality of these processes.

9. See Hayles, Unthought.

10. Hayles, Unthought, 118-19. Hayles prefers the term assemblage over network, because it denotes "continuity in a fleshy sense," while network implies "a sense of sparse, clean materiality" (118).

11. Hayles, Unthought, 115 .

12. Azim Eskandarian, "Fundamentals of Driver Assistance," in Handbook of Intelligent Vehicles, ed. Azim Eskandarian (Berlin: Springer, 2012), 491-535, 499. 
13. See Katherine L. Plant and Neville A. Stanton, "Identifying the Importance of Perceptual Cycle Concepts during Critical Decision making in the Cockpit," Procedia Manufacturing 3 (2015).

14. For the importance of Hick, see Robert W. Proctor and Darryl W. Schneider, "Hick's Law for Choice Reaction Time: A Review," Quarterly Journal of Experimental Psychology 106, no. 16 (2018). On the history of physiological research on reaction times see Henning Schmidgen, "The Donders Machine: Matter, Signs, and Time in a Physiological Experiment, c. 1865," Configurations 13 (2005); and Jimena Canales, A Tenth of a Second: A History, Pbk. ed. (Chicago: University of Chicago Press, 2011).

15. W. E. Hick, "On the Rate of Gain of Information," Quarterly Journal of Experimental Psychology 4, no. 1 (1952): 11. Hick's law is the basis for a model of intelligence in which the speed of information processing is an indicator of intelligence. Though he doesn't use the term decision and talks about choice, the term has become common in the application of Hick's law.

16. In a follow-up article published a year later, Ray Hyman underlines that "the choice-reaction-time experiment can be looked upon as a model of a communication system" (Ray Hyman, "Stimulus Information as a Determinant of Reaction Time," Quarterly Journal of Experimental Psychology 45 , no. 3 (1953): 188) and determines the bits of information per stimulus presentation.

17. See Tibor Petzoldt and Josef F. Krems, "How Does a Lower Predictability of Lane Changes Affect Performance in the Lane Change Task?," Applied Ergonomics 45, no. 4 (2014). and Chia-Fen Chi and Ratna S. Dewi, "Matching Performance of Vehicle Icons in Graphical and Textual Formats," Applied Ergonomics 45, no. 4 (2014).

18. See Philipp Reinisch, Peter Zahn, and Dieter Schramm, "Using a Reaction Time Model for Determining a Collision Avoidance System's Brake Timing," IFAC Proceedings Volumes 43, no. 7 (2010). For other examples, see Marc Green, “'How Long Does It Take to Stop?': Methodological Analysis of Driver Perception-Brake Times," Transportation Human Factors 2, no. 3 (2000).

19. Thomas Helmer et al., "Safety Performance Assessment of Assisted and Automated Driving in Traffic Simulation as Knowledge Synthesis," in Automated Driving, ed. Daniel Watzenig and Martin Horn (Berlin: Springer, 2017), 473-94, 492.

20. Jutta Weber and Lucy Suchman, "Human-Machine Autonomies," in Autonomous Weapons Systems: Law, Ethics, Policy, ed. Nehal Bhuta et al. (Cambridge: Cambridge University Press, 2016), 75-102, 88.

21. Jack Stilgoe, "Machine Learning, Social Learning and the Governance of Self-Driving Cars," Social Studies of Science 48, no. 1 (2017): p. 6.

22. The concept of decision has been discussed in many contexts, but so far not systematically applied to technologies. See for example Derek P. McCormack and Tim Schwanen, "Guest Editorial: The Space-Times of Decision Making," Environment and Planning A 43, no. 12 (2011); Peter Adey and Ben Anderson, "Event and Anticipation: UK Civil Contingencies and the 
Space-Times of Decision," Environment and Planning A 43, no. 12 (2011), doi:10.1068/a43576.; Florian Hoof, "Medien managerialer Entscheidung: Decision-Making 'At a Glance,” Soziale Systeme 20, no. 1 (2016).

23. On the importance of interruptions for micro-decisions see Sprenger, Politics of Microdecisions, p. 86.

24. "Sie konstruiert die Alternativität ihrer Alternative unter dem Gesichtspunkt 'was sein könnte;' und sie konstruiert sie in ihrer Gegenwart." Niklas Luhmann, "Die Paradoxie des Entscheidens," Verwaltungs-Archiv 84, no. 3 (1993): 291. All translations my own.

25. "Die Entscheidung vor der Entscheidung [ist] eine andere Entscheidung als nach der Entscheidung." Luhmann, "Die Paradoxie des Entscheidens," 293.

26. In Luhmann's original German, "verdichtete Kontingenz." Luhmann, "Die Paradoxie des Entscheidens," 293.

27. That does not imply that algorithms are unimportant for automated decisions, see Serjoscha Wiemer, "Von der Matrix zum Milieu: Zur Transformation des Entscheidungsbegriffs zwischen homo oeconomicus und evolutionärer Auslese," in Medien der Entscheidung, ed. Tobias Conradi, Florian Hoof and Rolf F. Nohr (Münster: Lit, 2016), 23-46.

28. See Philippa Foot, "The Problem of Abortion and the Doctrine of the Double Effect," Oxford Review, no. 5 (1967).

29. See Jean-Francois S. A. Bonnefon and Iyad Rahwan, "The Social Dilemma of Autonomous Vehicles," Science 352, no. 6293 (2016), doi:10.1126/science. aaf2654. For the difference between accountability and responsibility see Maya I. Ganesh, "Entanglement: Machine Learning and Human Ethics in Driver-Less Car," Aprja 6, no. 1 (2017). For a critique of the applicability of the trolley dilemma, see Tobias Matzner, "Autonome Trolleys und andere Probleme: Konfigurationen Künstlicher Intelligenz in ethischen Debatten über selbstfahrende Kraftfahrzeuge," Zeitschrift für Medienwissenschaft 21 (2019), 46-55, doi:10.25969/mediarep/12632

30. US Department of Transportation, National Highway Traffic Safety Administration, ODI Resume, June 28, 2016, https://static.nhtsa.gov/odi/inv/2016/ INCLA-PE16007-7876.pdf. See Victoria A. Banks, Katherine L. Plant, and Neville A. Stanton, "Driver Error or Designer Error: Using the Perceptual Cycle Model to Explore the Circumstances Surrounding the Fatal Tesla Crash on 7 th May 2016," Safety Science 108 (2018), doi:10.1016/j.ssci.2017.12.023.

31. Jan Becker, Maria-Belen Aranda Colas, and Nordbruch Stefan, "Bosch's Vision and Roadmap Toward Fully Autonomous Driving," in Road vehicle automation, ed. Gereon Meyer and Sven Beiker (Heidelberg: Springer, 2014), 49-6o, p. 5 o.

32. From an engineering point-of-view see also Victoria A. Banks and Neville A. Stanton, "Keep the Driver in Control: Automating Automobiles of the Future," Applied Ergonomics 53 (2016), doi:10.1016/j.apergo.2015.06.020.

33. Some engineers even argue that human agency needs to be included for automation to become effective: Neville A. Stanton and Philip Marsden, "From Fly-by-wire to Drive-by-wire: Safety Implications of Automation in 
Vehicles," Safety Science 24, no. 1 (1996). See also Sam Hind, "Digital navigation and the driving-machine:: Supervision, calculation, optimization, and recognition," Mobilities 20, no. 1 (2019).

34. Jutta Weber and Lucy Suchman, "Human-Machine Autonomies," in Autonomous Weapons Systems: Law, Ethics, Policy, ed. Nehal Bhuta et al. (Cambridge: Cambridge University Press, 2016), 75-102, 98.

35. Peter-Paul Verbeek, "Ambient Intelligence and Persuasive Technology: The Blurring Boundaries Between Human and Technology," NanoEthics 3, no. 3 (2009).

36. Luhmann, "Die Paradoxie des Entscheidens," 287. In Luhmann's original German, "ein Mindestmaß an Unvorhersagbarkeit."

37. While Luciana Parisi describes algorithmic decisions with Alfred North Whitehead's process ontology as modi of prehension, the focus here rests on their technical implementation: "The computation of relations thus requires that preplanned decisions become replaced by prehensive capacities of decision making, which afford the parametric system the freedom to establish unintended connections between parameters within the constrained conditions of sequential programming." Luciana Parisi, Contagious Architecture: Computation, Aesthetics, and Space (Cambridge: MIT Press, 2013), 138.

\section{About the Author}

Florian Sprenger is Professor for Virtual Humanities at the Institute for Media Studies at the Ruhr-Universität Bochum. His research topics are environmental technologies, transformations of traffic, and autonomous technologies. 


\section{Part III}

Lifetimes 



\title{
9. Grounded Speed and the Soft Temporality of Network Infrastructure
}

\author{
Nicole Starosielski
}

\begin{abstract}
:
This chapter offers a descriptive view of cable temporality from the perspective of network operators and those involved in system maintenance and repair. Examining the "soft temporalities" of these operators' labor, the chapter illustrates a tension at the heart of media's infrastructures: in many places, slowness, stability, embeddedness, and fixity of infrastructures are what enable speed and acceleration. After describing the "grounded speed" of the cable network, the chapter turns to the phenomemon of temporal irruptions: moments when the assemblage of temporal processes that enable network operation and network speed suddenly and radically changes the network.
\end{abstract}

Keywords: undersea cable, infrastructure, slowness, telecommunications, network operation

Outside the thick concrete walls of a Pacific Island undersea cable station, the dense heat draws tourists to the beach and into the ocean. Many have come here to break from the accelerated rhythms of hyper-stimulated lives-a separation that some see as moving "out of time."1 For others, "island time" is a staged interruption in their acceleration, one that incorporates them into longstanding colonial temporal regimes. Inside the station, a different temporality is in operation. It is evident in the chilled air, where molecules have been slowed to an optimal temperature for machine operation. And it is evident in the building's sparse population, which exists in stark contrast to the crowded beaches. On the day that I visit the station, there are only a few employees on the clock. As one of them gives me a tour, he points to a laptop on top of a stack of servers. Here, he tells me, he could play

Volmar, A. and K. Stine (eds.), Media Infrastructures and the Politics of Digital Time: Essays on Hardwired Temporalities. Amsterdam: Amsterdam University Press, 2021 DOI 10.5117/9789463727426_CHо9 
video games with the least possible latency. His computer's position in the network's geography gives him a slight temporal advantage in real-time multiplayer online games. He is describing the spot-in a concrete building intensely insulated from the rest of the island, an island that is visited for its remoteness - where he is as close as possible to achieving network instantaneity. It is the place where his signals would travel faster than anyone else's, even in the many cities the nearby visitors have come to escape. Metaphorically and thermodynamically, this is both the hottest and the coldest point on the island. The temporality of the cable station inverts the rhythms of bodies beyond the walls: here, it is not that slowness provides a break from acceleration, but rather, that slowness makes possible extraordinary speed.

A century earlier, on another island in this same ocean, telegraph operators shared a similar sense of spatial and temporal disjuncture as they occupied the center and periphery at once. Sharing their stories in the cable industry magazine, they reflected on "the monotony of our slumberous existence," challenged one another to prove that they lived in the loneliest, most remote station, and looked forward even to the transient missionaries that might drop by in the summer and provide the smallest diversion from the banality of everyday life. ${ }^{2}$ The slowness of their world existed in stark contrast to the intensity, speed, and pace of the network they operated. This was often commented upon in stories and the company's internal communications. One operator remarked: "Though they have to wait three months for letters to be forwarded from Honolulu ... men on Midway can obtain news from nearly all parts of the world in a few minutes." ${ }^{3}$ For these telecommunications workers, as for the people they connected, the acceleration of communications could heighten the relative sense of the slowness of their nonelectric surroundings, including their own bodies and the labor that they performed.

These two examples, one from the early twentieth century and the other from a hundred years later, illustrate a critical point about the temporality of networked media: the speed of communication is often made possible by incredible investments in stasis, stability, and slowness-molecular, bodily, and architectural. While much interest in infrastructure's temporalities and microtemporalities has focused on acceleration, less research has examined the temporal dilations and prolongations that make acceleration possible. This, as Sarah Sharma points out in her foundational text In the Meantime, is a critical blind spot of speed theory. ${ }^{4}$ As she identifies in works from Paul Virilio's Speed and Politics to Jonathan Crary's 24/7: Late Capitalism and the Ends of Sleep, the focus on speed, time-space compression, and acceleration 
is often conducted from a position of temporal privilege, and often fails to account for the multiplicity of lived times that scaffold acceleration. ${ }^{5}$ This chapter brings Sharma's argument to bear on media infrastructures. It offers a descriptive view of cable temporality from the perspective of network operators and those involved in system maintenance and repair. Following Gabriele Schabacher in this volume, it sees hardwiredness "as a network effect with relative duration brought about by specific types of labor." If we consider the operations of these systems as solely a technical process, rather than a performance of situated and geopolitically-specific labor of care, we miss the complex, embodied temporalities of contemporary digital infrastructure. And as a result, we fail to account for the decisions involved in establishing, maintaining, and securing network infrastructure.

Like the taxi-cab drivers that transport global jetsetters in Sharma's study, there is a population whose everyday work and temporal coordination makes possible the speed of the network-in the case of the cable system, these include network operators, engineers, and suppliers, among many others. The everyday work of cabling involves, for today's operators, waking up in the middle of the night, interrupted from sleep by a broken cable. It includes the coordination of ship movements with weather patterns and the active routing of internet traffic. One thing that distinguishes network operators from many other laborers that maintain the acceleration of the global elite is a co-presence of extraordinary temporal privilege (and a concentrated personal investment in that speed) alongside a sense of an extraordinary slowness, prolonged activities of care, and relative stability. While this in itself is not unique-living in the midst of contradictory temporalities and oppositional rhythms is characteristic of modern life- the temporalities of these operators' labor illustrate a tension at the heart of media's infrastructures: in many places, slowness, stability, embeddedness, and fixity of infrastructures are what enable speed and acceleration. From the operator's standpoint, especially those that inhabit the cable station, these two extremes are often brought together in poetic opposition, innately intertwined, and perceptible in their bodies as an affect of remote centrality. I call this dynamic grounded speed: the ongoing production of accelerated rhythms through the consistency and regularity of network bodies, architectures, practices, and environments.

In the first half of this chapter, I describe the grounded speed of the undersea cable system. As a background for this analysis, I begin with a narrative about the larger-scale temporal patternings of the cable network. Some of the common descriptions of cable temporalities include: cables erase time; cables accelerate; and network infrastructures are significant 
because of their high speed and their microtemporal operations. In contrast to these typical observations, the second section reveals some of the varied decelerations, slowings, and stabilizings that make possible cable speed. First and foremost is the slowness of human navigation through the ocean. The slowness and sparseness of marine transport has produced relative safety for cable systems: more boats would produce more cable breaks. Next to this is the historical privileging of remote areas for centers of network traffic, where the frequency of human and machinic movement is less likely to disrupt or interfere with cable traffic. Lastly is the relative slowness of the cable industry, an insular community where extended relationships between operators emerge over years. This social world reproduces the cable network as an intimate and familiar landscape. Tracking cable industry labor, past and present, I show how the slowness of the network's milieu grounds the speed of interconnected global telecommunications.

Because network traffic is grounded-in the environments they extend through, in the rhythms of the bodies those who operate them, and in the patterned technics of their nonhuman milieus - the alteration of ground rhythms alters the speed of the system itself. If these typically balanced temporalities suddenly shift, the routinized temporality of an infrastructure is suddenly inflected by changes in its temporal milieu. I call these moments temporal irruptions: moments when the assemblage of temporal processes that enable network operation and network speed suddenly and radically changes the network. The fourth section of this essay charts how such irruptions along cable routes produce disruptions in the patterned time of the global network. Weaving through a set of breaks, disruptions, and irruptions in the cable network, I show how the elongated rhythms that keep networks safe can suddenly interrupt - and irrupt into—-the cable system. I argue that these irruptions direct our attention to the ongoing role of everyday, lived and embodied temporalities and the rhythms of cabled sociality, and the ways that infrastructures are "cyclical and repetitive processes of formation and transformation. ${ }^{n}$ As a complement to the hardwired temporalities that occupy much of this book, I call these soft temporalities, as they are the underbelly—a shifting and complex temporal substructure—of hardwired media and infrastructural times.

\section{Patterned Time}

In his well-known essay "Technology and Ideology: The Case of the Telegraph," James Carey demonstrates how the telegraph enabled the separation 
of physical objects from communication for the first time and, in doing so, was critical in the establishment of standard time, the transformation of futures markets, and the evolution of time contracts. ${ }^{7}$ Cable infrastructure, he showed, facilitated the production of modern, patterned time, and was an essential part of the temporal grid that restructured the industrial world. Undersea cables are the global links in this history, enabling the standardization of time on a global scale. An early function of undersea networks was to establish differences in longitude — which, as Richard Stachurski documents, "were literally a matter of time." ${ }^{8}$ One of the first uses of the transatlantic cable was to send star-transit timings that would help to determine longitude. The United States Coast Survey built temporary observatories at both ends of the cable, and between October and November 1866, clock signals were sent between the two locations on five different nights, "yielding the first directly-measured longitude of the dome of the U.S. Capitol west of the Greenwich Observatory: 5 hours 8 minutes and 2.22 seconds." ${ }^{9}$ The completion of the All-Red Line at the turn of the twentieth century - a British network that encircled the earth, was an infrastructure for the creation of Universal Standard Time. ${ }^{10}$ Today fiber connectivity via undersea cables enables the global coordination of digital time, and undergirds the precise operations of global positioning systems (GPS) among other digital operations.

Undersea cable systems, as was the case for telegraph systems generally, were perceived as annihilating time and space. This was true even within the cable industry. For example, in the cablemen's magazine, The Zodiac, many issues featured the final lines of Rudyard Kipling's "The Deep-Sea Cables": "They have wakened the timeless Things; they have killed their father Time." Although cable networks were understood to make time matter less, in actuality they substituted one form of temporal difference (in which temporal difference correlated with geographic distance) for another (a temporal difference that correlated with distance from infrastructure). In other words, the cable system produced a new set of temporal patternings in which elite users located in privileged positions felt synced with other elite users in privileged positions - they felt the distance between them less and less as temporal delay. In turn, cables introduced a new temporal structure in which those who did not have access to cabled systems or cabled information would more frequently perceived themselves as behind, delayed, and distant. In accelerating the production of a globalized temporality, whether through finance, news, or standardized time, the submarine telegraph also intensified a colonial temporal regime and was a means by which the Western empires were able to dominate the nineteenth-century timescape. 
Today's undersea cable networks, which carry $99 \%$ of transoceanic internet traffic, continue to restructure media temporalities in ways that parallel many of the telegraph's original impacts. In the world of highspeed trading, the cable system similarly both standardizes and accelerates machinic temporalities. This is especially true given the recent turn toward lower-latency cable systems. In network traffic engineering, latency is the amount of time it takes for a signal to make a round-trip between two terminals. Although the optimization of networks for speed has varied over the decades of cable laying, recent networks have been designed specifically as part of "low-latency" solutions. These have been developed to capture the market of high-frequency traders as well as for the range of computational practices that are dependent on speed-from cloud computing to the navigation of remote vehicles.

Take for example the 2015 Hibernia Express cable, which introduced a new low-latency path between New York and London-clocking in at 58.95 milliseconds. ${ }^{11}$ In 2017, the Seabras-1 submarine cable supplied Seaborn Networks with a "proprietary ultra-low latency (ULL) solution" (SeaSpeedTM) between the BM\&F Bovespa Stock Exchange in São Paulo, Brazil and Carteret, New Jersey. ${ }^{12}$ Geography and the shortest route isn't the only factor in latency - other technical factors include the refractive index of the fiber, the number of amplifiers, and the process of equalizing the fibers. ${ }^{13}$ Other traffic-related factors include network congestion, firewalls, and over-used routers. As one operator describes: "This also means that the integration for the land and sea routes must be taken into account, every interface can add latency."14 Achieving speed in the cable network never occurs merely through reduction of geographic distance, it often involves a consolidation of technical interfaces and a reconfiguration of social practice. Just as cable systems do not simply erase time, but re-pattern it differently for different publics, the production of the cable's temporal dynamics does not simply involve acceleration, but are a complex temporal and social operation.

\section{Grounded Speed}

What is often missing from these descriptions of cable speed are embodied practices that prop up acceleration-labor and social worlds assembled into fixed rhythms. Pulling its workers into a set of patterned times, the early telegraph system often sped up their movements and synced them to a machinic temporality. Some operators were required not only to meet a minimum speed, but also to ensure accuracy at this speed. As the poem, 
"Cablemen," describes cable work: "we work 'em like clockwork ... We must work them like lightning,/ Must work 'em pell mell,/ For it's through the thin cables/The Empire can tell/That all her Dominions and Children are well." ${ }^{\prime 5}$ H.F.B., the poem's author, describes the work of the cablemen as both "like clockwork" and "like lightening," drawing connections to the machinic and natural world to articulate the routinization and inhuman speed of the operator's body, all in service of the British Empire. The patterning of the men's bodies was structured by and in turn scaffolded British colonial expansion. Another cableman laments the lack of recognition for the men in the middle: "do you ever think how men/ Have worked the whole night through,/ And done the best within their ken/ to rush that Press-work through?"16 These descriptions of the operators' work echo throughout the cable magazines, with their details about late-nights and extended hours, immediacy to global events, the repetitive sounds of clock and telegraph, and the "dull routine" that propels the world forward. ${ }^{17}$ As Sarah Sharma writes: "Temporalities are not times; like continually broken clocks, they must be reset again and again. They are expected to recalibrate and fit into a larger temporal order." ${ }^{\prime 18}$ The temporality of the cable network was anchored in the ongoing recalibration of cable workers' embodied temporalities.

This patterning was not only machinic — keyed by the telegraph and modulated by cable. It was and continues to be social. In the telegraph era, regulations governing the rhythms of everyday life were intended to stabilize men's bodies, and as a result, the network's operation. One cable operator recounted his time at the Southport Station in Australia, where for probationers, "Church attendance once on Sundays was compulsory, and there was a 10 p.m. curfew ... The use of lamps in bedrooms was forbidden."19 Here the use of modern technologies of light were forbidden (even as candles were allowed) as part of the regulation of the men's nighttime activities. This ensured their adherence to a temporal pattern that would keep the cable system operational. In the telephone cable era, more complex technological systems would be deployed as a means of managing time and timing. For example, aboard the cableship Neptun, an internal CCTV network enabled "split-second timing" in cable laying operations_and for cable-laying operators. $^{20}$

The operators of the Eastern Telegraph System thus held together a number of overlapping temporalities in their own bodies. On one hand, they perceived their own role as an extension of the cable, annihilating temporal distance between Britain and the colonies. They both upheld its speed, with the actual speed of transmission reflecting the cumulative capabilities of 
all operators on the route, and saw themselves as the very first receivers: in 1926, one author remarked: "The work of a cable operator is a business of thrills and chills, though many imagine it to be a humdrum, mechanical occupation. You never know what is coming next. Cable operators have early news of world-shaking events." ${ }^{21}$ They occupied the subjective position, as with many other inhabitants along the colonial lines, in which a colonial temporal regime (the location of Britain as ahead) materialized in the technological extension of the cable system. At the same time, stationed at remote outposts around the world, as the anecdotes at the opening of this chapter testify, the cablemen were keenly aware of the continued temporal and geographic distance that positioned them at the fringes of empire. As they waited for supply ships, for letters and visitors to arrive, and for seasons to shift such that the environment would become passable, the cablemen's daily worlds shifted very slowly. The curfews, regulation of activity, and patterning of social exchanges in the cable station provided a slow and tedious rhythm of everyday life. Beyond this, a sense of slowness was produced in their extended posts at colonial hubs: the cable staff at any given location might remain in a given outpost for years. This strategic choice of the system managers would in turn help to establish continuity at the stations themselves.

Slowness, drag, and longer rhythms of movement did not inhibit the system - they constituted it. For the cablemen this sense of delay and stasis, perceived in their bodies and communicated through their internal magazines, intensified the sense that the environment surrounding them was "backward" or slow. At the same time they experienced an extraordinary temporal privilege. This sociotechnical and affective dynamic is what I describe as grounded speed. Today, grounded speed is the temporal substrate of undersea digital infrastructure, and can be best perceived in the processes of network construction, operation, and maintenance. The establishment of new cable networks continues to take years in planning, financing, and construction. Part of this process involves sending a survey ship to carefully document the prospective ocean route. In installation, the cable is coiled into a tank on the back of a cable ship, and dropped off the stern as the vessel crosses the ocean. The cable rests on the seafloor for the duration of its life, sometimes for decades without being disturbed. If the cable is broken, even if the ship is deployed to the fault location immediately, it might still take days or even weeks for the cable to be repaired, especially if the cable's owners are waiting to acquire permits for repair. All of these activities take time, and the duration of this process is extended by the slowness of marine transport, the need for careful operations, the tangled permitting 
process that occurs in some territorial waters, and the fact that once on the seafloor the cable cannot easily be retrieved. In other words, the difficulty of negotiating with the aquatic and political environment ground the system, making it relatively fixed once established, and discourage replacement. In turn, the ocean itself and the lack of human contact protects the cable, ensuring its continued speed.

Grounded speed is also present in the bodies of cable operators, though they are no longer confined to cable stations in remote outposts. Instead, many sit at screens in network operations centers (NOC), waiting for "alarms" to tell them what parts of the network need to be checked or repaired. The latest network operations technologies might release them from these screens, enabling the delivery of alarms via text message or smart phone. Whether in a NOC or not, the cable operators' bodies stay at attention and their movements are calibrated such that they can attend to the network at a moment's notice. Checking the network involves routine trips to a set of locations, including cable stations and beach landings. It involves regular maintenance and cleaning of these locations. While waiting for alarms keeps cable operators holding focus, ready to react at all times, ongoing maintenance is intended as a preventative measure, one that will forestall disruption through routine practices. In both of these cases, grounded speed exists in operation: signals keep flowing through the system at accelerated rates because of cable operators' repetitive motions, their familiarity with a narrow set of routes, and their relative stasis.

Another way that grounded speed exists in cable operation is in the relative stasis of the cable industry. Many people who work to construct undersea cable systems, whether in supply, marine operations, management, and even sales, have been in the industry for decades. In a recent presentation at the SubOptic conference, the triennial event of the subsea cable industry, analyst Kristian Nielsen described the problem of the "generation gap," and to prove his point, asked the members of the audience to keep their hands raised if they had been in the cable industry for more than twenty years. His audience, with many of their hands in the air, already understood his point: they had worked with the same set of people since at least the 1990s. While Nielsen and others have identified this as a problem in the industry - there are not very many younger members to take the place of the existing cable labor force-the lack of turnover and the ongoing stable presence of industry veterans grounds the system. Their continued practice ensures continuity of operation, availability of knowledge, and a vast archive of past negotiations with difficult social and environmental actors. The system stays intact precisely because of the lack of change in 
the cable world's social fabric. Repetition (whether social or machinic), prolongs interactions and slowness scaffolds speed.

\section{Temporal Irruption}

At the bottom of the Luzon Strait, relatively little changes. Sediment shifts and marine creatures move, but the cables that extend through in this narrow stretch between the Philippines and Taiwan stay in place. Cables might shift slightly on the seafloor, but they rarely travel great distances. The ocean is a stable and continuous context for cable systems.

In 2006, the ground suddenly shifted. The Hengchun earthquake triggered a subsea landslide. It was not the sudden movement of the earth's tectonic plates that severed the cables, but the subsea landslide that sent waves of sediment hurling down the seafloor. When accumulating slowly over time, sediment itself does not disrupt a cable — many systems are buried at their shore ends. But the shift in ecological temporality, the deviation from the existing pattern of subsea currents and sediment motion, irrupted into the temporal patterning of the cable system. Suddenly network traffic stopped across the cable. Users at various locations suddenly could not load webpages or send email. They were stalled, temporarily stopped by an irruption of re-patterned deep-sea time.

Temporal irruptions are not simply the moments when a system fails. They are indications that the ground that sustains infrastructure operation has fallen out of sync. They are moments when the shifting temporality of other phenomena - of ocean and atmosphere; of operators and inhabitants - alter the rhythms of the cable network and the internet as a whole. While undersea cables are largely secured from human interference in the ocean's depths, terrestrial fiber optic cables are regularly disrupted by ongoing projects of ground disturbance, especially digging along the cable route. Local construction projects, which involve the shifting and redevelopment of architecture, power lines, and water lines among others, often involve digging. This shift in an otherwise stable temporal patterning likewise irrupts into the network, causing a temporal disruption. In the ocean, storms have caused ships to put down anchor, stabilizing themselves in an otherwise tumultuous sea, but in turn severing cable systems. These are not mere moments of environmental interference, they are moments of temporal disjuncture.

In moments of irruption, network time is out of sync. The slowness, the cycles, and the patterns that had previously grounded the cable network then prevent the network from resuming at speed. Take for example this 
problem in the case of Arctic undersea cables. While cables always take time to repair, in the Arctic frozen ice can inhibit a cable ship's passage for a significant part of the year. If a cable breaks during this period, one operator reflects, "you need to sustain a fault for months sometimes." ${ }^{22}$ The rhythm of environmental phenomena, jarred out of sync with the network itself, no longer serves as a continuous shelter, a ground for the system. Instead, operators must find other ways-whether other cables or environments - to sustain network traffic and temporal operations.

\section{Conclusion}

Over the past decades, media studies scholars have documented the temporal regimes of network technologies, from the manipulations of infrastructural optimization and efficiency, to the emergent microtemporalities of digital systems, to planned social and technological obsolescences. This chapter shows that the system of submarine cables is an infrastructure that helps to prop up these temporal regimes, facilitating distinct and historically-specific patternings of time. It facilitates synced, standardized and universal time, but it has also propped up varied colonial, capitalist, and globalized temporal regimes. Looking beyond the hardware of cable systems, this chapter's study of network operations reveals the ways that all of these regimes depend on the syncing up of numerous human and nonhuman temporalities. Machinic time produced via cable systems is inevitably interlaced with social and environmental time - the reconfiguration of machinic time is both dependent on the organization of the temporality of labor and seasons, and exists within its limits. Undersea cables are relatively stable infrastructures compared to most digital systems, and this is in part due to a multitude of extended and embodied rhythms: from the operators standing by to the social fabric of cable landing stations and management centers, where the goal is always stasis.

In these sites, the human-machine interface is always also a temporal interface, where the patterned time of cable systems is enfolded in a multiplicity of other time-scales and temporal practices. If technical systems hardwire media and social practice in a variety of ways, these systems are nonetheless inevitably tethered to soft temporalities. Soft temporalities are those that are variable, exist beyond the machinic, and emerge in embodied practice. If hardwired temporalities encode temporal regimes and govern activity, the soft temporalities of digital infrastructure comprise both its base layer and its most vulnerable context. 


\section{Notes}

1. Phillip Vannini, "In Time, Out of Time: Rhythmanalyzing Ferry Mobilities," Time \& Society 21, no. 2 (2012): 241-269.

2. "The Gooney Clarion," The Zodiac: The Journal of the Submarine Cable Service, 6 (June 1912-May 1913): 61-72; "The Lonliest Station," The Zodiac: The Journal of the Submarine Cable Service 10 (June 1917-June 1918): 109.

3. "The Trail of Lonesome Midway," The Zodiac: The Journal of the Submarine Cable Service 9 (June 1916-June 1917): 116.

4. Sarah Sharma, In the Meantime: Temporality and Cultural Politics (Durham, NC: Duke University Press, 2014).

5. Paul Virilio, Speed and Politics (Los Angeles: Semiotext(e), 2007); Jonathan Crary, 24/7: Late Capitalism and the Ends of Sleep (London: Verso, 2013).

6. Gabriele Schabacher, "Time and Technology: The Temporalities of Care" (this volume).

7. James W. Carey, "Technology and Ideology: The Case of the Telegraph," Prospects 8 (1983): 303-325.

8. Richard Stachurski, Longitude by Wire: Finding North America (Columbia: University of South Carolina Press, 2009), 15 .

9. Trudy Bell, "The 'American Method': The 19th-Century Telegraphic Revolution in Finding Longitude" (work-in-progress paper presented at the IEEE Conference on the History of Telecommunications, St. John's, NL, Canada, Friday, July 27, 2001), 6.

10. Notably, Sandford Fleming, the Canadian who had proposed the adoption of Universal Standard Time, was also the principal proponent for the AllRed Line.

11. TeleGeography, "Trans-Atlantic Network Latency Reduced," The Broadcast Bridge, October 9, 2015, https://www.thebroadcastbridge.com/content/entry/3988/trans-atlantic-network-latency-reduced.

12. Seaborn, "Spread Networks and Seaborn Team Up to Provide SeaSpeed ${ }^{\mathrm{TM}}$ : Brazil's First Dedicated Ultra-Low Latency Subsea Route," SeabornNetworks. com, May 8, 2017, https://seabornnetworks.com/spread-networks-and-seaborn-team-up-to-provide-seaspeed-brazils-first-dedicated-ultra-low-latency-subsea-route/.

13. Alasdair Wilkie, "Low Latency Cables" (paper presented at SubOptic Conference, Paris, 2013), https://www.suboptic.org/wp-content/uploads/2014/10/NO02_Poster_105.pdf.

14. Wilkie, "Low Latency Cables."

15. H. F. B, "Cablemen," The Zodiac 9 (June 1916-June 1917): 211.

16. Crispian, "Cable (K)nights," The Zodiac: The Cableman's Paper 8, no. 85-96 The Zodiac: The Journal of the Submarine Cable Service, vol. 9 (June 1916June 1917): 44 .

17. R. M. M., "The Man at the Key," The Zodiac (March 1917): 227.

18. Sharma, In the Meantime, 7 . 
19. "Early Days at Southport," State Archives, Southport Australia, Premiers Papers Re: Pacific Cable, 6.

20. “Candid Camera ... NEPTUN Version!" Underseas Cable World: A Bi-monthly report by U.S. Underseas Cable Corporation 1, no. 3 (Feb-March 1967).

21. George E. Clarke, "Cable Thrills," The Zodiac: The Submarine Cable Service Paper 17, no. 210 (1926): 316.

22. Hector Hernandez, "The Push for Polar Projects: Challenges and Remedies Impacting the Design and Implementation of Arctic Submarine Cable Systems" (paper presented at SubOptic Conference, New Orleans, April 11, 2019).

\section{About the Author}

Nicole Starosielski, Associate Professor of Media, Culture, and Communication at New York University, is author of The Undersea Network and Media Hot and Cold, and co-editor of Signal Traffic: Critical Studies of Media Infrastructure, Sustainable Media: Critical Approaches to Media and Environment, Assembly Codes: The Logistics of Media and the "Elements" series (Duke University Press). 



\title{
10. Unruly Bodies of Code in Time
}

\author{
Marisa Leavitt Cohn
}

\begin{abstract}
This chapter examines how debates about the (im)materiality of software comes to inhabit the practices of software engineering work who manage the temporality of obsolescence and its entanglement with their own careers, language proficiencies, and expertise during the lifetimes of systems they develop or maintain. It describes how bodies of code endure materially in ways that exceed their formal understanding, revealing how the hardwiring of temporality into digital systems takes place through a moral economy of software work that devalues of code as it ages and obsolesces. The habitus of the programmer is set within a disciplinary regime that sustains the imaginary of software as immaterial, infinitely flexible and malleable in spite of routine encounters with its material recalcitrance.
\end{abstract}

Keywords: Maintenance, software work, temporality, legacy systems, obsolescence

That which decays is not software.

This is a claim I encountered after giving a presentation of my work to an audience of colleagues at my university that included designers, social scientists, and engineers. I had spoken about the concept of "convivial decay," a term I have used to describe the achievement of engineers working to maintain aging and obsolescing infrastructure. ${ }^{1}$ In the talk I had spoken about the different forms of decay that engineers encounter-from instruments shorting out, to programming languages reaching end of support, to obsolescing standards for software change management. After the talk, one of my colleagues, a software engineer who also provides IT support to some of the university labs, explained to me that while intriguing, I had made an error in claiming that software decays. "Software doesn't decay," 
he said, "everything but the software decays." I understood his contention. "Sure," I replied, "but then, where is this software? Where does this thing that does not decay exist?" He laughed at my question, dismissing it as a philosophical concern best left for an afterwork conversation.

This disagreement reflects a debate between materialist and immaterialist views on the nature of software that has animated much discussion among scholars of computational media. We have, for example, the assertion from Kittler in his well-known essay "There is no software," that "all code operations, despite their metaphoric faculties ... come down to absolutely local string manipulations and that is, I am afraid, to signifiers of voltage differences." ${ }^{2}$ On the other hand, we have claims that what is most essential to the nature of software is its "operativity"3 or "executability," an abstraction that exists outside of the particular instantiations of hardware and software that "coalesce" in order for the code to function. ${ }^{4}$

Rather than stake out a position within this debate, my aim in this chapter is to ask how this debate comes to inhabit the discourses and practices of software engineering work. In my ethnographic fieldwork, programmers and software engineers inevitably deal with material instantiations of code, particularly in cases where they are working with long-lived systems running on legacy software. Aging software systems require engineering methods that are similarly forensic and archaeological as the methods of media scholars unpacking historical or residual media. Yet at the same time, these encounters with the materiality of code, while commonplace, still seem to rupture with regularity a strongly held belief in software as immaterial, as that which does not decay.

Materialist approaches to software are often adamantly reliant upon engineering know-how. Kittler wishes to give engineering knowledge primacy as the proving ground for theorization. ${ }^{5}$ Others in software studies have debated whether you can theorize software without being a proficient coder. And, as Dourish points out, when technologists work with code, the material constraints of software systems are unavoidable. "Programmers understand the ways in which digital structures can resist their will, every bit as much as clay, wood, or stone. ...Materiality - the nature of the substrates and the properties that constrain and condition the designerly encounter-is at the core of each experience." ${ }^{\prime 6}$ If engineers know this materiality of software so well, why does an immaterial view have such staying power? Why and how is an immaterial view of software privileged and sustained despite the obviousness of its materiality?

What I suggest here is there is an overlooked temporal dimension to this tension between software as material/immaterial. As software ages, its 
materiality becomes more and more unruly, more recalcitrant. A new line of code just written by a solitary coder/theorist might be easy to change, but once embedded into the complexity of interoperating systems being maintained by multiple generations of engineers, such changes become the object of much organizational scrutiny and negotiation. Bodies of code are increasingly bound up in the contingencies of historical organizational decisions, material constraints of available technologies, as well as the careers of those maintaining the code.

Through empirical material drawn from ethnographic fieldwork with a large engineering organization, I argue that what is at stake in this tension are the valuations of different forms of computational work over others. The negotiations that take place in managing aging software are not only a matter of securing computational systems from disastrous changes; they are also a matter of how engineers manage the temporality of obsolescence and the entanglement of their own careers, language proficiencies, and expertise with the lifetimes of systems they develop or maintain.

If we were to track the biography of a software system over time, we might observe an unfolding process of its materialization that mirrors in many ways a process of decommodification described by Kopytoff.7 As software ages it becomes increasingly "singular, unique, and unexchangeable" and embedded into the social milieu of the organization. Those who want to commoditize their expertise must then detach themselves from the concerns of particular bodies of code and their accidental materialities, and align with more universal, timeless ideals of code as immaterial. It is through this marginalization of material concerns, I argue, that an immaterial view of software is sustained within the moral economy of software work.

The following vignettes are taken from ethnographic fieldwork conducted in 2010-2011 with the Cassini mission to Saturn at the Jet Propulsions Laboratories. The case offers an extreme example of engineers working with a long-lived infrastructure built to maintain and command a spacecraft navigating in orbit around Saturn, that was built and launched in the 1990s. While the hardware and software on-board the spacecraft is over a decade old, so too is much of the software used to command it (known as the "ground system") because it must remain compatible with the spacecraft. Nonetheless, there are plenty of pressures towards upgrade and adaptation on the mission, due to programming languages reaching end of support, new software management methodologies in vogue at the lab, as well as down-sizing of personnel requiring the "leaning" of software work.

These vignettes are snapshots from within the lifetime of the mission that capture the entangled biographies of engineering careers and bodies of 
code. $^{8}$ As Kopytoff notes, a cultural analysis of the biographies we tell about objects can reveal a "moral economy that stands behind the objective." $\mathrm{We}$ can hear in these vignettes, evaluations of what constitutes a successful career or a good software system. What is negotiated in the stories told to me about the mission and its software systems are the attachments, both economic and affective, that are formed with software over time. In the discussion that follows the vignettes, I unpack further how time and code are figured together within these stories, and how particular affective attachments to code are pathologized and devalued.

\section{Vignette 1: "Gripping the Casket"}

William is a navigator who has joined the Cassini mission quite recently but who loves the work, which he says is the most exciting work of navigation going on at the Lab. No other mission has as much navigation work to be done on an ongoing basis, and he associates much prestige with the fact that mission still knows the location and trajectory of its spacecraft with such precision and is still flying successfully after so many years. But William also sees that many of the ways of doing navigation work at the mission have become entrenched and is eager to get on board with new software developments at the Lab-to harness the "power" of new software.

When I arrived and for full duration of my time in the field, the navigation team was struggling to migrate to a new software system, and so were running in what they called "parallel ops"-flying the spacecraft with two software systems at the same time. These software tools are used to determine and analyze the spacecraft's current vector in space (i.e. where it is and where it is currently headed) and to plan upcoming maneuvers (for example "trimming" an orbit by firing thrusters). ${ }^{10}$ Cassini was a new "customer" to a multi-mission software system called MONTE that had long replaced the legacy system on all other missions at the lab. Cassini was the only mission coming on board to the tool after launch and during the long-term maintenance and operations phase of the work, after the team has built up over a decade of experience with the spacecraft's behavior and the tools used to navigate it.

William explained that for many of the old-timers, navigators who had worked on the Cassini mission since its launch, they could not see the value of the new system. For one thing, he explained, there is the question of why you would try to learn a new programming language when you are "ten years deep" in another. He also discussed the risk of doing too much 
work to adopt and adapt a new software tool. Working on software is "not our job description" and does not have much visibility within the organization. But despite this William had decided to develop an interface with the new software, through the creation of a new design mark-up language (an Interface Description Language, IDL), that would accommodate the mission's need to run multiple analyses in parallel by adopting the same naming conventions used to keep the various potential solutions distinct. Over multiple meetings he tried pitch the new language to his own team and to lab-wide meetings of navigators with the hopes that this would allow his team to interface with the new software while maintaining older ways of working.

William was particularly sensitive to the fact that navigation work can change dramatically in nature depending on the software tools one uses. He views working on software as a way of showing deep care for the mission and the work that they do, but also a risk to one's career. Aligning to software work can make one indispensable if you are the only person who knows that tool, which could be bad in the long run if some other more exciting mission comes up. It also puts you at risk of becoming a software person and being seen as someone to ask to write code rather than to design a mission tour.

"If anything it can be harmful for you. They hired me because I know astronautics, they don't care about my coding. I could become the code guy and be downgraded to writing scripts for others. Instead of oh William is a $\mathrm{PhD}$ rocket scientist, so have him design the maneuver, [it could be] oh no, William is good for coding, put him there. So sometimes it is to your own detriment to write good code."

Yet for William, working with the new software was also a matter of distancing himself from the legacy systems in operation at Cassini that are no longer relevant. At a meeting with other navigators at the lab demonstrated his new interface design language in a presentation on the unique challenges faced by the Cassini mission which he outlined as a set of ten "pathologies" within the legacy navigation database led to wasted effort. ${ }^{11}$ Through this presentation, William works to demonstrate to his fellow navigators at the lab that even within the context of a mission that is perceived as a "dinosaur" he is capable of staying innovative and up to date in his ways of thinking about navigation work. By positioning himself against the legacy software to his broader professional network at the lab, William made sure that while he was aligned to the mission system, he was not aligned to the software code in the same way that his colleagues were.

His failures to get his team on board with the new design language aside, his ability to narrate his own practice in the terms of the current 
technoscientific paradigm by pathologizing the legacy software aligned him to new disciplinary regimes, to such an extent that he was even called out for "selling out" by one of his colleagues. Yet from his perspective, William sees some of his colleagues as making the wrong kinds of alignments to the legacy software, saying that they are "fearing the corpse but gripping the casket."

\section{Vignette 2: Surfacing a bug}

Another navigator, Chris, explained to me that he came to the Cassini mission fresh out of his masters degree and found himself caught off guard by the reality of his engineering work - being "thrown into the deep end of [programming in] Unix." Having had no introduction to it in school required him to make a "big leap" to work in a space with so little of the programming skills he needs everyday. He confessed to me that due to this lack of experience with he "seem[s] to attract software bugs ... or weird problems." He attributed this to the idiosyncrasies of his work, that he might have configured something ever so slightly wrong or done something a slightly different way. And then "all of a sudden," he tells me, "I get a bug." "[And all the] old-timers are in my office staring at me wondering how could you do that?!" He gives me an example of how one time, he was running a small test on a file through a program in his own file space when suddenly he modified an actual operations file that he should not have access to. "Technically I wasn't even supposed to have permission to edit [it]. And yet here I am doing my own little work ... And well people were starting to panic, wondering ... why is this input looking like this? how is he able to do it? Is the system not secure? I ran a little thing on the side and suddenly I'm corrupting our operations ... and it ended up being ... just a weird flaw in the legacy file."

Chris seems simultaneously a bit abashed but also in awe of the fact that by virtue of being new and knowing less and doing something "wrong" or at least off the normal path he actually lead to a kind of discovery of an anomaly hidden in the depths of the software system, something no one knew was there. "I'm like trying to follow everything [they're saying]. I actually learned a lot that way. And so did everybody else." He laughs and explains that engineers much more experienced than him "the old timers" were standing around his terminal saying "I didn't know you could do this or I didn't know you could do that." "And I just did it," he adds.

In fact, everything about the way Chris explained this story came across as a kind of confession, as if he were speaking in a way that he shouldn't. His 
voice lowered to a whisper and his tone implied both an apology for how things work on such an old mission, and an appreciation for a system that has been around for so long. He laughed uneasily as he told me this story and added that when he hears about the legacy code "I hear people say 'the legacy code has been around for more than 40 years'" he thinks about how it accrued over time as a kind of "patchwork" one that spans many people's careers, many hands having touched it over the years. And while he agreed that the new ways of developing code, these newer programming languages and platforms, do make code more maintainable, more evolvable, flexible and extensible, at the same time this software being developed today, "I wouldn't say, I can't say that this software will last another 40 years. Who knows how long it is going to be relevant ..." When I ask if after he surfaced this bug in the system, were they able to fix it. "Not really, but [we could] just be more aware of [it]. We call those 'features.' When it is something that you can't change and is just the way it is. It's a feature. Like we have features," he says, as he gestures to his face.

\section{Vignette 3: Return Tour of Duty}

Reza is a software developer who began working at the lab in 1995 and soon after began working on the Cassini mission in 1996 when they were nearing their launch date. Like Chris, Reza took an apologetic tone when explaining to me what he finds inspiring about working on this project. Over the duration of his career, he had moved around a lot from mission to mission, and it was only recently that he had been brought back in to work on the Cassini mission. He had been tasked with jumping in to get a piece of code working again that had broken when the person who wrote it had left. I had asked him about what he was working on. Like Chris he could not believe I would be interested in work he was doing with legacy code. But, he explained, that is work he enjoys doing, picking up some code written by other people. "Half of my career has been doing that actually, that is just part of software development. You always enter a project. You're seldom aligned [so that] you're there right at the beginning... . Or," he continues, laughing, "there's always, even if you are right there right at the beginning, some projects inherit code from other projects."

I asked Reza what he likes about working with legacy code and he points to a few things. First, he likes what he calls the "detective work." "It is a matter of going through the code and trying to decipher what was the intention, what was he trying to [do]? What was the product he was trying to create 
[and] why didn't it get created. Because [there's] the trail. You can see what he was trying to do." He also liked that working with old software held nostalgia for him. Returning to a mission where he worked years back as a young engineer, he encounters some of the same systems that were written at those times. He described coming back to Cassini as a return tour of duty, and it was in a tone of duty and care that he spoke about the mission as one that provides a good working environment precisely because of the care that has been put into it over so many years by so many engineers. And it was also the "diversity" of a project where you might work one day in something like a really "old X/Motif GUI that is not refreshing properly ... and from there I go to something like Drupal which I knew nothing about yet... It's just constantly changing." In contrast, he said that working on some of the newer missions made him feel like a programmer that is just a replaceable cog in the system. He said the work is "cookie-cutter"-the work broken down in a Fordist like manner making his work repetitive and his own prior experience irrelevant.

\section{Discussion}

In these vignettes, we see how software work involves affective attachments, as well as detachments, from ways of working with code. In William's story, he admonishes his colleagues for forming attachments to particular ways of working with obsolescent software systems in ways that might be detrimental to their careers. On the other hand, he is called out for being too careless in how he cuts ties from old ways of working with software, and too self-interested in how he capitalizes on a narration of the mission's software systems as backwards and pathological. Meanwhile, in Chris and Reza's accounts of the Cassini software, there is also an apology for the forms of attachments one makes to legacy software. For Chris, as a young engineer, he knows he is not supposed to find novelty and intrigue in a system that is so old - a "well-oiled machine." Like William, he hints at a more proper and expected allegiance to the newer systems and ways of working. He explains that the new systems are indeed better, parroting the terms "extensible, maintainable, evolvable" in a voice that signals ideological-speak.

Chris's experience working with legacy systems has disrupted a particular ideological attachment that he has encountered in the broader discipline of software engineering, one that overvalues current systems and their anticipated futurity. What does it mean, after all, for a system to be more 
maintainable than one that has been maintained for over 40 years? Systems are durable, not because of some attribute of the programming paradigm in which they arise, but simply by virtue of people contributing to keep it going. Taking this even further, Reza's appreciation for legacy code (one that he consistently apologized for) suggests that what is gained with newer systems is not so much a cure-all against obsolescence, but a way to ensure that histories are continuously truncated. If the new software is more "maintainable" it is in the sense that has become increasingly commoditized. But in order for code to act as a commodity it must also be effaced of the historicity that gives Reza pleasure in working with legacy code.

These stories reveal competing valuations of software, as well as of ways of knowing and becoming affectively attached to software. One can care too much for code in ways that are detrimental to career or one can be too careerist in cutting ties from old code. A paradox presents itself in the idea that one can be overly attached to the material concerns of software, yet it is clear that such attachments are what keeps a system going, since the code remaining operative relies on people who maintain proficiency and understanding with legacy languages. It is also clear that while there are competing valuations, there is one that dominates, inflecting the awe and richness that both Chris and Reza see in the legacy code with apology and irony. This speaks to the power of the disciplinary regime that governs proper attitudes of the coding subject.

In thinking through these affective ties to old code and how they were disavowed by Chris and Reza, I was reminded not only of Foucault's discussion of governmentality but also his essay on Technologies of the Self (Foucault 1988) in which he asks "through what operations we work upon our bodies and souls, thoughts, and conduct ... so as to transform themselves in order to attain a certain state of ... purity, wisdom, perfection, or immortality." While Foucault is talking about the techniques applied to the body, we can easily extrapolate to techniques applied to the body of code, as I have done so here by adapting his questions for my purpose:

- If one wants code to behave rationally and if we want to regulate code according to certain principles of what makes code rational, what part of code or ones work of coding should one renounce?

- How do individuals effect by their own means or with others a set of operations on the body of code, on their conduct towards the code, so as to transform the code into a state of perfection?

These questions apply quite readily to the context of software development as seen in the vignettes above. They help to highlight two modes of knowing 
and attaching to code. The mode of innovation in which one does not align too closely to any aging or obsolescent system. And the mode of maintenance and care in which one understands code materially through its longevity and an attachment to the past as a source of insight. In the first code is commodified, current, and promises longevity through a renunciation of obsolescence. In the latter, the code itself is a rich tapestry or patchwork that binds together many different careers that sustain the body of code's longevity - that is a way of knowing code that is rooted in continuity rather than in a regime of anticipation and futurity. ${ }^{12}$

Others have pointed out that software is bound up with philosophical commitments and morality. ${ }^{13}$ What I have highlighted here is how the moral economy of software work also applies to its aging and obsolescence. As John Durham Peters states, "obsolescence always raises moral questions about the subjects and objects we neglect"14. In the aging and obsolescence of software, time and code are configured together. In long-lived systems, particular temporalities of work must be maintained in order for the system to remain vital, and likewise a system can "fail" for lack of those who know how to program in older languages. At the same time, valuations of software also shift as it ages. Old software resists commodification, displays too much personality, bugs becoming features. In software engineering communities, legacy is considered a derogatory word, referring to code that has stuck around too long and become heavy. Old software is pathologized for being mired in the past, and those who care too much for it are as well. Even the terms used in software engineering to manage aging systems like rot and grime ${ }^{15}$ suggests a relation to the abject. At the same time, newer systems and methods are adopted with a rhetorical promise of eternal youth, as the solution that will never age.

A materialist approach to software contends that the "trope of immateriality" is both analytically weak, smoothing over technical complexity, and ideological in suggesting that digital systems liberate us from the historical and material contingencies of other media. ${ }^{16}$ Drawing on Hayles, Blanchette suggests that this trope is part of an ideological project that has as its underlying "fundamental assumption, that informational patterns (including human consciousness) are ontologically superior to their (accidental) material instantiations (including the human body). ${ }^{\prime 17}$ This same ideology inhabits software programming work, in part because it is a feature of obsolescence that an engineer can liberate himself from the contingencies of the at-times arbitrary material conditions of the past by choosing to write new code rather than maintain the old. It is thus also the habitus of the programmer set within these disciplinary regimes that 
forms part of the mundane apparatus sustaining the imaginary of software as immaterial, infinitely flexible and malleable.

\section{Conclusion}

Ernst has claimed that "temporal transcendence of materiality is a faculty of operative media" in the sense that there is such a "tight coupling" between elements of hardware and the material knowledge of them, that they act as a kind of time machine. When technologies are reactivated at a later time they might require modification to get them working, but this is done in such a way that "unleashes" the "latent presence" of their operations. ${ }^{18}$ What he points to is the way that the "operation" itself endures in ways that the material instantiations do not. This makes sense for understanding the transience of software's material instantiations and how older systems become a repository of memory of older ways of working (as they did, for example for Reza). However, this fails to make sense of how bodies of code endure materially in ways that exceed their formal understanding.

As software ages, it can become no longer executable because of some perturbation in the many interdependencies of a body of code and the people who operate it. A person moves off the project, or a subsystem fails, and the organizational memory is no longer there, if it ever was, to hold the system together. This suggests that legacy software might become unable to temporally transcend its materiality simply because the tight coupling of hardware and knowledge has been lost. But it also, I think, betrays a presentist bias in how we philosophize the nature of software that leaves aside the problem of temporalities of software's aging and obsolescence. That is, even when we examine the historicity of computational media, our analysis privileges how this historicity is made manifest in the present. What about the latency of a bug in 50-year old software, as an aspect of its (in)operativity, that is only revealed over the long lifetime of software. The bug, which might also become a feature, is a constitutive part of software as both a material and formal object.

As I have hoped to show, the hardwiring of temporality in digital systems, is at least in part performed through the valuations of software as it ages. Such valuations arise not only with regard to the performativity or executability of code, but also in regard to the multiple biographies of any body of code, the many lives and careers it has touched or sustained. In a culture where as code ages and becomes more mired in material concerns, the work of maintaining it is devalued and even pathologized, privileges accrue to 
the software yet to be written. This ethos shapes the attachments and moral commitments of engineers to competing valuations of maintenance and innovation. As in rubbish theory, legacy code is that which is not yet thrown away but is durable despite its devaluation and troubles the moral economy of software work. ${ }^{19}$ It is in this duration of unruly bodies of code in time that the ideology of "immateriality" lives.

\section{Notes}

I would like to thank Axel Volmar and Kyle Stine for organizing the series of workshops on Hardwired Temporalities as well as the participants in those workshops for their feedback on this piece, particularly Gabriele Schabacher, Geoffrey Bowker, and Alexander Monea for their in-depth comments. I also appreciate input from Nanna Thylstrup and Mace Ojala who significantly advanced my thinking in later drafts.

1. See Marisa Leavitt Cohn, "Convivial Decay: Entangled Lifetimes in a Geriatric Infrastructure," in Proceedings of the 19th ACM Conference on Computer-Supported Cooperative Work and Social Computing (ACM, 2016), 1511-1523.

2. $\quad$ Friedrich Kittler, "There Is No Software," Stanford Literature Review 9, no. 1 (Spring 1992): 84 .

3. Wolfgang Ernst, "Micro-Dramaturgical Temporalities of Media Theatre: On the Difference Between Performative and Operative Reenactment," in Performing Arts in Transition: Moving between Media (New York: Routledge, 2019), 55-68.

4. Rob Kitchin and Martin Dodge, Code/Space: Software and Everyday Life (Cambridge, MA: MIT Press, 2011).

5. $\quad$ Friedrich Kittler, "Real Time Analysis, Time Axis Manipulation," Public Culture 13, no. 1 (2017): 1-18.

6. Paul Dourish, The Stuff of Bits: An Essay on the Materialities of Information (Cambridge, MA: MIT Press, 2017), 6.

7. Igor Kopytoff, "The Cultural Biography of Things: Commoditization as Process," The Social Life of Things: Commodities in Cultural Perspective 68 (1986): 64-91.

8. Steven J. Jackson, David Ribes, Ayse Buyuktur and Geoffrey C. Bowker, "Collaborative Rhythm: Temporal Dissonance and Alignment in Collaborative Scientific Work," in Proceedings of the ACM 2011 Conference on Computer Supported Cooperative Work (ACM 2011), 245-254.

9. Kopytoff, "Cultural Biography of Things," 64.

10. There are many reasons that the navigation team found it difficult to switch to the new software system. In contrast to the legacy code, which was developed "in house" at the Cassini mission, adapted from earlier missions to suit Cassini's needs, the new software system was developed by a new multi-mission software team at the lab that maintains a code base 
and unit tests to keep the software working for multiple missions at once. While other missions can provide requirements for the system and work in advance of launch to test out the software, Cassini was the only mission expected to change to the new software mid-flight. Not only was the new software producing different results from the legacy software (literally plotting different vectors for the spacecraft's current trajectory), but each time the new software was successfully tweaked to produce results for Cassini's navigation team, some new update to the software would break the team's in-house tools. Many of the initial issues discovered in transitioning to the new software came down to the material instantiation of the code. For example, the new software was written in Python, which stores variables differently than the legacy code, in terms of how it handles truncation of decimals. These types of fixes were relatively easy to deduce, but never fully brought the tools into perfect congruence. The new software also ran very slowly, something that is less of an issue for missions that design one set of navigation maneuvers and parameters for a successful launch and entry into a planet or stable orbit. The Saturn mission, however, had to consider multiple maneuvers to execute on a weekly basis.

11. Many of these so-called pathologies came from inheritances from even earlier missions that had done most of their navigation calculations by hand. The legacy software draws on what was originally a digitized version of paper files, which unlike a modern database did not have a clear separation between form, syntax, and content. What used to be considered a "view" of the database (a print out) is what is now treated as data.

12. Adrian Mackenzie, "Programming Subjects in the Regime of Anticipation: Software Studies and Subjectivity," Subjectivity 6, no. 4 (2013): 391-405.

13. Paul Dourish, Where the Action Is: The Foundations of Embodied Interaction (Cambridge, MA: MIT Press, 2004); Christopher Kelty, “Geeks, Social Imaginaries, and Recursive Publics," Cultural Anthropology 20, no. 2 (2005): 185-214; E. Gabriella Coleman, Coding Freedom: The Ethics and Aesthetics of Hacking (Princeton, NJ: Princeton University Press, 2012).

14. John Durham Peters, "Proliferation and Obsolescence of the Historical Record in the Digital Era," in Cultures of Obsolescence. History, Materiality, and the Digital Age, ed. Babette B. Tischleder and Sarah Wasserman (New York: Palgrave Macmillan, 2015), 79-96.

15. Clemente Izurieta and James M. Bieman, "A Multiple Case Study of Design Pattern Decay, Grime, and Rot in Evolving Software Systems," Software Quality Journal 21, no. 2 (2013): 289-323.

16. Jean-Francois Blanchette, "A Material History of Bits," Journal of the American Society for Information Science and Technology 62, no. 6 (2011): 10421057, https://doi.org/10.1002/asi.21542.

17. Blanchette, "Material History of Bits," 1044.

18. Ernst, "Micro-Dramaturgical Temporalities of Media Theatre."

19. See Michael Thompson, "Time's Square: Deriving Cultural Theory from Rubbish Theory," Innovation 16, no. 4 (2003): 319-330. 


\section{About the Author}

Marisa Leavitt Cohn is an Associate Professor in the Technologies in Practice group at the IT University of Copenhagen where she co-directs the ETHOS Lab. She combines anthropological and design-oriented approaches to the study of human computer interaction focusing on temporalities of work in long-lived infrastructures and legacy system maintenance. 


\title{
11. Screwed: Anxiety and the Digital Ends of Anticipation
}

\author{
James J. Hodge
}

\begin{abstract}
This essay discusses anxiety as the paradigmatic malady of the present and its correlation with the rise of always-on computing. Discussing anxiety as "an expectation emotion," the essay notes the ways in which always-on computing has outsourced futurity to opaque computational processes. This essay asserts that the latter fuels the former. However, all is not lost. Through the analyses of three online texts-an artist's clock, a meme, and a YouTube video - the essay argues for a view of this situation that recognizes the pleasurable and resolutely social dimensions of anxiety. Discussion of these texts elucidates the dynamics of anxiety in always-on computing and challenges the assumption of anxiety as an individual problem.
\end{abstract}

Keywords: anxiety, new media art, social media, memes, death, phenomenology

This essay is about anxiety and how we deal with it. It's about anxiety as a felt problem or condition specific to the historical present of post-industrial cultures in the twenty-first century, which are characterized by the saturation of lived experience by always-on computing, or the milieu of smartphones, social media, and ubiquitous wireless networks. Anxiety, we often hear, seems to be the distinctive malady of always-on computing. ${ }^{1}$ Of course, anxiety is not new. Yet as the activist collective called the Institute for Precarious Consciousness (IPC) theorize, anxiety takes on a newly general character today arising from neoliberalism's "obligation to be communicable." To build on this formulation, always-on networks constitute the infrastructural conditions of possibility for this "obligation," where 
the obligation to be communicable involves a felt pressure and pleasure of being available or "always on" to networks by virtue of the sheer fact of carrying a smartphone and being enmeshed in networks. This essay, then, seeks to elaborate the relation of anxiety as an "expectation emotion" to always-on computing, especially for the ways it follows from the outsourcing of future-oriented experience (prediction, anticipation, etc.) to machines operating beyond lived experience. ${ }^{3}$

The essay also follows from a second issue identified by the IPC. Anxiety is almost always viewed as a personal or individual problem. In seeking to combat the deleterious and deflating effects of anxiety today, the IPC recommends understanding anxiety as a social phenomenon. I heartily agree with this prescription. To contribute to this project, I want to describe and theorize not only the ways in which anxiety correlates with always-on computing, but also the ways we are already managing anxiety as a collective problem. I argue that the task of describing anxiety as a major dimension of the lived experience of always-on computing requires attending to the circulation of anxious objects, which generate a palpable sense of anxious fun. Understood as networked aesthetic objects to be shared, they trouble any strictly individual notion of anxiety and open up the social dimensions of anxiety today. My discussion analyzes three anxiously fun objects: a clock designed by an artist, a meme, and a viral video.

Start the clock.

Available on the online gift shop for Chicago's Museum of Contemporary Art at the discounted rate of $\$ 70.98$ (marked down from $\$ 285 !$ ), designer Brian Eaton's Memento Mori Clock looks like a handsome, walnut-encased alarm clock of modernist yesteryear. Its red and black digital display does not tell the time, however, but rather how much time you have left. An explanatory text reads,

Memento Mori serves as a reminder that all things must come to an end. Upon turning the clock on, you are prompted to enter some basic information about yourself. From there it pulls data from the World Health Organization to establish a countdown, in minutes, to your inevitable demise. At zero the clock will commemorate your life by playing a song, as a final reminder of your time here on Earth. This song can only be heard by those that make it to zero, as it's impossible to cheat the countdown. ${ }^{4}$

Is this a joke? While conceptually intriguing it's difficult to say who would be willing to participate in a countdown to her own death (maybe that's why it's available for $75 \%$ off?). When I have shown people this item they 
typically gasp, laugh, and exclaim along the lines of, "oh my god!" When I half-seriously suggest that I am thinking of buying one, their tone quickly turns serious. I must not buy one, they insist, sometimes even demanding that I promise not to buy it. The very idea of this clock, they say, makes them intensely anxious.

Why do my friends so discourage me from buying this clock? They say the idea of the clock makes them anxious but I really think it's the idea of my having it. What difference would that make? Having or owning a clock might do a few slightly uncomfortable things. Owning it might risk taking on the anxiety the clock generates as my own. In effect, it might become an extension and self-serving ratification of my anxiety, the anxiety that helps to make me me and distinguish myself from others. I hope I don't cheapen the felt impact of anxiety when I note that anxiety today sometimes appears as rather precious - distinguishing even as it distances. This certainly feels like part of the problem. Relatedly, owning the clock may diminish the clock's power to produce a social bond instanced by the common exclamation of "oh my god!" elicited precisely by sharing its image over text message, email, social media, etc. Ownership risks taking it out of circulation as a way to feel anxiety as a social feeling. I believe that is the real danger my friends object to when they demand that I not buy it. If I owned this clock, I don't think I could really share it again, at least not in the same way as I do when I don't actually own it but just want to say, oh my god look at this. Put more simply, the stakes I see this object opening up have to do with the nature of togetherness set against the background of networked anxiety.

Let's say we listen to our friends. Don't buy the Memento Mori Clock. Don't plug it in next to the bed. The trouble is - at least in the semi-affluent portions of post-industrial Western culture-we've already by and large bought the idea of this clock. The main issue is that the idea that time-especially the time of anticipation, the time of the future-has effectively been handed over from human perceivers to networked digital infrastructures understood not merely as technology but rather as "the living mediation of what organizes life."5 Anticipation today is no longer the work of human beings but rather it is the province of technical processes. These processes largely operate beyond human experience even as they have become normalized and ordinary. Following Anaïs Nony, we might say that the Memento Mori Clock substitutes an operative logic of preemption for one of anticipation. Nony writes, "Whereas anticipating is caring for what could come next, preemption is the implementation of one single possibility in the present, and the simultaneous reduction of a virtual and potential future to a single line of interpretation." ${ }^{6}$ Digital technics 
transform the very nature of the future by substituting a machinic logic of preemption for a human experience of care. It is difficult to argue with her. Since World War II technology has been conceived as taking over the human job of anticipation. ${ }^{7}$ Norbert Wiener's insight that machines might more effectively anticipate the flight patterns of enemy aircraft effectively gave rise to cybernetics, the science of communication and control so vital to the development of digital technologies. ${ }^{8}$ More recently, media theorists such as Brian Massumi, Richard Grusin, and Mark B. N. Hansen have all reckoned with the inheritance of this idea in the context of the pervasive media environments of the twenty-first century. ${ }^{9}$ While these accounts focus on the political and philosophical dimensions of technological futurity, I want to emphasize the ordinariness of what Nony calls digital preemption. From Google's autofill feature and predictive text in text messaging applications to machine learning approaches to online discussion moderation and Big Data-driven approaches to marketing (predictive analytics) and law enforcement (predictive policing), a variety of digital technological applications and approaches from everyday life demonstrate how widespread this tendency has become. ${ }^{10}$

Over the last few decades the digital infrastructure of contemporary lived experience has outsourced the human work of anticipation and care. During the same period anxiety has arisen as the paradigmatic mental health issue of the age. In the United States of America alone, the National Institute of Mental Health estimates 40 million adults_or 18 percent-over 18 years of age suffer from an anxiety disorder. ${ }^{11}$ This number is alarmingly high, and it doesn't even account for undiagnosed, undiagnosable, or just more common or low-level forms of anxiety pervasive in post-industrial cultures. As many have noted, anxiety runs rampant in contemporary culture and has a marked if still-poorly understood relation to always-on computing. ${ }^{12}$

While I do not mean to offer a comprehensive explanation for this state of affairs, I believe there is a deep connection between the technological outsourcing of anticipation and the rise of anxiety. Following Ernst Bloch as well as the DSM-V, anxiety is fundamentally a flawed relation to the future; anxiety is anticipation gone wrong. ${ }^{13}$ Following Sigmund Freud's classic distinction, fear has something to fear but anxiety has no object. Anxiety has nothing toward which to put itself in relation or to orient itself. One overwhelming problem with our relation to the future today is that we have increasingly outsourced futurity to digital machines. In Nony's words a society of preemption has replaced one of anticipation. Yet this does not mean that can stop anticipating or seeking a relation to the future. The objects orienting our relation to the future have, however, by and large 
vanished into the opacity of technical processes subtending always-on culture. Our relation to the future has fewer and fewer perceptible objects. It is no wonder then that, as the IPC succinctly states, "we are all very anxious!"

In order to specify anxiety as well as the condition of anxiety in the milieu of always-on computing, consider what Martin Heidegger calls the nothing and nowhere of anxiety. For Heidegger, like Freud, anxiety has no object. One fears something. But one is anxious in relation to a sort of nothing or nowhere. To recall the Heideggerian tone of Nony's argument, anticipation involves "caring." In Heidegger's phenomenological imagination, care [sorgen] captures the fundamental sense in which one orients oneself in relation to the world, e.g. in intending, attending, taking up, seeing to, etc. The challenge of anxiety is that it frustrates care as orientation or comportment. Heidegger writes,

Accordingly, when something threatening brings itself close, anxiety does not "see" any definite "here" or "yonder" from which it comes. That in the face of which one has anxiety is characterized by the fact that what threatens is nowhere. ... that which threatens cannot bring itself close from a definite direction within what is close by; it is already "there," and yet nowhere; it is so close that it is oppressive and stifles one's breath, and yet it is nowhere.

In that in the face of which one has anxiety, the 'It is nothing and nowhere' become manifest. ${ }^{14}$

For Heidegger, anxiety seems paradoxically proximate while yet threatening from a distance. Anxiety has no precise location or even any embodied specificity. He even puts quotes over the verb "see" to indicate how much anxiety evades any specifically modal perceptual apprehension. Still, as Heidegger emphasizes, the nowhere of anxiety takes effect in the mysterious interface of body and world. It "stifles one's breath, and yet it is nowhere." It is difficult to imagine a better description of the anxiogenic effects of the internet!

It may seem paradoxical that a technological milieu so seemingly "ready to hand" as always-on computing might generate so much anxiety, which I'm specifying here an ambient frustration of care. Yet while devices such as smartphones, wearables, and laptops may appear readily, the very thing that defines their power-the network - retains its status as a kind of nothing or nowhere. To be sure, scholars may direct our attention to undersea cables, server farms, or other undeniably material forms of evidence for the network's existence as technological infrastructure. ${ }^{15}$ All the same, the 
network remains something one simply can't point at with satisfaction. As I have written elsewhere, the network exists experientially as a "felt relation of non-relation," a sort of amodal pressure we feel in various ways from phantom cell phone vibrations to the agony of the typing awareness indicator and the progress bar. ${ }^{16}$

Always-on computing has no single experiential correlate, but anxiety is certainly one of its most profound. ${ }^{17}$ The prominence of anxiety as a sort of constantly felt "nothing" or "nowhere" certainly has much to do with the experientially opaque nature of the infrastructure supporting always-on computing in lived experience. However, the trouble is that we know very well that this amodal "nothing" is constantly doing things out of sight that nonetheless impact our lives. Privacy worries typically rule discussions of this sort of below-the-radar-of-the-user activity in the form of NSA dataveillance and data tracking by third-party software on smartphones. These out-of-sight phenomena include the activity of algorithmic forces with designs of their own targeting our dividual or data selves, i.e. our smartphone-related behaviors from GPS data to shopping habits. ${ }^{18}$ As many critics note, digital media do not address liberal human subjects, the sense of personhood I have when I think about myself as "me."19 This does not mean, however, that the felt nothing of algorithmic addresses to my data "self" does not have any effect on my liberal self. It certainly does. And the most general result of this state of affairs is a general rise in anxiety against the networked "nothing" and its aggressively opaque operations.

Anxiety names a felt non-relation to some sort of radically overdetermined "nothing." The name for this nothing today is the network. For Heidegger, as for many other philosophers of anxiety, that nothing has do with death. Importantly, death here is not an "object" toward which one feels anxiety but rather what arouses anxiety. Death is instead a placeholder, an avatar, or way of giving a name to something that can't properly be named, a kind of nothing or nowhere whose felt non-presence generates anxiety. It feels only natural, then, that death would figure plainly in all three anxious objects I discuss in this essay. Death also, of course, names a relation to the future. That future relation is both radically overdetermined (everyone dies) but also characteristically indeterminate (nobody knows what death is). To depart gently from the philosophical seriousness of anxiety and death, let us return to the web's curious scrambling of these ideas in the strange feeling of anxious pleasure occasioned by Memento Mori Clock, which may be grasped as symptom of our uncertain relation to living newly overdetermined lives governed by digital technics operating beyond the scope of human experience. 
Memento Mori Clock's humor can't be easily explained. On the one hand, it's funny. It must be. I mean, who takes seriously the ghoulishly over-serious and foreboding declaration that "it's impossible to cheat the countdown"? On the other hand, maybe everyone? From Holbein to Hamlet to Game of Thrones' valar morghulis ("all men must die"), it is difficult to deny the essential truth of memento mori (remember that you must die). All this, however, seems too serious, too existential for an object resembling an alarm clock (and for anything having to do with internet culture for that matter). And yet, it feels newly revealing to note that the internet vernacular in English for expressing laughter is "I'm dying" or simply "dead." I can't take this seriously, can I? Put otherwise, I honestly can't tell if this is a joke, or if the joke is funny. ${ }^{20}$ The fact that I can't quite tell certainly has something to do with the internet and its ambivalent tone, which frequently renders humor ambiguous. ${ }^{21}$ It also may have something to do with comedy's disconcerting ability to produce and dispel anxiety at the same time. ${ }^{22}$ Let's hold onto this impulse to not take things too seriously (even if we need also to take things seriously!).

Memento Mori Clock certainly solicits anxiety but it also, at least briefly and partially, sweeps anxiety away with a laugh. Such anxious ambiguity, in turn, hinges on our relation to larger techno-cultural dynamics exemplified by Memento Mori Clock, namely: the question of our faith in the capacity of digital processes to determine future realities in advance. Will the number displayed reliably count down to my death? I hope not, but also I hope so? My hoping not is, of course, bound up with my desire not to die or even to address such unpleasantness. But I hope so because I realize that as much as I may not like it I actually do have a lot of faith in the power of digital technologies to anticipate the future on my behalf. In some ways, we simply must live our lives according to a certain faith in digital prediction. Policy decisions based on climate change projections are based on just such predictions, for example. In more everyday circumstances, many of us depend on the predictions of traffic patterns calculated by GPS apps like Google Maps or Waze. Yet something feels amiss when we put our faith too blindly in the capacity of machines to anticipate the world for us on the basis of something as impersonal as data-mining - especially when that world comes into focus in something as singular and personal as the moment of my own death. My death will probably not happen at the same time as the timeline calculated by the clock drawing on data from the World Health Organization-right? Although I can reasonably expect these two times to diverge it's unnerving to imagine the time of my estimated demise ticking down. I know one thing — or probably_and I feel something very different. 
I know and laugh ha! At the same time I also feel anxious and laugh haha. At the root, then, the problem provoked by the ambiguous comedic nature of Memento Mori Clock concerns not any single instance of preemption but rather the problem of its massively general character grasped in relation to the singularity of human life. We have become dependent on machine thinking for a number of reasons. One of the consequences of this increasing dependence is that it is becoming more and more jarring to see ourselves and the very human narrative trajectories of our lives mapped onto algorithmic predictions. We're caught in a twenty-first century bind. We can't tell what to think because we can't anticipate. We can't anticipate because we have outsourced anticipation to digital machines. This is where things get anxious. But crucially, it's also where things get weirdly fun.

The very word "anxiety" seems overwhelmingly negative, or even disabling, as in the expression "crippling anxiety." Yet always-on computing, contemporary media by its very nature intermingles anxiety and pleasure. By doing so it may not turn the "I can't" of anxiety into a rousing "I can." All the same it meaningfully dislodges agency from the death-grip of, well, death. The nothing of the network arouses anxiety but crucially it is not merely anxiogenic. Networks also allow for new forms of sharing anxiety, or for understanding anxiety as relational rather than as purely isolating. Because sharing often simply feels good, this dynamic opens the door for re-articulating anxiety as simultaneously social and pleasurable. Memento Mori Clock bears this out. It arouses anxiety in relation to the overdetermining nature of always-on networks, but there's also something about it driving me to share it. Not because I want to spread my experience of anxiety, but because encountering it together feels good. I don't just want to tell friends about it, I want to be there when they realize what it is and how it works. I want to laugh again at this object, something I can't do again by myself. I want to experience it with someone else. I take pleasure in witnessing and partially sharing the building no-no-no! realization I can read in the face of my friend of just how dreadfully anxiety-inducing this terrible clock is.

Here it bears noting that the Memento Mori Clock is no outlier. Its dynamics recall other more widely known internet phenomena. Consider the famous "This is Fine" meme. In that famous six-panel cartoon from 2013 - reduced to two panels in the 2014 meme-a wall-eyed cartoon dog in a bowler hat sits at a table with a cup of coffee in a room that is on fire. ${ }^{23}$ Smiling, certainly aware of his impending demise the dog states, "this is fine." Like Memento Mori Clock, "This is Fine" expresses a sense of anxious fun. The world burns down around the dog, and he just smiles in the face of it all. The meme depicts a paralyzed, dumb, knowing, and also cute version 


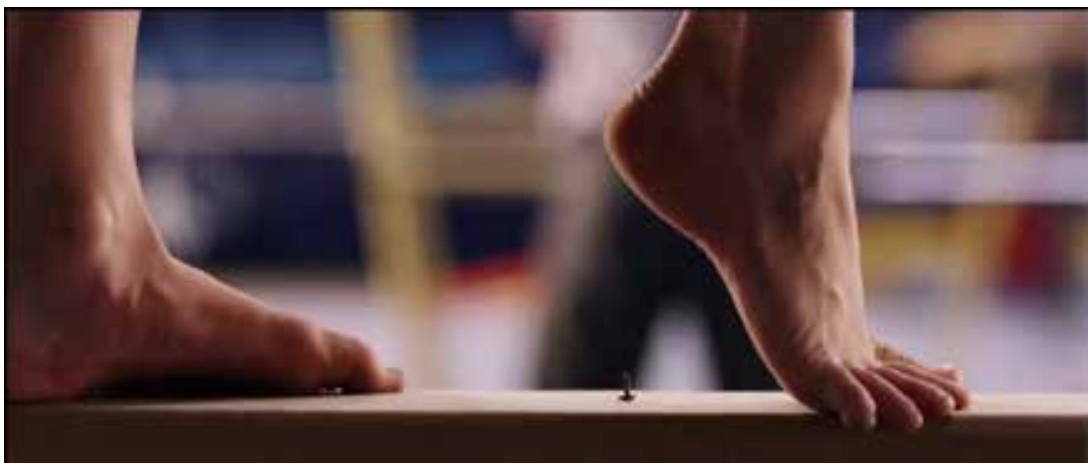

Figure 11.1. Gymnastics scene from Final Destination 5 as seen in the YouTube video I Put Wii Music Over a Final Destination Death Scene.

of contemporary experience today, where the world is often said to be coming to an end or to resemble a dumpster fire. The meme represents the anti-agentive anticipation of disaster in the face of forces outside individual control. It presents an image of anxiety. Yet it also modulates or manages that anxiety through laughter, or the fun of sharing the meme. Memento Mori Clock, then, might be thought of as a more extreme version of "This is Fine." Like "This is Fine," it expresses a sense of anxiety in the context of digital networks. Both thrive on the possibility of constant circulation. And both threaten to act like a declarative statement, the rough equivalent of ending a text message with a period. But circulation keeps alive their complex affective resonances of anxious fun while both continue as a kind of network hot potato because each carries within itself an anxious image of the end of circulation: conflagration and death.

In the last section of this essay I want to discuss one final example: a video entitled I Put Wii Music Over a Final Destination Death Scene. Recommended to me by YouTube's algorithmic anticipation of my viewing pleasures and posted on September 1, 2017 by a Belgian user named MATN, the video currently has over 8 million views about one year later. This three-and-a-halfminute video overlays music from the Nintendo Wii videogame platform's Mii Channel Plaza on top of a clip of a gymnastics routine gone horribly wrong from the 2011 horror film Final Destination 5. Taking a close look at this video will help to elaborate the peculiarly social pleasures of anxiety in the milieu of always-on computing.

The lived experience of always-on computing involves a strangely paradoxical sense of freedom by design. The great achievement of this video's ordinary contribution to social media lies with the ingenious way in which it formally dramatizes the dynamic I've been attempting to elucidate so far: the 
solicitation of anxiety in always-on computing through the combination of an indeterminate posture toward the world (being always on) encountering the radically overdetermining processes and infrastructures governing that make such "indeterminancy" possible in the first place. In this way, the video represents a valuable text for recognizing the pleasurable and social dimensions of anxiety. It does so in its mash-up of light-hearted videogame aesthetics and horror cinema. The combination of music from the Nintendo Wii with footage from Final Destination 5 makes unavoidable the algorithmic nature of death in these movies.

Because this is a clip from a Final Destination movie we know that someone is going to die. The question of who will die, however, is less important than the certain expectation of death. And sure enough, after running through a number of gymnastics exercises - and after the camera teases the viewer with a number of possibilities for how she will die- the gymnast eventually meets a grisly end at the culmination of a Rube Goldberg-esque chain of improbable events (another gymnast on a balance beam steps on a fallen screw leading her to fall off and tip over a container of chalk, which a fan then blows into the face of the main gymnast as she spins on the uneven bars, blinding her during her dismount, and leading her to land so badly that her spine snaps on landing as her body doubles back over her head). In these remarkably cynical movies, Eugenie Brinkema observes, death is not so much a figure as a function of design. Deaths in a Final Destination movie do not add pathos to the psychologization of a character; instead they fulfill a formal contract that might without exaggeration be termed algorithmic. Deaths in a Final Destination are executions in the twin sense of killing and "computational" in the sense of being executable from the point of view of franchise logic. They are also "algorithmic" not merely in the sense of being based in a set of rules reacting to variable conditions. Final Destination deaths are algorithmic also in the sense that they outsource any agential sense of anticipation to the rules themselves as processes governing those same agents and their lifeworld. We don't care about the characters' fates. Because the characters are properly algorithmic we actually can't. Yet because the characters visibly appear to us they help us to grasp better what it is we actually care about.

I wouldn't call the film clip from Final Destination 5 by itself especially anxiety-inducing. If you're watching Final Destination, then you've signed up to enjoy the elaborate execution of its characters. Suspenseful? Yes. Anxiogenic? Probably not. The clip becomes an engine of anxiety, however, when taken out of the context of the movie and overlaid with music from the Wii platform. The light-hearted tone of the repeating melody contrasts 
humorously with the gruesome mock-serious-but-also-somehow-genuinely-serious tone of Final Destination 5. The contrast between the image and the sound tracks makes the clip bearable in a very different way, and also, quite differently enjoyable. In a way, the music opens the possibility for caring in some way about the characters that Final Destination 5 on its own precludes. Instead of just making the clip seem absurd and therefore funny, however, the addition of Wii music in the context of YouTube draws out and makes more literal and unavoidable the networked and "algorithmic" character of Final Destination. As benign as the music sounds, it also evokes the digital genre where one's character almost always dies as a formal function of play. The incorporation of a videogame imagination expands Final Destination's algorithmic aesthetic of overdetermined design by introducing some sense of play in the sense of playability, or "room for play."24 The exhibition of this video on YouTube, moreover, further underscores the video as an ordinary yet paradigmatic object of always-on computing, its status as an aspirational meme, a text ripe for re-mixing and re-circulation. This next "algorithmic" layer of signification underscores and clarifies the social pleasures it affords. But still, what of anxiety?

When I show the video to friends it makes them squirm. I admit this gives me pleasure. Their reported experience of anxiety, however, has very little to do with death. The mashed-up specter of an especially algorithmic form of death merely sets the scene for anxiety. Anxiety's true emergence as a socially pleasurable effect here has everything, however, to do with the question of whether a gymnast will step on the fallen screw lying pointy end up on the balance beam. Importantly, nobody gasps (at least not as much) when the gymnast actually does step on the screw. Yet every time the video features a close up of a heel arched over the screw my friends audibly breathe in through clenched teeth. Two quotes from the top of the comments section of this video seem especially relevant here: "a $\mathrm{n} x$ i e t y" and "why did i only care about the nail that fell." Indeed. Why care about a small puncture wound when certain death is much worse? To follow Heidegger, this makes total sense because one can't actually can't "care" about death. One can, however, very much care about possible pain.

In describing anxiety Heidegger characterizes the failure of comporting oneself toward its "nothing" and "nowhere" as stifled breath. Here I'm guessing he has something like suffocation in mind, especially given the way death arouses anxiety in his philosophical project. The idea of stifled breath recalls for me my friends' reaction to the flaunted possibility that the gymnast might step on the screw. To be clear, their sharp intake of breath is not only the most concrete evidence of the experience of anxiety 
here, it is also both a little in the direction of sympathy but also in the not wholly-contradictory valence of laughter. These reactions may seem like opposites but they each suggest a glitchy frustration in our bodily inability to synch up with or orient oneself in relation to the world. This intake of breath strikes me as important, then, not only for the ways it recognizes anxiety as an ambivalent social mode of aesthetic response generated by this paradigm of ordinary anxiety in the context of always-on computing. It matters that this experience of anxiety takes place, then, not just in reaction to the anticipated possibility of a small puncture in the flesh, but also as a reaction organized in the simultaneously indeterminate and overdetermined thematics of Final Destination 5 and Wii music on YouTube.

Let us say just a bit more about death. Death is justly famous as a figure that arouses anxiety. Try as we might we can't do anything with death. We die; there's not much more to it than that. That futility only reinforces death's association with anxiety. The singularity of death, of my death, further reinforces anxiety as an individualizing, isolating force. The amazing thing about IPut Wii Music is that while the specter of death's algorithmic inevitability arouses anxiety, the felt anticipation of that screw sticking suddenly into a naked foot effectively displaces death and gives a new quasi-formless form of anticipation to anxiety in always-on computing. The imagined ouch! of a screw in a foot gives a new and very different sense of the possible than the specter of death. Namely, we can share this imagined ouch in a way that we can't share the specter of death. The possibility of this event gets to conceptual core of anxiety. Renata Salecl emphasizes precisely the way in which anxiety thrives on the experience of the possibility of possibility. She writes,

When Kierkegaard analysed anxiety, he took it to be something that is linked to possibility in existence. Here anxiety became specifically linked to freedom, or as Kirkegaard says, it is linked to freedom's actuality as the possibility of possibility.... Anxiety is thus linked to the possibility of being able, but, as such, it often appears as a feeling aroused by looking down into a yawning abyss. ${ }^{25}$

As Salecl makes plain, anxiety concerns the possibility of being able. Death-here in the trope of l'appel du vide - arouses anxiety because it feels the grounding of possibility in its utter negation. Possibility wouldn't mean any thing if it couldn't be taken away. Death only arouses the possibility of being able through its negation. By contrast, the screw solicits a vitally distinct sense of anxiety as a sense of embodied ability, what Salecl calls "the 
possibility of being able." Let's not forget that one of the most important and distinctive social capacities of always-on life is the possibility of feeling hurt.

So how much can we actually do? How much are we really able? Are we truly screwed? Being screwed sounds bad. But from a certain point of view it also sounds pleasurable. This is what I Put Wii Music does to us. It tells us, we're screwed. We are all very anxious, and we have plenty of good reasons for feeling this way. From irreversible effects of anthropogenic climate change to terrorism to the resurgence of xenophobic nationalism - haven't you heard? The dark beauty of being screwed, however, is that it can be fun even if it also feels bad. This does not mean we must simply accept our fate by changing our perspective. Being screwed means dealing with it, just as here it has meant recognizing some of the ways we are already doing so. It means not just feeling anxious but also having fun, and having fun means feeling something together. My job here has not been to show that anxiety is somehow socially redemptive, only that that it might be social at all. As a form of ambivalence, being screwed doesn't so much allow us to chart a course for change so much as it helps us to deal with what's hard or seemingly impossible. At the very least, it gives us something to feel and to say: "I'm screwed!" The mere fact of voicing this condition opens up its social character. In the context of always-on computing, as we've seen, the obligation to be communicable makes things a little screwy. Haha. The fun in anxious fun lies with getting worked over together. This may feel like the end but it's where we start today. Nobody gets screwed alone.

\section{Notes}

Thanks to Kris Cohen, Patrick Jagoda, and Kyle Stine for feedback.

1. See, for example, Alex Williams, "Prozac Nation is Now the United States of Xanax," New York Times, https://www.nytimes.com/2017/o6/10/style/anxiety-is-the-new-depression-xanax.html. June 10, 2017.

2. Institute for Precarious Consciousness, "We Are All Very Anxious: Six Theses on Anxiety and Why It is Effectively Preventing Militancy, and One Possible Strategy for Overcoming It," Plan C (April 4, 2014): weareplanc.org.

3. Ernst Bloch, cited by Sianne Ngai, in Ugly Feelings (Cambridge: Harvard University Press, 2005), 209.

4. https://www.mcachicagostore.org/shop/memento-mori-clock-52745.

5. $\quad$ Lauren Berlant, "The Commons: Infrastructures for Troubling Times," Environment and Planning D: Society and Space 34, no. 3 (2016): 393-419, 393.

6. Anaïs Nony, "Anxiety in the Society of Preemption: On Simondon and the Noopolitics of the Milieu," La Deleuziana 6 (2017): 102-110, 103. 
7. Anticipation arguably only persists today in various apocalyptic imaginations from American evangelistic Christianity to the doomsday scenarios of popular fiction across media.

8. See Norbert Wiener, Cybernetics: or Control and Communication in the Animal and Machine, 2nd ed. (Cambridge: MIT Press, 1961); and Peter Galison, "Ontology of the Enemy: Norbert Wiener and Cybernetic Vision," Critical Inquiry 21, no. 1 (Autumn, 1994): 228-266.

9. See Richard Grusin, Premediation: Affect and Mediality After $9 / 11$ (New York: Palgrave Macmillan, 2010); Mark B. N. Hansen, Feed-Forward: On the Future of Twenty-First-Century Media (Chicago: University of Chicago Press, 2015); Brian Massumi, Ontopower: War, Powers, and the State of Perception (Durham: Duke University Press, 2015); and Shane Denson, Discorrelated Images (Durham: Duke University Press, 2020).

10. See Mark Andrejevic, Infoglut: How Too Much Information is Changing the Way We Think and Know (New York: Routledge, 2013).

11. American Psychological Association, "Data on Behavioral Health in the United States," American Psychological Association. www.apa.org.

12. See, for example, Jean Twenge, iGen (New York: Atria Books, 2017).

13. As defined in the DSM-V, "anxiety is anticipation of a future threat." It overlaps with but is distinct from fear: "with fear more often associated with surges of autonomic arousal necessary for fight or flight ... and anxiety more often associated with muscle tension and vigilance in preparation for future danger and cautious or avoidant behaviors." See Diagnostic and Statistical Manual of Mental Disorders: DSM-5 (Arlington, VA: American Psychiatric Publishing, 2013).

14. Martin Heidegger, Being and Time, trans. John Macquarrie and Edward Robinson (New York: Harper Perennial, 1962), 231.

15. For example, see Nicole Starosielski, The Undersea Network (Durham: Duke University Press, 2015).

16. James J. Hodge, "Sociable Media: Phatic Connection in Digital Art," Postmodern Culture 26, no. 1 (September 2016): n.p.

17. Scott C. Richmond, "Vulgar Boredom, or What Andy Warhol Can Teach Us About Candy Crush," Journal of Visual Culture 14:1 (2015): 21-39.

18. See John Cheney-Lippold, We Are Data: Algorithms and the Making of Our Digital Selves (New York: New York University Press, 2017).

19. See Kris Cohen, Never Alone, Except for Now: Art, Networks, Populations (Durham: Duke University Press, 2017).

20. See Damon R. Young, „Ironies of Web 2.0,“ Post45 2, May 2, 2019: https:// post45.org/2019/05/ironies-of-web-2-0.

21. See Whitney Phillips and Ryan M. Milner, The Ambivalent Internet: Mischief, Oddity, and Antagonism Online (Malden, MA: Polity, 2017).

22. Lauren Berlant and Sianne Ngai, "Comedy Has Issues," Critical Inquiry 43, no. 2 (Winter, 2017): 233-249, 233.

23. See "This is Fine," Know Your Meme (2015-), knowyourmeme.com. 
24. See Miriam Bratu Hansen, “Room-for-Play: Benjamin's Gamble with Cinema," October 109 (Summer 2004): 3-45.

25. Renata Salecl, On Anxiety (New York: Routledge, 2004), 53; and Soren Kierkegaard, The Concept of Anxiety, trans. Reidar Thomte (Princeton: Princeton University Press, 1980).

\section{About the Author}

James J. Hodge is Associate Professor in the Department of English and the Alice Kaplan Institute for the Humanities at Northwestern University. He is the author of Sensations of History: Animation and New Media Art (University of Minnesota Press, 2019). He is currently writing a book about the aesthetics of always-on computing. 



\title{
12. Beep: Listening to the Digital Watch
}

\author{
Sumanth Gopinath
}

\begin{abstract}
This essay considers the intertwined histories of the word "beep" and the simple, single-oscillator tone by examining the digital watch of the late 1970s-early 1980s. The watch and its beep marked a key economic and technological development in which the US, as global hegemon and economic powerhouse, was not the dominant agent. They also prefigured the tinkling sound of ringtones on mobile phones and initiated the mass mundanization of digital beeps, now shorn of the symbolic power of recondite, expensive, and classified Cold War-era technologies. The watch beep's hardwired nature allows us to hear connections between that historical moment's different temporal scales, direct linkages between which would soon be imperceptible thanks to the digital economy's ever increasing abstraction.
\end{abstract}

Keywords: beep, digital watch, sound studies, piezoelectricity, world-systems theory, integrated circuit (IC)

Beep. This word denotes several associations in English, including its noun and verb forms, its evocative epizeuxis beep-beep, and its description of a device, the beeper. The Oxford English Dictionary reveals that the beep is a twentieth-century phenomenon, initially indexing the bustling modernity of urban soundscapes and transit and expressed through the obtrusive car horn. ${ }^{1}$ Plosives in the word's beginning (voiced) and ending (unvoiced) frame a close front unrounded vowel that accentuates higher frequency content, particularly in American English. Sounding like the signal it describes, the word's onomatopoetic qualities grab your attention, quickly. The oldest relevant entry in the dictionary is Edmund Wilson's first novel I Thought ofDaisy (1929), as spoken by the titular character, Daisy Coleman, a chorus girl who is the narrator's love interest and who describes car horns thusly:

Volmar, A. and K. Stine (eds.), Media Infrastructures and the Politics of Digital Time: Essays on Hardwired Temporalities. Amsterdam: Amsterdam University Press, 2021 DOI 10.5117/9789463727426_CH12 
“'It's a shout. There's a toot-toot, and a beep-beep - and an oorah-and a blah-blah, and a blurp-blurp. - Dad's was a kind of a oorah - and it was a humdinger, too!"2 The horn's sound is not uniquely captured by "beep," and today a horn is typically described as honking. A search of The New York Times reveals that the word doesn't appear in the paper until the 1930s, when describing a bicycle horn. ${ }^{3}$ In the postwar period, "beep" expanded beyond vehicular horns to designate electronic signals, including those in military technologies, touch-tone dialing, and other telephonic sounds; ${ }^{4}$ experimental electronic music and its uneven migration into pop/rock; science fiction and space travel (partly via the Sputnik beep); ${ }^{5}$ and radio alert and signal-scrambling systems like CONELRAD (Control of Electromagnetic Radiation) and the Emergency Broadcast System (EBS). ${ }^{6,7}$ The expanded use of the word "beep" suggests that beeps became central to the US Cold War soundscape.

But if the initial conjoining of the beep and single-oscillator signal occurred during the period spanning the late nineteenth century and World War II, and a new crystallization began after the war, it would be the long 1970 s that made the beep newly ubiquitous by embedding them into devices large and especially small: appliances, phones, games, computers, and pagers. Moreover, due to the global growth of Japanese electronics production and sales, the story was no longer predominantly a US-centric one. Making that technological and sociocultural transformation possible was the rise of semiconductor electronics, which enabled smaller and smaller components to be tucked away silently into ever more ubiquitous devices. As Ross Bassett puts it, the semiconductor transistor "is a base technology of late-twentieth-century and early-twenty-first-century America. Through it digital electronics have entered almost every area of American life, first through the calculator, then through the digital watch, and finally through the microprocessor." 8 Despite their pervasiveness in America and worldwide, microelectronics are unseen and unheard, known only through their effects. It is fitting that they have been accompanied by equally ubiquitous, beeping small speakers. ${ }^{9}$

The widespread mundanization of the smartphone and hence portable personal computer, with its sophisticated audio generation and playback capabilities, have seemingly propelled the world into a newly intensified dependence upon and engagement with integrated circuits (ICs), microprocessors, and the complex sounds they enable. But we still live in the era of the beep, which is unlikely to disappear anytime soon, given its utility and simplicity. If the beep acts as a deep social layer composed of myriad hardwired temporalities that continues to either interact with or ignore 
sound reproduction, ${ }^{10}$ its formation merits further investigation. A crucial development in the beep era is that of digital watch, in which the IC met the beep in a mobile, mass-market context for the first time.

The history of the digital watch is complex and dramatic. It involves the belated application of the nineteenth-century discovery and theorization of piezoelectricity, international economic battles between the established Swiss mechanical watch industry and newer, upstart watch industries based in the United States and Japan, ethico-moral debates over the importance of learning to read the analog clock face, ultimately leading to its coexistence with the numerical watch display, and the inauguration, along with the calculator, of a series of consumer fads for digital devices beginning in the 1970s. In what follows, I consider the digital watch at multiple temporal scales. First, I recount the long history of the watch industry's transnational political economy, including its transition to digital timekeeping. The industry's temporality is interpreted here at the macro-temporal level of Arrighi's "long century," in ways that dovetail with the century-long, US-hegemonic history of the "beep" word and concept. ${ }^{11}$ I then explore the micro-temporality of the digital watch, as produced by the piezoelectricity of quartz crystals and microprocessor-calculated digital timekeeping. I continue by examining the digital watch's sounds and end by noticing their presence in social spaces; these sounds seem to exist at a widely meso-temporal scale from minutes to months and years. The patterned alignment of these particular components and their corresponding social practices can be interpreted as a form of hardwired temporality, but the simplicity of the micro-temporal components in early mass digital timekeeping allowed a direct and perceptible connection via the meso-temporal. To anticipate my conclusion, listening to the digital watch allows us to further connect these temporal scales, by articulating a beep (and its means of generation) with the incipient decline of US economic hegemony. By becoming quotidian, the beep-which prefigured the more versatile and abstracted mobile phone and its sounds-lost its valences of power and potential.

\section{The Political Economy of the Watch Industry}

The digital watch upended the traditional global hierarchy of the watch industry, leading to the divide between luxury and economy exports stemming primarily from Switzerland and East Asia, respectively. Before the digital watch's emergence, the industry was dominated by Swiss watch firms, as it had been since the mid-nineteenth century. ${ }^{12}$ That dominance was arguably 
a hard-won product of economic and technological contestation in Europe over the course of the early modern period. Several contributing factors include the emigration of French Hugenot watchmakers to Switzerland in the sixteenth century, which brought crucial new talent to the country and arguably crippled French watchmaking; the peculiar form of decentralized social conservatism characteristic of Swiss industries, leading to the gradual adoption of institutional changes and greater inclination toward honing existing practices; and its comparatively high ratio of watchmakers to parts manufacturers, encouraging the creation of a greater variety of products in different price ranges. ${ }^{13}$ These developments led watchmakers in Geneva to become increasingly competitive with the more established British watchmaking industry in the eighteenth century. Then, with the rise of yet another Swiss region for watch production in the Jura mountain valley region, centered in Neuchâtel, in combination with generally lower wages in Switzerland, the country's products began to undercut British sellers at the end of the eighteenth century and into the nineteenth century. Long being an emulative industry up to the mid-eighteenth century, following innovations from Britain or France, Swiss watchmakers increasingly took the lead in new development, creating standardized tests, competitions, and watchmaking schools, all with the strong support of the state and contributing to Swiss preeminence in watch production by the mid-nineteenth century.

The primary contender for industrial dominance in the mid- to late nineteenth century was the US. Driven by the demands of railroad scheduling, the US watch market was temporarily closed to exports due to wartime protectionism during the Civil War, giving a boost to postbellum industrial growth in US watch manufacturing, which became increasingly rationalized. US firms soon became strongly competitive with Swiss makers, who were slow to adopt factory production methods. Industrialized US watch firms, such as the Boston-based Waltham Watch Company, were of crucial importance in establishing the quality of US watches, and the Elgin Watch Company, located near Chicago, in turn drew on the expertise (and sometimes personnel) of Waltham, leading to a powerful, two-company trust at the forefront of the US watch industry in the late nineteenth century; the Hamilton Watch Company, based in Lancaster, Pennsylvania, was another important late entrant into the market that would assume an ever stronger role among US firms. And yet, in $1885^{-1905}$, the Swiss watch industry upgraded itself partly on the American model, reducing small-scale production and moving toward vertical integration, and by 1910 Swiss watches again dominated the global market. Crucial was the rise of the wristwatch (instead of the older pocket watch), which Swiss makers produced more successfully 
than US firms, who were hampered by "a uniform regulatory regime for the production of railroad watches using machine-manufactured parts." ${ }^{14}$ Swiss watch industry dominance continued through the interwar and early postwar periods, with market share for low-end watches only facing significant competition from US-based Timex in the 1950s and 196os.

The rise of the Japanese watch in the 1960 s and after was completely unexpected by the Swiss and American watch industries. Although Japan industrialized in the nineteenth century and sustained a sizable watch industry led by Seiko, Citizen, and Ricoh in the early twentieth century, its greatest advances in the watch industry occurred during the renewed industrialization of the postwar period, after the devastating WWII bombing of its factories and productive forces. Its credentials were boosted by a key development. As Amy Glasmeier notes, "When Seiko was selected as the timekeeper for the 1964 Winter Olympics, held in Sapporo, Japan, its reputation was greatly enhanced. In full view of the entire world, the Japanese watch company demonstrated what had heretofore been the exclusive purview of the Swiss: to flawlessly keep, record, and display time at exceptionally high levels of accuracy."15 But the critical commodity for the Japanese watch industry was the digital watch, which ultimately led Japan to become the world's largest watch producer, with annual production figures growing from nearly 700,000 to over 86,000,000 watches from $195^{\circ}$ to $1980 .{ }^{16}$ US-American and Japanese scientists and companies collaborated and competed in producing high-quality ICs (and later liquid crystal displays or LCDs), first used in calculators and then watches: when they entered some aspect of this market at all, US electronics firms often gave up on or mistimed their investments in these technologies, to the benefit of Japanese producers. ${ }^{17}$

Despite the relative weakness of the US watch industry after WWII, it was competition between American and Japanese firms that would prod the development of the digital watch, with the latter ultimately winning out. After creating initial prototypes, Swiss firms stayed on the sidelines to their detriment - until Swatch, founded in 1983, helped put an end to the "quartz crisis" of that country's watchmaking industry. ${ }^{18}$ In retrospect, the earlier rise of American timekeeping - in contrast to the longer-established British and Swiss industries - signaled the corresponding rise of US-American hegemony in surprisingly direct ways (with the British watch industry's supersession and Swiss industry's development arguably linking not only to the early-modern-historical lineage mentioned above but also to the decline of British hegemony and to central-European bids for global dominance in the mid- to late nineteenth and early twentieth centuries, respectively). 
This narrative suggests that the timekeeping business was fundamental to the capitalist world-system more broadly-which might be expected, given the essential role of clocks in the management of modern markets, transportation, labor, and state administration. The Japanese development of the digital watch likewise signaled a new possible capitalist hegemony in East Asia-later furthered by China's emergence as "the workshop of the world." ${ }^{19}$ Bound up with the long century of American hegemony, the beep was absorbed and expanded dramatically in Japan and East Asia, alongside digital devices more generally. ${ }^{20}$

\section{Piezoelectricity and the Quartz Watch}

The foundation of quartz timekeeping is the phenomenon of piezoelectricity. Discovered by Jacques and Pierre Curie in 1880, it involves the generation of an electrical polarization by applying mechanical stress to certain materials, especially crystals, metals, and ceramics. This polarization creates the possibility of generating an electrical current from mechanical pressure to a piezoelectric material, but the reverse effect - generating a mechanical movement from an electrical charge — was also predicted by Gabriel Lippmann one year after the Curies' discovery. Although the basic mathematical theorization of piezoelectricity was completed by Woldemar Voigt in 1890, it was first employed in devices during WWI, when early efforts to detect submarines via ultrasonic waves (later termed SONAR by the US military) made use of quartz-based transducers. In 1921 Walter Cady created the first quartz crystal oscillator, and in 1927 scientists at Bell Labs produced the first quartz clock, using quartz as a stable oscillator. ${ }^{21}$

With the emergence of the integrated circuit in the 1960 , quartz clocks came into widespread use; their basic principle can be understood as follows (fig. 12.1). First, a battery (b) powers a transistor oscillator (not shown) that activates the crystal resonator $(a)$, causing it to vibrate and hence transform electrical energy into elastic energy. The resonator smooths out the irregularities of the oscillator, thanks to the very low power loss and high stability of the quartz crystal. The resonator then reconverts the vibrations into a highly stable electric current, sending it to the microprocessor $(d)$, which counts the number of pulses from the quartz (which is conventionally designed to oscillate at 32,768 or $2^{15} \mathrm{~Hz}$ ). The microprocessor then repeatedly divides the pulses by 2 in order to reach $1 \mathrm{~Hz}$ (or 1 cycle/second) for the purpose of keeping time. The output information is then sent to the digital display $(e)$ or, if it is an "analog" quartz clock, to a watch movement mechanism that 


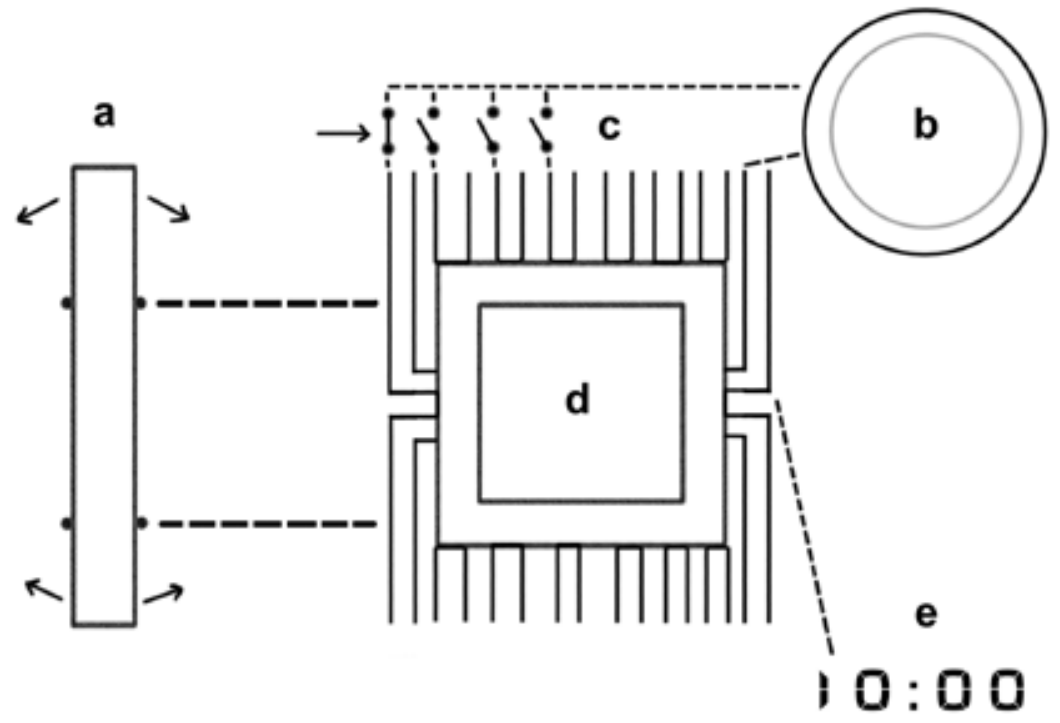

Figure 12.1. Diagram of the quartz wristwatch structure, after David Penney, in David Landes, Revolution in Time: Clocks and the Making of the Modern World (London: Viking, 2000), p. 376.

moves the second, minute, and hour hands of the clock face. (Hence, we appreciate that just about all watches today, irrespective of their display method, use digital timekeeping circuitry - even though "analog" quartz clocks are typically described as "electronic" rather than "digital." Mechanical watches, presently a tiny minority of watches worldwide, are identified by smooth, rather than pulsed, second-hand movement.) In a digital display, a number of switches $(c)$ are also used to control other, ancillary functions like the alarm, stopwatch, and light, and thus are also mediated by the microprocessor. ${ }^{22}$

In concerning the sonic aspects of the digital watch, we must further examine and contextualize the series of switches $(c)$ that drive the watch's ancillary functions. The earliest digital watches were sold as luxury goods, in part as a result of the initial high cost of the timing circuits, and generally competed in the high-end watch market. The first electronic ("analog") quartz watch on the market, the Astron SQ made by the Japanese maker Seiko, was released in 1969 for $45^{\circ}, 000$ yen (or $\$ 1,25^{\circ}$ US), on the heels of several successful prototypes produced in Switzerland at the Centre Electronique Horloger (CEH) for competition in the Neuchâtel time trials in 1967. The American maker Hamilton, abandoning its unsuccessful efforts to produce an analog quartz watch, sought instead to make a fully digital watch, with a red light emitting diode or LED-based numerical readout 
partly inspired by Hamilton's digital timepiece for the film 2001: A Space Odyssey (1968). ${ }^{23}$ Featured in the James Bond film Live and Let Die (1973), the Pulsar cost \$2,100 upon first release in $1972 .{ }^{24}$ However, the price of microelectronics components soon dropped precipitously, inaugurating a series of price wars within the industry. As Amy Glasmeier notes,

From 1974 to 1975 , the price of a digital watch dropped from $\$ 125$ to $\$ 50$. The next big step downward was forecast for 1977 : some industry watchers believed that prices would fall to $\$ 20$. Then Texas instruments stunned the market by introducing a plastic-encased digital watch for $\$ 9.95$ ! The actions of Texas Instruments were quickly followed by other semiconductor makers who hoped to destabilize and thwart further actions by watch assemblers who could not buy components cheaply enough to compete at the $\$ 20$ level ... once Timex's exclusive domain. ${ }^{25}$

The increasing overcapacity of the semiconductor industry signaled the end of the integrated circuit's "market-pull" phase, in which demand for new semiconductors clearly outstripped production rates, to a new, "technology-push" phase in which semiconductor manufacturers pushed their way into new industries like watchmaking and undersold established firms to meet the demands of expanding fixed capital sunk in production equipment and facilities. ${ }^{26} \mathrm{~A}$ new, mass-oriented, spectacularized sales ethic soon replaced the older, luxury-goods oriented marketing strategy, as seen in a comparison between a mid-1970s watch ad by the Swiss-British luxury brand Rotary (featuring an older, less efficient red LED display) and a 1978 Seiko Memory Bank Calendar Watch commercial (featuring the then-newer LCD, science-fictional synthesizer music, and a tech-functional ethos). ${ }^{27}$

The micro-temporality of the digital watch was bound up with new economic realities within the industry: it was cheaper and more accurate, and its digital temporalities bypassed the previous mechanical temporalities of the traditional watch movement. The digital watch's temporality had new visual indicators, chiefly the display that read out digits instead of hands on a dial, but also its employment of a microprocessor and speaker system that typically resounded its inner workings through a beep.

\section{The Sounds of the Digital Watch}

Within an increasingly competitive sales environment, companies in the mid- to late-197os began producing multifunctional watches, including 
calculator and calendar watches, and incorporated sound into them. Some early digital sound watches were alarm watches, and the alarm soon became standard on digital watches, which often emitted short beeps when a button was pressed. ${ }^{28}$ Among the earlier examples included the 1977 Seiko Ao39 Quartz LC Alarm Watch, which featured two different alarms with two volume settings, an hourly signal, and a stopwatch function (likely including start and stop beeps). By the turn of the new decade, novelty sonic watches emerged, including some that played several different melodies, offered video games, and even talked in the case of the OMNI Voicemaster. According to one news article, this latter product "looks like a normal digital watch but, when a small button next to the face is pushed, a male voice announces the time-hour, minutes and a.m. or p.m. The timepiece also has a 24-hour alarm - with the tune of Boccherini's Minuet. When the set time arrives, the watch states the time, then plays a melody for about 20 seconds. Unless the wearer resets the alarm control within five minutes, the watch announces 'Attention please,' repeats the time and urges 'Please hurry.' The watch can also be used as a talking stopwatch." ${ }^{29}$

Perhaps the most important of these watches in terms of sales and musical content were the Casio Melody Alarm watches first appearing at trade shows in 1980 and released on the market the following year. ${ }^{30}$ These watches featured twelve melodies, divided into two groups. The first group, consistent across most of the watches, provided alarms on the hour or as coordinated with the watch's calendar function, such as playing "Happy Birthday" on a designated birthday, "Jingle Bells" on Christmas Day, the "Big Ben" chime for hourly alarms, and Mendelssohn's "Wedding March" for a wedding or anniversary. The second group involved daily alarms that varied more frequently according to watch model; in each model, however, the alarm was one of seven melodies coordinated with the days of the week. The variety can be seen across two production batches of the Casio Hio4 Melody Alarm Watch with different pre-programmed melody modules (table 12.1). Module 82 features a weekly schedule beginning with F. W. Meacham's 1885 march "American Patrol" on Monday and a number of European "folk songs" from Tuesday to Friday, before ending on the weekend with the Japanese song "Sakura Sakura" on Saturday and the main tune of Schubert's Moment Musical No. 3 on Sunday. The melody collection here seemingly trumpets Japan's recent inclusion into the global north and group of politically powerful nations, comprising the US and most of the dominant European countries (France, Russia, Italy, Spain), with Sunday being reserved for an Austrian classical composer. ${ }^{31}$ Module 142 , in contrast, is more scattershot and less inclusive internationally. 
Table 12.1. Casio H104 melody watch instructions, listing of melodies for two separate sound-production "modules"

\begin{tabular}{ll} 
& \multicolumn{1}{c}{ (Module No. 82) } \\
\hline $\begin{array}{l}\text { Day of week } \\
\text { Monday }\end{array}$ & Melody \\
Tuesday & American Patrol (F. W. Meacham) \\
Wednesday & Santa Lucia (Napoles folk song) \\
Thursday & Marche Royale (French folk song) \\
Friday & Kalinka (Russian folk song) \\
Saturday & Sakura Sakura (Japanese folk song) \\
Sunday & Moments musicaux (Franz P. Schubert) \\
\hline & \\
\hline $\begin{array}{l}\text { Day of week } \\
\text { Sunday }\end{array}$ & Melody \\
Monday & Ungarische Tanz, Nr.5, F moll (Brahms) \\
Tuesday & "Carmen." Votre toast, je peux vous le rendre (G. Bizet) \\
Wednesday & Little brown jug (American folk song) \\
Thursday & L'amour est bleu (Andre Popp) \\
Friday & A walk in the Black Forest (Horst Jankowski) \\
Saturday & Yellow Rose of Texas (American folk song) \\
\hline
\end{tabular}

It is weighted more heavily toward classical music (Brahms, Bizet, and Mozart), with some Europop (André Popp), European easy listening (Horst Jankowski), and a couple of nineteenth-century US minstrel tunes described, again, as "folk songs" ("Little Brown Jug" and "Yellow Rose of Texas").

With the exception of Popp, Jankowski, and "Happy Birthday," these melodies were no longer protected by copyright and could be adapted freely. However, at least one version of the watch (perhaps the M-1230) reproduced mostly English-language popular songs from the 1960s and 1970s, including "Never on Sunday" (a Greek and then English-language hit song from the same-titled 1960 film), Simon and Garfunkel's "The Sound of Silence" and "Scarborough Fair (Parsley, Sage, Rosemary and Thyme)," the Beatles' "Ob-La-Di, Ob-La-Da," "Rhythm of the Rain" (a 1962 hit for the Cascades), and Billy Joel's "The Stranger" (1977). These copyrighted tunes were likely used without proper licensing or royalty payments. (This was well before the US/UK music publishing industry had become extremely driven to secure intellectual property rents in all possible venues, and Japanese corporations' geographical, linguistic, and legal distance surely hampered enforcement.) 
Finally, some Casio Melody Alarm watches included an LCD scale-display showing notes corresponding to each melody's pitches-specifically, their white-key equivalents or counterparts represented within a C-diatonic gamut, like a visualization of fixed-do solfège. Casio and other companies eagerly demonstrated the utility of LCDs: some of their watches utilized an LCD dial imitating the analog clock face, while others used the display for portable video games.

By the 1980 s, sounds on these watches were usually made by piezoelectric speakers, which were developed over the course of the 1970s. Using the same effect as the quartz timekeeping mechanism, a piezoelectric speaker is a small transducer that transforms electrical signals into mechanical energy without the mediation of a wire coil or magnet (as with standard speakers). Scott Chou of Hattori Seiko Co. (Seiko's name in 1983-1990), noted in 1989 that in most watches the piezoelectric element is a type of ceramic foam connected to a thin silver contact, resonating at a frequency of about 4,000 $\mathrm{Hz}$ and taking advantage of the human ear's great sensitivity at 4,000-6,000 $\mathrm{Hz} \cdot .^{32}$ The benefits of the piezo speaker were multiple. It required low power, a primary concern during the 1970 (the red LED display fell out of favor partly due to power needs). Like the watch mechanism itself it was highly reliable. And, most importantly, it was remarkably compact - taking up almost no space within the watch itself (present-day digital watches nestle it snugly between the microprocessor and the battery).

Chou's description of the alarm's frequency seems correct: the standard digital watch beep is set at $4,096 \mathrm{~Hz}$, the oscillating frequency of the piezoelectric quartz crystal, $32,768 \mathrm{~Hz}$, divided by $2^{3}$ or undergoing a half division three times. The watch alarm uses the original oscillating frequency as the quartz watch timekeeping system to power the alarm, almost certainly using the same microprocessor to compute the alarm's frequency. The pitch is a very high $\mathrm{B} 7$ or flat $\mathrm{C} 8$ (about 38 cents flat). Other digital alarms and signals use the same principle, sounding at the same frequency or an octave or two below-2,048 Hz (or high $\mathrm{B} 6$, flat $\mathrm{C}_{7}$ ) or 1,024 $\mathrm{Hz}$ (or high $\mathrm{B}_{5}$, flat $\mathrm{C6}$ ). In the earliest watches, cost was likely a determining factor in the selection of the alarm frequency; the alarm had to be solidly within hearing range, and $8,912 \mathrm{~Hz}\left(32,768 \mathrm{~Hz}\right.$ divided by $\left.2^{2}\right)$ is a little bit high to be effective as an alarm (and much less audible for elderly hearing people ${ }^{33}$ ). The highest possible frequency would entail the smallest number of half-divisions (which would not necessarily mean a cheaper circuit - that would depend on the most efficient Boolean logical reduction of the alarm clock circuit). Given the frequency's commonness, it is possible that 4,096 $\mathrm{Hz}$ requires the smallest (and likely cheapest) piezoelectric sounder needed 
to produce the sound. Moreover, since low frequencies generally require more power to be heard and given this frequency's placement within the maximally perceptible frequency range of hearing, it probably uses the least battery power. ${ }^{34}$

\section{The Social Fallout of the (Sounding) Digital Watch}

If the macro-temporality of the digital watch industry is its long centuries and the micro-temporality of the digital watch is its quartz crystal vibrations, it is the meso-temporality of the watch's use, including its beeps, that has shaped everyday life. Minutes, hours, and days structure that use, through its orientation toward the working day; the watch's alarms enact both the production of labor discipline (such as daily waking for work) and reproductive leisure (such as the late 1970s and early 1980s running boom, to which its stopwatch function became fundamentally tied). ${ }^{35}$ Weeks, months, and even years were marked by additional uses, from the calendrical functions identifying the first two to the longer periodicities of leap-year date corrections or replacing the watch's battery, acts all accompanied by ever-present beeps.

For a time, the digital watch seemed to be everywhere. Indeed, during the 1980 s, a considerable ressentiment against it gathered steam, with much of the energy trained upon the digital time display. As James Sterba wrote in 1982,

The anti-digit traditionalists have staged a comeback in the last few years, partly by stealing a word from the computer business - analog - to space-age the name of their old standard bearer. It is no longer just a watch. It is an analog watch, which means it represents one quantity - time - by another quantity, the motion of its hands on a dial. They argued that there might be hidden danger to children, growing up on digits, who could read time by simply knowing numbers but could not discern it analogously from a watch or a clock with hands. ${ }^{36}$

Aiding the antagonists were the perceived sonic failings of the digital watch; as the watch historian and partisan for the old mechanical watch movement David Landes argued, "the tick of a good timepiece is a delight to those who can appreciate a strong, regular beat," whereas the British designer Richard Porch complained that the digital watch is "a silent affair that requires no attention." 37 Except, of course, when it beeps-especially as it often does on the hour, every hour, with a quasi-humanizing or 
science-fictional chime likely intended to give some sonic character to the otherwise silent device. Indeed, the collective annoyance with the digital watch came to the fore in public art and performance spaces, generating a mild "digital watch rage" recalled two decades later by ringtone rage in similar circumstances. One journalist connected such experiences to broader frustrations with the beep:

The contemptuous little "beep" never comes at the crescendo of a Wagner symphony. It happens only during a dramatic pause in Hamlet's soliloquy, the turning point in a critical sales presentation, or at the perfectly inappropriate moment of a torrid love scene. In the worst case, a sudden, uncontrollable beep has the same social impact as a sudden, uncontrollable belch. If digital watches were the only devices openly mocking their owners, life in the Technobabble Jungle wouldn't be so frustrating. ${ }^{38}$

But a major difference between more recent cellphone interruptions and digital watch intrusions is the regularity (and predictability) of the routine. One editorial writer lamented,

To be in a movie theater now as the hour changes is to hear a beep chorus. It sounds as though half the audience is wearing those digital watches that emit one beep on the half-hour and a vigorous beep-beep on the hour. The chorus would be tolerable if everybody's watch chimed in unison. But the movie theater beep-beeps start around 8:58:30, gather in a crescendo, and then sputter along until 9:02 or so-a longish distraction, and a puzzling one. ${ }^{39}$

The opponents of the fully digital watch and its beeps won out, relegating the device to second-fiddle status within the industry and restoring the analog dial to its once-primary position..$^{40} \mathrm{But}$, as historians Carlene Stephens and Maggie Dennis put it, "Behind the dial of most new watches, though, hummed an electronic heart. ${ }^{\prime 41}$ Indeed, by 1997, mechanical watches accounted for only 7.9 percent of total volume and 44 percent of sales value globally. But the beeping digital watch had the last laugh: the same basic principle of sound production made its way into the mobile phone, providing the technological basis for the extremely annoying monophonic ringtone during the 1990s and early 20oos. Synchronized to cellular networks, the beeping phone became the new digital watch; in doing so, it bypassed the problem of accurate, device-based timekeeping altogether. 


\section{Conclusion}

Despite the appearance of critically praised, wrist-based devices such as the Pebble Watch, Samsung's Galaxy Gear, and the Sony SmartWatch and the popular self-generated-data-tracking device FitBit, no device has yet inspired a sufficient degree of excitement to propel wrist-based wearable devices into the leading edge of popular technology consumption in the new millennium. The closest exception might be the Apple Watch, first released in 2015. ICT industry boosters hoped that the device would do for the smart watch what the iPhone did for the smartphone and the iPad for the tablet computer, and although its popularity is steadily rising, it is difficult to predict its future..$^{42}$ It initially targeted tech enthusiasts, fitness watch users, Quantified Self movement participants, wealthy consumers, and those nostalgic for outdated science-fiction protagonists, including Knight Rider's Michael Knight, Inspector Gadget's niece Penny, and Dick Tracy. For now the Apple Watch is still most functional when tethered to an iPhone. ${ }^{43}$ But it can make the same preset ring and alert sounds as the iPhone, and, like many phones today, these functions are frequently silenced.

The smartwatch commodity could help to close the gap created by the mobile telephone, which in the early to mid-2ooos caused a precipitous drop in wristwatch sales among younger consumers especially. ${ }^{44}$ The conflict between watch and phone- two technologies that offer what Landes calls "cheap time"45 — masks how they have influenced one another, particularly in the era of the integrated circuit, which permits a degree of repurposing remediation hitherto unimaginable. The digital watch, an early wearable digital technology, was a precursor to the modern mobile phone, particularly in the way it accumulated features, including some of the same sonic ones. By listening to the digital watch, we learn something about not only the relationship between two world-historical devices but also the corresponding states of the capitalist world-system that made their emergence possible.

The parallels between the respective sounds of the digital watch and the mobile phone are easy to draw. ${ }^{46}$ The digital watch's beep became the simple digital ringer of the mobile phone, and the melodic watch became the monophonic ringtone. Ringtone rage in the public sphere and at performances in the early 2000 s was prefigured by the public disturbances of the half-hourly chimes of the digital watch two decades earlier. Both the digital watch and mobile phone used their sound production systems to incorporate timers and handheld video games, and the digitized voices of novelty watches even anticipated the voice assistants of Siri, Cortana, 
and Alexa. And, the global political economies of both devices revealed a tripolar dynamic, with competition between specialized locales in Europe (Switzerland and Nokia's Finland) and Japan and the US being relatively shut out of the process while previously contributing technologies that were transferred outward (integrated circuits and cellular telephony, single-oscillator tone-generators and FM synthesis). Moreover, the digital watch's trebly, tinkly beep presaged the monophonic ringtone's aesthetics, which are arguably a sonic corollary of global-regional cute (kawaii) culture-a culture that articulates unevenly with Japanese state-economic power and now exists worldwide, including in the US. ${ }^{47}$ But the differences in device purpose and design and the historical contexts of technological development reveal clear dissimilarities: with the digital watch, there was no progression in sonic fidelity, exhibited by the shift from monophonic, to polyphonic, and to sound file as with the ringtone (and numerous precursor technologies); nor did the digital watch make its tunes programmable or uploadable, leading to a lucrative para-industry comparable to the ringtone industry. And the flexibility of assigning distinct sounds to different contacts, functions, and apps in the cellphone (and smartphone and smartwatch) bespeaks a world of distributed, individualized labor-time management exploited by the digital gig economy and only hinted at by older multifunctional digital watches. ${ }^{48}$ Indeed, it is the very rigidity of the simple digital watch's beep-unlike the flexibility of and rapid changes in the mobile phone's sonic production - that allows one the rare possibility of hearing the micro-temporality of the digital watch. It is the watch's de facto employment of "scientific pitch," or $\mathrm{C}=256 \mathrm{~Hz}$ (rather than the currently standard $261.63 \mathrm{~Hz}$ ) that allows one to perceive, via a specific tuning, the inner workings of the quartz crystal, whose resonating frequency is just another flat $\mathrm{C}$, out of hearing range, and whose microprocessor divisions by two transpose it into audibility and then back out of it, as it accurately calculates the length of a second. ${ }^{49}$

In comparing the digital watch and the mobile phone, history would seem to have repeated itself as tragedy and then farce, to invoke the now-clichéd dictum from Marx's The Eighteenth Brumaire. But if the mobile phone's ringtone was clearly the farcical repetition of a phenomenon past, what was tragic about the digital watch's beep? To revisit our initial discussion, perhaps it lies partly in the epochal routinization of the ubiquitous and now-residual "beep," found in numerous household, personal, and industrial devices-microwave ovens, washing machines, scanners, digital alarm clocks, automobiles, cellular phones, home computers - and which since the 1970 s were predominantly made by integrated circuits, small speakers, and simple, single-oscillator signals. The oscillator's beep, at one time, held 
a utopian promise, but the signal beeps from Sputnik now sound like an alarm clock; the conquest of nature and space that they represent once foretold of human betterment and today seems untenable. It is a state of affairs worthy of a cold, clear-eyed tear or two.

\section{Notes}

Many thanks to the individuals who graciously gave me feedback on or help with versions of this project, including Kate Altizer, Peter Burkholder, Phil Ford, Anand Gopinath, Sudhir Gopinath, Beth Hartman, Karen Ho, Marianne Kielian-Gilbert, Michael Klein, Ajay Limaye, Tom McAuley, Dan Melamed, Ali Momeni, Alex Monae, Tina Muxfeldt, Ayana Smith, Jason Stanyek, Jonathan Sterne, David Valentine, Christine Wisch, and the editors-with profuse apologies to those I've momentarily forgotten.

1. Oxford English Dictionary, s.v., "beep," https://oed.com/.

2. Edmund Wilson, I Thought of Daisy, ed. Neale Reinitz (Iowa City: University of Iowa Press, 2001 [1929]), 253-54.

3. A bicycle display ad for Gimbels department store lists "a small beep-beep French horn" for 74\$. New York Times, June 5, 1933, 6.

4. See Axel Volmar, "Productive Sounds: Touch-Tone Dialing, the Rise of the Call Center Industry and the Politics of Virtual Voice Assistants," in The Democratization of Artificial Intelligence: Net Politics in the Era of Learning Algorithms, ed. Andreas Sudmann (Bielefeld: Transcript, 2019), 55-75.

5. In 1957 a retailer in Kansas City, MO had the Sputnik beep recorded and pressed onto vinyl records, selling them for \$2.95. See Mark Shanahan, Eisenhower at the Dawn of the Space Age: Sputnik, Rockets, and Helping Hands (Lanham, MD: Lexington Books, 2017), 82. Thanks to Dan Melamed for mentioning Sputnik.

6. Peter E. Hunn, "CONELRAD," in Christopher Sterling, ed., Encyclopedia of Radio, vol. 1 (A-E) (New York: Fitzroy Dearborn, 2004), 605-607.

7. Bob Dylan mentions CONELRAD in the ninth verse of "Talkin' World War III Blues" (1963), and in the tenth verse he mentions dialing the operator and hearing "When you hear the beep it will be three o'clock"; the song juxtaposes two contemporaneously prominent beep contexts.

8. Ross Bassett, To the Digital Age: Research Labs, Start-up Companies, and the Rise of MOS Technology (Baltimore, MD: Johns Hopkins University Press, 2002), 1.

9. See Jeffrey Zygmont, Microchip: An Idea, Its Genesis, and the Revolution It Created (Cambridge, MA: Perseus Publishing, 2003), xviii and passim. For example, early personal computer speakers producing simple beeps were called "bippers" or "beepers"; see Karen Collins, Game Sound: An Introduction to the History, Theory, and Practice of Video Game Music and Sound Design (Cambridge, MA: MIT Press, 2008), 29. 
10. On the functional and historical differences between sound reproduction and synthesized sound, see Jonathan Sterne, The Audible Past: Cultural Origins of Sound Reproduction (Durham, NC: Duke University Press, 2003), 34-35.

11. Giovanni Arrighi, The Long Twentieth Century: Money, Power, and the Origins of Our Times (London: Verso, 2010).

12. I draw heavily on Amy Glasmeier, Manufacturing Time: Global Competition in the Watch Industry, 1795-200o (New York: Guilford, 2000), 88-106 (on Swiss watchmaking), 107-129 (on US watch production), 130-154 (on subsequent competition between the two), and ${ }_{155^{-177}}$ (on the Japanese watch industry).

13. This is in comparison to Britain. See Glasmeier, Manufacturing Time, 92.

14. Glasmeier, Manufacturing Time, 128.

15. Glasmeier, Manufacturing Time, 177.

16. Glasmeier, Manufacturing Time, 177; figures from Table 8.4 (174-175).

17. On the Japanese and US-American scientists, entrepreneurs, and companies involved, see Bob Johnstone, We Were Burning:Japanese Entrepreneurs and the Forging of the Electronic Age (Boulder, CO: Basic Books, 1999), 1-117. On Japanese economic competitiveness vis-à-vis the US in relative capital and labor costs during the 1970s-80s and decreasing US advantages in energy prices (due to the 1973 and 1979 energy crises), see Dale Jorgenson and Masahiro Kuroda, "Productivity and International Competitiveness in Japan and the United States, 1960-1985," in Productivity Growth in Japan and the United States, ed. Charles Hulten (Chicago: University of Chicago Press, 1990), 29-57, especially 38-41. The pre-OPEC oil crisis period (1968-1973) witnessed intensive Japanese $\mathrm{R} \& \mathrm{D}$ investment in primary electrical and electronics technologies, leading to a large accumulation of $R \& D$ stock (including in semiconductor microchip manufacturing) that propelled the digital watch market in the mid-1970s and after, continuing post-crisis and scaling upward more effectively in Japan than the US. See M. Ishaq Nadiri and Ingmar R. Prucha, "Comparison and Analysis of Productivity Growth and R\&D Investment in the Electrical Machinery Industries of the United States and Japan," in Productivity Growth in Japan and the United States, 109-133.

18. "Swatch," Wikipedia, http://en.wikipedia.org/wiki/Swatch, accessed October 21, 2019.

19. Perry Anderson, "Jottings on the Conjuncture," New Left Review II/48 (November-December 2007), 6 .

20. See, for example, Mizuko Ito, et al., eds., Personal, Portable, Pedestrian: Mobile Phones in Japanese Life (Cambridge, MA: MIT Press, 2005).

21. See Shaul Katzir, The Beginnings of Piezoelectricity: A Study in Mundane Physics (Dordrecht: Springer, 2006); also, see Takuro Ikeda, Fundamentals of Piezoelectricity (Oxford: Oxford University Press, 1996), 1-3; and "Quartz Clock," Wikipedia, http://en.wikipedia.org/wiki/Quartz_clock, accessed May 21, 2009. 
22. David Landes, Revolution in Time: Clocks and the Making of the Modern World (Cambridge, Mass.: Belknap Press, 1983), 342-3 and esp. 377.

23. See "Watch," Wikipedia, http://en.wikipedia.org/wiki/Wristwatch, accessed May 21, 2009; and Carlene Stephens and Maggie Dennis, "Engineering Time: Inventing the Electronic Wristwatch," British Journal of the History of Science 33 (2000): 492-4.

24. See the Bond film clip at http://www.oldpulsars.com/ as of October 26, 2019.

25. Glasmeier, Manufacturing Time, 209.

26. Ernest Braun and Stuart MacDonald, Revolution in Miniature: The History and Impact of Semiconductor Electronics, rev. 2nd ed. (Cambridge: Cambridge University Press, 1982), 181-183.

27. See the Rotary ad here https://www.youtube.com/watch?v=G4Zfwq_2hLk (accessed October 26, 2019) and the Seiko ad here https://www.youtube. com/watch?v=IrP79jWm3Qc (accessed October 26, 2019).

28. See "Making the Digital Watch Serve the Customer," in Tom Hyltin, The Digital Electronic Watch (New York: Van Nostrand Reinhold, 1978), 39-40.

29. Susan Kuzca, untitled, United Press International, 3 June 1981.

30. Pieter Doensen, Watch: History of the Modern Wristwatch:Design 1950-1983, Electric 1950-1993 (Gent: Snoeck Ducaju \& Zoon, 1994), 197; anonymous, Casio press release, PR Newswire, 12 June 1980.

31. Japan joined the newly formed G6 in 1975 with France, Italy, West Germany, the UK, and US.

32. “Q\&A: How Alarm Watches Make Their Noise," New York Times, 15 August 1989.

33. See Larry Humes, "What Is 'Normal Hearing' for Older Adults and Can 'Normal-hearing Older Adults' Benefit from Hearing Care Intervention?", The Hearing Review, July 14, 2020, https://www.hearingreview.com/inside-hearing/research/what-is-normal-hearing-for-older-adults, accessed September 6, 2020.

34. Indeed, $4096 \mathrm{~Hz}$ is close to the frequency with the lowest decibel threshold; see the chart in R. Murray Schafer, The Soundscape: Our Sonic Environment and the Tuning of the World (Rochester, VT: Destiny Books, 1994), 116. Thanks to Anand Gopinath, who suggested price determined the watch alarm signal's frequency and mode of amplification.

35. On the running boom, see Sumanth Gopinath and Jason Stanyek, "Tuning the Human Race: Athletic Capitalism and the Nike+ Sport Kit," in Music, Sound, and Space: Transformations of Public and Private Experience, ed. Georgina Born (Cambridge University Press, 2013), 128-148. Also see E. P. Thompson, "Time, Work-Discipline, and Industrial Capitalism," Past \& Present 38 (1967), 56-97.

36. James P. Sterba, "By 1990, Modern Quartz Will Have Nearly Silenced the Tick," New York Times, 9 February 1982.

37. Landes, Revolution in Time, 353; Richard Porch, "The Digital Watch: Tribal Bracelet of the Consumer Society," Design Issues 2/2 (Autumn 1985), 46.

38. Martin Smith (of the Orange County Register), "Technology's Timebomb Ticks," The Oregonian, 3 January 1989. 
39. Editorial, "Modern Times: Beeps," New York Times, 18 January 1982. In meetings during my father's employment at Pullman in the late 1970s-early 1980 s, watches would start to beep around the hour, and the presiding works manager would get annoyed and stop these meetings temporarily. Digital watches were typically owned by men; this may still be true today. (The gendering of the digital watch merits much greater study.) Sudhir Gopinath, conversation on August 21, 2013.

40. Glasmeier, Manufacturing Time, 255. Moreover, as luxury goods, mechanical watches made a comeback. See Joe Thompson, "Mechanical Watches Almost Disappeared Forever. Here's How They Didn't," Bloomberg, 4 January 2018, https://www.bloomberg.com/news/articles/2018-01-04/ how-mechanical-watches-survived-after-quartz-a-concise-history, accessed September 1, 2020.

41. Carlene Stephens and Maggie Dennis, "Engineering Time: Inventing the Electronic Wristwatch," British Journal for the History of Science 33 (2000), 497.

42. See Mike Murphy, "The Apple Watch is a Great Smart Watch and Finally a Good Dumb One Too," Quartz, 23 October 2019, https://qz.com/1719176/theapple-watch-series-5-is-the-best-one-yet/, accessed October 26, 2019.

43. Older smartwatches were peripherals, dependent on stronger devices. Susan Ryan, Garments of Paradise: Wearable Discourse in the Digital Age (Cambridge, Mass.: MIT Press, 2014), 235.

44. Annie Van Cleve, "A Time for Youth: Wristwatch Losing Ground in HighTech World," The Capital Times (Madison, WI), December 1, 2006, http:// watch-talk.blogspot.com/2006/12/wristwatch-losing-ground-in-high-tech. html, accessed 9 September 2014. Also see "Smartwatch," Wikpedia.org, http://en.wikipedia.org/wiki/Smartwatch, accessed October 22, 2019.

45. Landes, Revolution in Time, 365. After WWII, "cheap time" was purveyed most by US-based budget watchmaker Timex.

46. See my The Ringtone Dialectic: Economy and Cultural Form (Cambridge, MA: MIT Press, 2013).

47. See Larissa Hjorth, "Odours of Mobility: Mobile Phones and Japanese Cute Culture in the Asia-Pacific," Journal of Intercultural Studies 26 (2005), 39-55; and Sianne Ngai, Our Aesthetic Categories: Zany, Cute, Interesting (Cambridge, MA: Harvard University Press, 2012), 77-86.

48. See Melissa Gregg and Tamara Kneese, "Clock," in Timon Beyes, et al., The Oxford Handbook of Media, Technology, and Organization Studies (New York: Oxford University Press, 2019), 95-105.

49. Scientific pitch, present in older digital casino games and slot machines (also tuned to scientific $\mathrm{C}$ ) as well as digital watches and alarms, is an underappreciated facet of the auditory culture of an older digital modernity. Thanks to Dan Melamed for this argument. Also see Bruce Haynes, A History of Performing Pitch: The Story of 'A' (Lanham, MD: Scarecrow Press, 2002), 42, and "The Science of Music: What Is Beauty, and Why Is It Necessary?", The Schiller Institute, n.d., https://archive.schillerinstitute.com/ music/rev_verdituning.html, accessed October 22, 2019. 


\section{About the Author}

Sumanth Gopinath is Associate Professor of Music Theory in the School of Music at the University of Minnesota. His research interests include musical minimalism, sound studies, new media, and experimental and popular music. He is the author of The Ringtone Dialectic and co-edited volumes on mobile music and Steve Reich. 


\section{Part IV}

Futures 



\title{
13. Captured Time: Eye Tracking and the Attention Economy
}

\author{
Alexander Monea
}

\begin{abstract}
This chapter examines the history how eye tracking came to stand in as a measurement for what people pay attention to. I argue that this connection between eye movement and attention is problematic and may lead to undesirable developments in the contemporary attention economy as it is implemented across digital platforms and smartphone apps in the near future-e.g., ads that pause playing when you look away from the screen. The chapter traces the emergence of eye tracking technology in the late nineteenth and early twentieth centuries and shows how its implementation to measure attention in the mid twentieth century cemented its purpose and has driven its development ever since.
\end{abstract}

Keywords: eye tracking, surveillance, attention, human-computer interaction, media studies, history of technology

It is curious that today there are nearly as many popular authors arguing that attention can be captured and sold reliably enough to form the bedrock of the contemporary economy as there are authors arguing that with self-discipline we can harness our attention to either succeed in or escape from that very same contemporary attention economy. ${ }^{1}$ The collective wisdom across these texts is something like the following: when an individual mechanism of attention capture works, it's zombifying capacities are near inescapable, but any individual mechanism of attention capture will not be very efficient and will be highly exploitable, subvertable, or even just ignorable. As such, the infrastructure of the attention economy has been one of pure bombardment, of inefficient spamming, which when collectively constant and immersive ensures that you are everywhere and

Volmar, A. and K. Stine (eds.), Media Infrastructures and the Politics of Digital Time: Essays on Hardwired Temporalities. Amsterdam: Amsterdam University Press, 2021 DOI 10.5117/9789463727426_CH13 
always ensnared by at least one or two of these mechanisms while you ignore the rest. There is thus a fundamental ambiguity in the term. Our attention has become both our greatest weakness and the source of our salvation in the information age.

The reason that attention presents us with this paradox today is because of its historical fusion with vision. It is this assumption - that the movement of the eyes constitutes a sufficient stand-in for mental focus - that renders the temporality of attention available for quantification and thus, in the terms of Lorraine Daston and Peter Galison, "mechanically objective" knowledge. ${ }^{2}$ As we'll see, the slippage between the qualitative temporality of everyday attention and the quantitative temporality of tracked eye movements is one reason for this paradox - the second will be the radical unpredictability of eye movements. Nonetheless, our culture is dominated by an idea that the patterning of lived temporal phenomena can be put into a circulatory relationship with what media theorists like Axel Volmar or Wolfgang Ernst have described as the microtemporalities of time-critical media like eye tracking sensors and the flow of electrons across the silicon circuit boards of computational devices. ${ }^{3}$ Through the mediation of eye tracking, the thoughts of mind and machine might be tethered across their temporal registers. Mind and machine might be made to correspond with and to pattern each other, which, in our most dystopic nightmare and the wildest dreams of platform shareholders, might lead to a command and control structure.

The first section examines the history of eye tracking technologies from their conception in the nineteenth century to their stabilization in the twentieth century. By mid-century, eye tracking technology was accurate, available, and affordable - it was a hammer waiting for a nail, so to speak. The second section shows how eye tracking came to be nearly synonymous with tracking attention. Cyberneticists and psychologists looking to measure attention turned to eye tracking as the only objective metric available for attention. This cybernetic legacy continually inspires the rhetoric in which eye tracking is positioned as a mechanism of command, control and communication. While this representation has always been fraught, eye tracking research often slips back into a reliance on science fiction as it acknowledges but then quickly forgets its own limitations. The final section argues that eye tracking technology currently constitutes the largest gap in the attention economy and that contemporary technologies make it increasingly feasible that attention merchants will try to capture that data. I posit two dystopic futures, one soft and one hard. In the soft dystopia, attention merchants are able to capture eye tracking data to, for instance, 
make sure we pay for freemium content with our attention to advertisements. In the hard dystopia, the specificity of each individual's eye movements will become machine learnable and thus dynamic design processes will be able to modulate our perception — and perhaps attention-in a much more consistent way.

The future of the attention economy from digital platforms to smartphone apps could be inflected by the advent of eye tracking technology being placed by default in all our screen-based interfaces. The use of ubiquitous eye tracking technologies to pattern the temporal flow of attention will likely constitute a speed-up and convenience for some users while constituting a slow-down and burden for others. It is easy to imagine a future where affluent users access premium dynamic interfaces that increase their speed and productivity while a large portion of less privileged users have their attention trapped and monetized in exchange for access to apps, platforms, or the internet writ large-think Zuckerberg's "free" internet (via drones, satellites, or balloons) on steroids.

\section{The Mechanization of Eye Tracking, or Why the Eye Can't be Controlled}

Throughout the nineteenth century many attempts were made to measure eye movement. Louis-Émile Javal, for instance, used afterimages to determine that there was no vertical displacement of the eye as it glided horizontally across text during reading. ${ }^{4}$ This experiment is popularly understood as the origin point of modern eye tracking. A number of contemporaneous researchers were considering using feathers and bristles attached to the eye to record its movements. ${ }^{5}$ By the turn of the century, Edmund Burke Huey published a groundbreaking study on the psychology of reading. Huey had successfully used plaster-of-Paris to build a mold that fitted over the cornea that was connected by a light celloidin-covered glass lever to a kymograph (fig. 13.1). The glass lever maneuvered the aluminum pointer of the kymograph to record its movements on a smoked drum, and later on smoked paper, as subjects read varying texts (fig. 13.2). Huey writes, "During the reading, the reader was usually quite unconscious of there being an attachment to his eye, and the reading proceeded as glibly and easily as could be desired," perhaps because he rendered participants' eyes insensitive by the application of holocain or cocaine. ${ }^{6}$ Provided the subject's head was fastened between iron standards to keep it still, he could successfully record their eye movements (fig. 13.1). Thus, by limiting the 


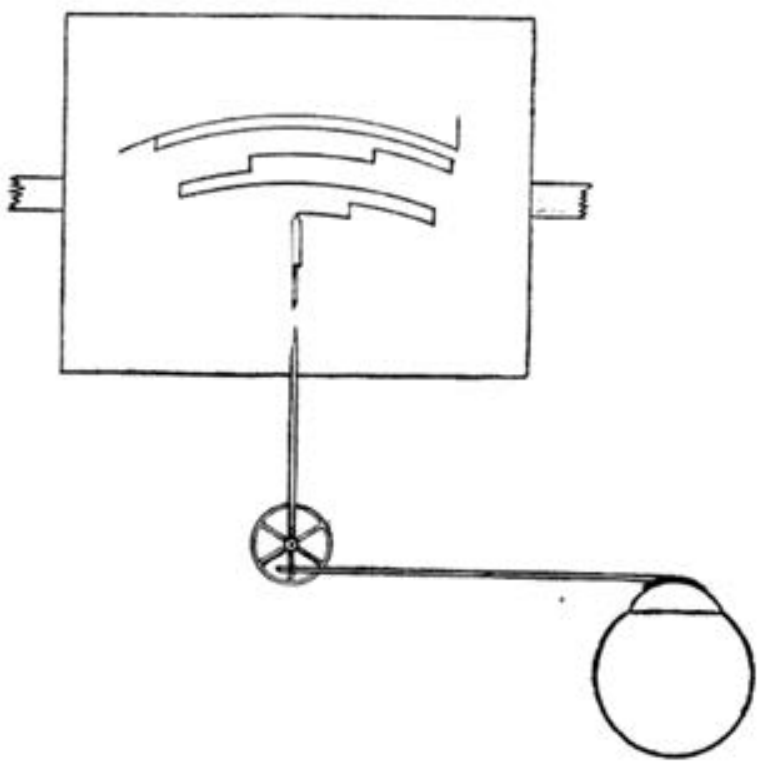

Figure 13.1. Huey's eye tracking apparatus

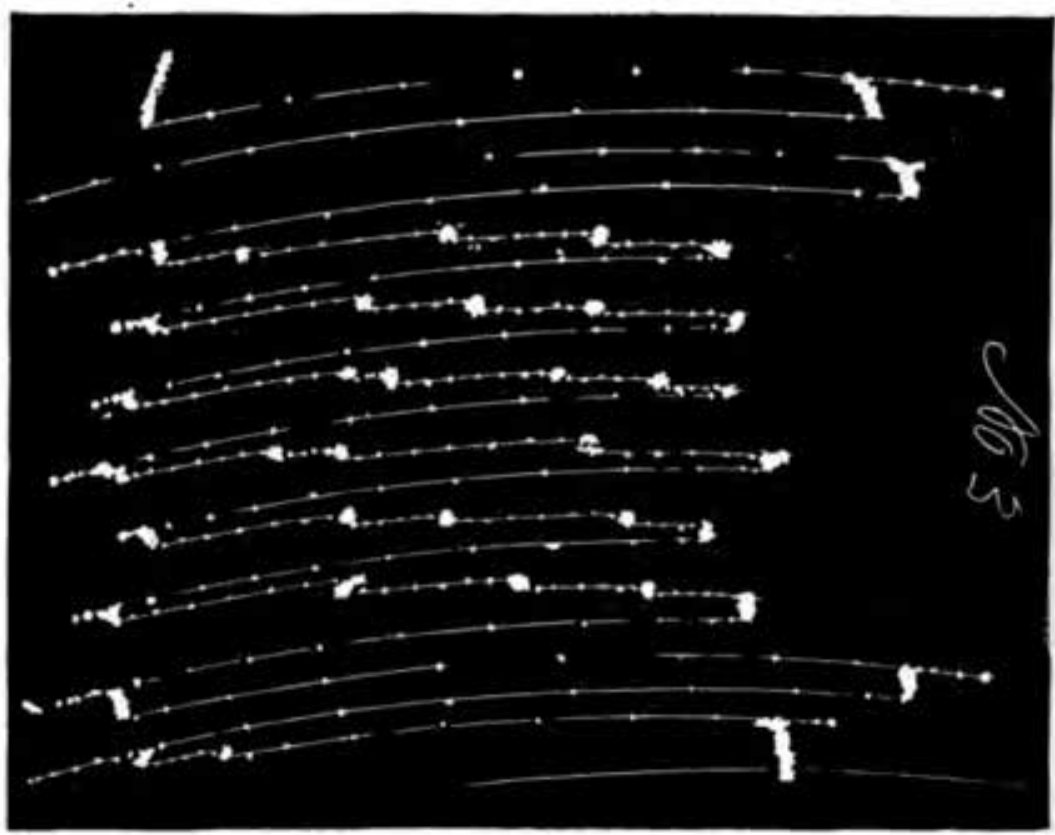

Figure 13.2. Scanpaths recorded by Huey 
subject's mobility in space, Huey could capture the foci of the subject's eye movements in time, thus allowing what would retrospectively be enshrined as one of the first successful synchronizations of mind and machine.

Despite Huey's claims, the mechanical properties of his apparatus limited its ability to objectively capture eye movement, in particular because the inertia from the apparatus caused overshoots in the recorded traces. ${ }^{7}$ In 1901, Raymond Dodge and Thomas Cline wrote, "Out of this advance in the physiology of vision has developed a constantly growing group of psychological questions which only a quantitative knowledge of the eye movements can answer." ${ }^{8}$ Dodge and Cline worked to develop an apparatus capable of making exact measurements of eye movements, with a particular focus on their angle velocity. A successful apparatus would need to register eye movement without producing momentum or inertia. ${ }^{9}$ The only possible solution was film, the perfect medium for turning a sequence of captured spatial coordinates into a simulation of phenomenological temporality. In a move that would determine many of our contemporary eye tracking technologies, Dodge and Cline would catch reflected light off the cornea on a sensitive film. In an apparatus jerry-built with knitting needles, bicycle pumps, cardboard, and a modified $5 \times 7$ bellows camera, they would secure their subject's head and photograph their eye movements (fig. 13.3).

Their most important invention was a plate-holder inside the camera that would move the film vertically in a continuous and even motion immediately behind a narrow horizontal slit through which the reflected light off the cornea was received (fig. 13.4). In addition, they used a pendulum within the plate-holder to create a time record by having its oscillations periodically intercept the light allowed in by one side of the horizontal slit in the plate-holder (fig. 13.5).

In later studies, Dodge would come to call these exposures 'kinetograms' (fig. 13.6). ${ }^{10}$ With these kinetograms, a mechanically objective representation of the temporality of eye movements became available to researchers. The reliability and cost effectiveness of this new medium far exceeded the limited demands of eye tracking research to that date, still largely focused on reading and visual impairments. It was, in a sense, a hammer awaiting a nail that would not appear until eye movements became a stand-in for human attention writ large. While this conjuncture would be instituted largely by psychologists outside of opthamology laboratories, the stage was set by researchers who moved from analyzing scanpaths during reading to scanpaths during analysis of images. 


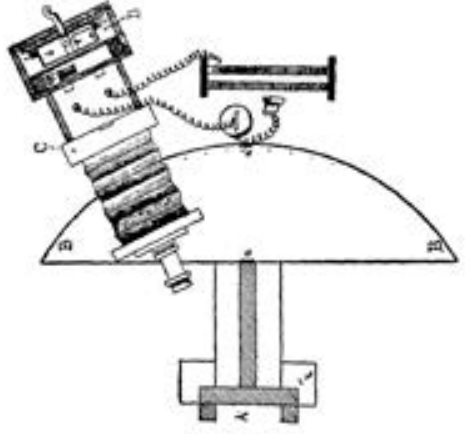

Figure 13.3. Dodge and Cline's eye tracking apparatus

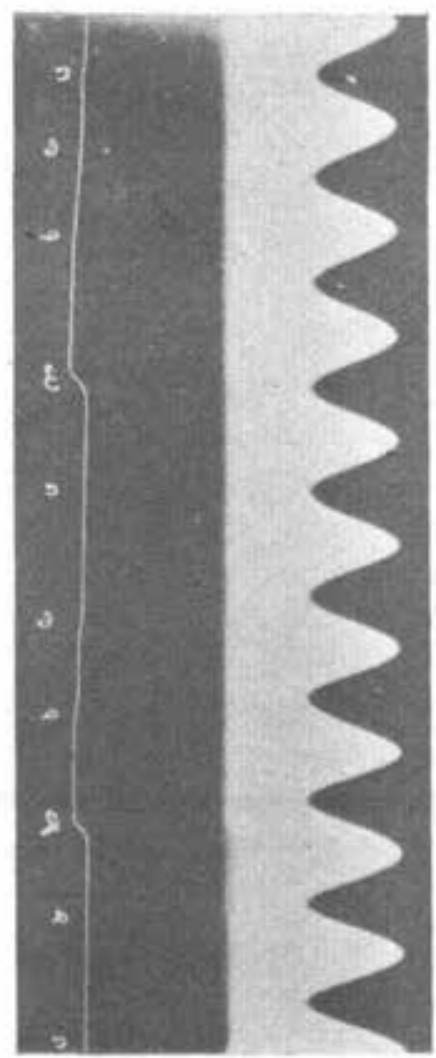

Figure 13.5. Dodge and Cline's film recording of eye movements

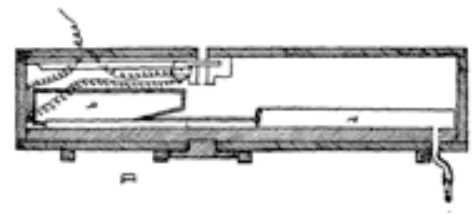

Figure 13.4. Dodge and Cline's film box

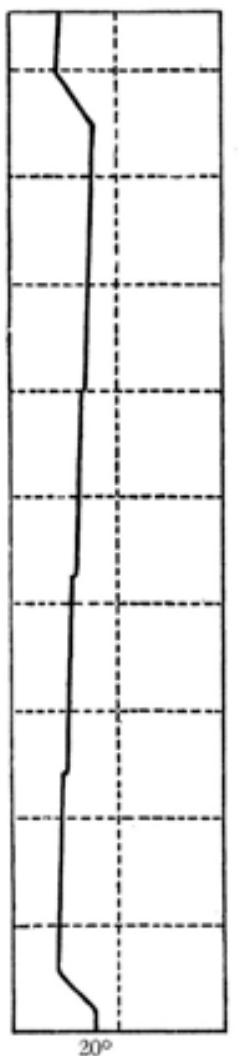

Figure 13.6. Dodge and Cline's film recording of eye movements 


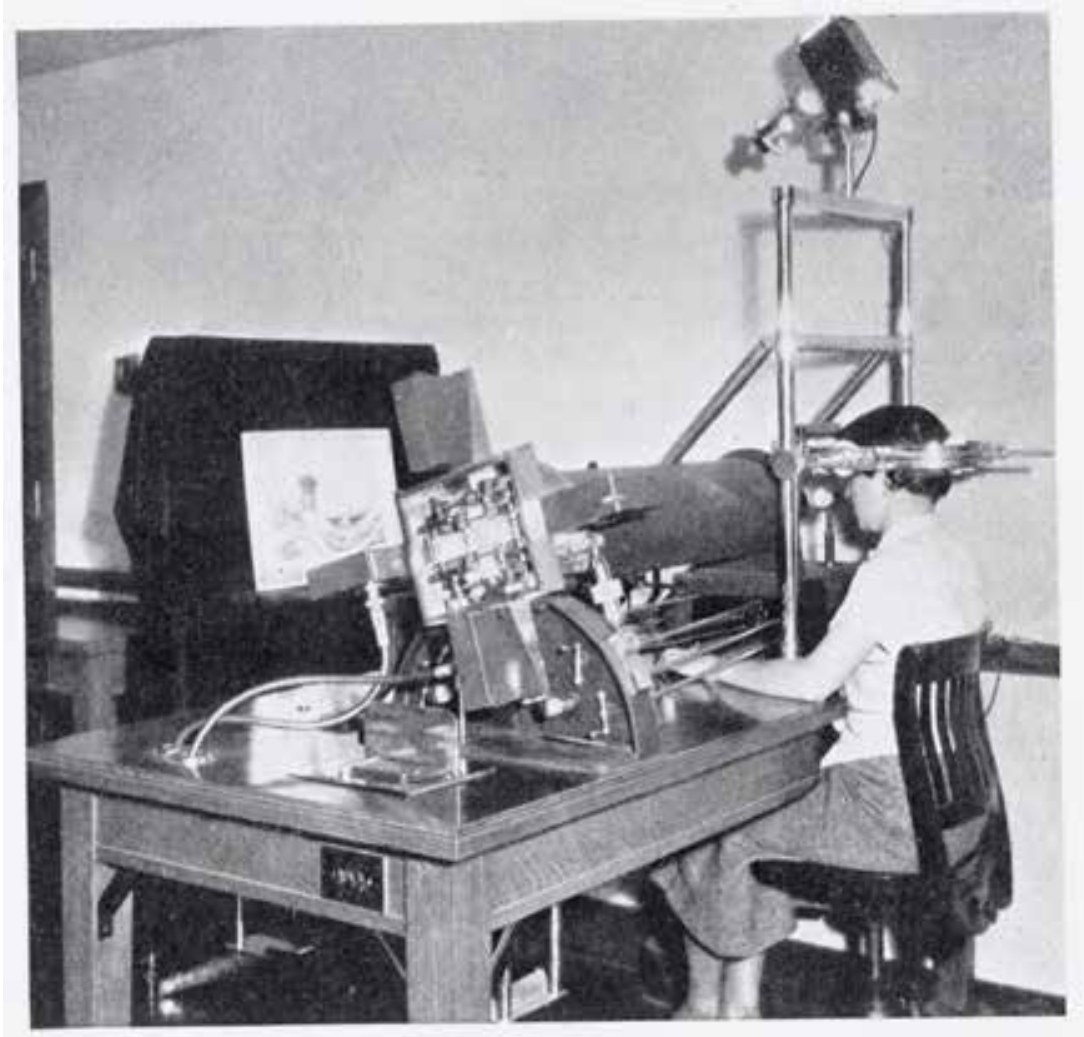

Figure 13.7. Buswell's eye tracking apparatus

The use of film to capture eye movements became the norm in eye tracking research for nearly 70 years and was quickly applied outside of the study of reading. George Malcolm Stratton would use a similar photographic technique to examine eye movements when subjects viewed simple geometrical patterns and line illusions. Stratton quickly found that he could reliably predict the scanpaths that his subjects' eyes would take when presented with these patterns, ${ }^{11}$ and was particularly unnerved by how disharmonious eye movements could produce the aesthetic experience of symmetry. ${ }^{12}$ As Wade notes, "Stratton's work is significant because it attempted to bridge the gap between visual phenomena (illusions), cognition (aesthetic judgments), and the underlying mechanisms (eye movements)," and further because Stratton shifted the focus of scientific study from the movement of the eyes to the locations selected for fixation. ${ }^{13}$ This focus on how we select particular locations to fixate on would become a central research question 


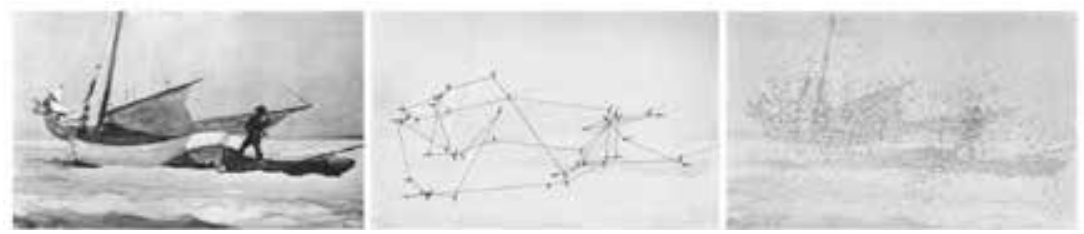

Figure 13.8. Buswell's analysis of eye movements during viewing of painting

moving forward and would set the stage for the fusion of eye movement and attention research.

The next major advancement came in 1935 when Guy Buswell published the eye tracking records of nearly 2,000 individual scanpaths from 200 participants each of whom viewed multiple pictures through an apparatus very similar to Dodge and Cline's (fig. 13.7) ${ }^{14}$ Rather than focusing on the eye movements themselves, Buswell analyzed the fixation points, publishing density plots from all of his recorded scanpaths (fig. 13.8). By reducing the spatial mobility of the subject's head, Buswell was able to use film to capture the temporal dimensions of eye movements as sequences of fixations. This reduction then allowed for a spatialization of these temporal dimensions (fig. 13.8).

From this innovative work, Buswell made some startling discoveries about the uniqueness of his participants' scanpaths. People tended to have very different scanpaths when presented with the same picture, while only the first handful of fixation points had even a semblance of homogeneity. Additionally, the average duration of fixations for each picture varied widely across observers. Buswell examined whether these differences correlated well with other variables like artistic training, age, and race/ethnicity, but found that within group variation always exceeded the average variation between groups to such an extent that the correlations could not be considered significant. ${ }^{15}$ In essence, people's phenomelogical temporalities were radically individualized. While mechanically objective recording of eye movements was now able to synchronize with the flow of phenomenological vision in its past and present foci, it remained unable to predict future foci.

Alfred L. Yarbus would eventually extend this research by fusing the film recordings with suction cup eye devices to investigate miniature eye movements during fixation, detailed kinematics of individual saccades, vergence, pursuit, and, perhaps most importantly, eye movements during perception of complex objects. ${ }^{16}$ For this last emphasis Yarbus famously analyzed the eye movements of subjects as they viewed Ilya Repin's painting The Unexpected Visitor. He found that while there was some homogeneity in the scanpaths, especially in the earlier points of fixation, the scanpaths were highly individualized. Additionally, when the same subject viewed 

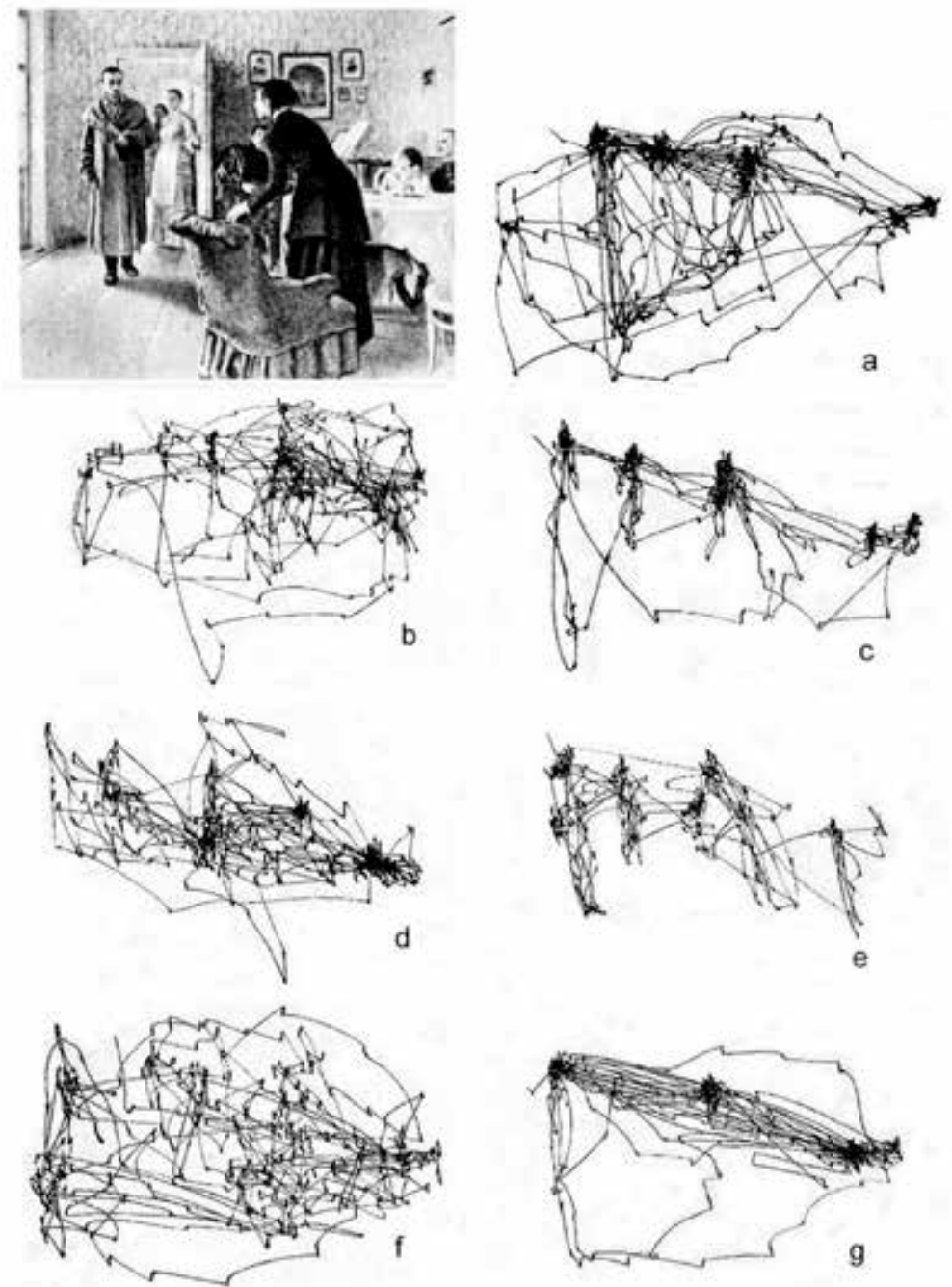

Figure 13.9. Yarbus's analysis of scanpaths when viewing painting based on priming.

the same painting a number of separate times, each successive viewing's scanpath would be different, although the difference in scanpaths was greater between different subjects than between successive viewings by the same subject. ${ }^{17}$ In perhaps the most enduring illustration of early eye tracking research, Yarbus extended Buswell's research into priming's effect on scanpaths (fig. 13.9). As Yarbus notes, "Depending on the task in which a 
person is engaged, i.e., depending on the character of the information which he must obtain, the distribution of the points of fixation on an object will vary correspondingly, because different items of information are usually localized in different parts of an object." 18

The role of priming and other cognitive factors in determining scanpaths, and in particular the sequence of selected points for fixation, is still central to eye tracking research and difficult to predict with much accuracy. ${ }^{19}$ Yarbus' study was recently reproduced and expanded to demonstrate that what he found after priming a single subject upon multiple viewings of The Unexpected Visitor was generalizable to multiple subjects. ${ }^{20}$

\section{Cybernetic Analysis of Black Boxes, or Why People Try to Control the Eye}

It is in Paul Fitts' research from the 1940s that eye tracking first was welded to human attention. Here the foci of eye movements stand in for the foci of mental attention and thus the scanpaths give an approximation of the sequence and rhythm of human thought-particularly as a stream of consciousness. Fitts was a pioneer in human factors, served as the sixth president of the American Psychological Association, and developed important laws and formulae for how humans use visual and proprioceptive feedback to situate themselves in space and time. He explicitly positioned himself in a tradition of interdisciplinary military research that was meant to better adapt humans and machines for cooperative interaction in combat, with a particular focus on aviation. ${ }^{21}$ The goal of much of this collaborative research between the American Psychological Association and the military was to better select and train pilots, better design aviation technology (esp. cockpits), and thus to ensure optimal air force capacities. For Fitts, this was fundamentally an informational problem about the visual and proprioceptive feedback loops that allow a pilot to interact with an aircraft. ${ }^{22}$ This approach situates Fitts in a transitional discursive tradition between behaviorism and cognitivism that Paul Edwards has described as "cybernetic psychology."23

Behaviorism understood the human mind to be a black box that could only be studied through measuring its inputs (stimuli) and outputs (behavior) with the ultimate goal of producing a stable theory of the functional relation between the two such that behavior might be controlled through the engineering of stimuli. Cybernetics largely shifted the metaphor of the black box onto electronics and used information theory to produce new methods of inferring the contents of black boxes through analysis of random noise inputs, and then 
used the analogy of mind and machine to argue that the same mathematical analyses useful in understanding electronics could similarly be applied to the human mind. ${ }^{24}$ The goal of cybernetic psychology was "to understand the processes of perception, memory, and language in terms of formalizable transformations of information and feedback circuits or control loops."25

While some psychologists directly equated human attention with a mechanical model of sensory inputs, ${ }^{26}$ Fitts was more open about the fact that the connection between thought, attention, and vision was more a matter of convenience. He writes, "If we know where a pilot is looking we do not necessarily know what he is thinking, but we know something of what he is thinking about. In servo terminology, we know what error signal inputs he is operating on."27 What the eye is focused on is only roughly correlated to what the mind is paying attention to, which in turn is only roughly corelated to what the mind is thinking about. Eye movements and fixations were chosen as inputs to be studied because they were the only variables that could be objectively observed and mechanically reproduced. In short, eye movements became the stand-in for attention - and thus the temporality of thought - because eye tracking technology was sophisticated, affordable, and accurate enough to serve scientific and military purposes.

This is the point of fusion between a technology waiting for an application and a theory of mind looking for a method. This entanglement of thought, attention, and vision became essential to cybernetics as a whole. ${ }^{28}$ Take, for example, the cybernetic understanding of teleology outlined by Arturo Rosenblueth, Norbert Wiener, and Julian Bigelow. ${ }^{29}$ They understood humans and machines to pursue goals in the same way, by utilizing a cycle of predicting future states, taking actions, and self-correcting their course of action based on negative feedback received. Goals are "a final condition in which the behaving object reaches a definite correlation in time or in space with respect to another object or event." ${ }^{30}$ The sensory feedback received is primarily visual. Warren McCulloch went so far as to argue that the mind was dominated by the eye. He writes, "The eye is not only the most important of sense organs. It is the most complicated, being in reality an invaginated evagination of the brain itself." ${ }^{11}$ Here McCulloch is arguing that in vision the brain is externalized and folded back in on itself; it touches itself as it touches the world. This is no more nor less than the cybernetic version of Maurice Merleau-Ponty's insights about the chiasmthe entanglement of sensor and sensation - whose anatomical definition interestingly corresponds to the crossing optic tracts of the eyes that is so fundamental to binocular vision..$^{2}$ As Rudolf Arnheim has shown at great length perception contains cognition, or as he puts it, "visual perception is 
visual thinking." 33 This is central to cybernetic psychology: we must examine sensory inputs - vision in particular - if we are to understand how the mind works as a mechanism. In the context of aviation, as Fitts notes, "we cannot study man's motor system at the behavioral level in isolation from its associated sensory mechanisms." ${ }^{34}$

Eye tracking research continues to bear the imprint of this cybernetic legacy today. The textbook Eye Tracking Methodology describes the visual system repeatedly in terms of information processing, noise reduction, command and control systems, and feedback circuits and loops-including as an "attentional feedback loop." ${ }^{35}$ While the author is careful to note that he is speaking of visual attention and not attention writ large, the analogizing of mind and machine leads him to habitually conflate the two in practice. For instance, Duchowski refers to eye tracking data as "objective evidence provided by users' gaze and hence attention.. ${ }^{36}$ Wilson Geisler and Lawrence Cormack similarly describe attention with a cybernetic vocabulary, and Árni Kristjánsson goes so far as to argue that eye movements and attention largely share the same neural resources..$^{37}$ This is a discursive convention that companies producing eye tracking technologies like Tobii are all too happy to encourage. In their online marketing materials for Tobii Pro they write, "Eye tracking is a unique method to objectively measure consumers' attention and spontaneous responses to marketing messages." ${ }^{38}$

As I've shown, this repeated analogizing of mind and machine was embedded in eye tracking discourse through cybernetics in the mid-twentieth century. The result of that legacy is that we are led to believe that human "systems" might be just as amenable to command, control, and communication as computational systems. However, as I've shown in the previous section, our scanpaths are too susceptible to priming and other idiosyncrasies for this analogy to ever hold true. Instead, just as Colin Milburn has shown in the context of nanotechnology, eye tracking is as much science fiction as science. ${ }^{39} \mathrm{By}$ this I do not mean that there is no real science behind the use of eye tracking. There is a great deal of innovative and rigorous research being conducted through these means. What I do mean is that the investment in and development of eye tracking technologies is as much driven by science fiction stories about successful applications in the future as it is by current applications. Regardless of the success or failure of any of these anticipated future applications, the discourse on eye tracking will continue producing science fiction so long as its cybernetic genealogy remains intact. Eye tracking seems forever destined to fail to live up to its hype because of the individual specificity of eye movements. Yet, like it is our political party or our compulsively philandering spouse, we can't help 
but believe its perpetual promises that it has turned a corner, that this time it will be different, that things are finally going to work out.

\section{Computational Eye Tracking and Two Dystopic Futures}

In 2016, Nvidia publicly announced ongoing research into the use of eye tracking technology to make gains in computer processing power for virtual reality. By taking advantage of foveal vision, Nvidia realized it could drastically reduce the processing power required to render VR graphics. In essence, Nvidia argues that VR headsets can render the graphics outside a designated range of the current eye fixation in lower resolution without the user noticing any ill effects. This may prove essential for the future of VR. As Simon Parkin writes, "When the player using the Nvidia system focuses on a new area of the scene, eye-tracking software shifts the focus of the rendering in kind. To render a full scene in VR at 90 frames per second, the lowest acceptable frame rate in VR before users begin to report feelings of nausea, four million pixels must be rendered at almost a hundred times a second. But by focusing the rendering only on the player's line of sight, huge computational savings can be made. ${ }^{.00}$ While Nvidia will not produce VR headsets with eye tracking technology, a number of companies are already moving in this direction. For example, the Kickstarter-backed Fove headset is meant to be completely controlled by the user's eye movements, ${ }^{41}$ and companies like SensoMotoric Instruments and Tobii Tech are already working to adapt their eye tracking technologies to VR headsets. As people increasingly access visual information through VR media, the natural inclusion of eye tracking technology promises to offer a fresh new stream of user data.

As far back as 2011, Tobii was partnering with Lenovo to produce a laptop that could be controlled with eye motions, but these technologies have yet to sweep the consumer market. ${ }^{42}$ This may in part be due to the high cost, need to accommodate cumbersome hardware, and inaccuracy during real-world use that such systems have demonstrated. Recently we have seen rapid advances in software-based solutions that take advantage of preexisting technologies, like webcams and forward-facing cameras on tablets and smart phones, whose fixed position relative to the screen can be exploited for eye tracking purposes. ${ }^{43}$ Machine learning has left eye tracking technology largely unimproved, ${ }^{44}$ and this is likely due to limitations in the availability of large-scale datasets of captured eye movements - most have around 50 subjects. ${ }^{45}$ By 2016 , researchers were constructing "a mobile-based eye tracking dataset containing almost 1500 subjects from a wide variety of 
background, recorded under variable lighting conditions and unconstrained head motion.."46 They fed this data into a convolutional neural network and produced a system that could match state-of-the-art eye tracking performance. Further, they were able to use dark knowledge approaches to simplify their model enough for it to run in real-time on mobile devices. ${ }^{47}$ Provided the ubiquity of eye tracking technology is solely a software problem, it is safe to assume that we are not far from eye tracking technology being a standard feature of all smartphones, tablets, and laptops.

The soft dystopic vision of the future is one in which the current trend of using click-through, typed input, scrolling, and time-spent-on-page data to make these attention capture mechanisms more efficient is supplemented with eye tracking data at web scale. Once our VR headsets, laptops, tablets, and smartphones incorporate eye tracking technology, it is inevitable that our eye tracking data will be collected as we navigate our digital worlds in exchange for access to new digital goods and services. The collection of vast stores of user scanpaths offers up a first solution to the individuality of our eye movements: we then might collect enough data per user to statistically model their individual eye movement behaviors in response to various stimuli and priming. As user interfaces and designs become increasingly modular and dynamic, it is not hard to envision a world in which this collection of our eye movements can lead to the personalization of our interfaces through ongoing dynamic iterations. For instance, every Google account, or even every Google search for every Google account, would generate a particular optimal placement for "sponsored content" so that it might draw the most attention and produce the most click-throughs. This will never be a world in which we become perfect ideological drones ready for ongoing hypnotic commands from global capital, but it will be one in which the effectiveness and efficiency of our current attention capturing mechanisms is vastly increased.

Even should dynamic or personalized interface designs not prove possible with eye tracking data at web scale, there are even easier to imagine dystopic futures that can arise when eye tracking technology is embedded in all of our screen technologies as a default. The business models of most of our digital platforms and the bulk of our "freemium" smartphone apps are based on advertising revenue. Imagine a world in which these companies could monitor the consumptive labor of viewing advertisements that their users are performing for them. Imagine a world in which advertisements pause when you stop looking at them. Where you can't return to primary content until you've paid attention. Imagine how much more these companies could charge advertisers if they could guarantee eyeballs on their adds, literally. We 
aren't far away from a Candy Crush app that tracks your eye movements to make sure you are watching the advertisements that unlock daily boosters, or from public WiFi hotspots that require you to focus on intermittent advertisements to stay connected. The most likely dystopia is one in which consumptive labor-i.e. watching advertisements-becomes a stable market and attention gets standardized into currency that you pay with. Here the phrase "paying attention" will finally take on its full meaning under capital.

In this first dystopic vision of the future one can already see the potential emergence of even greater class and gender differentiation in the temporal patterning of digital flows. As Axel Volmar and Kyle Stine have pointed out in their introduction to this volume and Sarah Sharma has analyzed in great detail, ${ }^{48}$ there already exists a great class differentiation in our contemporary lived temporalities, as the optimization and acceleration of affluent people's everyday lives is a privilege borne on the backs of the underprivileged, whose lived temporalities are relationally overburdened, disrupted, and thrown out of sync with the world. We already have an instance akin to my imagined dystopia in the use of a platform like YouTube, which serves more ads to people accessing the site via mobile phones than on desktops and laptops - and keep in mind that the underprivileged people of the globe are much more likely to only have internet access through their mobile devices. One can easily envision the exacerbation of these divides by imagining the future of affluent, white, cis-gendered men paying to use optimized interfaces in the future to speed up their digital lives on platforms subsidized by the attentional labor of, for instance, working class, black, single mothers forced to pay attention to every second of the ads supporting their access to digital platforms. This situation will only be worse in areas of the globe without affordable broadband access. As platforms like Facebook look to offer drone, satellite, and balloon-based mobile internet access to their closed iterations of the "internet," it is easy to imagine a future in which these people's entire internet access is facilitated by the extraction of attentional capital through eye tracking technologies. In this future, the digital temporality of large swaths of the globe might be patterned in accordance to these attentional extraction mechanisms.

In the hard dystopic vision of the future is one in which this process is much more successful than can be reasonably hoped for. Here we can envision a process whereby the personalization afforded by eye tracking data will lead to completely individualized interfaces and designs statistically optimized to capture attention by structuring the sequence of points of fixation in our scanpaths. This is a literal determination not only of what but of how we see. In his book Visual Thinking, Rudolf Arnheim argues that 
the "cognitive operations called thinking are not the privilege of mental processes above and beyond perception but the essential ingredients of perception itself," and also that "visual perception ... is not a passive recording stimulus material but an active concern of the mind." ${ }^{49}$ As such, we can understand such a capacity for dynamic iterative design based on personalized scanpath profiles to be a literal manipulation of mind. This hard dystopia fits in well with a long tradition in film criticism ranging from Horkheimer and Adorno's woes over cinema relieving of us of any capacity to imagine for ourselves to William Connolly's more recent arguments that the cinema can utilize affect to (re-)program its audience..$^{50}$ The primary difference here is that this will be a world in which the mechanism of attention capture is individualized beyond all possibility of communal experience, and thus short-circuits the capacity for building communities through shared experience. In his three-volume work Technics and Time, Bernard Stiegler worried that mass media have led to a world in which everyone's lived temporality is synchronized with broadcast media in what he terms "the industrial temporalization of consciousness," thus leading to a cultural leveling and homogeneity. In this hard dystopia we can see the opposite problem arising from network media and attentional modulation, where every single person's lived temporality is synchronized with a radically individualized temporal patterning mechanism..$^{1}$

The endpoint of eye tracking certainly does not need to be so grim. It is extremely promising as a method for creating user interfaces that make attention less intentional, so to speak, and thus less exhausting. This is precisely the promise that Walter Benjamin saw in film: in opposition to Horkheimer and Adorno, Benjamin argued that film was uniquely capable of "distracted reception," where audiences could develop a critical understanding of the film's contents without having to expend the conscious energy of intentionally directing their attention towards each nuance of the film's contents. ${ }^{2}$ Personalization based on scanpath models could do something similar by making objects we need or ought to focus on easier to pay attention to - more absorbing, interesting, or intuitive. It could be the visual equivalent of Richard Thaler's nudges..$^{53}$ It might make learning or communicating much easier or more efficient for many more people. The problem is with the potential weaponization of this data, which seems all but inevitable given the attentional infrastructure which such a technology would get plugged into. In such an instance, what used to be our primary defense against attention capture-namely, that eye movements and fixations were highly individualized with no clustering of behavior around demographic groupings-becomes our new primary weakness, which is 
the personalization of attentional objects. Perfect echo chambers. Mass solipsism. Catatonia. A horrific monadology. The true boob tube. A real-life version of Ringu in which the husks of departed souls are found slouched before screens that just read as static to second parties.

\section{Notes}

1. For evidence of the former, see Thomas H. Davenport and John C. Beck, The Attention Economy (Cambridge, MA: Harvard Business Review Press, 2001); Mara Einstein, Black Ops Advertising: Native Ads, Content Marketing and the covert World of the Digital Sell (New York: OR Books, 2016); Nir Eyal, Hooked: How to Build Habit-Forming Products, ed. Ryan Hoover (New York: Penguin, 2014); Ben Parr, Captivology: The Science of Capturing People's Attention (New York: HarperOne, 2016); Jonathan Taplin, Move Fast and Break Things: How Facebook, Google, and Amazon Cornered Culture and Undermined Democracy (New York: Hachette, 2018); James G. Webster, The Marketplace of Attention: How Audiences Take Shape in a Digital Age (Cambridge, MA: MIT Press, 2016); Tim Wu, The Attention Merchants: The Epic Scramble to Get Inside Our Heads (New York: Vintage, 2017). For examples of the latter, see Chris Bailey, The Productivity Project: Accomplishing More by Managing Your Time, Attention, and Energy (New York: Crown Business, 2017); Matthew B. Crawford, The World beyond Your Head: On Becoming an Individual in an Age of Distraction (New York: Farrar, Straus and Giroux, 2016); Daniel Goleman, Focus: The Hidden Driver of Excellence (New York: HarperCollins, 2015); Neen James, Attention Pays: How to Drive Profitability, Productivity, and Accountability (Hoboken, NJ: Wiley, 2018).

2. Lorraine Daston and Peter Galison, Objectivity (Cambridge, MA: Zone Books, 2007).

3. Axel Volmar, ed. Zeitkritische Medien (Berlin: Kadmos, 2009); Wolfgang Ernst, Digital Memory and the Archive, ed. Jussi Parikka (Minneapolis: University of Minnesota Press, 2013).

4. Louis É. Javal, "Essai sur la phisiologie de la lecture," Annales d'Oculistique $80(1878): 240-274$.

5. August Ahrens, Die Bewegung der Augen beim Schreiben (Rostock, Germany: University of Rostock, 1891); Edmund B. Delabarre, "A Method of Recording Eye Movements," American Journal of Psychology 9, no. 4 (1898): 572-572; Edmund B. Huey, "Preliminary Experiments in the Physiology and Psychology of Reading," American Journal of Psychology 9, no. 4 (1898): 575-586; Edmund B. Huey, The Psychology and Pedagogy of Reading, with a Review of the History of Reading and Writing and of Methods, Texts, and Hygiene in Reading (New York: MacMillan, 1908); E. Rählmann, "Über den nystagmus und seine ätiologie," Archiv für Ophthalmologie 24 (1978): 237-242. 
6. Huey, "Preliminary Experiments in the Physiology and Psychology of Reading," 25-26.

7. Raymond Dodge and Thomas S. Cline, "The Angle Velocity of Eye Movements," Psychological Review 8, no. 2 (1901): 147.

8. Dodge and Cline, "The Angle Velocity of Eye Movements," 145.

9. $\quad$ Dodge and Cline, "The Angle Velocity of Eye Movements," 147.

10. Raymond Dodge, "Five Types of Eye Movement in the Horizontal Meridian Plane of the Field of Regard," American Journal of Physiology 8, no. 4 (1903): 307-329; Raymond Dodge, "The Participation of Eye Movements in the Visual Perception of Motion," The Psychological Review 11, no. 1 (1904): 1-14.

11. George M. Stratton, "Eye-Movements and the Aesthetics of Visual Form," Philosophische Studien 20 (1902): 343.

12. Stratton, "Symmetry, Linear Illusions and the Movements of the Eye," The Psychological Review 13, no. 2 (1906): 95.

13. Nicholas J. Wade, "Pioneers of Eye Movement Research," i-Perception 1, no. 2 (2010): 57 .

14. Guy Buswell, How People Look at Pictures: A Study of the Psychology and Perception in Art (Chicago: University of Chicago Press, 1935).

15. Buswell, How People Look at Pictures, 131.

16. Alfred L. Yarbus, Eye Movements and Vision, trans. Basil Haigh (New York: Plenum Press, 1967).

17. Benjamin W. Tatler, Nicholas J. Wade, Hoi Kwan, John M. Findlay, and Boris M. Velichkovsky, "Yarbus, Eye Movements, and vision," $i$-Perception 1, no. 1 (2010): 7-27.

18. Yarbus, Eye Movements and Vision, 192. In reference to the figure, Yarbus writes: "Seven records of eye movements by the same subject. Each record lasted 3 minutes. The subject examined the reproduction with both eyes. 1) Free examination of the picture. Before the subsequent recording sessions, the subject was asked to: 2 ) estimate the material circumstances of the family in the picture; 3 ) give the ages of the people; 4) surmise what the family had been doing before the arrival of the "unexpected visitor"; 5) remember the clothes worn by the people; 6 ) remember the position of the people and objects in the room; 7) estimate how long the "unexpected visitor" had been away from the family" (174).

19. Tatler et al., "Yarbus, Eye Movements, and Vision," 24-25.

20. Marianne DeAngelus and Jeff B. Pelz, "Top-down Control of Eye Movements: Yarbus Revisited," Visual Cognition 17, no. 6-7 (2009): 790-811.

21. Paul M. Fitts, "German Applied Psychology during World War II," American Psychologist 1 (1947): 151-161.

22. Fitts, "The Information Capacity of the Human Motor System in Controlling the Amplitude of Movement," Journal of Experimental Psychology 47, no. 6 (1954): 381-382.

23. Paul N. Edwards, The Closed World: Computers and the Politics of Discourse in Cold War America (Cambridge, MA: MIT Press, 1996). 
24. Ross Ashby, An Introduction to Cybernetics (London: Chapman \& Hall, 1956); Norbert Wiener, Cybernetics: or Control and Communication in the Animal and the Machine, 2nd ed. (Cambridge, MA: MIT Press, 1961).

25. Edwards, The Closed World, 179-180.

26. E.g. Donald E. Broadbent, "A Mechanical Model for Human Attention and Immediate Memory," Psychological Review 64, no. 3 (1957): 205-215.

27. Paul M. Fitts, Richard E. Jones, and John L. Milton, "Eye Movements of Aircraft Pilots during Instrument-Landing Approaches," Aeronautical Engineering Review 9, no. 2 (1950): 1-6.

28. Orit Halpern, Beautiful Data: A History of Vision and Reason since 1945 (Durham, NC: Duke University Press, 2015).

29. Arturo Rosenblueth, Norbert Wiener, and Julian Bigelow, "Behavior, Purpose and Teleology," Philosophy of Science 10, no. 1 (1943): 18-24.

30. Rosenblueth et al., "Behavior, Purpose and Teleology," 18.

31. Warren S. McCulloch, "Information in the Head," Synthese 9, no. 1 (1955): 235.

32. Maurice Merleau-Ponty, The Visible and the Invisible, trans. Alphonso Lingis, ed. Claude Lefort (Evanston, IL: Northwestern University Press, 1968).

33. Rudolf Arnheim, Visual Thinking (Berkeley and Los Angeles: University of California Press, 1969).

34. Fitts, "Information Capacity," 281.

35. Andrew T. Duchowski, Eye Tracking Methodology: Theory and Practice, 3 rd ed. (Cham, Switzerland: Springer International, 2017). Duchowski even describes the retina's photoreceptors as "transducers" (18).

36. Duchowski, ix, 3-4.

37. Wilson S. Geisler and Lawrence K. Cormack, "Models of Overt Attention," in The Oxford Handbook of Eye Movements, eds. Simon P. Libersedge, Iain Gilchrist, and Stefan Everling (New York: Oxford University Press, 2011), 439-454; Árni Kristjánsson, "The Intriguing Interactive Relationship between Visual Attention and Saccadic Eye Movements," in The Oxford Handbook of Eye Movements, 455-470.

38. Tobii Pro: Fields of Use," Tobii, accessed May 12, 2018, https://www.tobiipro. com/fields-of-use/.

39. Colin Milburn, Nanovision: Engineering the Future (Durham, NC: Duke University Press, 2008).

40. Simon Parkin, "Nvidia's Eye-Tracking Tech Could Revolutionize Virtual Reality," MIT Technology Review, July 21, 2016, https://www.technologyreview. $\mathrm{com} / \mathrm{s} / 601941 /$ nvidias-eye-tracking-tech-could-revolutionize-virtual-reality/.

41. Parkin, "Point, Click, and Fire in Virtual Reality—with Just Your Eyes," MIT Technology Review, June 23, 2015, https://www.technologyreview. $\mathrm{com} / \mathrm{s} / 538711 /$ point-click-and-fire-in-virtual-reality-with-just-your-eyes/.

42. Tom Simonite, "A Laptop That Knows Where You're Looking," MIT Technology Review, March 4, 2011, https://www.technologyreview.com/s/423198/alaptop-that-knows-where-youre-looking/. In fact, whole conventions have since been held with prototypes demonstrating the future of commands in screen-based media. See Simonite, "PC Makers Bet on Gaze, Gesture, Voice, 
and Touch," MIT Technology Review, January 10, 2013, https://www.technologyreview.com/s/509751/pc-makers-bet-on-gaze-gesture-voice-and-touch/.

43. Kyle Krafka, Aditya Khosla, Petr Kellnhofer, Harini Kannan, Suchendra Bhandarkar, Wojciech Matusik, and Antonio Torralba, "Eye Tracking for Everyone," arXiv preprint arXiv:1606.05814 (2016): 2176-2184.

44. Xucong Zhang, Yusuke Sugano, Mario Fritz, and Andreas Bulling, "Appearance-Based Gaze Estimation in the Wild," in Proceedings of the IEEE Conference on Computer Vision and Pattern Recognition (2015), 4511-4520.

45. Qiong Huang, Ashok Veeraraghavan, and Ashutosh Sabharwal, "TabletGaze: Unconstrained Appearance-Based Gaze Estimation in Mobile Tablets," Machine Vision and Applications 28, no. 5-6 (2017): 445-461; Yusuke Sugano, Yasuyuki Matsushita, and Yoichi Sato, "Learning-by-Synthesis for Appearance-Based 3D Gaze Estimation," in Proceedings of the IEEE Conference on Computer Vision and Pattern Recognition (2014): 1821-1828.

46. Krafka et al., "Eye Tracking for Everyone," 2.

47. Geoffrey Hinton, Oriol Vinyals, and Jeff Dean, "Distilling the Knowledge in a Neural Network," arXiv preprint arXiv:1503.02531 (2015): 1-9.

48. Sarah Sharma, In the Meantime: Temporality and Cultural Politics (Durham, NC: Duke University Press, 2014).

49. Arnheim, Visual Thinking, 13, 37.

50. Max Horkheimer and Theodor W. Adorno, Dialectic of Enlightenment, trans. Edmund Jephcott, ed. Gunzelin S. Noerr (Stanford, CA: Stanford University Press, 2007); William E. Connolly, Neuropolitics: Thinking, Culture, Speed (Minneapolis, MN: University of Minnesota Press, 2002).

51. Bernard Stiegler, Technics and Time, 1: The Fault of Epimetheus, trans. Richard Beardsworth and George Collins (Stanford, CA: Stanford University Press, 1998); Bernard Stiegler, Technics and Time, 2: Disorientation, trans. Stephen Barker (Stanford, CA: Stanford University Press, 2008); Bernard Stiegler, Technics and Time, 3: Cinematic Time and the Question of Malaise, trans. Stephen Barker (Stanford, CA: Stanford University Press, 2010).

52. Walter Benjamin, "On the Work of Art in the Age of Mechanical Reproduction," trans. Harry Zohn, in Illuminations, ed. Hannah Arendt (New York: Schocken, 1968), 217-253.

53. Richard H. Thaler and Cass R. Sunstein, Nudge: Improving Decisions about Health, Wealth, and Happiness (New York: Penguin, 2009).

\section{About the Author}

Alexander Monea is Assistant Professor of English and Cultural Studies at George Mason University (4400 University Drive MSN $3 \mathrm{E}_{4}$, Fairfax, VA 22030, United States). His research focuses on the history and cultural impact of big data and machine learning. His upcoming book examines heteronormative bias in internet content moderation. 


\title{
14. Ahead of Time: The Infrastructure of Amazon's Anticipatory Shipping Method
}

\author{
Eva-Maria Nyckel
}

\begin{abstract}
This text conducts a close reading of Amazon's 2013 patent for a "Method and System for Anticipatory Package Shipping" on three levels in order to investigate the patent's aspirations towards a potential hardwiring of temporality. First, through the lens of media theory, the patent is conceptualized as a medium for transporting knowledge over time itself. On a second level, the patent is framed as a logistical medium both due to its aspired effects in the logistical realm and its internal logic. Third, the specific form of anticipation, prediction and prophecy is investigated by leveraging Elena Esposito's understanding of (digital) prophecy with a particular focus on temporality.
\end{abstract}

Keywords: algorithms, Amazon, logistics, media theory, prediction, temporality

What if effects preceded causes and answers preceded questions? —John Durham Peters

In the industry of logistics, time is perhaps the most crucial resource not to be wasted. A variety of reference parameters are dedicated to measuring time in logistical processes, with names such as lead time, shipping time, and cycle time, as well as time bucket, time fence, time to market, and time of circulation. Reducing shipping latencies, providing real-time tracking of commodities, and forecasting consumer demand are elementary to structuring temporal

Volmar, A. and K. Stine (eds.), Media Infrastructures and the Politics of Digital Time: Essays on Hardwired Temporalities. Amsterdam: Amsterdam University Press, 2021 DOI 10.5117/9789463727426_CH14 
relations between customers, carriers, and merchants. Yet even within this context, Amazon's 2013 patent for a "Method and System for Anticipatory Package Shipping" marks a decisive shift in the industry's thinking on time saving in the way it extends logistics into the near-term future through a practice of prediction-based package shipping ahead of incoming orders. ${ }^{1}$

The investigation of anticipatory package shipping, also referred to in the patent text as a form of speculative shipping, is a speculative undertaking in itself: At this point, we cannot know how far Amazon has actually implemented the principles as described in the patent. The company has neither confirmed nor denied the implementation of the patented methods. ${ }^{2}$ However, one could assume that Amazon's logistical operations are coordinated in a similar way and that the patent provides at least fractional insights into how Amazon conducts logistical operations. ${ }^{3}$ On these grounds, I approach the patent from three media-theoretical perspectives with an eye to the system's potential implementation, analyzing the medium of the patent as a text genre with a specific temporality for the conveyance of knowledge, conceptualizing the anticipatory shipping method as a new form of logistical media for ordering time and space, and addressing the specific form of prediction and corresponding temporality of the patented methods in relation to Elena Esposito's analysis of prophecies in the digital age.

\section{The Patent as a Medium}

Patents, as the name would suggest, are rich sources for scholarly investigations in that they bring corporate research into the open. ${ }^{4}$ In the case of Amazon's anticipatory shipping patent, however, the text does not entirely deliver on this promise. Technical and methodical descriptions remain rather vague, and the patent text does not clearly designate the nature of the invention. As Albert Kümmel-Schnur has shown, it is not uncommon for companies to submit and be awarded patents where the material or process that is supposed to have been invented is unclear. ${ }^{5}$ This is the case with Amazon's patent for an anticipatory shipping process, which represents a bundle of methods, not a concrete technology. A close reading of the patent does not allow for an assessment of the concrete algorithms that are to be used or the parameters crucial for the predictions. What is described is rather a large number of different "embodiments," or potential material implementations, in an abstract way. The patent's vagueness raises a question of whether the company intentionally obfuscated corporate knowledge to secure economic advantage over potential competitors. But 
the language of patents in general might be a cause, being situated between engineering-speak and legalese.

The medial form of the patent, as Christian Kassung argues, is one that is necessarily situated between implicit and explicit knowledge. The act of publishing a patent is connected to making knowledge explicit, at least to some extent, that allows for the communication between the assignee-to-be of the patent and the patent-granting institution. ${ }^{6}$ At the same time, a patent gives room to discursive and diagrammatical operations and aims to establish a new order of knowledge. ${ }^{7}$ The degree to which the implicit knowledge of the inventor has to be made explicit is dependent on the possibility of effective communication between the patent-granting institution and the prospective assignee. ${ }^{8}$ Kassung argues, moreover, that patents never describe a bare technology or a concrete apparatus by itself but rather a collective of actants in Latour's sense that must be connected for the invention to be realized. ${ }^{9}$ Patents, in this understanding, project a future network of actants. Thus, patents themselves have an inherent temporal logic: they project a possible technological future that does yet not exist at the time of the patent's filing. ${ }^{10}$ Hence, patents are actors that open, close, concentrate, and diffuse technical stories at the same time. ${ }^{11}$

In the case of Amazon's anticipatory shipping patent, the company seeks to extend conventional logistics into the future through a new network of predictive actants. Central points or nodes of the logistical network of conventional package shipping are called hubs. A hub in the context of the patent can be a warehouse, fulfillment center, or sortation center. Fulfillment centers are in essence the e-commerce variation of what has traditionally been referred to as warehouses. ${ }^{12}$ Amazon's fulfillment centers are modelled on Walmart's distribution centers, which at their introduction took over the classical functions of warehouses. The change in name reflects the change in function for these structures; whereas a warehouse would store goods, in the ideal distribution center they are not stored at all. This is particularly evident with so-called cross-docking distribution centers, where incoming goods are immediately unpacked from trucks and sent through the center's conveyor belt system to the correct outgoing truck, headed for, say, a retail store. Amazon's fulfillment centers function somewhere in between a distribution center and a classical warehouse. ${ }^{13}$

The patent provides a description of conventional order fulfillment systems before elaborating the principles of anticipatory shipping, noting that, traditionally, an item "is not shipped ... until a customer places an order for that item to be delivered to a specific delivery address." ${ }^{14}$ In conventional logistics, a customer order initiates the shipping process, 
and the act of shipping triggers the payment. With anticipatory shipping, however, the company spends money on the shipping process without prior or simultaneous customer payment. Neither an order nor money is the input. ${ }^{15}$ This is particularly important given that Amazon would pay USPS and FedEx, or as of very recently, Amazon Delivery Service Partners, for transporting the package. ${ }^{16}$

In the patent a form of speculative shipping is introduced, or the "shipment of packages without completely specifying delivery addresses at the time of shipping. ${ }^{{ }^{117}}$ One important aspect of speculative shipping is the method of late-select addressing, or "late addressing," as it is also called in the patent, which occurs as a package passes through one of the hubs. This method allows for packages to be shipped without a specified delivery address and for the delivery address to be added en route after the package has shipped. A subform of speculative shipping is anticipatory shipping, which constitutes the patent's most interesting innovation:

In some embodiments, speculative shipping of a package may occur in anticipation of a customer ordering items in that package, but before such an order has actually occurred. In such embodiments, speculative shipping may also be referred to as anticipatory shipping. ${ }^{18}$

In other words, the patented methods facilitate the shipping of packages before they are even ordered, based on the ability "to forecast or predict customer demand for a given item."19 This forecasting model can ideally draw on large amounts of data about customers, such as previous wish list activities, shopping carts, product searches, and the duration of mouse hovers over items. ${ }^{20}$ What is predicted by this mechanism, in essence, are probable futures.

Of course, predictions can be wrong and orders might not be placed as expected. To minimize financial risk, the patent also accounts for false positives, or faulty predictions. What we might call a fallback mechanism can be set up to keep costs related to an already-shipped package as low as possible: redirecting the package to another geographical area where a future purchase is likely, returning the package to its point of origin, incentivizing a future purchase by offering a discount or other nudge toward a potential purchase, or considering the costs of an already-shipped package as an investment in customer's "goodwill":

In some instances, the package may be delivered to a potentially-interested customer as a gift rather than incurring the cost of returning or redirecting 
the package. For example, if a given customer is particularly valued (e.g., according to past ordering history, appealing demographic profile, etc.), delivering the package to the given customer as a promotional gift may be used to build goodwill. ${ }^{21}$

In this last option, false positives become a matter of building customer relationships. ${ }^{22}$ In the case of offering a discount and nudging potential customers toward a purchase, however, false positives from the anticipatory shipping algorithm are countered instead using Amazon's classic recommendation algorithm for curating the display of products. If a package were shipped but not yet met by a corresponding order, the product would be offered via the e-commerce platform to customers in near proximity to the current location of the shipped package at the given time. To make the offer more attractive, a discount could be provided.

Subsequent to determining the potential cost of returning or redirecting the near-proximity package, the package is offered to the potentially-interested customer at a discounted price, where the discounted price depends on the determined potential cost of return or redirection. ${ }^{23}$

Combining the anticipatory shipping and recommendation algorithms allows for the fulfilling of prophecies. A sale is not only predicted but also produced. ${ }^{24}$ In the following section, I discuss how this production occurs through a specific ordering of time, or a hardwiring of temporality.

\section{Anticipatory Shipping as New Form of Logistical Media}

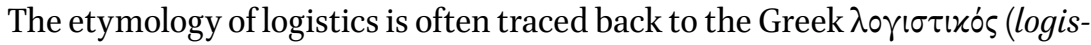
ticos), which means "skillful at calculation" but also "pertaining to reason." 5 In her work on logistics, Gabriele Schabacher (2008) refers to the French verb loger, originally meaning the temporary accommodation of soldiers or guests in a housing space. It was only this latter of three meanings of logistics that was taken up by researchers and experienced further differentiation in military-historical contexts. ${ }^{26}$ Logistics, from a current point of view, can be regarded as a discipline, a perspective, or a set of cultural techniques engaged in (re)ordering time and space. ${ }^{27}$ Monika Dommann argues that logistics, stemming from military strategies concerned with keeping fighting forces supplied, designates an engineering science dealing with the symbolic representation, monitoring, and control of flows of materials. 
The declared goal of managerial logistical thinking, as Schabacher (2008) describes it, is always optimization. Optimization is here understood in terms of permanent improvement of logistics management and organizational forms, aiming for the smooth flow of commodities, information, services, and persons, preferably in a cost-saving way. ${ }^{28}$ The just-in-time (JIT) model, also known as the Toyota Production System (TPS), concretely revolutionized logistical processes: time was considered one of the most valuable resources not to be wasted, with optimization being turned especially toward reducing wasted time. ${ }^{29}$

In recent years, shipping processes have accelerated markedly, with shipping latencies decreasing and JIT principles rendering commodities constantly in motion, requiring them to be at the right place at the right time and spending as little time as possible on shelves. The connection between the logistical paradigm and time can be seen clearly in the corporate quest for efficiency as timesaving. Deborah Cowen argues, with reference to total cost analysis, that prior to the logistics revolution, different business activities were seen as separate, with transportation being seen as following the production process and not as a moment of it..$^{30}$ With total cost analysis, this changed, resulting in a new hardwiring of both time and space in the realm of corporate logistics, as production facilities moved away from metropolitan areas and distribution centers took their place. Such relocations were counterintuitive within the old paradigm, but the higher transportation bills made sense in terms of lowering overall cost. ${ }^{11}$

Alongside Walmart, Amazon is one of the largest organizations operating in the realm of logistics and has built out a massive infrastructure for its operations. Attention to the infrastructures of contemporary networks of information and materials has been of great interest in recent media studies. "Infrastructuralism," as recently articulated by John Durham Peters, "suggests a way of understanding the work of media as fundamentally logistical." ${ }^{2}$ For our purposes, the insight can also be reversed: the work of logistics is fundamentally a question of media.

In his investigation of radar, Judd Ammon Case introduces logistical media as "media of orientation" that "have to do with order and arrangement first, and representation second, if at all." 33 This perspective on the power to arrange seems indeed useful for understanding anticipatory shipping. The arranging and re-arranging of relationships between different actors, such as retailers, carriers, and customers as well as goods, databases, and money, describes what Amazon does through different medial forms on a daily basis. The patented methods connected to anticipatory shipping are a way of rearranging the paths and in particular the temporal relationships of 
these people, things, and institutions. A particular infrastructural ordering of space and time produces a moment of exchange, a sale. It is only by having the particular material arrangement of fulfillment centers, trucks, planes, roads, sortation centers, and algorithms that the patent works. ${ }^{34}$

Peters describes logistical media in The Marvelous Clouds as having the job of "ordering fundamental terms and units" and setting "the terms in which everyone must operate." 35 As examples, he lists calendars, clocks, and towers, as well as names, indexes, addresses, archives, and money. Taking up the topic of new technologies later in the book, Peters adds that digital media, such as those we see in Amazon's order fulfillment systems, "serve more as logistical devices of tracking and orientation" and "revive ancient navigational functions: they point us in time and space, index our data, and keep us on the grid." ${ }^{36}$

Amazon's system of anticipatory package shipping is governed by an assemblage of digital media that produces an overlap of virtual and real space: the "real" space, inhabited by potential customers, is made manageable in terms of supply and calculation through a division into "geographical regions" where space is organized according to grids. ${ }^{37}$ The grid, as an instrument for partitioning, allocating, and relating entities, is a central feature of logistical media. Peters writes: "The job of logistical media is to organize and orient, to arrange people and property, often into grids. ${ }^{n}{ }^{8}$ The centrality of the grid to efforts at economizing time can be seen in Alan E. Branch's definition of logistics, from a business perspective, as the "time-related positioning of resources ensuring that material, people, operational capacity and information are in the right place at the right time in the right quantity and at the right quality and cost." 39 What Branch stresses, notably, is the "rightness" of place and time. Resources must be in the right place to be on time, and the more minutely structured are time and space, the more potential opportunity there is to be in a particular place on time. The ability to coordinate objects in a grid of space in real time, I argue, is made possible and provided through the digital—-thus, digital logistical media.

Real-time interconnection, as Ned Rossiter argues, is crucial to logistical optimization: "Without real-time interconnection logistical operations become exercises in inefficiency."40 Rossiter, in this way, and in contrast to Peters and Case, draws a line between prior forms of organization, such as clocks and calendars, and contemporary digital technologies. He describes logistical media as an "instrumentalization of location-aware mobile technologies" that are "designed to exert control over the mobility of labor, data, and commodities as they traverse urban, rural, atmospheric 
and oceanic spaces and traffic through the circuits of databases, mobile devices and algorithmic architectures. ${ }^{p 1}$

Without diminishing the non-digital entities involved in fulfillment centers, trucks, schedules, sortation hubs, planes, roads, railways, and container ports, I want to focus on the all-encompassing network of digital logistical media governing these entities. Amazon's patent describes a setting where predictive algorithms, which are inherently digital technologies, govern logistical processes. What is novel about the patent is a potential change in algorithmic logic rather than in any material shipping infrastructure. Just as total cost analysis comes to view business activities as interconnected, digital systems compose these activities as literally and technically interconnected. The alignment of data and goods described in Amazon's patent is both produced and ensured by digital devices of tracking and recording. In this regard, the text stresses the importance of "machine-readable identifiers." That is, inventory items and packages must be automatically traceable through "bar codes, magnetically or optically-readable characters, or other types of marking and scanning techniques," such as "radio frequency identifier (RFID) tags or other types of transponders. ${ }^{32}$ The technological identification of items allows for automatic control in the sense of both monitoring and governing their direction, i.e., routing.

Digital logistical media, as the example of Amazon's anticipatory shipping method makes clear, represent a culmination of logistical techniques into a potentially all-encompassing interconnective entity. In the contemporary situation in which our world is ubiquitously populated by digital computers, one could speak of a hardwiring of time (and space) through the networks of algorithms and digital devices that govern the flow of information and goods - thus setting the terms of a politics of digital time. The predictive logic described in the patent is leveraged for not only logistical operations but also customer behavior. The following section will therefore present a third perspective on the patent by investigating the specific logic of prediction inherent in the methods of anticipatory shipping.

\section{Anticipation, Prediction, Prophecy}

Predictive analytics have become increasingly important as processes of decision-making in various sectors of contemporary societies. ${ }^{43}$ The Latin praedictiō has two meanings as "the act of mentioning in advance," namely, "prediction" and "prophecy."44 Algorithmic predictions could be considered to carry out this second meaning as the prophecies of our time, in the way 
they structure the relation between present and future. Elena Esposito draws a striking comparison between ancient divinatory practices and today's prediction practices performed by “web intelligence." Prophecy takes on different meanings depending on whether the future is considered open or predetermined. In this regard, a key difference between divination and algorithmic prediction lies in each society's underlying understanding of the future. While divination speaks to an already-decided future, algorithmic prediction provides forecasts of probable futures. This difference becomes particularly relevant and visible in the case of false positives. If a divinatory prophecy did not come to pass, it was not the prophecy that was considered false. Because the future was regarded as predetermined and already decided, the interpretation was instead to blame: "one did not understand correctly, but the oracle was right." ${ }^{45}$ False positives were epistemologically impossible.

In a modern and contemporary understanding of time, the idea of a predetermined future has, in the same way, become epistemologically implausible. The future is rather considered as ever evolving, open, and developing from human actions: "it cannot be known in advance because it does not yet exist." ${ }^{46}$ What is possible, though, is the algorithmic forecasting of probable future behavior by looking at past behavior. When Esposito describes the effort of economically-oriented systems to bring future events and risks under control, she draws on Norbert Wiener's cybernetic notion of control. According to her reading of Wiener, "the past of a system is never sufficient to determine its future. One can collect all the possible information about the past, but it will not be enough to know the future."47

Even if we cannot know the future, predictions can be produced through algorithmic means. What we can get from algorithmic predictions, is a "statistical future," i.e., "the distribution of possible futures of the system (the present future)." ${ }^{48}$ This idea of multiple possible futures corresponds well to the patented methods of anticipatory shipping, which accounts in advance for multiple futures. The most peculiar aspect of prediction in anticipatory shipping is the way it accounts for false positives, what we called its fallback mechanism. This fallback mechanism is, again, the redirection of the package to another location, the return of the package to where it came from, or the production of consumer demand for the product by offering a discount and very short delivery time.

What remains unclear after a close reading of the patent, though, is what exactly would cause the fallback mechanism to take effect. Basically, the prediction would need to be declared wrong under specific circumstances. In the case of anticipatory shipping, one cannot know if a predicted purchase within a geographical area-after an indefinite amount of time-would 
have occurred after all. ${ }^{49}$ The validity of the prediction is temporally limited by, say, a "time to live" $5^{\circ}$ for the package - in order to set the fallback mechanism into place to - in case of embodiment c) - induce the sale. Of course, there is still no guarantee that the desired customer behavior will occur, but at least there is a second chance. The form of temporal control described in the patent follows the cybernetic meaning of control, as Esposito describes it:

Control, in this case, is achieved not by comparing the input (the present) with a goal (the future), but by comparing the input with memory (the past), in order to draw an always renewed and always open projection of possibilities. The future is rewritten again in each present. ... This future is open without being indeterminate, and uses the past to multiply the available possibilities. ${ }^{11}$

In the third embodiment of anticipatory shipping's fallback mechanism, reality is readjusted to the prediction. This embodiment reveals itself as the most radical articulation of rewiring temporalities because the future order within a geographical area is not only predicted but produced in the present. In other words, potentiality is sought to be turned into an actuality through technological, specifically, algorithmic, means.

\section{Conclusion}

The algorithmic governing of shipping networks and fulfillment centers towards anticipatory strategies represents an articulation of rewiring temporalities. With Amazon's predictive algorithms, it is not only time that is brought under digital control but also, and particularly, the aggregated purchase behavior of clients. As Wolfgang Ernst writes, "Algorithms do not focus on the individual event but detect patterns, rhythms, and regularities to be extrapolated into the future." $5^{2}$ The operation of nudging potential customers toward a purchase is basically an effort of aligning the "world" and prediction, of increasing the possibility that the prediction will come true.

In many cases in the press and online, Amazon's patenting of anticipatory package shipping has brought forth articulations of uneasiness. One potential reason for these reservations might be found in the company's lack of transparency regarding its algorithms. Robert Seyfert and Jonathan Roberge point out that users often expect Amazon's conventional recommendation algorithm to fail: 
The astonishment often expressed when Amazon's recommendation algorithms correctly predict (or produce) our taste, and directly result in a purchase, goes hand in hand with complaints of how wildly off the mark they are. We have come to expect failing algorithmic systems and we have indeed become accustomed to dealing with them. ${ }^{53}$

Indeed, following Seyfert and Roberge, at times the only response to Amazon's miscalculations is to laugh. It is remarkably hard to peek into the inner workings of algorithms and understand their potential mistakes, particularly involving the anticipatory package shipping methods on the table. To determine whether Amazon's algorithms succeed or not in predicting our aggregated taste and shopping behavior is basically impossible from the outside.

Not only do the predictive algorithms remain opaque, but the relations between patented and actually implemented methods at Amazon also remain unclear. Amazon has not yet commented on the implementation status of anticipatory shipping, as the company typically declines to comment on patents. ${ }^{54}$ A quick search for patents assigned to Amazon Technologies Inc. returns a list with 8,493 results. ${ }^{55}$ This might be an effect of the company's current policies, trying to patent everything that seems to be potentially applicable and profitable in the future. The long list of patents, however, makes it even more difficult to determine which patens have been or will be implemented.

Irrespective of the actual implementation status of the methods described in the anticipatory shipping patent, one can observe that Amazon seeks in its business practices to collect ever more customer data and offer ever shorter delivery times. Nevertheless, the patented methods can be used to think about the rewiring of temporalities set out by predictive algorithms in ever faster and more efficient logistical processes. In essence, the patent along with the presented approach, allows us to think about a rewiring of temporality in terms of effects preceding causes, or shipments preceding orders.

\section{Notes}

1. Joel R. Spiegel, Michael T. McKenna, Girish S. Lakshman, and Paul G. Nordstrom, Method and System for Anticipatory Package Shipping, US Patent No. 8,615,473, filed August 24, 2012, and issued December 24, 2013.

2. See Atle Mikkola Kjøsen, "Anticipating Realization: Value's Logic of Movement and Amazon's Anticipatory Shipping," Conference Paper, Historical 
Materialism (UK), 2014, https://www.academia.edu/9288107/Anticipating Realization_Values_Logic_of_Movement_and_Amazons_Anticipatory_Shipping1. After the patent on anticipatory shipping was published in late 2013, it got some attention from the news and various technology-oriented blogs, but there is only little scholarly work on this patent, notably that by Kjøsen. At this point, I would also like to sincerely thank Kjøsen for a productive email exchange and generous comments on the draft text. Furthermore, this paper owes a lot to and builds on the collaboration with Nikolaus Pöchhacker addressing the relation of anticipatory shipping to the production of markets, see Nikolaus Pöchhacker and Eva-Maria Nyckel, "Logistics of Probability: Anticipatory Shipping and the Production of Markets," in Explorations in Digital Cultures, ed. Marcus Burkhardt, Mary Shnayien and Katja Grashöfer (Meson Press, 2020), https://explorations.meson.press/ chapters/logistics-of-probability/.

3. For a close look on the influence of patents on software development in the US, see Gerardo Con Díaz, Software Rights: How Patent Law Transformed Software Development in America (New Haven, CT: Yale University Press, 2019); "Intangible Inventions: A History of Software Patenting in the United States, 1945-1985" Enterprise \& Society 18, no. 4 (2017), 784-794. doi:10.1017/ eso.2017.36.

4. An alternative take on patents would be their conceptualization as "imaginary media" themselves or as documents describing imaginary media, following Jussi Parikka's elaborations on Eric Kluitenberg (ed.), Book of Imaginary Media: Excavating the Dream of the Ultimate Communication Medium (Amsterdam and Rotterdam: Debalie and NAi Publishers, 2006). Given that patents are oriented toward future technology that may or may not be invented, or we may or may not know if a patent has been implemented as in the present case of Amazon's anticipatory package shipping, which could be described as "media imagined, non-existent, but worthy of exploration in terms of how it can reinvigorate current media cultural design and debates." Parikka, What Is Media Archaeology? (Cambridge: Polity Press, 2012), 61.

5. $\quad$ Albert Kümmel-Schnur, "Patente als Agenten von Mediengeschichte," in Bildtelegraphie: Eine Mediengeschichte in Patenten (1840-1930), ed. Albert Kümmel-Schnur and Christian Kassung (Bielefeld: Transcript, 2012), 16.

6. Christian Kassung, "Die Zukunft des Wissens und eine Geschichte der Patente," in Nicht Fisch - nicht Fleisch. Ordnungssysteme und ihre Störfälle, ed. Thomas Bäumler, Benjamin Bühler, and Stefan Rieger (Zürich: Diaphanes, 2011), 156. Although Kassung's text is mainly concerned with patents for concrete technical apparatuses, his diagnosis remains true for software patents and patented methods.

7. Kassung, "Die Zukunft des Wissens," 153. See Bowker (1992) as well as Pottage and Sherman (2010) For further analyses of patents, see Geoffrey Bowker, "What's in a Patent?," in Shaping Technology/Building Society: Studies in Sociotechnical Change, ed. Wiebe E. Bijker and John Law (Cambridge, MA, 
and London: The MIT Press, 1992), 53-74; Alain Pottage and Brad Sherman, Figures of Invention: A History of Modern Patent Law (Oxford: Oxford University Press, 2010).

8. Kassung, "Die Zukunft des Wissens," 156.

9. See for example Bruno Latour, Pandora's Hope: Essays on the Reality of Science Studies (Cambridge, MA: Harvard University Press, 1999).

10. Another reason why patents could be understood as documents describing imaginary media (see Parikka, What Is Media Archaeology, 61; Kluitenberg, Book of Imaginary Media).

11. Kümmel-Schnur, "Patente als Agenten von Mediengeschichte," 27.

12. See Kjøsen, "Capital and Post-Cybernetic Control: On Amazon's Patent for Anticipatory Package Shipping." Presentation on April 5, 2018, Faculty of Information and Media Studies, Western University, London, Ontario; Oliver Nachtwey and Philipp Staab, "Die Avantgarde des digitalen Kapitalismus," Mittelweg 36, no. 6 (2015), 67.

13. See Kjøsen, "Capital's Media: The Physical Conditions of Circulation" (PhD diss., University of Western Ontario, 2016), https://ir.lib.uwo.ca/etd/4156/.

14. Spiegel et al., 16.

15. Kjøsen, email message to author, July 11, 2018.

16. For more on Amazon Delivery Service Partner, see Louise Matsakis, "Why Amazon Is Giving Employees \$10,00o to Quit," Wired, May 14, 2019, https:// www.wired.com/story/amazon-delivery-paying-employees-to-quit/.

17. Spiegel et al., 16 .

18. Spiegel et al., 16. Emphasis added.

19. Spiegel et al., 17 .

20. Spiegel et al., 22 .

21. Spiegel et al., 22.

22. However, unasked-for gifts by corporations are not always the best idea in terms of Customer Relationship Management, as they basically violate the "rules" of gift-giving and can produce irritation, even anger, for customers. This was the case e.g. with Apple's "free gift" of automatically downloading U2's then-new album for all iTunes users in 2014. See Robert Booth, 'U2's Bono Issues Apology for Automatic Apple iTunes Album Download," The Guardian, October 15, 2014, https://www.theguardian.com/music/2014/ oct/15/u2-bono-issues-apology-for-apple-itunes-album-download.

23. Spiegel et al., 21.

24. At this point - on the important difference between demand and sale-I wish to quote Atle Kjøsen from an e-mail correspondence on July 11, 2018: "Often "demand" is confused for "need" and for "purchase." In the case of the patent, "demand" is actually already proven by Amazon because the consumer has added items to their wish list, shopping cart, looked at the items $\mathrm{x}$ number of times and so on. There is clearly a demand there, but that demand may not be backed up by cash. Hence, the whole point of logistics is to not match supply with demand, but match supply with cash. ... What is predicted and produced is not demand (it was already there), 
but a sale: the moment whereby commodities change hand for money. But in that case, it is not merely the algorithms that produce a sale, but also the physical infrastructure of FCs, hubs, trucks etc. (the infrastructure of the order fulfillment system). After all, if the package is not in a geographical area where demand has been detected, the sale cannot be produced."

25. The Brill Dictionary of Ancient Greek, 2015 ed., s.v. "logistics"; see also Deborah Cowen, The Deadly Life of Logistics: Mapping Violence in Global Trade (Minneapolis: University of Minnesota Press, 2014), 26; and Gabriele Schabacher, "Raum-Zeit-Regime: Logistikgeschichte als Wissenszirkulation zwischen Medien, Verkehr, und Ökonomie," Agenten und Agenturen: Archiv für Mediengeschichte, ed. Lorenz Engell, Joseph Vogl, and Bernhard Siegert (Weimar: Bauhaus University, 2008), 137.

26. Schabacher "Raum-Zeit-Regime," 137.

27. For more on cultural techniques, see Cornelia Vismann, "Kulturtechnik und Souveränität," Zeitschrift für Medien- und Kulturforschung 1 (2010), 171-181; Liam Young, "Cultural Techniques and Logistical Media: Tuning German and Anglo-American Media Studies," M/C Journal 18, no. 2 (2015), http:// journal.media-culture.org.au/index.php/mcjournal/article/viewArticle/961. For more on the ordering of time and space, see Monika Dommann, "Handling, Flowcharts, Logistik: Zur Wissensgeschichte und Materialkultur von Warenflüssen," Nach Feierabend 2011: Zirkulationen. Zürcher Jahrbuch für Wissensgeschichte 7, ed. David Gugerli et al. (Zürich: Diaphanes, 2011), 75. For a history of logistics from a media-theoretical perspective, see Schabacher, "Raum-Zeit-Regime" and Dommann, "Handling, Flowcharts, Logistik." For an investigation on the political economy and potential violence of global logistics processes, see Cowen, The Deadly Life of Logistics.

28. Schabacher "Raum-Zeit-Regime," 144f.

29. For more on TPS, see Taiichi Ohno, "Toyota Production System" (1978), The Roots of Logistics: A Reader of Classical Contributions to the History and Conceptual Foundations of the Science of Logistics, ed. Peter Klaus and Stefanie Müller (Heidelberg: Springer, 2012), 173-182.

30. Cowen, The Deadly Life of Logistics, 36-38. See also Kjøsen, Capital's Media, 78. Kjøsen argues for qualifying the current capitalist mode of production as "logistical ... due to the increased centrality of logistics to business since the 1970s" (73).

31. Cowen, The Deadly Life of Logistics, 38.

32. John Durham Peters, The Marvelous Clouds: Toward a Philosophy of Elemental Media (Chicago: University of Chicago Press, 2015), 37.

33. Judd Ammon Case, "Geometry of Empire: Radar as Logistical Medium" (PhD diss., University of Iowa, 2010), 1, http://ir.uiowa.edu/etd/474.

34. Kjøsen, email message to author, July 11, 2018.

35. Peters, The Marvelous Clouds, 37.

36. Peters, The Marvelous Clouds, 7.

37. Spiegel et al., 15. This real space is also built up and ordered by the infrastructure of fulfillment centers, sortation hubs and so on. These hubs are 
not placed randomly. Cowen elaborates on how logistics is a reorganization of space; before factories were located close to the market, but after the logistics started calculations of total cost, factories were often located closer to important transportation nodes.

38. Peters, The Marvelous Clouds, 37.

39. Alan E. Branch, Global Supply Chain Management and International Logistics (New York: Routledge, 2009), 1.

40. Ned Rossiter, "Coded Vanilla: Logistical Media and the Determination of Action," South Atlantic Quarterly 114, no. 1 (2015), 139.

41. Rossiter, Software, Infrastructure, Labor: A Media Theory of Logistical Nightmares (New York: Routledge, 2016), 8.

42. Spiegel et al., 17. See also Christoph Rosol, RFID. Vom Ursprung einer (all) gegenwärtigen Kulturtechnologie (Berlin: Kadmos, 2007).

43. See also Pöchhacker and Nyckel (2020), 1.

44. Oxford Latin Dictionary, 1994 ed., s.v., "praedictiō."

45. Elena Esposito, "Digital Prophecies and Web Intelligence," Privacy, Due Process and the Computional Turn: The Philosophy of Law Meets the Philosophy of Technology, ed. Mireille Hildebrandt and Katja de Vries (Oxon and New York: Routledge, 2013), 134. The future was perceived as predetermined, and therefore immutable. According to this belief, it could not be altered by human actions - "not even by those who try to oppose it" (134). As an example of the future's inevitability Esposito refers to the story of Oedipus.

46. Esposito, "Digital Prophecies and Web Intelligence," 134.

47. Esposito, The Future of Futures: The Time of Money in Financing and Society (Cheltenham: Edward Elgar, [2009] 2011), 194.

48. Esposito, The Future of Futures, 194; see also Wiener, Cybernetics: Or control and Communication in the Animal and the Machine (Cambridge, MA: MIT Press, 1948), 37, 92-93.

49. Alternatively or in addition, one could suspect that costs may play a decisive role in this scenario, though time and cost are, of course, connected quite closely. Thus, declaring a failed prediction could be something like "Possible sale $\mathrm{x}$ has a $\mathrm{y} \%$ chance in $\mathrm{z}$ hours/days." Kjøsen, email message to author, July 11, 2018.

50. "Time to live" (TTL) is an attributed number that enables a mechanism to prevent the indefinite rerouting of packages; however, in this case, data packets or datagrams in the Internet, enabled and structured by the Internet Protocol: "This number designates the maximum number of hops that that datagram is able to take before it is deleted. At each hop, the time-to-live is decreased by one. If the time-to-live reaches zero, the routing computer is obligated to delete the datagram. This ensures that datagrams will not hop around the network indefinitely, creating excess congestion." Alexander R. Galloway Protocol: How Control Exists after Decentralization (Cambridge, MA: MIT Press, 2004), 45. This description-respectively the reappropriation of the package metaphor as providing a surplus meaningworks surprisingly well for the temporal control of packages "hopping" 
through the network of hubs, fulfillment centers, and delivery centers as conceptualized in the anticipatory package shipping patent. For further discussion of this similarity between the routing of data packets and packages, see Christoph Neubert and Gabriele Schabacher, "Verkehrsgeschichte an der Schnittstelle von Technik, Kultur und Medien. Einleitung," in Verkehrsgeschichte und Kulturwissenschaft (Bielefeld: Transcript, 2012), 25.

51. Esposito, The Future of Futures, 194.

52. Wolfgang Ernst, Chronopoetics: The Temporal Being and Operativity of Technological Media, trans. Anthony Enns (London and New York: Rowman \& Littlefield, 2016).

53. Robert Seyfert and Jonathan Roberge, "What Are Algorithmic Cultures?" in Algorithmic Cultures: Essays on Meaning, Performance and New Technologies, ed. Seyfert and Roberge (London and New York: Routledge, 2016), $14 f$.

54. Alan Boyle, Amazon Wins a Pair of Patents for Wireless Wristbands That Track Warehouse Workers," GeekWire, January 30, 2018, https://www. geekwire.com/2018/amazon-wins-patents-wireless-wristbands-track-warehouse-workers/; Celan Yeginsu, "If Workers Slack Off, the Wristband Will Know (And Amazon Has a Patent for It)," New York Times, February 1, 2018, https://www.nytimes.com/2018/02/o1/technology/amazon-wristband-tracking-privacy.html.

55. Google Patents, searched June 14, 2019, https://patents.google.com.

\section{About the Author}

Eva-Maria Nyckel is a PhD candidate in media studies at the Humboldt University Berlin. Her research interests are logistical media, enterprise software and digital capitalism. In her PhD thesis she writes about the history, theory and politics of "programmable labour." 


\title{
15. Artificial Neural Networks, Postdigital Infrastructures and the Politics of Temporality
}

\author{
Andreas Sudmann
}

\begin{abstract}
This essay examines the infrastructures and temporalities of modern AI technology based on artificial neural networks (ANN) and aims to contribute to a more substantial understanding of its political challenges. In order to unlock the different temporalities of ANN, a theoretical framework for the relationship of media and infrastructures is suggested that also might help to distinguish between the different levels of analysis related to specific steps and aspects of the machine learning process (the collection and production of learning data, the training of AI models etc.). An important reference point for the following considerations is ethnographic research conducted at TwentyBN, ${ }^{1}$ a Toronto and Berlin based AI company specialized in ANN and computer vision that just recently developed an app for the fitness market.
\end{abstract}

Keywords: artificial intelligence, machine learning, artificial neural networks, computer vision, science and technology studies, media-ethnography

Since around 2012, machine learning based on artificial neural networks (ANN) has become the dominant paradigm of artificial intelligence (AI) research and increasingly determines the technological and infrastructural conditions of contemporary computer culture. Whether new developments in the field of text, speech, or image recognition, whether concrete use cases such as self-driving cars, virtual avatars, or medical diagnostic systems, the list of innovations that involves ANN-based machine learning is extremely

Volmar, A. and K. Stine (eds.), Media Infrastructures and the Politics of Digital Time: Essays on Hardwired Temporalities. Amsterdam: Amsterdam University Press, 2021 DOI 10.5117/9789463727426_CH15 
long. Although some scholars emphasize the epistemological limits of ANN, it is nevertheless surprising to see how, since very recently, they became such a powerful technology of prediction, capable of calculating and mastering the uncertainties and fuzziness of future events. ${ }^{2}$

The status of ANN as predictive systems already demonstrates the fundamental importance of investigating this technology in terms of its temporal dimensions. From the perspective of media studies, which in part also sees itself as a decidedly critical discipline, the particular relevance of this focus lies not only in exploring the epistemological potential of this specific approach in AI. Rather, it is the central aim of this essay to show how a deeper investigation of the infrastructures and temporalities of modern ANN-driven AI technology also contributes to a more substantial discussion of its political challenges.

Like basically all AI technologies since the 1950s, ANN have been negotiated mostly in the mode of utopian discourse, from Frank Rosenblatt's Perceptron model ${ }^{3}$ to Google's Neural Machine Translation. The term utopia, in the sense of its etymological origin, refers to a non-place, in temporal terms typically to the not-yet-existent of an unknown future. Indeed, the current advances in that particular field of AI do once again fuel the speculative notion of a so-called "technological singularity" as the very event after which all future development of machine intelligence will no longer be predictable or comprehensible to humans (while also signifying the very date when machines will be superior to humans in most, if not all, core areas of human expertise). ${ }^{4}$ Michel Serres has argued that the concept of time in itself articulates the dialectics of predictability and unpredictability. ${ }^{5}$ However, in the case of ANN, we are confronted with a specific dialectic or paradox of temporal orders that simultaneously resonates with the speculative dystopian (rather than eutopian) character connected to current visions of an artificial general intelligence: how the accelerated development of a predictive technology might turn into something — at least for humansfundamentally unpredictable and inaccessible.

But these characteristics are not concerned merely with future scenarios because ANN are already perceived fundamentally as black boxes not understandable or at least only partially understandable by humans. ${ }^{6}$ Their black-box status encompasses many aspects, not only the opacity of the ANN itself or its specific medial learning operations, or the tremendous speed of micro-temporalities connected to AI technologies and their respective decision-making processes that we can understand as submedial forms operating below the threshold of human perception, ${ }^{7}$ but also the complex industrial practices and environments that generate and form 
the technological processes of modern AI. In other words, the event of a technical singularity is an escalation of what is already being discussed as one of the core problems of ANN technology: its fundamental inaccessibility. Obviously, such an event (if it ever becomes a reality) will result in enormous distortions, not least in political terms. Against this very background, one must take such an event as seriously as possible, even if, from today's perspective, it seems a scenario of a still distant future or unlikely in principle. Nevertheless, it is not very helpful to constantly speculate about the political or social consequences of a technology's future if we have just started to understand the current situation of AI in general and ANN in particular, both theoretically and historically. Even if the present and its temporality is always exactly what is already over when we talk about it. ${ }^{8}$ Accordingly, the aim of this essay is to explore the relation between infrastructures and temporalities with regard to ANN-based machine learning, specifically to provide a substantial basis for future political discussions of this technology.

A central aspect of ANN in political terms, particularly concerning its present form, is that we are dealing with a powerful technology of optimization and automation, which in numerous areas not only expands the respective capabilities of humans, but also clearly outperforms them. And it is worth highlighting that such ANN systems do not just master domain-specific tasks faster or more efficiently than humans. Instead, in many areas, such as image recognition, they also outperform humans in qualitative sense. However, as many scholars working in the transdisciplinary field of science and technology studies have argued, it is problematic to limit the political-epistemological relationship to the binary relationship of humans and machines or humans and technology. While it is important to stress that current machine learning technologies are still very much dependent on the activities of human beings and human labor, we also have to acknowledge that we are dealing with complex infrastructures and chains of operations, where humans, things, practices, and media are inseparably connected.

\section{Theoretical Approaches to Media and Infrastructures}

But how do we make sense of these different layers of temporalities involved in the industrial and scientific infrastructures of current AI? And how deep do we have to explore all the elements that constitute and shape these smart technologies? The answer to this question can only be given if we are willing to investigate the specifics of ANN technologies and try to understand the complexity of the technology in terms of its manifold 
temporal dimensions, at least on a conceptual level. Obviously, there is no lack of potentially useful approaches: Scholars working in the field of media archaeology, science and technology studies, historical epistemology, critical code studies, platforms studies, etc., have provided many accounts for the critical understanding of our data-driven algorithmic culture, also and especially with regard to the nexus of infrastructures, media, and temporality. ${ }^{9}$ And yet we have to acknowledge that studies in this specific area of research (as with media studies) have neglected machine learning in general and ANN in particular as an important field of research for a very long time. In the course of the current AI boom and especially since the spectacular victory of DeepMind's AlphaGo, the situation has changed significantly: AI has already become the prevalent topic in every field of the humanities and sciences. Nevertheless, it has to be said that the debate on ANN or machine learning in fields such as media studies, and cultural studies more generally, is still in its infancy. But what does it mean to approach the infrastructures and temporalities of AI and ANN, especially from a media studies perspective? And even more central in theoretical terms, what is the difference between infrastructures and media (if there is any) as key concepts for understanding AI technologies?

As one might imagine, there is not easy answer to this question. And yet, while infrastructures and media share certain characteristics-for example, that they refer to the cultural productivity of (partly) invisible operations and structure $^{10}$ - I would claim that a key difference between the terms is that "infrastructure" is a concept more useful for describing the arrangement or organization of (different) media, while, in turn, media are the heterogenous entities that constitute and contribute to shaping certain infrastructures in the first place. This consideration is by no means at odds with the idea that an infrastructural or logistic dimension is central to many diverse forms of "old" and "new" media. ${ }^{11}$ Still, we need a term for the arrangement and organization of different media, especially with regard to systemic and rule-based aspects of their stabilization, as well as with regard to the interoperability and connectivity of and associated with media and their related practices. And it seems to me that the term infrastructure is suitable for precisely this purpose.

In the following, I would like to demonstrate how the distinction between media and infrastructure is also helpful for understanding the different temporal logics and dimensions of AI technologies: For example, although the differences between media as input data play an important role in choosing and shaping a particular AI infrastructure for mastering an individual learning task, we also have to take into account that media differences can be decisive for developing advanced forms of AI. For this very reason, it is 
important to deal with the specifics of the relationship between media and infrastructures as they pertain to AI technologies.

However, as I outline in this chapter, ANN systems are characterized by a rather general temporal characteristic that is of great relevance both transhistorically and across different applications and specific infrastructures, and as such challenges the perception of ANN as a recent manifestation of digital change.

An important reference point for the following observations and considerations is my current ethnographic research at TwentyBN, ${ }^{12}$ a Toronto and Berlin based AI company specialized in Deep Learning solutions and computer vision that just recently developed an app for the fitness market.

\section{Big Data, Machine Learning, Crowdworkers, and the Politics of Temporality}

To unlock the different temporalities of modern ANN and specifically to understand the political dimension of their media and infrastructures, we first of all have to distinguish between different levels of analysis related to specific steps and aspects of the machine learning process. One important perspective in this regard is to shed light on the level of collecting and producing the large amounts of data necessary for training a machine to master a certain learning task. For example, in the case of so-called supervised machine learning, it is not enough simply to have access to large data sets; it is also necessary to have pairs of corresponding inputs and outputs as training data. A profound challenge here is that in many use cases, learning data in accurately labeled form is not available, and producing it can be a very time-consuming and costly working process. To address this problem, AI companies like TwentyBN specializing in ANN technologies hire legions of crowdworkers through platforms such as Amazon Mechanical Turk or CrowdFlower, responsible for labeling and producing the learning data. A general and and economically productive advantage of crowdsourcing in temporal terms is that instead of having a few employees working on monotonous, repetitive tasks again and again, such activities can be distributed more or less in parallel to hundreds or thousands of workers, thus saving a massive amount of time. ${ }^{13}$

One of the downsides of this form of work in late capitalism is that crowdworkers are typically poorly paid, which in turn regularly has a negative impact on the work they are engaged for. For their labor to pay off at least to some extent, they must complete their specific tasks as quickly 
as possible. Hence, to fulfill the task of producing and labeling data most efficiently, it happens quite often that they develop subversive strategies to earn more money within a certain time frame. Unsurprisingly, such practices of "cheating" do have serious consequences; in essence, it means that the algorithms are trained with corrupted data. As a result, AI companies like TwentyBN usually cannot use the data produced by crowdworkers directly; instead, they are forced to monitor and evaluate its quality using test algorithms or manual inspection. As the activities of crowdworkers illustrate, ANN systems and their specific infrastructures do have profound effects on the temporalities of lived experience, they not only exert pressures of conformity or standardization but also lead to strategies for avoiding the temporal regimes of a cognitive capitalism and its infrastructures now increasingly shaped by data-driven machine learning.

The creation of appropriate learning data can take months or even years, depending on the specific purpose of the learning data and AI model. The training and testing of an AI model is also very time-consuming. For high-end AI applications, such as advanced machine vision systems, training involves not only thousands but typically even millions of such cycles or epochs, while other less complex classification tasks, such as distinguishing simple geometrical forms (as in Rosenblatt's perceptron model), might only demand a few hundred training cycles. Hence, the temporal span of a training process can also vary a lot, from a few hours to several weeks. More generally, how fast an ANN can be trained for a certain problem depends on number of different parameters, including the quantity and quality of the training data, the specific architecture of the ANN, and the hardware resources available. For these very reasons, it is crucial to understand the specific characteristics of AI infrastructures that make use of ANN and other technologies of machine learning.

\section{Real-Time Capability and the Temporal Challenges of Cognitive Capitalism}

Another important point to consider regarding the temporal dimensions of AI infrastructures is that many intelligent systems must be capable of operating in real-time.

When for example TwentyBN recently developed its fitness app, it was particularly important to the company that their AI system is capable of providing immediate feedback to the users whenever they perform a certain exercise like jumping jacks or push-ups incorrectly. 
And also especially in sensible application areas, such as self-driving cars, ANN-based AI models involve strict latency requirements and "demand lightning-fast deep learning inference, usually within tens of milseconds for each sample."'14

Many modern AI technologies are therefore media configurations, which should or must guarantee a certain degree of responsiveness (Rechtzeitigkeit), which means they have to operate with regard to a varying ratio of processing speeds and a time window to be adhered to by these processes. ${ }^{15}$

While forms of "inference acceleration" have significantly improved over the last few years, also due to new hardware architectures like Google's TPUs, there is a further serious problem of temporality to consider here. For example, ANN models (like any other machine learning model) are based on the assumption that there are no "distributional shifts in the input and output data over time. ${ }^{{ }^{16}}$ Machine learning and in particular ANN systems work so well, regardless of the specific prediction task, because the world, i.e., the structure of data a statistical model of AI can learn, usually does not change quickly or with hard transitions but usually slowly and smoothly. ${ }^{17}$ And yet, the important assumption of ANN and other machine learning models that the distribution of input and output data remains more or less stable over time does have its specific limits, especially if we think about application areas like information security, "where fast-paced evolution of the underlying data generating mechanism is a norm (in the case of security, it is because both players, the defender and the adversary, are constantly striving to outmatch his opponent by changing his own strategies, thus exploiting the opponent's unguarded vulnerabilities)." ${ }^{m 8}$

Nevertheless, the basic principle of the industrial use of modern technology as a whole is to ensure that technical problems can be dealt with quickly and flexibly, for example, in the field of AI with regard to the much-discussed problems of bias structures. However, this does not always work as smoothly as desired. When in 2016 Microsoft presented its chatbot Tay to the public, the company had to shut down the system only after sixteen hours. Users quickly hacked the system by training it for racist, anti-Semitic, and sexist articulations, which unsurprisingly led to quite a large public controversy. But even if the example of Tay illustrates some serious problems and limits of current machine learning systems, we should not forget that at least with regard to machines, problematic bias structures can be easily corrected, or at least there is the option to simply shut down systems that do not work properly. And this is obviously different with regard to human beings.

However, within current academic discourses of philosophy, social science, and cultural studies, temporal aspects seem to play a less important 
role when we discuss the specific potentials of AI. This may be because AI itself is overdetermined by a rhetoric of progress emphasizing efficiency and flexibility in accordance with the neoliberal logic of the temporal regimes of late capitalism.

While this form of subjugation to neoliberal orders of time is typically rightly questioned by scholars across different fields, in the humanities and beyond, we should also keep an eye on the potentials of AI, even if the technology and its temporal order as a whole or in part are never neutral, serve neoliberal interests, or take on a questionable and teleological logic of progress. To give just one of many examples, if traffic will become more and more dominated by autonomous vehicles in the future, or if road traffic will be regulated by modern AI systems, this may be seen as a problematic affirmation of neoliberal values of optimization and efficiency because the intention is not simply to reduce traffic jams but also to create the possibility of coping with greater traffic loads, which in turn represents a serious ecological problem. On the other hand, there is at least some evidence that self-driving cars will significantly reduce the frequency of traffic accidents, simply because they are faster and better able than humans to recognize dangerous situations as such. ${ }^{19} \mathrm{~A}$ critique of $\mathrm{AI}$, which in particular focuses on aspects of temporality, should therefore take such ambivalences of technology into account and discuss them in a differentiated way.

\section{The Potential of ANN to Understand and Identify the "New"}

In any case, it is interesting to note that the critique of AI often includes a critique of the temporality of these systems. For example, the fact that machine learning technology inevitably uses past data to predict the future has often led to skepticism from scholars, claiming that data-driven machine learning systems based on ANN are inherently conservative and incapable of producing something genuinely new. ${ }^{20}$ Indeed, one could argue that recent discussions about algorithmic biases underpin this critical perspective. Likewise, many of us know from our everyday experience that it can be quite boring when, for example, recommender systems simply suggest titles similar to the music we already like. It might be also fair to say that we don't necessarily have to make the algorithms responsible for this: If we have the impression that an AI-powered system is only suggesting music we already know or like, we could also take this as an invitation to question the limitations of our taste in music. But that's not the point I'm primarily concerned with. Instead, I would like to question the criticism I have just presented for two other reasons. 
First, doubts seem justified as to whether ANN-based systems are really incapable of producing something new in the emphatic sense. Isn't the example of DeepMind's AlphaGo a powerful reminder that we can witness an AI model operating with moves that are perceived as innovative and surprising by the global community of Go players? Of course, prima facie it seems legitimate to argue that, according to such a perspective, one does not just take the rhetoric and PR strategies of the AI industry for granted, but also carry out a questionable anthropomorphizing and mystification of technical systems that in their core consists of statistical or computational processes. Nevertheless, it might be advisable to distinguish between Genesis and Geltung here. Similar to Turing's argumentation in the 1950 s regarding the justification of his famous test, one could argue that what matters is less whether an AI system really is creative or if it de facto has the potential in itself to create something genuinely new. Rather, what counts is if the system manages to appear to be creative or innovative.

Second, the question would be if the fetishization of AI, which has often been criticized, does not provoke another form of fetishization, namely the fetishization of an anthropocentric figure of the emphatically new. ${ }^{21}$ All "human" achievements, whether in art or science, are in fact based on complex and preceding mediators, i.e., on already existing technologies, media, infrastructures, communication processes, and forms of knowledge. Obviously, we apply different standards to machines than to people. But why? Do we have to face the fact that a post-anthropocentric view of AI is so difficult to accept, not only because we are not willing but also because we are simply unable to do so? I do not suggest we have to answer that question in the affirmative. Rather, the point is that one must be aware of the specific paradoxes when discussing anthropocentric concepts such as learning, knowledge, or the "new" in relation to intelligent machines.

However, the key challenges that AI systems are currently confronted with are indeed less about being able to identify or generate something completely new than about being able to take account of the temporal dimension as well as the specific context of data.

\section{Recurrent Neural Networks and the Temporal Significance of Media Differences}

It is important to acknowledge that also in this very area ANN have made significant progress in the last ten years. A crucial factor here was the advanced development of so-called recurrent neural networks (RNN). A 
key characteristic of RNN is their ability to identify patterns in sequences of data, e.g., for learning tasks such as text understanding, speech recognition, and even stock market developments. To do so, RNN need to fulfill the same task for each of the sequence's elements, while the output depends not only on the current input but also on the previous computations. In other words, RNN have a kind of memory function (internal state) that allows information to persist. Theoretically, RNN can process data sequences of infinite length, but in practice they are constrained so that they can look back only a few steps. Although the gradients of RNN can quite easily be computed, they are difficult to train due to their nonlinear iterative characteristics. According to Ilya Sutskever,

A small change to an iterative process can compound and result in very large effects many iterations later; this is known colloquially as "the butterfly-effect." The implication is that in an RNN, the derivative of the loss function at one time can be exponentially large with respect to the hidden activations at a much earlier time. Thus, the loss function is very sensitive to small changes, so it becomes effectively discontinuous. ${ }^{22}$

However, these limitations and difficulties do not change the fact that the leaps in performance in the area of speech recognition, such as with Alexa, and Siri, or in the field of machine translation have been considerable since around 2009.

Recurrent neural networks have also made significant progress in the field of computer vision. In 2016, I had the honor of introducing what might be considered as the most important ANN innovation since the famous ImageNet-Paper by Krizeshvsky et al $^{23}$ in an article for the German newspaper Frankfurter Allgemeine Zeitung, ${ }^{24}$ where I described how for the first time in the history of information technology, TwentyBN developed an ANN system to automatically recognize hand gestures and basic (and yet complex) activities (even before Baidu, Facebook, and Google were able to master this fundamental AI problem). And, indeed, the infrastructure (or the media) that enabled this computer vision technology could hardly be more relevant from a media studies perspective: For the first time and in contrast to most approaches in this field of AI research up to this point (which typically used photographs or still images for computer vision tasks), TwentyBN's model has been trained with hundreds of thousands of short video clips, showing different kinds of (basic) activities, such as opening objects, throwing and catching something, or stack things. ${ }^{25}$ The implications of this approach for our thinking about infrastructures, media, temporality, 
and their relations are very significant: What the system developed by TwentyBN implicitly demonstrates is that already the sheer media difference between time-based and non-time-based media seems to be an essential precondition for the development of an advanced AI. Accordingly, since 2017, we are confronted with new epistemological condition of our global computer culture, namely that we witness the emergence of an AI that is already capable of understanding basic forms of common-sense knowledge, i.e., perceiving and "understanding" the world in ways similar to human beings. ${ }^{26}$

And - hardly less relevant—we also have to consider that the specific technological potential of media difference to allow such advanced forms of machine intelligence exists - at least in a certain sense-outside the realm of history, culture, and ideology. On the one hand, the specific content and meaning of the videos shown to the system is less decisive for the learning operation than the basic fact that it is trained with moving images at all. On the other hand, the "content" of the model matters insofar as the system has to be trained with a great variety of gestures and actions so that it is capable of generalizing well. Nevertheless, the learning system also perceives and processes every input similarly, without a deeper sense of meaning, the gesture of a Hitler salute (Hitlergru $\beta$ ) not being different from the activity of turning a bottle, stacking books, or doing jumping jacks. Hence, ANN-based computer vision takes place within an infrastructural arrangement in which the broader cultural, social, and historical contexts of the learning material play no significant role in terms of their technological operations. Functionally decisive is the diversity of the displayed material in itself, not its specific and semantically charged composition. ${ }^{27}$

As the example outlined above shows, it is very important to focus on the specific, inherent temporalities of learning algorithms, their Eigenzeitlichkeit, which at least partially retain autonomy and contingency over those temporal regimes that characterize the commercial and scientific infrastructures of machine learning technologies as a whole-such as the inscription of history or the specific temporal logic of how machine learning tasks are organized as industrial or scientific processes.

Against this background, in view of their principal indifference to content and meaning, ANN systems are a media technology that is not fundamentally different from the gramophone or photography as technologies of the nineteenth century or from the digital computer as a technology of the twentieth century. And yet ANN turn the time relations of information technology upside down. 


\section{Massive Parallelism as a Postdigital Configuration of Future Information Technology}

An important prerequisite for the implementation of a model such as that developed by TwentyBN, however, is the performance of the modern computer hardware on which the networks are trained, namely graphics processing units (GPUs) ${ }^{28}$ What distinguishes the temporality of ANN both generally and transhistorically is their massive parallelism. At the end of the 1980s, Friedrich Kittler wrote:

Today's artificial intelligences run faster, more parallel, but not fundamentally different from those who "follow the principle of the Universal Discrete Machine ... With it, the media system is closed. Storage and transmission media both merge into a principle circuit that can simulate all other information machines simply because it stores, transmits and calculates in each individual program loop. ${ }^{29}$

In contrast to Kittler's perspective, I would like to argue that ANN systems, especially because of the parallelism of their information processing, do indeed represent a significant break with the logic of digital computers of the von Neumann architecture and that they actually stand for the emergence of a post-digital computer culture avant la letter. ${ }^{30}$ In fact, neural networks, whether artificial or "natural," are in at least two respects a counter-model to the way digital computers function according to von Neumann's serially organized architecture. First, since a single artificial neuron is usually either active or not, in this respect it usually functions according to binary logic, such as the switching states of a digital computer. The weighting of the activity between the neurons, i.e., the strength of their connections, is however mostly represented by floating point numbers (positive and negative) in neural networks. And this representation is so finely structured that the corresponding values can be understood as quasi-analog. As media of information transmission, ANN do not operate using binary units, such as 0 and 1 , but in an almost analog form (even if the analog values are still based on a digital substrate).

Second, it must be emphasized that the masses of interconnected neurons, activated by an input, fire together simultaneously or in parallel, thus ultimately forming a complex emergent system that abolishes the discreetness of the elements it consists of (the layers of neurons and their connection). This extreme or massive parallelism of information processing can indeed count as the essential characteristic of ANN, distinguishing it from the von 
Neumann architecture of classical digital computers. Due to the described properties, an ANN is therefore a blurred system (Unschärfesystem) with probabilistic results, whose operations can be described rather as analog than digital..$^{11}$ Whether ANN is primarily implemented as software, as it is at present, or increasingly as hardware, as it may be in the future, is irrelevant for its characterization as a post-digital information technology. Whatever future processor technologies modeled on neural networks will look like concretely, they share the basic characteristics that already position ANN as a "software medium." Anyone who simply understands the current developments of ANN, particularly in terms of its political dimensions, as a further expression of the digital revolution or the digitization of culture is in fact using the wrong category - at least in part.

\section{Conclusion}

In recent decades, the evolution of computers and the processes of digitization have been extensively investigated with regard to their temporal conditions, implications, and effects. Again it was Friedrich Kittler who argued that the computer, as a universal medium that can scan and simulate all other media, is in principle the end of media history..$^{32}$ Furthermore, several scholars have conceptualized the temporal logics of digitization as a transformation process of simultanization. The current renaissance of ANN now once again draws attention to the history of sub-symbolic information processing and its specific logics of temporality. With a few notable exceptions, the field of media and cultural studies has until very recently payed little attention to this. Paul Virilio, for example, has written about AI-driven vision machines that replace human perception, machines that look back at us and observe us, but despite its interest in the dromological view on temporality, he did not realize the enormous temporal implications of the connectionist AI paradigm that is now on the rise again. ${ }^{33}$

But what exactly has changed? One could assume that thanks to the massive parallelism of ANN-based machine learning we are confronted with a new dimension or quality of technological acceleration and thus have reason to believe that the event of a technological singularity might indeed not be that far away. But this remains to be seen. Obviously, the era of digital computers has not yet ended. Nevertheless, it is important to think about the changed conditions of a sub-symbolic computer culture, which we may no longer be able to adequately describe as digital. 
In contrast to the world of cultural imaginations, where immortality and death, series and events, infinity and finiteness, constitute a symbolic, interwoven network and thus a mysterious world sui generis, the question of AI infrastructures and their temporalities as a techno-political condition of the present almost adheres to something soothingly down-to-earth: be it as a view of the material preconditions of metaphysical speculations or as a more precise determination of the organizational relationship of media in relation to their environments. Perhaps today more than ever, to preserve the utopian potential of AI technology, we must lead it out of the mode of its speculative discourse.

\section{Notes}

1. "Media and Infrastructures of Artificial Intelligence: Computer Vision, Transfer Learning, and Artificial Neural Networks as Black Box.", funded by the German Research Foundation since 2019.

2. See Ian Goodfellow, Yoshua Bengio, and Aaron Courville, Deep Learning (Cambridge, MA: MIT Press, 2015).

3. See Frank Rosenblatt, "The Perceptron: A Probabilistic Model for Information Storage and Organization in the Brain," Psychological Review 65, no. 6 (1958): 386-408. https://doi.org/10.1037/hoo42519.

4. See Vernor Vinge, "The Coming Technological Singularity: How to Survive in the Post-Human Era," in NASA Lewis Research Center, Vision 21: Interdisciplinary Science and Engineering in the Era of Cyberspace, NASA Conference Publication CP-10129 (San Diego, CA: NASA, 1993), 11-22; Nick Bostrom, Superintelligence: Paths, Dangers, Strategies (Oxford: Oxford University Press, 2014).

5. See Michel Serres and Bruno Latour, Conversations on Science, Culture, and Time, trans. Roxanne Lapidus (Ann Arbor: University of Michigan Press, 1995).

6. Andreas Sudmann, "Deep Learning als dokumentarische Praxis," Sprache und Literatur 48, no. 2 (2017): 155-170.

7. Wolfgang Ernst, Digital Memory and the Archive, ed. Jussi Parikka (Minneapolis: University of Minnesota Press, 2013); Mark B. N. Hansen, Feed-forward: On the Future of Twenty-First-Century Media (Chicago: University of Chicago Press, 2015); and Shintaro Miyazaki, "Algorhythmics: Understanding Micro-Temporality in Computational Cultures," Computational Culture 2 (2012), http://computationalculture.net/algorhythmics-understanding-micro-temporality-in-computational-cultures/.

8. Jacques Derrida, Marges de la Philosophie (Paris: Minuit, 1972).

9. See Lisa Parks and Nicole Starosielski, eds., Signal Traffic: Critical Studies of Media Infrastructures (Urbana: University of Illinois Press, 2015); Gabri- 
ele Schabacher, "Medium Infrastruktur: Trajektorien soziotechnischer Netzwerke in der ANT," Zeitschrift für Medien- und Kulturforschung 2 (2013): 129-148; Axel Volmar, ed., Zeitkritische Medien (Berlin: Kadmos, 2009).

10. Susan Leigh Star, "The Ethnography of Infrastructure," American Behavioral Scientist 43, no. 3 (1999): 380.

11. See John Durham Peters, The Marvelous Clouds: Toward a Philosophy of Elemental Media (Chicago: University of Chicago Press, 2015).

12. "Media and Infrastructures of Artificial Intelligence: Computer Vision, Transfer Learning, and Artificial Neural Networks as Black Box," funded by the German Research Foundation since 2019.

13. Nevertheless, as I have learned during my ethnographic study so far, there are many crowdworkers who take on these microtasks repeatedly, sometimes even for years.

14. Yanchen Wang, "Deep Learning in Real Time-Inference Acceleration and Continuous Training." Medium.com, 2017. https://medium.com/syncedreview/deep-learning-in-real-time-inference-acceleration-and-continuous-training-17dac9438bob.

15. Uwe Brinkschulte and Heinz Wörn, Echtzeitsysteme. Grundlagen, Funktionsweisen, Anwendungen (Berlin: Springer, 2005); Volmar, Zeitkritische Medien, 10,18 .

16. Wang, "Deep Learning in Real Time."

17. Ethem Alpaydin, Machine Learning. The New AI (Cambridge, MA: MIT Press, 2016).

18. Wang, "Deep Learning in Real Time."

19. See Sprenger in this volume.

20. See, e.g., Matteo Pasquinelli, "Machines that Morph Logic: Neural Networks and the Distorted Automation of Intelligence as Statistical Inference," Glass Bead Journal, Site 1, Logic Gate: The Politics of the Artifactual Mind (2017), https://www.glass-bead.org/article/machines-that-morph-logic/?lang=enview.

21. See also Marvin Minsky's remarks from the early 1980 os on the creativity of machines and how in comparison human creativity tends to be overrated and mystified whenever people discuss the limits of AI. Marvin Minsky, "Why People Think Computers Can't," AI Magazine 3, no. 6 (1982), 3-15.

22. Ilya Sutskever, "Training Recurrent Neural Networks." PhD diss., University of Toronto, 2003, 10-11.

23. Alex Krizhevsky, Ilya Sutskever, and Geoffrey E Hinton, "ImageNet Classification with Deep Convolutional Neural Networks," Advances in Neural Information Processing Systems 25 (2012): 1097-1105.

24. Andreas Sudmann, "Wenn die Maschinen mit der Sprache spielen," Frankfurter Allgemeine Zeitung, November 2, 2016. However, a first demo version was not presented to the public until 2017. See https://www.youtube.com/ watch $? \mathrm{v}=7$ Fntm $8 \mathrm{cCbNM} \& \mathrm{t}=45$ s.

25. Later, in early summer 2019, TwentyBN started to develop its AI trainer for the fitness market. Accordingly, the company then focused on creating 
videos, showing different fitness exercises in order to train the AI model. The corresponding app called "Fitness Ally" was released exclusively on iOS in May 2020.

26. At the same, we have to acknowledge that AI's learning systems, even if they most certainly become more and more embodied as well as situated in our empirical world, nevertheless always remain an alien form of intelligence, very much different from the embodied intelligence of human beings.

27. Of course, this erasure of context is again a problematic ideological operation. In addition, the operations of the learning algorithms and in particular the learning data itself can be biased in many different respects. See Alexander Monea, "Race and Computer Vision," The Democratization of Artificial Intelligence: Net Politics in the Era of Learning Algorithms, ed. Andreas Sudmann (Bielefeld: Transcript, 2019), 189-208.

28. Sudmann, "Wenn die Maschinen mit der Sprache spielen."

29. Friedrich Kittler, "Die Künstliche Intelligenz des Weltkriegs: Alan Turing," in Friedrich Kittler and Georg-Christoph Tholen, ed., Arsenale der Seele. Literatur- und Medienanalyse seit 1870 (Munich: Fink, 1989), 187-202, 195.

30. Andreas Sudmann, "Szenarien des Postdigitalen. Deep Learning als MedienRevolution," in Machine Learning: Medien, Infrastrukturen und Technologien der Künstlichen Intelligenz, ed. Christoph Engemann und Andreas Sudmann, (Bielefeld: Transcript, 2018), 55-73.

31. At the same time, it is still important to note that ANN based systems operate as deterministic machines. See Sudmann, "Szenarien des Postdigitalen."

32. Friedrich Kittler, Grammophon, Film, Typewriter. (Berlin: Brinkmann und Bose, 1986).

33. Paul Virilio, La Machine de Vision (Paris: Galilée, 1988).

\section{About the Author}

Andreas Sudmann is a media scholar and AI researcher at Ruhr-University of Bochum, Germany. He is the author of several books, edited collections, and essays in the field of media and digital culture studies, including The Democratization of Artificial Intelligence. Net Politics in the Era of Learning Algorithms (Columbia University Press/ Transcript 2019), "Games and Artificial Intelligence" (Special Issue of Eludamos. Journal for Computer Game Culture Vol. 10 (2019), and "Artifıcial Intelligences" ("Künstliche Intelligenzen"), German Journal for Media Studies (Zeitschrift für Medienwissenschaft) Vol. 21 (2019). 


\title{
16. Technics of Time: Values in Future Internet Development
}

\author{
Britt S. Paris
}

\begin{abstract}
To understand how time can be considered both a technical and sociocultural design value, this chapter investigates named data networking (NDN), a new networking protocol conceptualized to replace addressed-based internet protocol and promises to increase both the speed and the efficiency of the internet. Bernard Stiegler's technics and time framework guides the analysis of time-based values articulated by the NDN project principals to demonstrate how collective temporality is built into technical systems as engineers reconcile social and cultural concepts of temporality with computational and architectural time and resource constraints in network design. This chapter shows that although efficiency is a timebased technical value driving NDN development, the sociocultural values of information temporality is much less understood by project principals.
\end{abstract}

Keywords: technics, internet infrastructure, time, temporality, technology design, Bernard Stiegler

The advent of ${ }_{5} \mathrm{G}$, Google Fiber, and other recent technologies that augment internet speed has meant very little to most people. With the technologies currently available to most users, this super high-speed internet is difficult to access. ${ }^{1}$ At the same time, internet service providers (ISPs) underserve large swathes of the population because they are averse to building the new infrastructure that would bring internet services up to speed for these groups. Internet speed is addressed in this paper as a fundamental dimension of technical time present in technological engineering projects. To characterize how time is considered both a technical and social design value in developing internet infrastructure, I look to named data networking (NDN),

Volmar, A. and K. Stine (eds.), Media Infrastructures and the Politics of Digital Time: Essays on Hardwired Temporalities. Amsterdam: Amsterdam University Press, 2021 DOI 10.5117/9789463727426_CH16 
a new networking protocol conceptualized to replace addressed-based internet protocol and, according to its project documents, dramatically increase both the speed and the efficiency of the internet.

The internet is often described as a "network of networks" that transmits information, or data, in the form of small, discretized packets, using a standardized set of instructions for communication procedures, called protocols. Packets then circulate through the internet following the procedures of TCP/IP (Transmission Control Protocol/Internet Protocol), HTTP (Hypertext Transfer Protocol), and other common protocols that structure internet networks. Alex Galloway's book Protocol points to the ways these structuring procedures effect patterns of control over human relation after decentralization. ${ }^{2}$ He states that protocols promote information speed, so that networked computer-disseminated information becomes a "natural extension of the user's own body."

Picking up where Galloway leaves off, I elaborate in this chapter on a specific example of how protocols interact with and extend users' bodies to promote information speed. Guiding this project is Bernard Stiegler's notion of technics as fundamental to the phenomenon of time. For Stiegler, technics are co-constitutive of human temporality, where technics refers broadly to material practices, techniques, and technologies that shape and are shaped by human temporality and the time of social coordination. In the first volume of Technics and Time, Stiegler focuses on subjective temporality, analyzing how technologies of materialized, externalized time inform how individuals experience and conceive of time. ${ }^{4}$ Extending this analysis in volumes two and three, he addresses collective temporality through the modes by which time has been made material and exterior to the human and, importantly for this chapter, how this externalization of time allows for social and cultural coordination. ${ }^{5}$ Applying Stiegler's framework to the time-based values articulated by the NDN project principals, we can see how notions of collective temporality are built into the technical systems surrounding protocols and how engineers reconcile social concepts of time with computational and architectural constraints in network design. We also see, however, the current limits of these engineering promises and how, although efficiency is a timebased technical value driving NDN development, the user-facing, social value of information temporality is much less understood by project principals.

\section{Internet Past, Present, and Future}

Internet Protocol (IP) is an elaborate addressing system that determines the origins and destinations of packets. Transmission Control Protocol 
(TCP) sets parameters for sending and confirming receipt of packets as they are transported to and from their destinations. TCP requires end-to-end communication, or that a sender and receiver at each end must confirm they are both online for a packet to be sent. The TCP/IP layers connect the lower-level hardware and the user-facing applications layers and are often called the networking and control layers using the International Organization for Standardization (ISO) framework. ${ }^{6}$ Once two networks are connected, end-to-end communication becomes possible with TCP/ IP. In this scenario, any end node on the internet can communicate with any other, regardless of their physical location or network affiliation. TCP/ IP's end-to-end communication requires only that the end nodes "know" traffic transmitted through the network, therefore keeping the network architecture itself "dumb" or unaware of any details about the packets being transferred. It is this end-to-end design that many proponents claim has allowed the internet the openness to become a global communication system. ${ }^{7}$

The widespread popularity of social media and mobile devices in the mid-20oos precipitated a paradigm shift in the nature of internet traffic. Mobile phones liberated people from their desktops and pushed for increased internet connectivity to accommodate users who tapped into mobile streams on the go. While the original premise of the Web was sharing existing documents, social media encouraged a notion of a new mode of using the Web that generates and transmits enormous amounts of data. The increased demand for connectivity and application-based communication caused internet researchers and funding agencies to worry that the new paradigm shift would be the demise of TCP/IP and end-to-end communication. ${ }^{8}$ To this end, the National Science Foundation's (NSF) Computer and Information Science and Engineering (CISE) program initiated the Future Internet Design (FIND) program, the first-generation future internet projects funded by the NSF. 9 In 2009, the NSF hosted a Future Internet Summit to survey the results and formulate a call for the next round of projects under the banner of Future Internet Architectures (FIA). For this round of funding, sociologists and policy experts were mobilized under the banner of the Values in Design (VID) Council to help formulate the call and to work with the FIA Architecture project winners as part of an anticipatory ethics project. This project pushed FIA engineers to begin the design process with socio-cultural values in mind..$^{10}$ In 2010 , CISE funded four projects at $\$ 8$ million each for three years; in 2014, Named Data Networking (NDN) was one of three programs that were awarded a further three years of Next Phase support through 2017. ${ }^{11}$ 


\section{Named Data Networking (NDN)}

Fundamentally, NDN wants to increase internet efficiency by simplifying networking to transmit data based on the name of the data, not its location. Overall, the NDN documents show six architectural principles that guide the NDN architecture. The first three, they say, are gleaned from the successes of IP routing, and the latter three build from the failures or challenges that IP routing has presented in recent years. Nearly every document concerned with what NDN is and how it works describes the hourglass architecture of the existing TCP/IP internet that "makes the original IP design elegant and powerful" because it takes packaged data and delivers it to applications and users. The hourglass architecture with the narrow waist is represented in figure 16.1. It centers on a "universal network layer (IP) implementing the minimal functionality necessary for global interconnectivity." ${ }^{\prime 2}$

The thin waist of named data is the focus of NDN design. This design feature is opposed to the thin waist of IP architecture that transmits packets according to IP addresses. This simple change at the thin waist results in significant differences between IP and NDN in their function.

In IP networks, nodes and links may overload once content becomes popular and is requested often, such as a video going viral. In NDN, more requests also mean more nodes will have a copy of the popular content in the cache. ${ }^{13} \mathrm{NDN}$ 's routing strategy then focuses on the IP address and control signaling to transmit packets, but it instead focuses on requests or "interests" calling for named data cached within the network nodes. The probability that a node near the application or a user on the path to the content generator has a cached copy of the content increases by its popularity. Via the NDN caching mechanism, copies of content are automatically distributed toward the parts of the network where the request is made. ${ }^{14}$ One will note, however, that push notifications from producers of interests are not something that NDN is built to accommodate because all data must be requested. This makes it difficult to build real-time applications over NDN, for reasons detailed later in this chapter.

Moreover, the popular press promotes NDN as an architecture that is "faster" or "swifter," or that "allows smoother content streaming" than today's TCP/IP-based internet. ${ }^{15}$ Given that these various terms for improved efficiency are lauded in the project documents as advantages and that they form the public-facing rationale for the projects, it makes sense to examine what these time-laden terms mean in practice. 


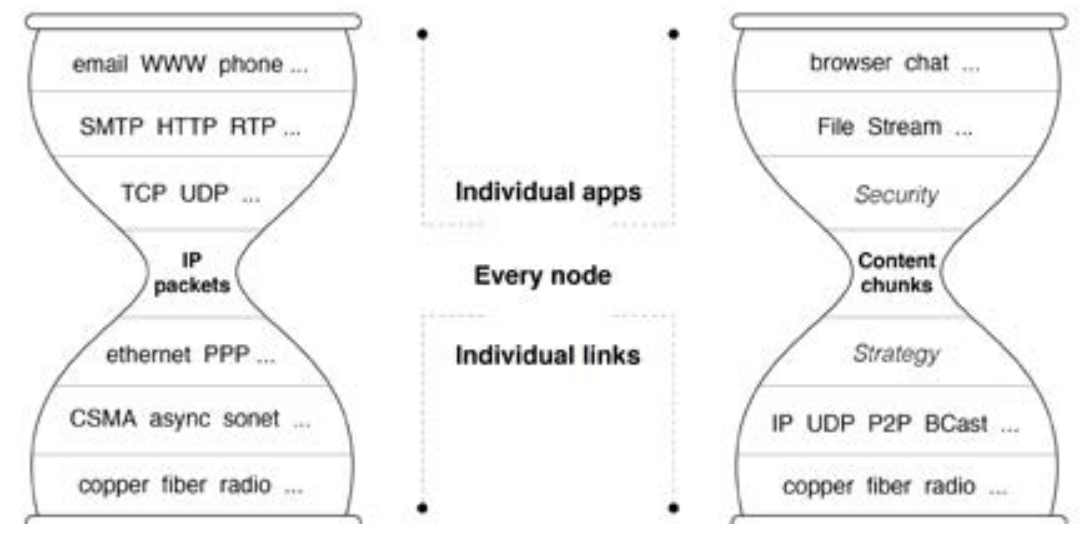

Figure 16.1. Comparing IP and NDN at the narrow waist ${ }^{16}$

\section{Efficiency as Technical Value}

Stiegler's first volume of his Technics and Time series focuses on how all technologies, broadly defined, are both material and temporal, capable of physical movement across space and memory transmission across time. In keeping with Stiegler's notion of technologies as material and, as such, temporal artifacts, the builders of the NDN technical system make decisions about time and how to conceive of it.

In the design of technical systems, the broad notion of efficiency is often cited as the primary goal. It is also a clear juncture at which the material dimensions of technical time become bound with notions of collective temporality. The importance of time as one of the first and most fundamental computational resources was highlighted by Ada Lovelace in 1843 in her description of the technical requirements for Charles Babbage's Difference Engine:

In almost every computation a great variety of arrangements for the succession of the processes is possible, and various considerations must influence the selections amongst them for the purposes of a calculating engine. One essential object is to choose that arrangement which shall tend to reduce to a minimum the time necessary for completing the calculation. ${ }^{17}$

Efficiency is bound with time and is articulated as a primary goal of networking research. This sentiment was reflected in a focus on efficiency in many of the NDN documents. Shilton found that with NDN, efficiency was 
often articulated as dynamism, in comparative statements such as, "Content transfer via [NDN] is always secure, yet the results show that it matches the performance of unsecured HTTP and substantially outperforms secure HTTPS." ${ }^{18}$

Lixia Zhang, NDN's primary networking engineer, suggested that efficiency drives internet technology and that user demand determines how engineers balance efficiency with functionality, but she noted that user-facing designs only move forward as technology allows them to do so. ${ }^{19}$ Indeed, the most frequently expressed values in the NDN founding documents were those responding to technical pressures, specifically methods for ensuring improved efficiency within the material constraints of the network architecture. And, moreover, many of the values emphasized by the NSF's 2007 request for proposals were technical values such as scalability and reliability. ${ }^{20}$ This is not surprising in a research setting where technical innovation is the primary motivator and marker of success. However, when taken together, these expressions of values suggest that the sociocultural values that the VID Council hoped to impart in the anticipatory ethics stage of the project were understood as less important than the technical values.

Zhang's remarks also encapsulate the model by which NDN network engineers think about values in design. User demand is understood superficially in terms of the technological functionality at the application layer, instead of any complex reckoning of what social values, hierarchies, and other ethical considerations might actually affect user activities, broadly defined. Other NDN respondents articulated notions of promoting efficiency by balancing computational resources like bandwidth and storage; the simplest computational resources are those of computation time (the number of steps necessary to solve a problem) and memory space (the amount of storage needed while solving the problem).

NDN's lone application developer noted that when producing applications that can generate and handle real-time audiovisual streams, there are issues of timestamps, which are fundamental to the coordination of nearly all networks. Routing strategies, such as the best-route strategy used in the TCP/IP internet requires data to be timestamped to be made into packets and sent. Similarly, NDN's routing strategy mentioned above does the same. However, because data must be called by name in real-time flows, each piece of data must have a timestamp or the order number available in the data's namespace. As each segment is produced, the consumer must call for the data using the proper name, order, or timestamp. Then the application must order the data incoming to the consumer in the right way and quickly enough so that it appears to be a real-time stream on the consumer side. 
At the level of applications, Gusev describes that the overhead for the packet header for the NDN named data alone is like $30 \%$ of its size, which is really inefficient." ${ }^{21}$ Gusev reported that when developing applications: "We work on algorithms first and make sure they are configured properly. Once that is sorted, we are very concerned with how well the algorithms work in a particular scenario. Generally, we aren't as interested in optimization as we are in just getting the applications to work.,"22

This highlights two important issues that must be mentioned in terms of NDN and new internet infrastructure engineering in general: First, infrastructure rarely faces users, so it perhaps makes sense that they aren't thinking as much about speed or user-facing temporality, even though this is lauded in public-facing documents. Second, building out infrastructure is slow, difficult work. Gusev, working alone, struggles with designing even basic applications because the NDN namespace, a core component of the NDN architectural design, is not configured to facilitate real-time applications.

While managing efficiency is overwhelmingly considered to be a balancing act between time and the materiality of the technical system, and users are not considered in the equation, there are technical junctures at which the materiality of the system is subject to the temporalities of social coordination. Gusev noted that NDN uses $\mathrm{C}++$ code for all application development because that language has low barriers to entry and allows new collaborators to focus on the important issues of technical design instead of learning a new language. In this case, using $\mathrm{C}++$ saves everyone time and assumes that the optimization of the work will happen at some later date. Similarly, protocols by their very common definition entail sociocultural coordination. NDN testbed manager John DeHart maintained that Network Time Protocol (NTP) is instrumental in running the testbed. ${ }^{23}$ NTP coincides with IP and is an enduring standard that has been established by standards governance bodies. NTP syncs devices within networks according to time-stamped data to ensure smooth end-to-end communication over the internet networks. The in-project decisions to use a common programming language and run the testbed in NTP suggest an aim toward easier in-project coordination, so that the work is also interoperable with other standardized bits of infrastructure.

Thinking through how notions of efficiency are leveraged and put into practice in the NDN project shows how time is considered a material thing - a computational resource that can be broken into many subsets, assigned timestamps and organized accordingly in technical practice. This technoscientific truth is alluded to in volumes two and three of Stiegler's 
Technics and Time, with reference to how networks transmit temporal objects quickly and widely on the basis of informatics:

Informatics, as Daumas understood it, is the industrial exploitation of information's value made possible by the development of electronic technologies as elements of the mastery of speed through the stabilizing, processing, and transmission of signals that are recordable and storable in electronic memory, making possible the control of information's circulation though the establishing of networks. ${ }^{24}$

For Stiegler, the result of this new programming culture industry amounts to the "pauperization" of the social and cultural realms; however, he leaves open to further analysis how this happens in practice. Indeed, he seems to regard informatics as monolithic and incapable of being questioned or held accountable, and treats humans as mere agents within the informatics industry who do the work of pushing technology and demanding higher speeds of humans. What becomes necessary is to bracket out the part of Stiegler's project that focuses on the cultural implications of informatics and investigate how the technological project in question, NDN, is constructed by people to push toward interface speed and consider how NDN engineers think of notions of speed in their work.

\section{Speed and User-Facing Temporality}

The notion of speed permeates Stiegler's Technics and Time. Following its definitions throughout the series, one can get lost in a number of complex anthropological examples that point to the human possibilities of memory and information transmission across space and how contemporary technologies accelerate society and culture to the degree that the present becomes ungraspable and the future nearly incomprehensible. The definition I use for this project is that of the acceleration of technology, which Stiegler claims urges humans to move ever faster and faster to keep up with increasingly "real-time" technical systems. ${ }^{25}$ He locates Adorno and Horkheimer's analysis of the culture industry within the longer arc of information technology, seeing a connection between today's data practices and the way cinema historically encouraged a loss of social and communal interaction in favor of industrial prerogatives for sustaining increases in production and economic profit through regulating consumption. ${ }^{26}$ He says the informatics culture industry demands higher speeds but says little of the values animating the 
industry other than, in naming it a culture industry, tying it to capitalism's push to render all of human life as a commodity. Although technological design practices that value material efficiency account for part of the equation of technical drivers of human time, they cannot complete it. It becomes necessary to address human factors, in this case, by unraveling these concepts of speed and how they manifest—or don't—in the articulated culture and political views of the NDN project principals.

At face value, NDN's engineers envision their specific protocols not so much as affecting the user-facing speed of applications but as offering, each in its own way, a new palette of possibilities at the application layer because of how protocol decreases network latency or enables higher performance at the network edges. In the project with NDN, the principals alluded to, but never clearly articulated, the idea that speed relates to user-facing temporal experience. Moreover, the ways the principals articulated speed are particularly evocative of how they view the advantages of their respective projects. However, engineers have a hard time effectively building for protocol and interface speed in their work.

Jeff Burke noted that, while NDN is just a networking solution that seeks to improve efficiency in the transmission of data over the internet, it is possible that some notion of faster user experience features might be possible:

NDN is not going to change the speed of light. It's not going to change the typical behavior of networks. Is it going to be the same for the end user? I'm not sure. For example, maybe the idea of scrubbing video that's streaming, because of the way that it happens on NDN versus how it typically happens on IP-something like random access into video may actually perform better. While you're not talking about fundamentally the network, I'd say the application-level behavior might be different. ... If we're interested in applications that do more real-time selections of-everything from the perspective on a scene from an immersive camera or light-field camera-anything that involves making quick decisions about what's being delivered over the network. ${ }^{27}$

Increasing efficiency is again a persistent topic in engineers' articulations of time as a resource or user-facing temporality in these projects. User-facing speed, on the other hand, is more of a countervailing concept that forces technologists to think about temporality as the social value of their work.

Speed, or, more broadly and colloquially, user-facing temporality, is a social dimension of efficiency, a crucial concept in NDN engineering that has many potential valences. In technical practice, smaller-scale latency 
management in system components makes user-facing performance or speed possible. Furthermore, locating speed as a subcategory of efficiency highlights how dogged adherence to the technical design value of efficiency seeks to make the design appear frictionless to users and, as a byproduct, the technical underpinnings of the system inscrutable.

\section{Materiality and Cultural Coordination of Technical Time}

Recalling Alex Galloway's claim that protocols promote information speed, so that the information purveyed through computational devices become a "natural extension of the user's own body," the moments of NDN design presented in this chapter support the idea that networking protocols materialize time into information in a way that is beyond the perceptual capacities of humans. ${ }^{28}$ While the principals seem to be unclear or even neglectful of how this new protocol schema would change user-facing temporal experience at the interface, there is evidence that the NDN technical system would conceivably allow different types of temporal experiences. At the same time, NDN invokes human temporal perception and experience as a partial justification for its work.

Time is materialized into information as packets and data are named, time stamped, and transported in order as they move through the NDN network, as, for example, in Gusev's discussion of the way timestamps are allocated and used in the development of applications that can handle real-time audiovisual streams. Through these temporal codes, time is made into a thing and used to determine the most efficient path for the data to take. This most-efficient path not only entails the lowest resource cost to the network but also, as a result of its low overhead, theoretically causes the network to function faster.

In-project standards, such as the use of $\mathrm{C}++$ code, facilitate the development of applications because of their low overhead and lowered use of resources in the network and in any user's CPU. Preexisting protocols such as NTP also impart material requirements (the need for time-stamped data) in the process of design and as such represent fundamental obstacles or technical concerns that must be attended to in the design processes of ordering functions in technical systems.

The technologies that Stiegler and, before him, Lewis Mumford wrote about are defined as material, whether the physical objects that mediated time in Stiegler's case or those that performed social or cultural mediations in Mumford's case. In the same way, new networking protocols are not only 
grounded in but also concerned with materiality. The engineers' technical goals - primarily, but not exclusively, efficiency—structured every choice they made. The analysis presented here indicates that the technical design value of efficiency is chiefly a material concern, that is, one of maximizing use of material computational resources and keeping latency low, so that the system can perform faster.

Finally, in NDN, we see that technical materiality has directly caused problems with the social coordination of these protocol projects. While efficiency and a faster internet were NDN's partial stated goals, building out the network and troubleshooting has taken more time than they had originally imagined. Principals from NDN articulated how the namespace load carried with each piece of data or content project is still quite bulky, as it takes up more physical space and resources to process, and contributes to the material load of these technologies. Thus, the social and presumably economic good of the speed touted by NDN that would come from optimization has not yet come to pass.

Much work still must be done to get the NDNs' functional efficiency to be on par with the conceptual efficiency touted by project principals. It remains to be seen whether this imagined efficiency or any combination of the related concepts discussed in this chapter will come to fruition for NDN. In any case, we see how efficiency and its subset of speed are used as marketing tools to make an argument about NDN's viability for the uninitiated. The notion of speed is bound with concepts of the user-facing temporality that would be made possible by using NDN instead of IP. However, this short meditation has shown that NDN engineers are not thinking very much about users, nor social or cultural values; they are instead more concerned with the materiality of time as it is measured and distributed through the NDN technical system. At present, NDN is built with regard to speed in name only. I suggest that a deeper engagement with values would require the principals to think seriously at all stages in the process to think about how future interfaces will be built on top of the new networking protocol, how those interfaces will affect users, their subjectivity, their bodies, and their relationship to collectivity. 
Notes

Some language, analysis, and interview data presented here are also found in the author's previous work: Britt Paris, "Time Constructs, The Origins of A Future Internet” (University of California, Los Angeles, 2018).

1. Farhad Manjoo, "What Do You Do With the World's Fastest Internet Service?" Slate, March 12, 2013, http://www.slate.com/articles/technology/ technology/2013/03/google_fiber_review_nobody_knows_what_to_do_ with_the_world_s_fastest_internet.html; and “Today, Kansas City. Tomorrow, Oklahoma City!" Slate, March 13, 2013, http://www.slate.com/articles/ technology/technology/2013/o3/google_fiber_internet_service_after_kansas_city_where_will_the_gigabit_network.html.

2. Alexander R. Galloway, Protocol: How Control Exists after Decentralization (Cambridge, MA: MIT Press, 2004), 8.

3. $\quad$ Galloway, Protocol, 67.

4. $\quad$ Bernard Stiegler, Technics and Time, 1: The Fault of Epimetheus, trans. Richard Beardsworth and George Collins (Stanford, CA: Stanford University Press, 1998).

5. Stiegler, Technics and Time, 2: Disorientation, trans. Stephen Barker (Stanford, CA: Stanford University Press, 2009); Technics and Time, 3: Cinematic Time and the Question of Malaise, trans. Stephen Barker (Palo Alto, CA: Stanford University Press).

6. Hubert Zimmerman, "OSI Reference Model-The ISO Model of Architecture for Open Systems Interconnection," IEEE Transactions on Communications 28, no. 4 (1980): 425-32, https://ieeexplore.ieee.org/stamp/stamp.jsp?arnumber $=1094702$.

7. David D. Clark, "The Design Philosophy of the DARPA Internet Protocols," Proceedings of SIGCOMM '88, Computer Communication Review 18, no. 4, 106-114, https://doi.org/10.1145/52324.52336; David D. Clark, John Wroclawski, Karen R. Sollins, and Robert Braden, "Tussle in Cyberspace: Defining Tomorrow's Internet," IEEE/ACM Transactions on Networking 13, no. 3 (June 2005), 462-475, https://doi.org/10.1109/TNET.2005.850224; J. H. Saltzer, D. P. Reed, and D. D. Clark, "End-to-End Arguments in System Design," ACM Transactions on Computer Systems 2 no. 4 (November 1984), 277-288. https://doi. org/10.1145/357401.357402; Tarleton Gillespie, "Engineering a Principle: 'Endto-End' in the Design of the Internet," Social Studies of Science 36, no. 3 (2006): 427-57, https://doi.org/10.1177/0306312706056047. Mark A. Lemley and Lawrence Lessig, "The End of End-to-End: Preserving the Architecture of the Internet in the Broadband Era," SSRN Scholarly Paper ID 247737 (Rochester, NY: Social Science Research Network), http://papers.ssrn.com/abstract=247737.

8. Clark et al., "Tussle in Cyberspace”; Gillespie, "Engineering a Principle."

9. Darleen Fisher, "US National Science Foundation and the Future Internet Design," in ACM SIGCOMM Computer Communication Review 37 (2007): 85-87, http://www.nets-find.net/InvitationToJoin.php; "NSF Future Internet 
Architecture Project," National Science Foundation Future Internet Architecture Project (n.d.), http://www.nets-fia.net/.

10. Helen Nissenbaum, Luke Stark, and Katie Zeiwitz, "Values in Design Council: An End of Project Report NSF Eager: Values in Design in the Future Internet Architecture," 2013, http://www.nyu.edu/projects/nissenbaum/vid/ pdfs/VIDCouncilReportv2.pdf.

11. National Science Foundation, "NSF Announces Future Internet Architecture Awards," August 2010, https://www.nsf.gov/news/news_summ. jsp?cntn_id=117611\&org=OLPA\&from=news.

12. Lixia Zhang, Deborah Estrin, Jeffery Burke, Van Jacobson, Beichuan Zhang, James D. Thornton, Diana K. Smetters, et al. "Named Data Networking (NDN) Project," Technical Report NDN-ooo1, October 31, 2010, https:// named-data.net/publications/techreports/trooindn-proj/.

13. Zhang et al., "NDN Project," 7; J. Takemasa, K. Taniguchi, Y. Koizumi, and T. Hasegawa, "Identifying Highly Popular Content Improves Forwarding Speed of NDN Software Router" (conference paper, 2016 IEEE Globecom Workshops), 1-6, https://doi.org/10.1109/GLOCOMW.2016.7848926.

14. Zhang et al., "NDN Project."

15. Bob Brown, "IP Was Middle School, Named Data Networking Is College," Network World, October 8, 2015, http://www.networkworld.com/article/2990834/network-management/ip-was-middle-school-named-datanetworking-is-college.html; Minerva Bauman, "NMSU Professor Working on New Wireless Networks," Las Cruces Sun-News, September 30, 2017, http:// www.lcsun-news.com/story/news/education/nmsu/2017/o9/30/nmsu-professor-working-new-wireless-networks/718803001/; Rutgers University, "Designing the Future Internet," ScienceDaily, October 20, 2016, https:// www.sciencedaily.com/releases/2016/10/161020115310.htm; and David Talbot, "Your Smartphone and Tablet Are Breaking the Internet," MIT Technology Review, January 9, 2013, https://www.technologyreview.com/s/509721/yourgadgets-are-slowly-breaking-the-internet/.

16. Van Jacobson et al., "Named Data Networking (NDN) Annual 2012-13 Report," 2013, p. 2, http://www.caida.org/publications/papers/2013/named_ data_networking_2012-2013/named_data_networking_2012-2013.pdf.

17. Augusta Ada King Lovelace, Annotated Sketch of the Analytical Engine Invented by Charles Babbage, Extracted from the "Scientific Memoirs," ed. Luigo Federico (R. \& J. E. Taylor, 1843), 710.

18. Van Jacobson, Deborah Estrin, Lixia Zhang, Beichuan Zhang, kc claffy, Dimitri Krioukov, Dan Massey, et al., Named Data Networking (NDN) Annual 2012-13 Report (2013), 10, http://www.caida.org/publications/papers/2013/ named_data_networking_2012-2013/named_data_networking_2012-2013.pdf; in Katie Shilton, "Anticipatory Ethics for a Future Internet: Analyzing Values During the Design of an Internet Infrastructure," Science and Engineering Ethics 21, no. 1 (2015): 9, https://doi.org/10.1007/s11948-013-9510-z.

19. Zhang, personal communication, December 10, 2017.

20. Fisher, "US National Science Foundation and the Future Internet Design." 
21. P. Gusev, personal communication, August 2, 2017.

22. P. Gusev, personal communication, August 2, 2017.

23. J. DeHart, personal communication, November 23, 2018.

24. Stiegler, Technics and Time, 2, 102.

25. For Stiegler's discussion of the complications inherent in "real time," see Technics and Time, 2, 62-63.

26. Stiegler, Technics and Time, 3, 37 .

27. J. Burke, personal communication, September 12, 2016.

28. Galloway, Protocol, 67 .

\section{About the Author}

Britt S. Paris is an Assistant Professor in the Department of Library and Information Science at Rutgers University, New Brunswick. Her work is broadly focused on how values and ethics directives are rolled out in technological projects and information systems and how these activities affect political action. 


\section{Index}

acceleration $9,10,21,26-27,30,32,86,128$, $138,144,177-179,182,245,257,285,291,302$ of culture and life $\quad 9-10,32$ n. 2,36 n. 36 , $42,138,144,177-179,182,257$ of technology $\quad 9^{-10}, 30,128-131,144,285$, 291, 293n, 302

and modernity $\quad 9,19,32 \mathrm{n} .2$

see also speedup

accelerationism 10, 32 n. $2,83,86$

aging (of technology and infrastructures) 14, 31, 62, 66, 191-193, 200-201; see also obsolescence

algorithms $13-14,17,25,28,30,80-82,86$, $89-103,126-142,143-156,157-174$ algorithmic bias 13,33 n. 13, 286 anticipatory shipping $\quad 263-278$ stock trading $28,90,127-129,139$ n. 19, 140 n. 25,182 machine learning $17,82,127,142$ n. 73 , 148-150

recommendation $\quad 80-81,213-216,267,273$ self-driving cars $\quad 157-174$

animation $143^{-156}$

anonymous time $\quad 24-25,90-91$

anticipation $31,80,91,112,158,172$ n. 22, 200, 203 n. 12, 205-219, 263, 266, 270-272 anxiety $31,110,145^{-46}, 15^{2}, 205^{-219}$ artificial intelligence (AI) $83,127,134,139$, 143-156, 279-294

artificial neural networks (ANNs) 143, 146-148, 150, 256, 279-294

Ashby, W. Ross $135^{-136,142}$ n.81, 261 n. 24 atomic clock $95,114-116$

Augustine of Hippo $\quad 41,77-78$

Babbage, Charles $\quad 30,125^{-126,129,134,138} \mathrm{n}$. $4-5,139$ n. 8, 299, 307 n. 17

Barthes, Roland $\quad 63-64,72$ n. 47

being-toward-death $79,83,91$; see also Heidegger, Martin

Benjamin, Ruha 13,33 n. 13

Benjamin, Walter $91,93,101$ n. 6, 258, 262 n. $5^{2}$ Bennett, Jane 12, 33 n. 9

Benveniste, Émile $\quad 20-21,36$ n. 41

Bergson, Henri $\quad 47,78,92,95$

Berlant, Lauren 14,34 n. 22, 217 n. 5, 218 n. 22 big data $17,28,67,80,143-144,208,262,283$ bitcoin 130, 132; see also blockchain

Bloch, Ernst $\quad 101,208,217$ n. 3

blockchain 130,133, 135, 140n, 141n; see also bitcoin and Ethereum

body (human) 10, 31, 44, 46, 49-50, 57, 145, $178-180,183-185,199-200,209,214,296$, 304-05; see also embodied and lived temporalities calendars $11,18-21,36 \mathrm{n}, 111,114,133,228-229$, 269

Canales, Jimena $\quad 25,37$ n. $66,53,172$ n. 14 capitalism $10,12,23-25,28,67,133,137,187$, 226, 234, 276n, 283-286, 303

capitalist time $18-19,23-25$

Carey, James 17,35 n. 35, 180-181, 188 n. 7

cellphone 233-235; see also mobile devices and smartphone

chronic time (Benveniste) 20-21, 36 n. 44; see also Benveniste, Émile

Chun, Wendy 28, 34 n. 16, 69 n. 2, 135, 142 n. 72

clocks $16,18-25,31,37$ n. 61

clocking (microprocessors) 9o-91, 92, 94

quartz clock 94, 226

atomic clock 95,114-116

mechanical clock 21, 90

clock time $19-23,47,96,108,114-115$, 119-120

stopwatch $18,227,229,232$

wristwatch $31,95,102,113,224-225,227$, 234, 238 n. 23 and n. 30, 239 n. 41-44; see also wearables

cloud computing 126,153 n. 6,182

computer time $14,117,128-129,133,137-138$, 143

control (cybernetics) $\quad 67-68,94,208,244,271$

Coordinated Universal Time (UTC) 94-95, 114-117

coordination

of time technologies $\quad 11-15,19-21,28,114$, 116-119, 121-122, 181, 229, 247, 300-301, 304

of social practices $\quad 20-21,23^{-25}, 114,179$, 305

of human and nonhuman actors 27 , 94-95, 114, 296, 305

cosmic time $\quad 20-21$

Crary, Jonathan 33 n. $3,178,188$ n. 5

cultural techniques $15^{-16,35}$ n. 29, 54 n. 17 , $55^{-57}, 68,73$ n. 62,140 n. $29,267,276$ n. 27 cybernetics $14,47,54$ n. 14, 67-68, 94, 135, $147,152,208,218$ n. $8,25^{2-254}, 261$ n. 24 , $271-272,275$ n. 12,277 n. 48

data

tracking and surveillance $210,234,244$, $255^{-258,269-270}$

transmission $17,32,51,94-96,99-100,120$, 144-145, 296-298, 302-303

big data $17,28,67,80,143-144,208,262$, 283

processing $25,28,81,90,92,94,97,99$, $164-165,168,172,254-255,291$

training $144,279,283-285$ 
storage $\quad 28-29,50,92,96,144-145,300$ packets $95^{-97}, 277$ n. $5^{0,296-300,304}$ database $28,147,161,195,203,255,268,270$ data mining 211

death $31,43,46,51,62,79,91,136,167,206$, 210-216, 292

decision-making $29,157,159,162-164,168-170$, 171 n. 8,172 n. $22,270,280$

delay $92,95-96,125,128-129,133,163,181,184$

Deleuze, Gille 34 n. $17,81,87$ n. 8

Derrida, Jacques 80,86 n. 86,292 n. 8 digital

infrastructures $55,64,112,120-121,179$, $184,187,207-208$

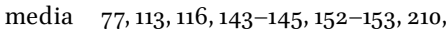
269

networks $14,18,31,111,113,121,153,213$

technology $11,78,82-83,86,107,144-145$, $152,160,211,234$

time $11-12,28,110,112,120-121,124,181$, $232,257,269-270$

diurnal cycle 112-113, 133

Doane, Mary Ann 34 n. 17, 144, 153 n. 1

Edwards, Paul N. $\quad 14,35$ n. 24, 63, 72 n. 45, 252, 260 n. 23

Eigenzeitlichkeit $\quad 68,289$

epoché 98,101

Ernst, Wolfgang $13,29,122$ n. 2, 128, 144, 153 n. 3,154 n. 7 and n. 9,202 n. 3, 244, 259 n. 3 , 272,278 n. 52,292 n. 7

Ethereum 133

Esposito, Elena $\quad 263-264,271-272,277$ n. 45 evolution of technology $78-79,126-127,291$

fiber optics 17,102 n. 13, 181-182, 295

film $13,27,34$ n. $17,44,47-48,154$ n. 13 , $213^{-214}, 228,230,238$ n. $24,247^{-250}, 25^{8}$

Foucault, Michel $\quad 46,49,69$ n. $5,99,120,123$ n. 23,199

future $17,19-20,29-30,32,46,53,57$, $66-67,77-87,135-136,166,206-211,261$ n. $39,265-266,272-273,280-281 ;$ see also prediction and anticipation

Future Internet Design program 297

Galloway, Alexander R. $\quad 116,122$ n. 3,123 n. 27, 277 n. 50, 296, 304, 306 n. 2

gender 56, 239, 257

Global Positioning System (GPS) 95, 161, 181, 210-211

Google $30,117,123$ n. 31, 127-128, 143-156, 208, 211, 256, 259 n. 1, 278 n. $55,280,285,288,295$

Grusin, Richard 33 n. 8208,218 n. 9

Hansen, Mark B. N. 34 n. 16, 144-145, 153 n. 4, 208,218 n. 9,292 n. 7

hardware $28,62,95,108,112,121,187,192-193$, $201,255,284-285,290-291,297$ hardwired temporalities $\quad 11-12,16-18,21,46$, $57-58,79,82,89-90,92,99-100,111,180,187$, 222-223

Hartog, François $\quad 125,135,138,142$ n. 75

Hayles, Katherine 127, 160-61, 171 n. 6, 200

Hegel, Georg Wilhelm Friedrich $45,51,83$

Heidegger, Martin $\quad 25,29,37$ n. 65, 66, 74 n. $65-66,79,83,85,86$ n. 4,87 n. $15,90-92,98$, 101, 209-210, 215, 218 n. 14

Helmholtz, Hermann von 26, 91

high-frequency trading $\quad 127-128,140$ n. 25, 182; see also algorithms, stock trading

history of science and technology $21,56,79$, $82,134,243$

Husserl, Edmund $\quad 29,80,86,93-94,97,101 \mathrm{n}$

information

society and age $90,97,244$

theory $162-163,252$

processing $97,162-164,172$ n. 15, 254, 290-291; see also data processing

infrastructure

aging $14,31,62,66,191-192,193,200-201$; see also obsolescence

as material $9,14,18,19$

as social form 14

and modernity $14,19,23,101,181$

care of $55^{-58}$

digital $55,64,112,120-121,179,184,187$, 207-208

network 145,179

temporality of $14,55^{-58,60-61}$

of temporality $9-12,15,18,21,23,25,27-28$ repair $14,29,35 \mathrm{n}, 56-57,60-61,64,67-68$, $179,184-185,187$

maintenance $10,29,36,55^{-57}, 59-60$, $65^{-68,177,179,184-185,194,200,202}$

Innis, Harold 20,36 n. 40, 45

innovation $13,19,35$ n. 25,142 n. $79,200,202$, 224, 266, 279, 288, 300

instantaneity $10,43,91,93,127,160,178$ integrated circuit $\quad 15,17-18,35$ n. 34,36 n. 37 , 93-94, 99-100, 126, 131, 222-223, 226-228, $231,235,244$ central processing unit (CPU) 164, 304 graphics processing unit (GPU) 163, 290 as semiconductors 99, 222, 228, 236 n. 9. 237 n. 17,238 n. 26

interface (temporal) $19,27,187$

interface (computer) 108, 112, 122 n. $3,143,146$, $149-153,155$ n. $28,162-163,168,182,187,195$, $245,256-258,302-305$

internet $\quad 28-29,33,90,92,99,109-110,113$, $116-117,133,135,136,179,182,186,209$, 211-212, 245, 257, 295-305

Internet time $\quad 95^{-} 97,109,117,124$ n. 33 iPhone 61, 234; see also smartphone and mobile devices

irreversibility $21,29,5^{0}, 5^{2}, 217$ 
Jackson, Steven 35 n. 25, 57, 61, 64, 69 n. 1, $202 \mathrm{n} .8$

Kittler, Friedrich $\quad 29,32,34$ n. 14, 41, 48-49, 51, 54,123 n. $16,129,132,139$ n. 9,140 n. 33,141 n. 53, 192, 202 n. 2, 290-291, 294 n. 29-32

Krämer, Sybille 36 n. 39, 50, 54 n. 17, 129, 134, 140 n. 29

labor $15,19,21,29,56,166,177-182,185,187$, $226,232,237,256-257,269,281,283$

labor-time $15,19,21,23-25,35$ n. 27, 232, 235

latency $178,182,188 \mathrm{n} .11-13,201,285,303,305$

Latour, Bruno $\quad 15,33$ n. 8,35 n. 26 and 28,56 , 64,70 n. $10-11,71$ n. 19 and $23,145,154$ n. 7 , 265,275 n. 9,292 n. 5

leap second $\quad 29-30,102,107-109,114-122,128$ legacy systems 191-203; see also obsolescence Leibniz, Gottfried Wilhelm $\quad 78,83$

leisure time 12,232

linear time $\quad 5^{0}, 131-132$

logistical media $\quad 24,263,264,267-270,276 \mathrm{n}$. 27 and 33,277 n. 40

logistics $11,29,33$ n. $7,68,263-265,267-269$, $275,276 \mathrm{n} .25$ and 37

longue durée 9,35 n. 30, 138

Marx, Karl $\quad 15,35$ n. 27, 235

Massumi, Brian $\quad 208,218$ n. 9

McLuhan, Marshall 9o, 96-98, 102 n. 22

McIlwain, Charlton 13,33 n. 12

media

archaeology of 13,34 n. 15, 89-90, 92, 97, $100,103,154$ n. 7 and $13,161,274$ n. 4,282 culture $10,94,100$ digital $77,113,116,143^{-145}, 15^{2-153}, 210$, 269

definition of

logistical $24,263,264,267-270,276$ n. 27 and 33,277 n. 40

mass 258

as time manipulation $9,34 \mathrm{n} .14,47-48$, $50-51,54$ n. 17,129

theory of $9,45,48,51,96,124,138,153$ n. 2 , 263,277 n. 41

mass 258

technology $\quad 9,13-14,50,69$ n. 1, 92, 289 time-critical (zeitkritische Medien) 13, 28-29, 34 n. 16, 41, 95-97, 99-100, 102, 108-109, 116, 128, 161-162, 167, 244 memory (computer) 28, 34 n. 16, 96, 131-132,

135,139 n. 24, 142 n. $74,272,288,300,302$ memory (human and social) $36 \mathrm{n} .5^{0}, 5^{2}, 80$,

$84,90-91,96,102$ n. $16,201,253,261$ n. 26 , 299

microprocessor see integrated circuit microchip see integrated circuit microtime $9,11-12,25-30,18-19,25-30,94$, 99; see also microtemporality microtemporality $9,11-12,18-19,25-30,94$ 99-100, 108, 128-129, 142 n. 74, 146, 149, 157 , 159, 161-162, 167-168, 170, 180, 223, 228, 232, $235,280,292 \mathrm{n} .7$ see also microtime mobile devices $64,69 \mathrm{n} .2,98,103,221$, 223, 233-235, 239 n. 47, 256-257, 262 n. 45, 269-270, 297; see also cellphone and smartphone

modern time 90

Mumford, Lewis $\quad 21-23,36$ n. 46, 304

Named Data Networking (NDN) 32, 295-308 neoliberal economy $12,111,120,205,286$ network

cable 17, 30, 6o, 92, 99, 177-189, 209

digital 14, 18, 31, 111, 118, 121, 153, 213

telegraph $\quad 17,35$ n. $35,92-93,178,180-183$

telephone $99,127,183$

undersea $\quad 177^{-189}$

time 112,140 n. 36,186

wireless 98,205

Network Time Protocol (NTP) 95, 116-117, 137

new materialism $\quad 2,12,33 \mathrm{n} .9$

Newton, Isaac $\quad 47-48,132$

Noble, Safiya Umoja 13,33 n. 11

nonhuman $12,15,19,27-28,33$ n. $8-9,56$, 89-9o, 96, 107-109, 111, 116, 120, 146, 149, $151-153,180,187$

obsolescence $15,17,31,59-62,67,69$ n. 2,187 , 191, 193, 198-201, 203 n. 14; see also legacy systems

pendulum 91, 93, 247

Peters, John Durham 16, 20, 22, 24, 29, 34 n. 21, 37 n. $61,200,203,263,268-269,276-277$ phenomenology $29,80,86,97,101$ n. 7, 205 practice turn 13,34 n. 20,155 n. 30 preemption $31,77,81,86,159,164,207-208$, 212, 217 n. 6

prediction $16-17,20,29-30,32,80,94,134$, $136,145,158,164-166,170-171,206,208$, 211-212, 233, 249-253, 263-267, 270-273, $280,285^{-286}$

predictive policing 13,208

processing see data processing

prophecy $263,270-271$

protocol 11, 18, 123 n. 27 and 29, 137, 140 n. 36 , 166, 169, 295-296, 301, 303-305; see also Transmission Control Protocol/Internet Protocol, Network Time Protocol, and Named Data Networking

radio $27,91-93,95,98-100,118,205,270$

real-time $10,13,28,33$ ก. $10,50-52,91,94,97$, 113,123 n. $16,129,133,138,140$ n. 30, 160, 178 , 256, 263, 269, 284, 293 n. 14, 298, 300-304, 308 n. 25

repair studies $\quad 56$ 
reversibility $23,29,47-48,50,5^{2}$

rhythm

bodily $\quad 31,177-189$

circadian 20-21, 27, 130; also as cosmic and celestial

seasonal 20,36 n. 50, 133

environmental $16,24,31$

technological 14, 23, 57, 94, 98-99, $102 \mathrm{n}$. 24,103 n. $25,149,272$

work 24, 57, 202 n. 8, 252

Ricoeur, Paul 20, 24, 34 n. 17,36 n. 42

routine $55-57,60,168,183,185,191,213,233$

Schabacher, Gabriele $\quad 29,31,69$ n. 1, 71 n.19, 72 n. 36,73 n. $5^{1}$ and 57,74 n. 67,102 n. 17 , 179,188 n. $6,267-268,276$ n. 25,277 n. 5 o, 292 n. 9

science and technology studies $11-12,15,56$, 169, 281-282

semiconductors $99,222,228,236$ n. 9. 237 n. 17,238 n. 26; see also integrated circuits

Serres, Michel $15^{-16}, 35$ n. 28, 280, 292 n. 5

Shannon, Claude $\quad 99,103$ n. 31, 163

Sharma, Sarah $\quad 10,33$ n. 5, 178-179, 183, 257

Simondon, Gilbert 114,123 n. 19 and 22, 146 smartphone 10, 18, 31, 185, 205-206, 209-210, 221-223, 233-235, 245, 256-257, 297; see also cellphone and mobile devices

slowness $10,49,177-180,184,186$ social media 33 n. 10, 80, 87 n. 11, 90, 102,107, 123 n. 16, 127, 205, 207, 213, 257, 288, 297 software

and adaptability $\quad 16$

as applications $147,15^{1}, 159,210,255^{-256}$, 291

and bias $13,33 n$

care for $3^{0-31,56,62,64,69 ~ n . ~ 2, ~ 97, ~}$ 191-203

development $\quad 111-113,147,274$

and hardware $31,62,95,108,121,191-203$

materiality of $31,191-203$

and time 123 n. 29, 133 191-203

software studies $192,203 \mathrm{n} .12$

speed

concept of 303,305

as efficiency $303-304$

of light $91,98,127,130,303$

beyond perception

processing 17, 26, 121, 131, 163, 168, 172 n. 15 , 285

theory of 178,188 n. 5

speedup $9,12,26,28,32,245$; see also acceleration

speed runs 150-151

Star, Susan Leigh $\quad 13,27,34$ n. 22, 37 n. 60 and 72,70 n. $7,122,293$ n. 10

Stiegler, Bernard $\quad 29,32,77,80,86$ n. 6, 258, 262, 295-296, 299, 301-302, 304, 306 n. 4-5, 308 n. 25 streaming (video) 10, 50, 96, 297-298, 300, 303-304; see also video

synchronization $9,13-15,18,23,35,82,84$, 89-90, 92-95, 97, 99, 111-113, 116-117, 123, $126,129,133,136,140$ n. $36,144,161,164,233$, $247,250,25^{8}$

techné 85,100

technics 36 n. $46,85,86$ n. 6,87 n. $14,144,153$, $180,207,210,258,262,295^{-296}, 302,306 \mathrm{n}$. 4-5

technological determinism 86,100 technological singularity $82-83,135,139,141$ n. $62,280-281,291,292$ n. 4

telecommunications $\quad 95,117-118,177-178$

television $10,27,95,129,183$

temporal regimes $11-12,16-28,57-60,63$, $69-70,89,95,107,109-122,128,181,184,187$, 191, 196, 199-200, 203, 284, 289 colonial $133,137-138,177,181,183-184,187$ capitalist $10,12,18-19,23-25,28,33$ n. 3 , 36 n. 36,37 n. $56,133,137,187,226,234$, 238 n. 35, 284-286; see also capitalism and clocks: clock time

calendar 18, 20-21; see also calendar monastic 21, 90

temporality

etymology of 16

hardwired 11-12, 16-18, 21, 46, 57-58, $79,82,89-90,92,99-100,111,180,187$, 222-223

soft $31,100,177,180,187$

embodied or lived 70 n. 14, 162, 179-180, $182-183,187$

Thompson, E. P. $\quad 24,37$ n. $56,133,144,238$ n. 35 time

anonymous $\quad 24-25,90-91$

capitalist $\quad 18-19,23-25$

chronic (Benveniste) 20-21, 36 n. 44; see also Benveniste, Émile

clock 19-23, 47, 96, 108, 114-115, 119-120

computer $14,117,128-129,133,137-138,143$

Coordinated Universal Time (UTC) 94$95,114-117$

cosmic 20-21

digital 11-12, 28, 110, 112, 120-121, 124, 181, $232,257,270$

internet $95^{-97,109,117,124}$ n. 33

historical $16,77,82,132-133,138$

human perception of $26-27,29-30$, $42-45,48,50,53$ n. $2,92,95-97,108$, $112,118,127-130,132,137,144-146,162$, 250, 304

labor-time $15,19,21,23-25,35$ n. 27, 232, 235

leisure 12,232

linear 50, 131-132

measurement of $19-20,30,36 \mathrm{n} .48,108$, $112-120$ 
media 20,25

micro 9, 11-12, 18-19, 25-30, 94, 99-100, 128,142 n. $74,146,149,157,159,161-162$, $167-168,170,180,223,292$ n. 7

modern 90

network 112,140 n. 36,186

nonhuman $15,27,89-90,96,187$

order of $116,118-121$

reaction $15,26,158,162-164,168,172$ n. 14 and 16

real-time $10,13,28,33$ n. $10,5^{0}-5^{2}, 91,94$, $97,113,123$ n. $16,129,133,138,140$ n. 30 , $160,178,256,263,269,284,293$ n. 14, 298, $300-304,308$ n. 25

religious 12

standardization of $11,19,23,36 \mathrm{n} .5^{0}, 56$, $114,120,181-182,187,224,284$; see also Coordinated Universal Time

time servers $95,124 \mathrm{n} .33$

time zones $\quad 95,109,111-112$

universal $24,94,113-114,116,120,187$

time-axis manipulation 34 n. $14,47-48$, $5^{0-51,54}$ n. 17,129 time-criticality (Das Zeitkritische) 13, 28-29, 34 n. 16, 41, 95-97, 99-100, 102, 108-109, 116, $128,161-162,167,244$

timekeeping $18,20,22-23,93,95,114,223$, $225^{-227,231,233}$

Transmission Control Protocol/Internet Protocol (TCP/IP) 32, 97, 296-298, 300

video games $143-44,150,178,229,231,234$

Virilio, Paul 32 n. 2, 144, 178, 188 n. 5, 291, 294 n. 33

von Baer, Karl Ernst $\quad 26,37$ n. 69, 41-44, $46-47,49$

von Neumann, John $\quad 46,290$

waiting $10,58,66-69,73-74,80,178,184-185$, 244,253

wearable 209, 234, 239 n. 43; see also clock: wristwatch

Whitehead, Alfred North $119-120,124 \mathrm{n}$. $34-35,174$ n. 37

Wiener, Norbert $\quad 46-48,54$ n. 14, 100, 208, 218 n. $8,253,261$ n. 24 and 29,271 



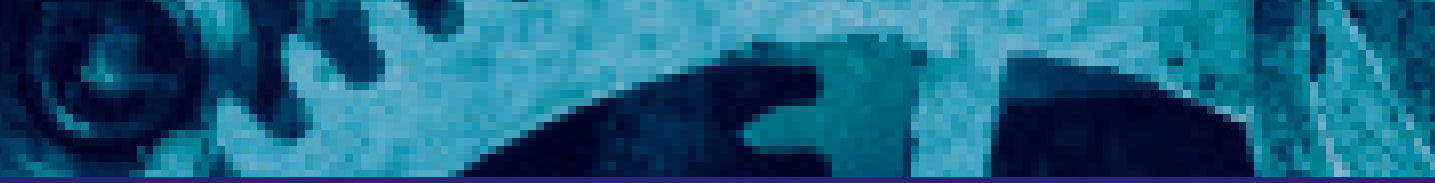

In a crucial sense, all machines are time machines. The essays in Media Infrastructures and the Politics of Digital Time develop the central concept of hardwired temporalities to consider how technical networks and infrastructural practices hardwire and rewire patterns of time. Digital media introduce new temporal patterns in their features of instant communication, synchronous collaboration, intricate time management, and continually improued speed. They construct temporal infrastructures that affect the rhythms of lived experience and shape social relations and practices of cooperation. Interdisciplinary in method and international in scope, the volume draws together insights from media and communication studies, cultural studies, and science and technology studies while staging an important encounter between two distinct approaches to the temporal patterning of media infrastructures, a north American strain emphasizing the social and cultural experiences of lived time and a European tradition, prominent especially in Germany, focusing on technological time and time-critical processes.

AKEL UOLMAR is a Postdoctoral Fellow at the Collaborative Research Center "Media of Cooperation" at the University of Siegen. He is co-editor of Format Matters: Standards, Practices, and Politics in Media Cultures and Interrogating Datafication: Towards a Prakeology of Data.

KYLE STInE teaches Film and Media Studies at Johns Hopkins University. His writings on cinema and technology have appeared in Critical Inquiry, Discourse, Grey Room, and the Journal of Cinema and Media Studies.

AUP.nl

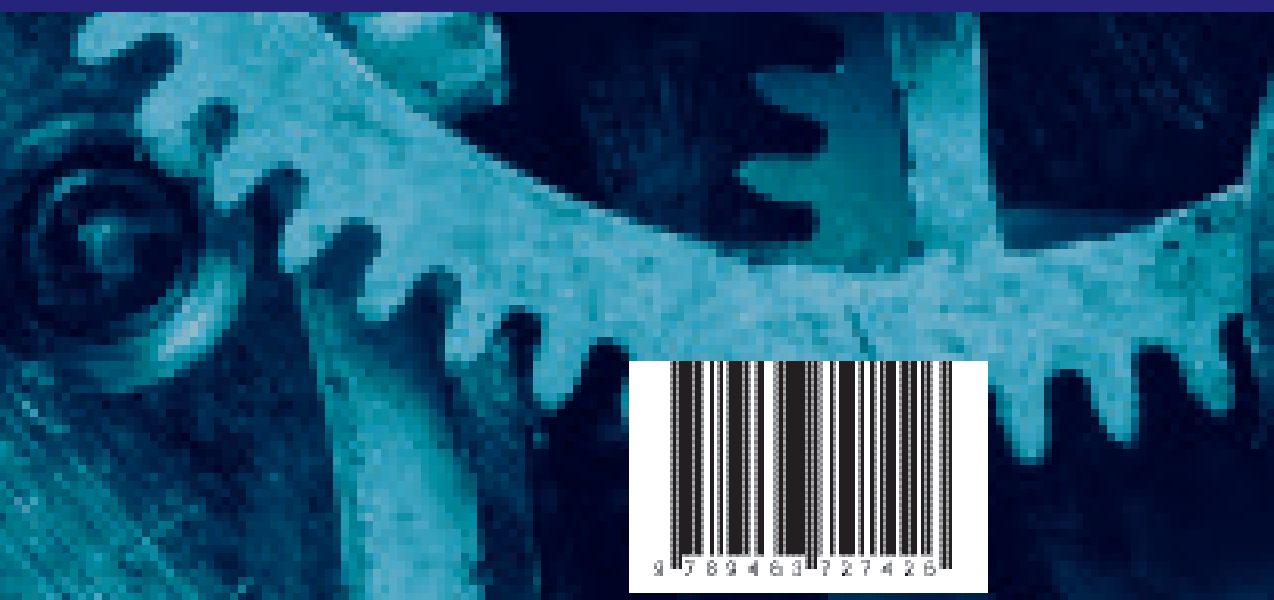

